Prepared in cooperation with the U.S. Army Corps of Engineers

\title{
Annual Exceedance Probabilities and Trends for Peak Streamflows and Annual Runoff Volumes for the Central United States During the 2011 Floods
}

Chapter D of 2011 Floods of the Central United States

2011 Fods of the Centalunited Stas

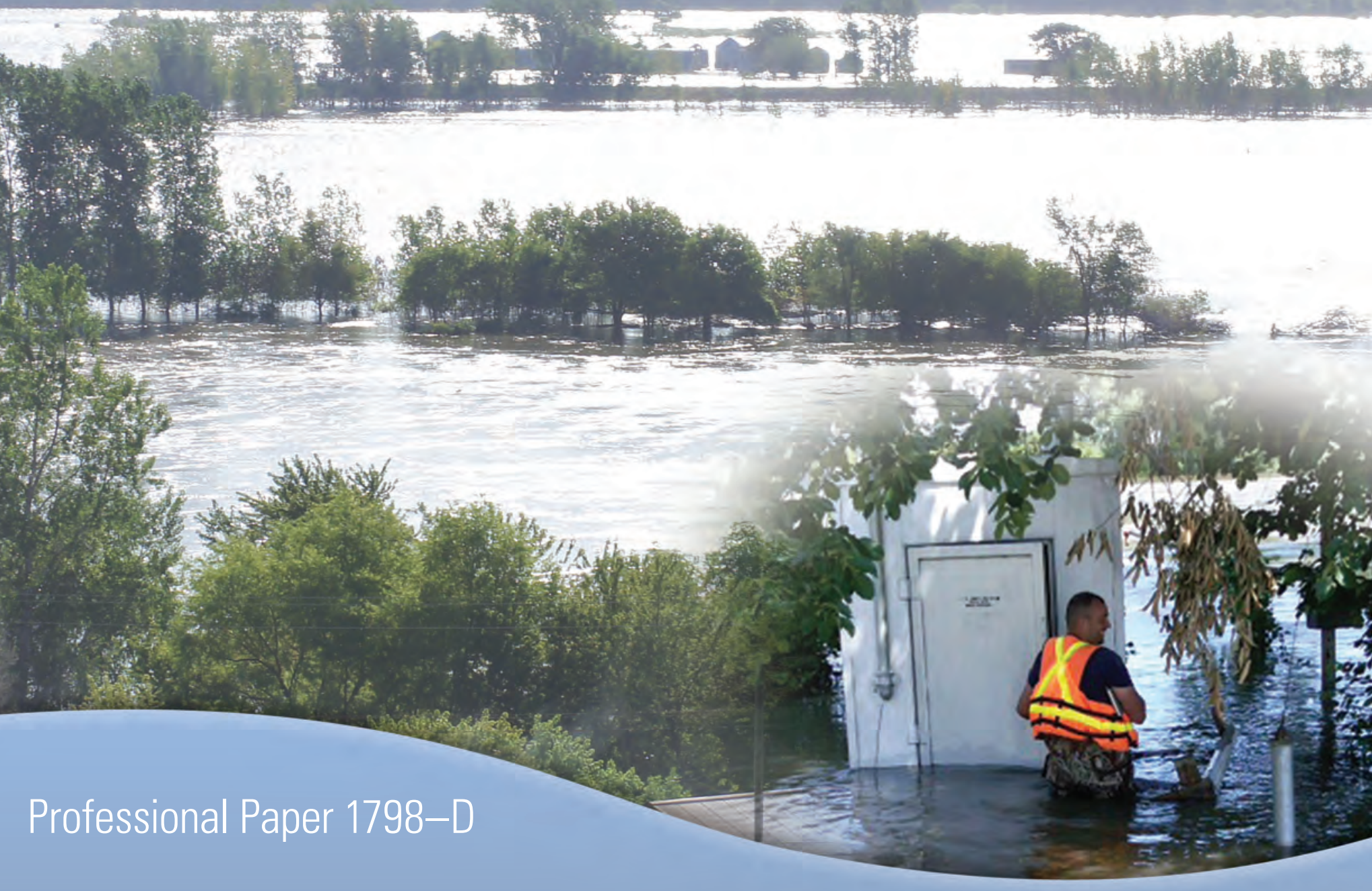


Front cover. Top photograph: View of flooding from Nebraska City, Nebraska, looking east across the Missouri River, August 2, 2011. Photograph by Robert Swanson, USGS.

Right photograph: Flooding along the Missouri River at Blair, Nebraska, June 28, 2011. Photograph by Kevin Richards, USGS.

Back cover. Looking upstream to spillway at Garrison Dam, June 11, 2011. Photograph by Nathan A. Stroh, USGS. 


\section{Annual Exceedance Probabilities and Trends for Peak Streamflows and Annual Runoff Volumes for the Central United States During the 2011 Floods}

By Daniel G. Driscoll, Rodney E. Southard, Todd A. Koenig, David A. Bender, and Robert R. Holmes, Jr.

Prepared in cooperation with the U.S. Army Corps of Engineers

Professional Paper 1798-D 


\title{
U.S. Department of the Interior SALLY JEWELL, Secretary
}

\section{U.S. Geological Survey Suzette M. Kimball, Acting Director}

\author{
U.S. Geological Survey, Reston, Virginia: 2014
}

For more information on the USGS - the Federal source for science about the Earth, its natural and living resources, natural hazards, and the environment, visit http://www.usgs.gov or call 1-888-ASK-USGS.

For an overview of USGS information products, including maps, imagery, and publications, visit http://www.usgs.gov/pubprod

To order this and other USGS information products, visit http://store.usgs.gov

Any use of trade, firm, or product names is for descriptive purposes only and does not imply endorsement by the U.S. Government.

Although this information product, for the most part, is in the public domain, it also may contain copyrighted materials as noted in the text. Permission to reproduce copyrighted items must be secured from the copyright owner.

Suggested citation:

Driscoll, D.G., Southard, R.E., Koenig, T.A., Bender, D.A., and Holmes, R. R. Jr., 2014, Annual exceedance probabilities and trends for peak streamflows and annual runoff volumes for the Central United States during the 2011 floods, U.S. Geological Survey Professional Paper 1798-D, 89 p., http://dx.doi.org/10.3133/pp1798D. 


\section{Contents}

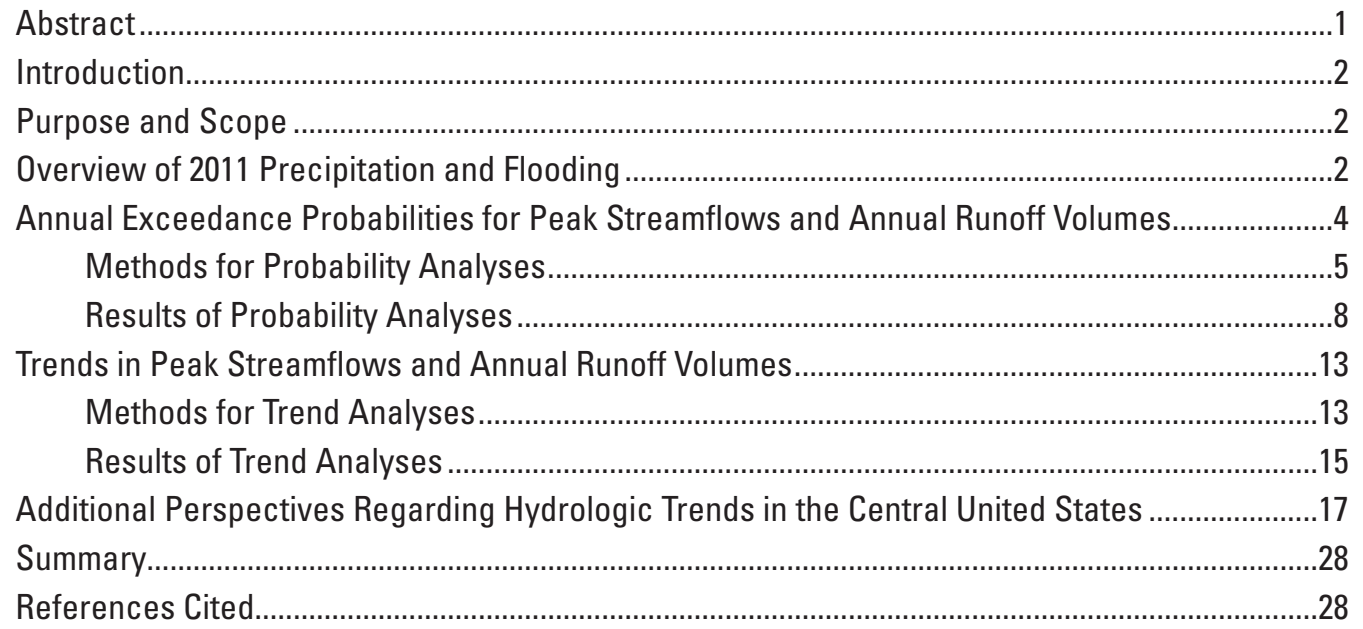

\section{Figures}

1. Map showing major river basins of the Central United States along with other locations affected by flooding streams from February to September, 2011

2. Map showing locations of selected streamgages in the Central United States where flooding took place during February to September, 2011.

3. Map showing annual exceedance probabilities for 2011 peak streamflows for selected streamgages in the Central United States affected by 2011 flooding

4. Map showing annual exceedance probabilities for 2011 annual runoff volumes for selected streamgages in the Central United States affected by 2011 flooding ...

5. Map showing percentage changes in median annual peak streamflow values (1958 to 2011) for selected streamgages in the Central United States affected by 2011 flooding

6. Map showing percentage changes in median annual peak streamflow values (1958 to 2007) for selected streamgages in the Central United States previously reported by Holmes and others (2010)

7. Map showing percentage changes in median annual runoff volumes (1958 to 2011) for selected streamgages in the Central United States affected by 2011 flooding .........19

8. Graph showing time-series (1929 to 2011) record of annual peak streamflow for the James River near Scotland, South Dakota...

9. Graph showing time-series (1929 to 2011) record of annual runoff volume for the James River near Scotland, South Dakota.....

10. Graph showing lake-level record for Waubay Lake within the Waubay Lakes Chain in northeastern South Dakota.

11. Graph showing lake-level record for Devils Lake in northeastern North Dakota ...........23

12. Graphs showing sum of normalized ranks for peak streamflows, by water year (1958 to 2011).

13. Graphs showing sum of normalized ranks for annual runoff volume, by water year (1958 to 2011).... 


\section{Tables}

1. Peak streamflow data and results of flood-probability analyses for selected streamgages in the Central United States affected by 2011 flooding...

2. Annual runoff data and results of flood-probability analyses for selected streamgages in the Central United States affected by 2011 flooding

3. Summary of results of probability analyses for selected streamgages in the Central United States for annual peak streamflows (from table 1) and annual runoff volumes (from table 2).

4. Results of trend analyses (1958 to 2011) for selected streamgages in the Central United States affected by 2011 flooding.

\section{Conversion Factors}

Inch/Pound to SI

\begin{tabular}{|c|c|c|}
\hline Multiply & By & To obtain \\
\hline \multicolumn{3}{|c|}{ Length } \\
\hline inch (in.) & 2.54 & centimeter $(\mathrm{cm})$ \\
\hline inch (in.) & 25.4 & millimeter (mm) \\
\hline foot $(\mathrm{ft})$ & 0.3048 & meter $(\mathrm{m})$ \\
\hline mile (mi) & 1.609 & kilometer (km) \\
\hline \multicolumn{3}{|c|}{ Area } \\
\hline acre & 4,047 & square meter $\left(\mathrm{m}^{2}\right)$ \\
\hline acre & 0.4047 & hectare (ha) \\
\hline acre & 0.4047 & square hectometer $\left(\mathrm{hm}^{2}\right)$ \\
\hline acre & 0.004047 & square kilometer $\left(\mathrm{km}^{2}\right)$ \\
\hline square mile $\left(\mathrm{mi}^{2}\right)$ & 259.0 & hectare (ha) \\
\hline square mile $\left(\mathrm{mi}^{2}\right)$ & 2.590 & square kilometer $\left(\mathrm{km}^{2}\right)$ \\
\hline \multicolumn{3}{|c|}{ Volume } \\
\hline acre-foot (acre-ft) & 1,233 & cubic meter $\left(\mathrm{m}^{3}\right)$ \\
\hline acre-foot (acre-ft) & 0.001233 & cubic hectometer $\left(\mathrm{hm}^{3}\right)$ \\
\hline \multicolumn{3}{|c|}{ Flow rate } \\
\hline cubic foot per second $\left(\mathrm{ft}^{3} / \mathrm{s}\right)$ & 0.02832 & cubic meter per second $\left(\mathrm{m}^{3} / \mathrm{s}\right)$ \\
\hline
\end{tabular}

Vertical coordinate information is referenced to the North American Vertical Datum of 1988 (NAVD 88).

Horizontal coordinate information is referenced to the North American Datum of 1983 (NAD 83).

Water year is the 12-month period from 0ctober 1 to September 30 and is designated by the calendar year in which it ends. For example, water year 2011 is from October 1, 2010 to September 30, 2011. 


\title{
Annual Exceedance Probabilities and Trends for Peak Streamflows and Annual Runoff Volumes for the Central United States During the 2011 Floods
}

\author{
By Daniel G. Driscoll, Rodney E. Southard, Todd A. Koenig, David A. Bender, and Robert R. Holmes, Jr.
}

\begin{abstract}
During 2011, excess precipitation resulted in widespread flooding in the Central United States with 33 fatalities and approximately $\$ 4.2$ billion in damages reported in the Red River of the North, Souris, and Mississippi River Basins. At different times from late February 2011 through September 2011, various rivers in these basins had major flooding, with some locations having multiple rounds of flooding. This report provides broadscale characterizations of annual exceedance probabilities and trends for peak streamflows and annual runoff volumes for selected streamgages in the Central United States in areas affected by 2011 flooding.

Annual exceedance probabilities (AEPs) were analyzed for 321 streamgages for annual peak streamflow and for 211 streamgages for annual runoff volume. Some of the most exceptional flooding was for the Souris River Basin, where of 11 streamgages considered for AEP analysis of peak streamflow, flood peaks in 2011 exceeded the next largest peak of record by at least double for 6 of the longest-term streamgages (75 to 108 years of peak-flow record). AEPs for these six streamgages were less than 1 percent. AEPs for 2011 runoff
\end{abstract}

volumes were less than 1 percent for all seven Souris River streamgages considered for AEP analysis. Magnitudes of 2011 runoff volumes exceeded previous maxima by double or more for 5 of the 7 streamgages (record lengths 52 to 108 years).

For the Red River of the North Basin, AEPs for 2011 runoff volumes were exceptional, with two streamgages having AEPs less than 0.2 percent, five streamgages in the range of 0.2 to 1 percent, and four streamgages in the range of 1 to 2 percent. Magnitudes of 2011 runoff volumes also were exceptional, with all 11 of the aforementioned streamgages eclipsing previous long-term (62 to 110 years) annual maxima by about one-third or more.

AEPs for peak streamflows in the upper Mississippi River Basin were not exceptional, with no AEPs less than 1 percent. AEPs for annual runoff volumes indicated less frequent recurrence, with 11 streamgages having AEPs of less than 1 percent. The 2011 runoff volume for streamgage 05331000 (at Saint Paul, Minnesota) exceeded the previous record (112 years of record) by about 24 percent.

An especially newsworthy feature was prolonged flooding along the main stem of the Missouri River downstream from Garrison Dam (located upstream from Bismarck, North

Flood inundation along the Souris River in Minot, North Dakota, June 27, 2011. Photograph by Brent Hanson, USGS. 
Dakota) and extending downstream throughout the length of the Missouri River. The 2011 runoff volume for streamgage 06342500 (at Bismarck) exceeded the previous (1975) maximum by about 50 percent, with an associated AEP in the range of 0.2 to 1 percent.

In the Ohio River Basin, peak-streamflow AEPs were less than 2 percent for only four streamgages. Runoff-volume AEPs were less than 2 percent for only three streamgages. Along the lower Mississippi River, the largest streamflow peak in 91 years was recorded for streamgage 07289000 (at Vicksburg, Mississippi), with an associated AEP of 0.8 percent.

Trends in peak streamflow were analyzed for 98 streamgages, with 67 streamgages having upward trends, 31 with downward trends, and zero with no trend. Trends in annual runoff volume were analyzed for 182 streamgages, with 145 streamgages having upward trends, 36 with downward trends, and 1 with no trend. The trend analyses used descriptive methods that did not include measures of statistical significance. A dichotomous spatial distribution in trends was apparent for both peak streamflow and annual runoff volume, with a small number of streamgages in the northwestern part of the study area having downward trends and most streamgages in the eastern part of the study area having upward trends.

\section{Introduction}

Major flooding at various times during 2011 caused 33 fatalities and approximately $\$ 4.2$ billion in damages in areas spanning from the Canadian border to the Gulf of Mexico and the Rocky Mountains to the Allegheny Mountains (National Oceanic and Atmospheric Administration, 2011). Affected river basins included parts of the Red River of the North (frequently referred to simply as Red River throughout this report), the Souris River, upper Mississippi River, Missouri River, Ohio River, and middle and lower Mississippi River, in the Central United States (fig. 1) and the southern Prairie Provinces of Canada. The flooding began in earnest in February and March in the lower Missouri River, middle and lower Mississippi River, and Ohio River Basins. Snowmelt flooding began during late March and early April in the Red River and upper Mississippi River Basins, followed by successive flooding in the lower Mississippi River, and Missouri River Basins (second round) from mid-April through August (Holmes and others, 2013). The Souris River, which is a major tributary to the Red River, experienced a second round of flooding in June 2011. The 2011 floods in the Central United States were caused by one or more combinations of the following: saturated antecedent soil conditions, higher than average streamflow at the beginning of 2011, rapid melting of the larger than normal snowpack, excess precipitation on snowpack, or simply excess precipitation (Holmes and others, 2013). In some parts of the Central United States, precipitation amounts in excess of 20 inches in a 2-week period (700- to 1,000-percent above normal) were observed (National Oceanic and Atmospheric Administration, 2012a) following snowpack greater than 150 percent of normal in parts of the northern U.S. Rocky Mountains (National Oceanic and Atmospheric Administration, 2012b). During the 2011 flooding, the U. S. Geological Survey (USGS) made more than 2,300 measurements of streamflow at USGS streamgages and other strategic river locations to document peak streamflows, stages, and runoff volumes at locations throughout the Central United States (Holmes and others, 2013).

\section{Purpose and Scope}

In 2011, the USGS operated a network of more than 7,800 streamgages throughout the United States, providing data that are vital for human, economic, and environmental welfare. As the principal federal agency charged with documenting water resources of the United States and assessing natural hazards, it is important to document floods, particularly those of exceptional proportions. Streamflow data from USGS (U. S. Geological Survey, 2013) and other agencies, with some data existing back to the late 1800 s, are important to place context to magnitudes of the 2011 floods. This report provides broadscale characterizations of annual exceedance probabilities and trends for peak streamflows and annual runoff volumes (frequently referred to simply as runoff volume throughout this report) for selected streamgages in the Central United States in areas affected by 2011 flooding. Streamgages considered are the same as those considered by Holmes and others (2013), who documented peak streamflows, stages, and runoff volumes for the 2011 floods.

\section{Overview of 2011 Precipitation and Flooding}

Vining and others (2013) documented the general weather conditions and precipitation contributing to the 2011 flooding in the Central United States. Severe flooding in the Mississippi River and Red River Basins during spring and summer 2011 resulted from weather conditions during December 2010 through July 2011 that were affected, in part, by a La Niña climate pattern. This pattern is characterized by cooler than average sea surface temperatures in the central and east-central equatorial Pacific Ocean and a strong high pressure area in the northern Pacific Ocean. This pattern tends to produce cooler and wetter than normal weather conditions over the western and north-central regions of the Mississippi River and Red River Basins, and often warmer and drier than normal weather conditions over the southern Mississippi River Basin. 


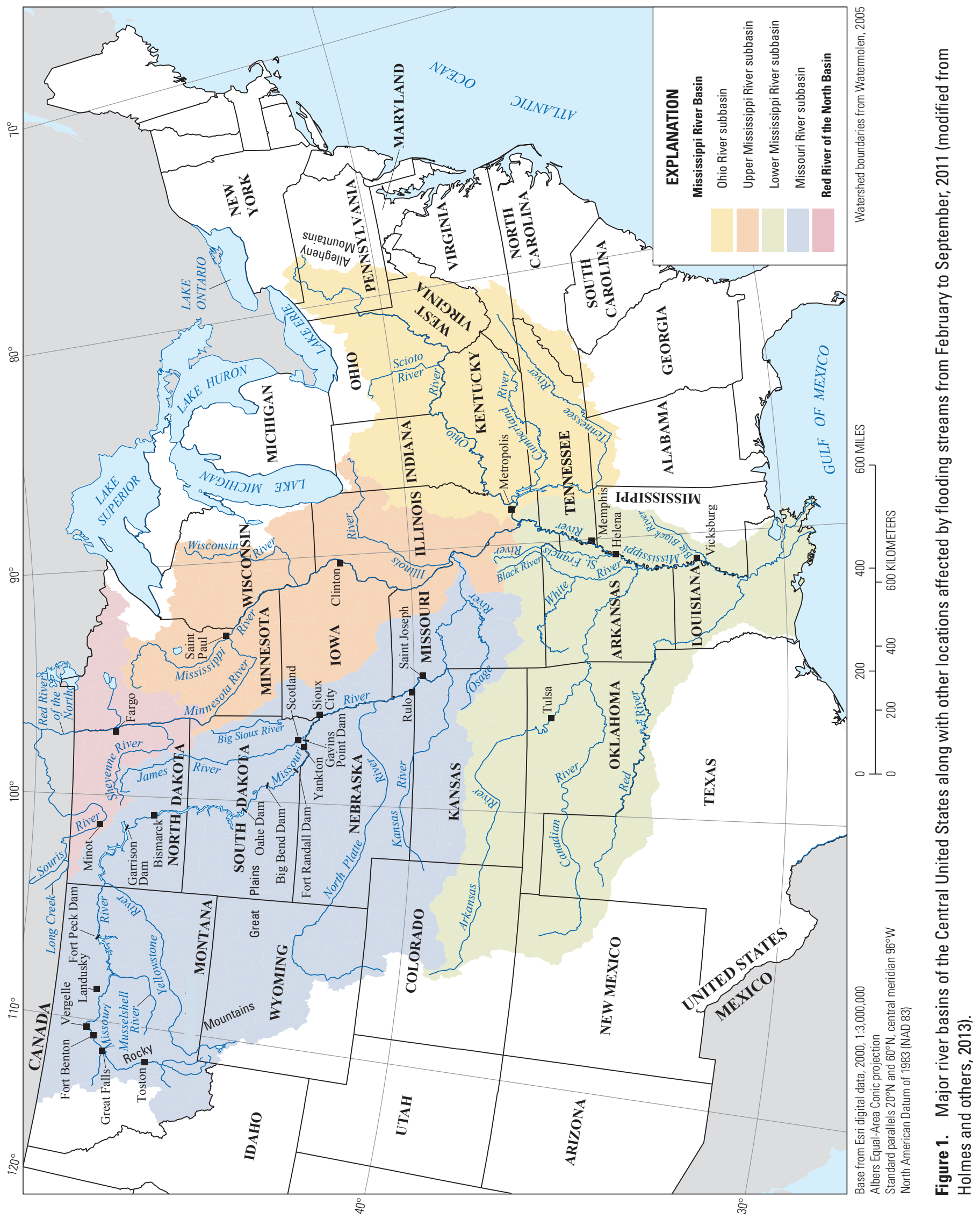


Precipitation patterns across the Red River and Mississippi River Basins from December 2010 through July 2011 followed the mean position of the jet stream (Vining and others, 2013). During December, January, and February, a southward push of the jet stream steered several low-pressure troughs across the country from the Rocky Mountains into the Ohio River Basin producing copious precipitation. From March through May, a large low-pressure trough dominated the continental States, and a high-pressure ridge was centered near the Gulf of Mexico. This weather combination produced storms with abundant precipitation along frontal boundaries that were steered across the Central United States. During June and July, moisture from the southwestern monsoon and the Gulf of Mexico worked into the Plains States. Low-pressure troughs moving across the country interacted with the moist unstable air; thunderstorm development along frontal boundaries resulted in areas of heavy rain across the Northern Plains States into the Missouri and upper Mississippi River Basins. Excess precipitation fell on already saturated ground and ran off into already full streams and rivers, producing severe flooding in the Mississippi River and Red River Basins during spring and summer 2011.

Holmes and others (2013) documented peak streamflows, stages, and runoff volumes for the 2011 flooding in the Central United States. Flooding began in late February 2011 in the Mississippi River Basin and continued in parts of the Mississippi River or Red/Souris River Basins until September 2011. Some locations received multiple flood pulses; the Souris River received two separate flood pulses, and the lower Mississippi River had three separate flood pulses. Peak streamflow records were broken at 105 streamgages considered by Holmes and others (2013) across the Souris/Red and
Mississippi River Basins, with many previous peaks being longstanding records. For example, the 2011 peak streamflow on the Souris River above Minot, North Dakota broke a record set more than 100 years prior. The widespread major flooding in the upper Mississippi River, Missouri, and Ohio Basins in 2011 resulted in record peak streamflows in the lower Mississippi River; in some locations breaking records established in 1927. Records for annual runoff volume were established at 47 streamgages considered by Holmes and others (2013) in the Souris/Red and Mississippi River Basins, with no annual runoff records occurring in the Ohio or lower Mississippi River Basins.

\section{Annual Exceedance Probabilities for Peak Streamflows and Annual Runoff Volumes}

Holmes and others (2013) provided rankings of 2011 peak streamflows or 2011 annual runoff volumes for 497 streamgages (fig. 2) in the Central United States for comparison with previous large floods. Although rankings help to illustrate relative magnitudes of the floods, evaluation of future flood risk requires a probability analysis that involves determining parameters needed to estimate a probability distribution from a set of observed streamflow data. The probability distribution relates probability to the magnitude of a certain size flood peak (or annual runoff volume) being equaled or exceeded, from which annual exceedance probabilities (AEPs) can be estimated.

\section{Flooding along the Missouri River at Sioux City, lowa, at the I-29/ Hamilton Street interchange, June 13, 2011. Photograph by Joe Gorman, USGS.}




\section{Methods for Probability Analyses}

Selection of an appropriate probability distribution and the process for fitting parameters of the distribution may vary depending on the underlying characteristics of the data. For consistency in estimating flood frequencies, Federal agencies follow standard guidelines, known as Bulletin 17B (U.S. Interagency Advisory Committee on Water Data, 1982), which recommended the use of the log-Pearson type III (LPIII) distribution and the "method of moments" for estimating the distribution parameters (mean, standard deviation, and skewness of the data). For USGS streamgages, the peak streamflow and annual runoff data are available from the USGS National Water Information System database (U.S. Geological Survey, 2013).

This report section provides results of probability analyses for both peak streamflows and annual runoff volumes for streamgages considered by Holmes and others (2013). These probability analyses were done to incorporate streamflow data through 2011 using a standardized process, as described herein, for the purpose of broadscale characterization of the relative frequency of occurrence of the 2011 flooding in the Central United States. Results of the frequency analyses are not intended for site-specific applications, for which more rigorous analytical methods involving multiple approaches or reviews of previous probability analyses might be justified, as recommended in Bulletin 17B (U.S. Interagency Advisory Committee on Water Data, 1982). As such, site-specific applications could involve consideration of site-specific circumstances, which could include consideration of other published frequency analyses such as Parrett and Johnson (2003), Sando and others (2008), U.S. Army Corps of Engineers (2001, 2004, and 2010), and Westerman and others (2013).

In many previous flood reports (for example, Chin and others, 1975; Parrett and others, 1993; Holmes and Kupka, 1997), flood probabilities were expressed by listing T-year recurrence intervals for particular flood quantiles (for example, the "100-year flood"), which recently has been discouraged to avoid confusing the general public (Holmes and Dinicola, 2010). The use of an AEP percentage for a flood is instead recommended because of the clear communication that the peak streamflow is being characterized by its probability or chance of occurrence. Users can easily convert from the AEP to the T-year recurrence interval by simply taking the reciprocal of the AEP. For example, a 1-percent AEP flood corresponds to the streamflow magnitude that is equaled or exceeded by a probability (expressed as a decimal) of 0.01 in any given year. The reciprocal of 0.01 is 100 , thus the T-year recurrence interval for a 1-percent AEP flood is the 100-year flood.

The reliability of an AEP flood quantile from Bulletin 17B methods may be expressed as a "variance of prediction" that is computed by using the asymptotic formula given by Cohn and others (2001), with the addition of the mean-squared error of generalized skew (Griffis and others, 2004). The variance of prediction varies as a function of record length, the fitted flood-probability distribution parameters (mean, standard deviation, and weighted skew), and the accuracy of the method used to determine the regional skew component of the weighted skew.

Another way to obtain an AEP flood-quantile estimate, besides using Bulletin 17B methods, is to use regional regression equations (RREs), when available (http://water.usgs.gov/ software/nss.html). RREs are developed by using regression techniques that relate the flood-probability data for many streamgages in a particular region to basin characteristics of streams (Jennings and others, 1994). For any location along a stream (gaged or ungaged), a user can enter basin characteristics (drainage area, basin slope, and so on) as independent variables into RREs and compute various streamflow characteristics, such as the 1-percent AEP flood quantile. The variance of prediction from the regional regression is a function of the RRE and values of independent variables used to develop the streamflow estimate from the RRE. The variance generally increases with departure of actual values from mean values of the independent variables.

Holmes and others (2010) provided detailed discussions regarding expressing the accuracy of RREs in terms of equivalent years of record and suggested that weighting by variance provides a more natural characterization of the underlying uncertainty of the various streamflow estimates. The optimal estimate of the AEP flood quantile for a gaged site is determined by weighting the AEP flood-quantile estimate determined from the Bulletin 17B methods with the AEP flood quantile estimate determined from the RRE. The weights are inversely proportional to the variances of prediction (equation 1), yielding the weighted estimator:

$$
\log Q_{P, O P T}=\frac{\left(\operatorname{Var}[R R E] * \log Q_{P, L P I I}+\operatorname{Var}[L P I I I] * \log Q_{P, R R E}\right)}{(\operatorname{Var}[R R E]+\operatorname{Var}[L P I I I])}
$$

where

$Q_{P, O P T} \quad$ is the optimal estimate of AEP flood quantile for a particular probability of flooding $(p)$ (U.S. Interagency Advisory Committee on Water Data, 1982, appendix 8),

$\operatorname{Var}[R R E] \quad$ is the variance of the RRE estimate of the AEP flood quantile for a particular probability of flooding $(p)$,

$Q_{P, L P I I I} \quad$ is the Bulletin 17B method estimate of the AEP flood quantile for a particular probability of flooding $(p)$,

$\operatorname{Var}[L P I I I] \quad$ is the variance of the Bulletin 17B estimate of the AEP flood quantile for a particular probability of flooding $(p)$, and

$Q_{P, R R E} \quad$ is the RRE estimate of the AEP flood quantile for a particular probability of flooding $(p)$. 


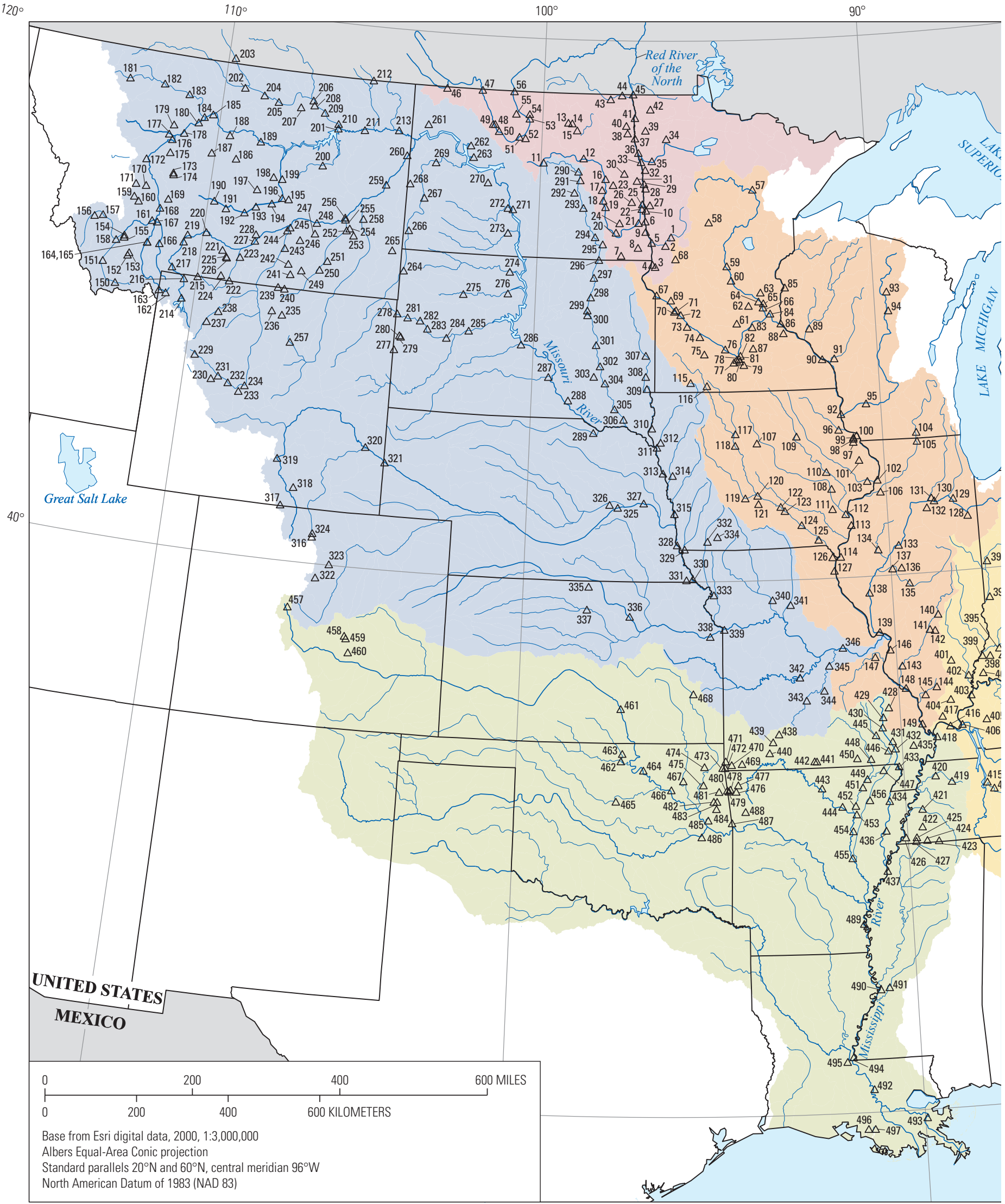

Figure 2. Locations of selected streamgages in the Central United States where flooding took place during February to September, 2011 (modified from Holmes and others, 2013). 


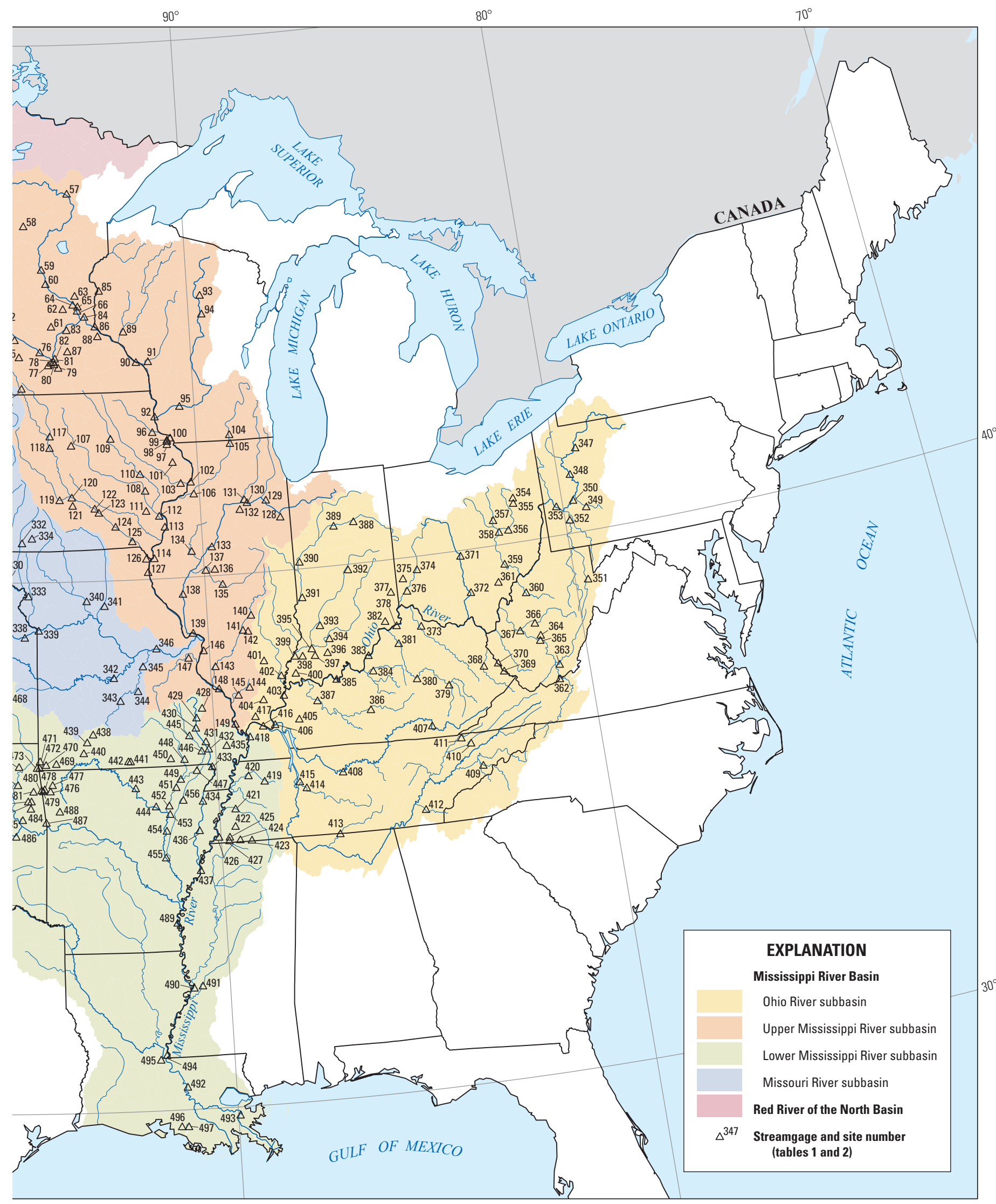

Figure 2. Locations of selected streamgages in the Central United States where flooding took place during February to September, 2011 (modified from Holmes and others, 2013).-Continued 
Data regarding AEPs for peaks during the 2011 floods are provided in table 1 (at the end of this report) for 369 streamgages in the Red, Souris, upper Mississippi, Missouri, Ohio, and lower Mississippi River Basins. Table 1 also includes data for previous streamflow maxima and rankings of the 2011 streamflow peaks that were previously reported by Holmes and others (2013). Streamgages were chosen either because the AEP for 2011 peak streamflow was anticipated to be less than 10-percent or to allow comparison with past major floods. Although desirable, weighting with data from RREs could not be used in computing optimal AEP flood-quantile estimates for many streamgages, most typically for regulated streamgages, where RREs are not applicable or not available. The table 1 listings for most streamgages include an AEP estimate for the 2011 flood peak and estimates of the AEP flood quantiles corresponding to the 2-percent, 1-percent, and 0.2-percent AEPs, along with their respective 95-percent confidence limits. The AEP estimate for each 2011 flood peak also includes lower and upper bounds for a "typical range" defined by 66.7 -percent confidence levels. This typical range is calculated using order statistics and is nonparametric, that is, it does not assume the same underlying LPIII statistical model that is used in fitting the at-site streamflow data for computing the flood-quantile estimates. Thus, for a streamgage where an individual flood magnitude (such as 2011) is especially large relative to the rest of a dataset, the LPIII model-based AEP estimate can lie outside the typical range of AEP for a flood of a given rank within a specified period of record.

AEPs for the 2011 flood peaks are not listed for 48 streamgages, of which AEP flood quantiles are not available for 15 streamgages. AEP flood quantiles are listed for 33 streamgages that were obtained only from RREs or that were extracted from previous floods studies (Parrett and Johnson, 2003; Sando and others, 2008; U.S. Army Corps of Engineers; 2001, 2004, and 2010; and Westerman and others, 2013) that are not inclusive of the 2011 streamflow peaks. Thus, estimation of AEPs from these flood quantiles would not be consistent with AEP estimates throughout the rest of table 1 .

For some streamgages in table 1, the number of streamflow peaks used for the AEP analysis was smaller than what was listed for the period of record. There are several possible reasons, of which the most common were use of a shortened analysis period to reflect current (and thus consistent) conditions relative to effects of regulation and exclusion of historic peaks from an analysis. For a few streamgages, the number of streamflow peaks used for the AEP analysis was larger than listed for the period of record, which most commonly was based on comparisons with records for nearby streamgages (U.S. Interagency Advisory Committee on Water Data, 1982). Other reasons for using more peaks for AEP analysis involve other record-extension procedures and adjustments associated with procedures involving use of historic peaks. Sando and others (2008) provided in-depth discussions of procedures used in a statewide peak-flow analysis for South Dakota streamgages. Those procedures are applicable for many of the
South Dakota streamgages listed in table 1 and also could be applicable for other streamgages in table 1 .

Flood-quantile estimates for annual runoff volumes corresponding to the 4-percent, 2-percent, 1-percent, and 0.2-percent AEPs, along with their respective 95-percent confidence limits, are listed in table 2 (at the end of this report) for 211 streamgages in the Red, Souris, upper Mississippi, Missouri, Ohio, and lower Mississippi River Basins. Table 2 also includes data for previous annual runoff maxima reported by Holmes and others (2013). Weighting with data from RREs could not be used in computing AEP flood quantiles for any of the streamgages because RREs for flood volume typically do not exist. Furthermore, regional skew coefficients do not exist for annual runoff, which precludes weighting of skew coefficients for individual streamgages. AEP ranges for the 2011 runoff volumes are provided (rather than specific AEP estimates) because specific AEP confidence limits for the 2011 runoff volumes cannot be determined.

For some of the 83 streamgages in table 2 that are common with streamgages in table 1, the number of years of annual runoff data is smaller than the length of the peak-flow record used for AEP analysis, most typically because of years when only peak streamflow was collected, interruptions in the continuous streamflow record, or regulation or diversions in the basin affecting what years were used for the peak-flow record analysis (as footnoted in table 1). The period of peakflow record considered for AEP analysis in table 1 typically was restricted to a period of consistent regulation conditions because of especially large effects that regulation can have on peak-flow conditions. In contrast, effects of regulation on annual runoff typically are small (especially relative to the high flows of interest) and often tend to be offsetting in an annual time series. Furthermore, effects of regulation may be small relative to the effects of the choice of an applicable probability distribution, which may not necessarily be lognormal for some annual runoff datasets. Thus, benefits from increased record length were considered to outweigh minor detriments from inconsistent regulation conditions.

\section{Results of Probability Analyses}

Figure 3 shows AEP ranges for peak streamflows during the 2011 floods for streamgages from table 1, and figure 4 shows AEP ranges for 2011 runoff volumes for streamgages from table 2. Inspection of figure 3 indicates that lowprobability peak streamflow (small AEPs or large recurrence intervals) occurred throughout large parts of the Mississippi River Basin in 2011. However, inspection of figure 4 indicates that the low-probability runoff volumes were concentrated in the northwestern part of the basin (Red, Souris, and Missouri River Basins); whereas, higher-probability runoff volumes were more prevalent throughout the southern and eastern parts of the basin.

Table 3 provides a summary of results of probability analyses for annual peak streamflows (table 1) and annual runoff volumes (table 2). Within the Red River of the North 


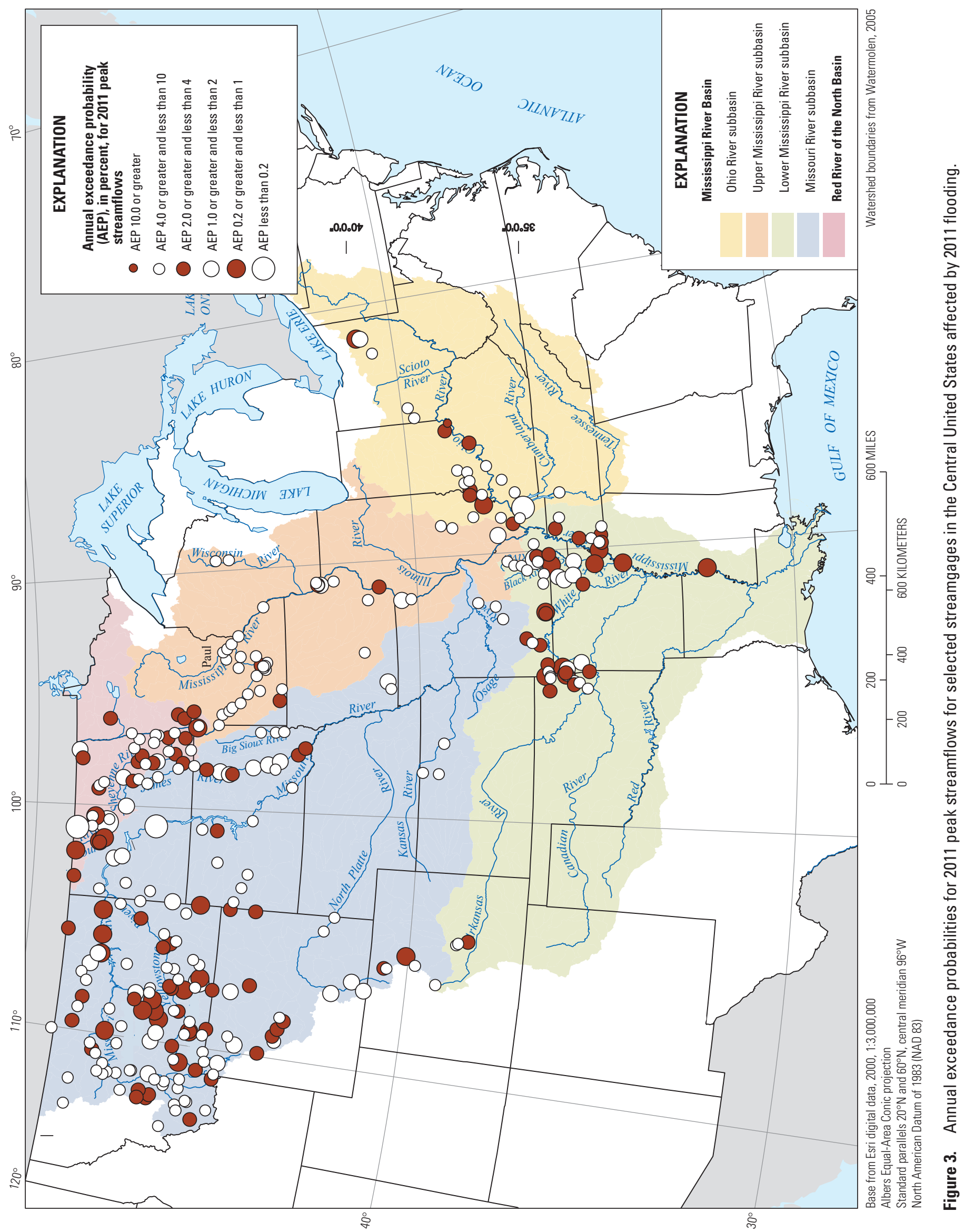




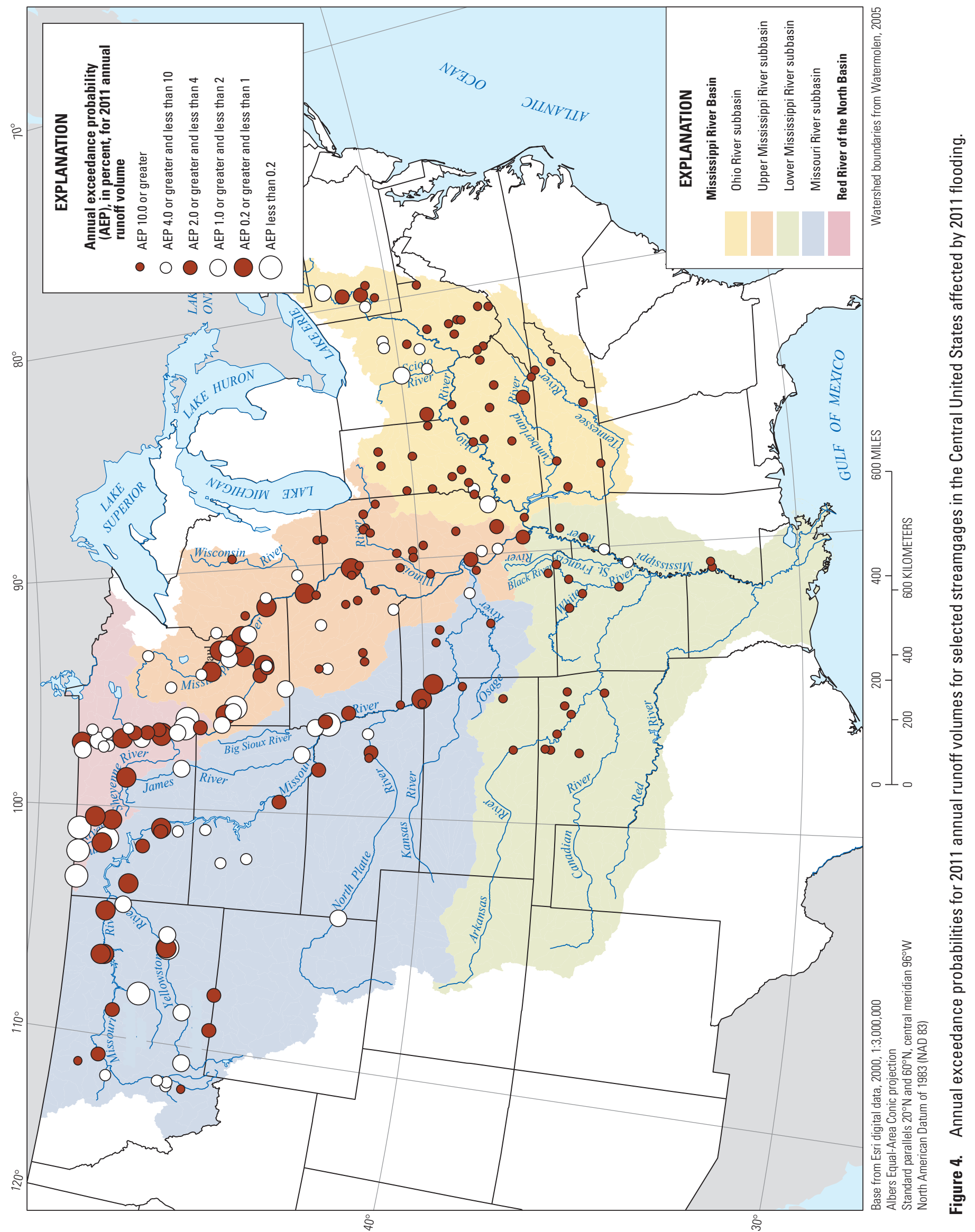


Basin, AEPs for 2011 peak streamflows were not exceptionally small, with only 1 of 29 streamgages with computed AEPs having a peak streamflow in the range of 0.2 to 1 percent and 4 in the range of 1 to 2 percent. AEPs for many of the 2011 runoff volumes were small; however, with 2 of 20 streamgages less than 0.2 percent, 5 streamgages in the range of 0.2 to 1 percent, and 4 streamgages in the range of 1 to 2 percent. Magnitudes of annual runoff volumes were exceptional, with all 11 streamgages with AEPs less than 2 percent eclipsing previous long-term (62 to 110 years) annual maxima by about one-third or more. Flooding was caused primarily by melting of a heavy snowpack, with most of the streamflow peaks occurring during April (Vining and others, 2013).

AEP estimates are not included in table 1 for 8 of the 37 Red River streamgages, all of which are along the main stem. One of these is streamgage 05054000 (at Fargo, N. Dak.), for which the largest flow in 111 years of peakstreamflow record $\left(29,500 \mathrm{ft}^{3} / \mathrm{s}\right.$ in 2009) surpassed the 2011 flow of $27,200 \mathrm{ft}^{3} / \mathrm{s}$. Neither of these exceptional values was considered within the U.S. Army Corps of Engineers (2001) hydrology report for the Red River, which is the source of the quantile estimates in table 1 . Those quantile estimates were developed through consideration of many relevant datasets, and in concurrence with other cooperating agencies (including USGS), to be used as a primary source of flood-probability information for the Red River. For this reason, AEPs for 2011 flood peaks are not listed for streamgage 05054000 and the seven other main-stem streamgages along the Red River.

The 2011 flooding in the Souris River Basin was exceptional from all standpoints. Six of 11 streamgages listed in table 1 for the Souris River Basin recorded flood peaks that were more than double the previous peak of record. These six streamgages were among the longest-term streamgages and had 75 to 108 years of peak-flow record. AEPs for these six streamgages were less than 1 percent (tables 1 and 3 ). AEPs for 2011 runoff volumes were less than 1 percent for all seven

Table 3. Summary of results of probability analyses for selected streamgages in the Central United States for annual peak streamflows (from table 1) and annual runoff volumes (from table 2).

\begin{tabular}{|c|c|c|c|c|c|c|c|c|c|}
\hline \multirow[b]{2}{*}{ Basin } & \multicolumn{6}{|c|}{$\begin{array}{l}2011 \text { annual peak streamflows, number of streamgages within annual } \\
\text { exceedance probability (AEP) range }\end{array}$} & \multirow[b]{2}{*}{$\begin{array}{c}\text { Number } \\
\text { of sites } \\
\text { with AEP }\end{array}$} & \multirow[b]{2}{*}{$\begin{array}{l}\text { AEP not } \\
\text { calcu- } \\
\text { lated }\end{array}$} & \multirow[b]{2}{*}{$\begin{array}{l}\text { Total } \\
\text { sites in } \\
\text { table } 1\end{array}$} \\
\hline & $\begin{array}{l}10.0 \text { or } \\
\text { greater }\end{array}$ & $\begin{array}{c}4.0 \text { or } \\
\text { greater } \\
\text { and less } \\
\text { than } 10\end{array}$ & $\begin{array}{l}2.0 \text { or } \\
\text { greater } \\
\text { and less } \\
\text { than } 4\end{array}$ & $\begin{array}{c}1.0 \text { or } \\
\text { greater } \\
\text { and less } \\
\text { than } 2\end{array}$ & $\begin{array}{c}0.2 \text { or } \\
\text { greater } \\
\text { and less } \\
\text { than } 1\end{array}$ & $\begin{array}{l}\text { Less } \\
\text { than } 0.2\end{array}$ & & & \\
\hline Red River of the North & 0 & 13 & 11 & 4 & 1 & 0 & 29 & 8 & 37 \\
\hline Souris River & 0 & 3 & 2 & 0 & 4 & 2 & 11 & 0 & 11 \\
\hline Upper Mississippi River & 0 & 33 & 5 & 3 & 0 & 0 & 41 & 12 & 53 \\
\hline Missouri River & 0 & 84 & 37 & 23 & 14 & 2 & 160 & 16 & 176 \\
\hline Ohio River & 1 & 15 & 5 & 1 & 2 & 1 & 25 & 1 & 26 \\
\hline Lower Mississippi River & 0 & 21 & 16 & 8 & 9 & 1 & 55 & 11 & 66 \\
\hline Total selected streamgages & 1 & 169 & 76 & 39 & 30 & 6 & 321 & 48 & 369 \\
\hline \multirow[b]{2}{*}{ Basin } & \multicolumn{6}{|c|}{$\begin{array}{l}2011 \text { annual runoff volumes, number of streamgages within annual } \\
\text { exceedance probability (AEP) range }\end{array}$} & \multirow[b]{2}{*}{$\begin{array}{c}\text { Number } \\
\text { of sites } \\
\text { with AEP }\end{array}$} & \multirow[b]{2}{*}{$\begin{array}{l}\text { AEP not } \\
\text { calcu- } \\
\text { lated }\end{array}$} & \multirow[b]{2}{*}{$\begin{array}{c}\text { Total } \\
\text { sites in } \\
\text { table } 2\end{array}$} \\
\hline & $\begin{array}{l}10.0 \text { or } \\
\text { greater }\end{array}$ & $\begin{array}{c}4.0 \text { or } \\
\text { greater } \\
\text { and less } \\
\text { than } 10\end{array}$ & $\begin{array}{c}2.0 \text { or } \\
\text { greater } \\
\text { and less } \\
\text { than } 4\end{array}$ & $\begin{array}{c}1.0 \text { or } \\
\text { greater } \\
\text { and less } \\
\text { than } 2 \\
\end{array}$ & $\begin{array}{c}0.2 \text { or } \\
\text { greater } \\
\text { and less } \\
\text { than } 1\end{array}$ & $\begin{array}{l}\text { Less } \\
\text { than } 0.2\end{array}$ & & & \\
\hline Red River of the North & 0 & 5 & 4 & 4 & 5 & 2 & 20 & 0 & 20 \\
\hline Souris River & 0 & 0 & 0 & 0 & 3 & 4 & 7 & 0 & 7 \\
\hline Upper Mississippi River & 26 & 12 & 5 & 6 & 10 & 1 & 60 & 0 & 60 \\
\hline Missouri River & 9 & 10 & 11 & 8 & 8 & 3 & 49 & 0 & 49 \\
\hline Ohio River & 40 & 6 & 4 & 3 & 0 & 0 & 53 & 0 & 53 \\
\hline Lower Mississippi River & 20 & 2 & 0 & 0 & 0 & 0 & 22 & 0 & 22 \\
\hline Total selected streamgages & 95 & 35 & 24 & 21 & 26 & 10 & 211 & $\mathbf{0}$ & 211 \\
\hline
\end{tabular}


streamgages in table 2. Magnitudes of annual runoff volumes also were exceptional, with all 7 streamgages exceeding previous maxima (record lengths 52 to 108 years), and 5 of the 7 streamgages exceeding by double or more. The heaviest flooding in this basin generally was in late June and was caused by heavy and widespread rainstorms that followed a heavy snowpack (Vining and others, 2013).

AEPs for peak streamflows in the upper Mississippi River Basin were not exceptional, with no AEPs less than 1 percent, 3 AEPs between 1 and 2 percent, and 38 AEPs of 2 percent or larger (table 3). AEPs for runoff volumes indicated somewhat less frequent recurrence, with 11 having AEPs of less than 1 percent, 11 having AEPs between 1 and 4 percent, and 38 having AEPs of 4 percent or larger. Most of the small AEPs for runoff volume were either along the main stem of the Mississippi River upstream from streamgage 05420500 (at Clinton, Iowa) or in tributaries upstream from streamgage 05331000 (at Saint Paul, Minnesota). The 2011 runoff volume for this streamgage exceeded the previous record (112 years of record) by about 24 percent. Moving downstream, the next three main-stem streamgages $(05344500,05378500$, and 05389500$)$ exceeded long-term records by about 4 to 13 percent (table 2 ).

The Missouri River upstream from Gavins Point Dam at Yankton, South Dakota, is regulated by a series of six large multiuse main-stem dams constructed between 1933 and 1963 to "be operated for the purposes of flood control, navigation, irrigation, power, water supply, water quality control, recreation, and fish and wildlife" (U.S. Army Corps of Engineers,
2006). The total system storage for the six main-stem reservoirs is 73.1 million acre-feet (U.S. Army Corps of Engineers, 2012). Many other dams provide additional regulation on various tributaries throughout the Missouri River Basin.

An especially newsworthy feature of the 2011 flooding in the Central United States was prolonged flooding along the main stem of the Missouri River downstream from Garrison Dam (upstream from Bismarck, N. Dak.) and extending downstream throughout the length of the Missouri River. The 2011 runoff volume for streamgage 06342500 (at Bismarck) exceeds the previous (1975) maximum by about 50 percent, with an associated AEP in the range of 0.2 to 1 percent (table 2). Similarly, new annual runoff records (by about 37 to 42 percent, relative to previous maxima from 1997) were set for the next three downstream main-stem streamgages (06486000, at Sioux City, Iowa; 06813500, at Rulo, Nebraska; and 06818000, at St. Joseph, Missouri). AEPs for the 2011 runoff volumes are less than 1 percent for all three streamgages. However, the 2011 runoff volume for streamgage 06934500 (at Hermann, Mo.) is only about 76 percent as large as the volume for the record flow of 1993 and has an associated AEP exceeding 4 percent. AEPs are not listed for peak streamflows for streamgage 06486000 (at Sioux City, Iowa) and downstream main-stem streamgages because as previously described for the Red River Basin, the flood quantiles in table 1 are from an earlier U.S. Army Corps of Engineers (2004) report.

\section{Flooding along the Red River of the North in Grand Forks, North Dakota, April 13, 2011. Photograph by Brent Hansen, USGS.}


Table 3 indicates 39 Missouri River streamgages with peak-streamflow AEPs of less than 2 percent, with all but 16 of these (table 1) occurring between streamgages 06115200 (near Landusky, Montana) and 06342500 (at Bismarck, N. Dak.) and all but 3 of 16 with AEPs of 1 percent or less in this reach. Similarly 12 of 19 streamgages with runoff-volume AEPs less than 2 percent are in the same reach, with 4 of the other 7 streamgages along the main stem of the Missouri River (table 2). The exceptional runoff and flooding in this reach was caused by a combination of melting of a heavy snowpack followed by exceptionally heavy and widespread rainstorms in May and June (Vining and others, 2013).

Missouri River flooding was less exceptional upstream from Landusky, Mont. (streamgage 06115200), where the 1975 runoff volume exceeded the 2011 volume by 3 percent. Four main-stem streamgages upstream from Landusky (all in Montana; 06054500, at Toston; 06090300, near Great Falls; 06090800, at Fort Benton; and 06109500, at Vergelle) have peak-streamflow AEPs exceeding 4 percent. The 2011 runoff volume for long-term streamgage 06090800 (at Fort Benton) exceeds the previous (1894) maximum by about 3 percent; however, the associated AEP is between 2 and 4 percent because of the distribution of the annual-volume dataset.

Within the Ohio River Basin, most of the streamflow peaks occurred during early March to early May, with associated AEPs and runoff-volume AEPs that are not exceptional (table 3). For the Ohio River just upstream from the Mississippi River confluence (streamgage 03611500 at Metropolis, Illinois), the 2011 streamflow peak ranks fourth in 83 years of record, with an AEP of 4.0 percent (table 1), and the 2011 runoff volume ranks 26 of 83, with an AEP of greater than 10 percent (table 2). Throughout the basin, peak-streamflow AEPs are less than 2 percent for only four streamgages, and runoff-volume AEPs are less than 2 percent for only three streamgages.

Especially large streamflow peaks occurred along the lower Mississippi River during May. The largest streamflow peak in 91 years was recorded on May 17 for streamgage 07289000 (at Vicksburg, Mississippi), with an associated AEP of 0.8 percent. Annual streamflow peaks were recorded about a week earlier at two upstream Mississippi River streamgages (07032000, at Memphis, Tennessee and 07047970, at Helena, Arkansas), which exceeded previous maxima by about 8 and 7 percent, respectively, in 83 and 132 years of record, and with associated AEPs of less than 1 percent. Annual streamflow peaks also were recorded about another week earlier at streamgages along the Ohio and upper Mississippi Rivers. The St. Francis and Black Rivers, which have long peak-flow records, also had low-probability floods (AEPs less than 10 percent for 8 of the 10 streamgages on these rivers between station numbers 07036100 and 07072500 ). Low-probability peaks also were recorded for several streamgages along the Illinois River; however, associated periods of record generally are short. Of 55 lower Mississippi River streamgages considered for AEP analysis for peak streamflow, 10 had AEPs less than 1 percent (table 3). AEPs for 2011 runoff volumes for all of the lower Mississippi River streamgages were larger than 4 percent, which resulted from extensive drought conditions in areas to the west. For water year 2011, the Arkansas River at Tulsa, Oklahoma (streamgage 07164500) recorded the smallest runoff volume in 86 years of record, and other streamgages along the Arkansas, Canadian, and Big Black Rivers recorded unusually small runoff volumes.

\section{Trends in Peak Streamflows and Annual Runoff Volumes}

Knowledge regarding streamflow trends can be useful to water-resource and water-hazard planners and managers for many purposes. Various investigators have suggested trends in both peak streamflows and annual runoff volumes for areas that include the Central United States (Anderson and others, 2008; Hirsch, 2011; Holmes and others, 2010). This report section provides results of trend analyses for peak streamflows and annual runoff volumes for selected streamgages considered in tables 1 and 2 . The trend analyses were done to provide a broadscale perspective regarding trends for these specific streamgages, which were selected by Holmes and others (2013) to characterize flooding conditions in the Central United States. As such, most of these streamgages recorded high-flow conditions in 2011. Trend analysis emphasizing streamgages that had 2011 flooding has the potential to produce a small bias towards upward trends (as opposed to downward trends). Thus, results of the trend analyses should be considered a broadscale characterization, as opposed to a rigorous study of trends that would include in its design the selection of streamgages that would eliminate any artificial bias.

\section{Methods for Trend Analyses}

Annual time-series data for peak streamflows (table 1) and annual runoff volumes (table 2) were analyzed to determine the presence and associated magnitudes of trends through time for selected streamgages. Methods of analysis followed those of Holmes and others (2010), who reported on trends in peak streamflows relative to 2008 flooding in the Midwestern United States. Only trend magnitudes were computed with no effort to include null hypothesis significance testing, as much discussion in recent literature has focused on problems with null hypothesis significance testing (Nichols, 2001) and the issue of long-term persistence (Cohn and Lins, 2005) that may be erroneously taken as statistically significant trends. The omission of significance testing is an indication that results are reported for descriptive and comparative purposes, and may suggest avenues for future detailed research.

Trend magnitudes were computed based on the Sen slope estimator (Sen, 1968) using the MAKESENS application from the Finnish Meteorological Institute (Salmi and others, 2002). 


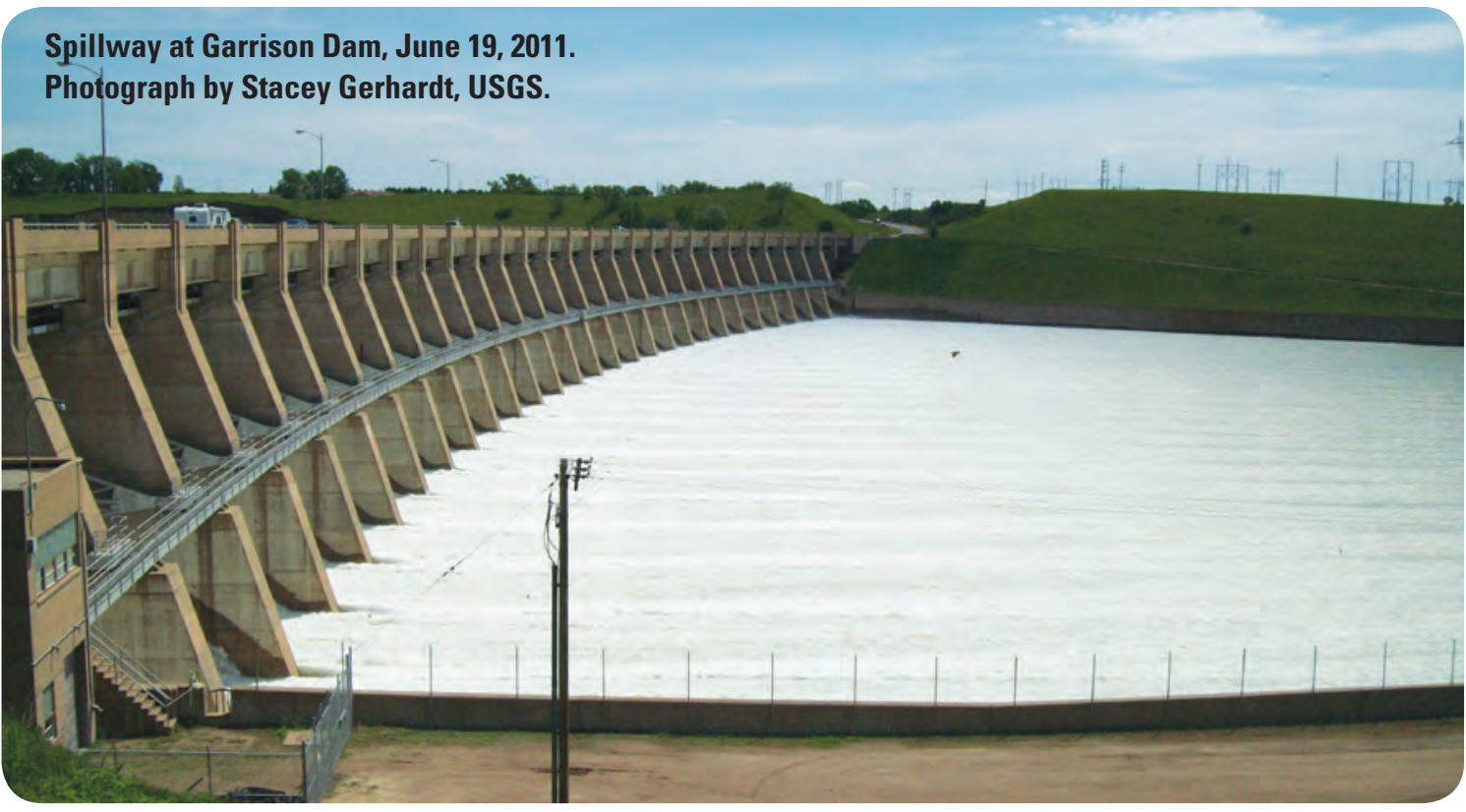

Powerhouse (left) and spillway (right) at Fort Randall Dam, June 9, 2011. Photograph by Joel Petersen, USGS.

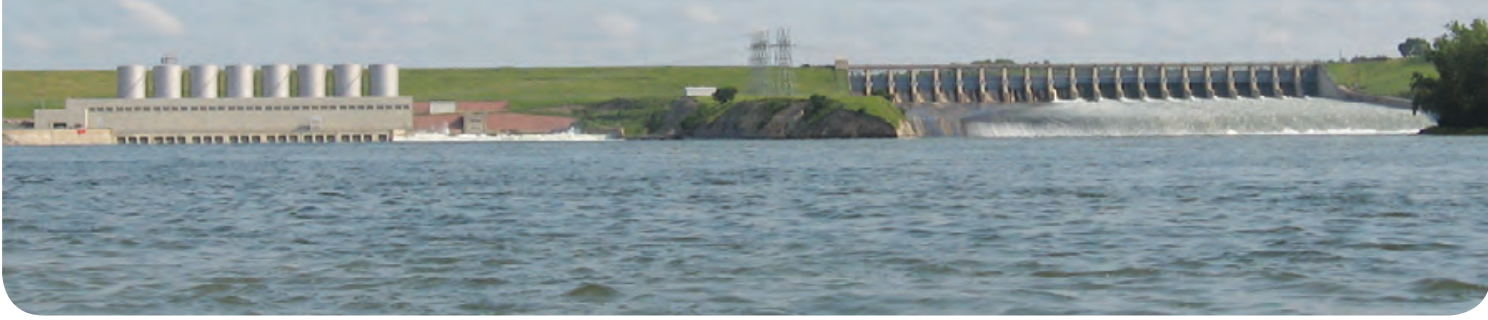

Oahe Dam (powerhouse in background), June 7,

2011. Photograph by Joel Petersen, USGS.

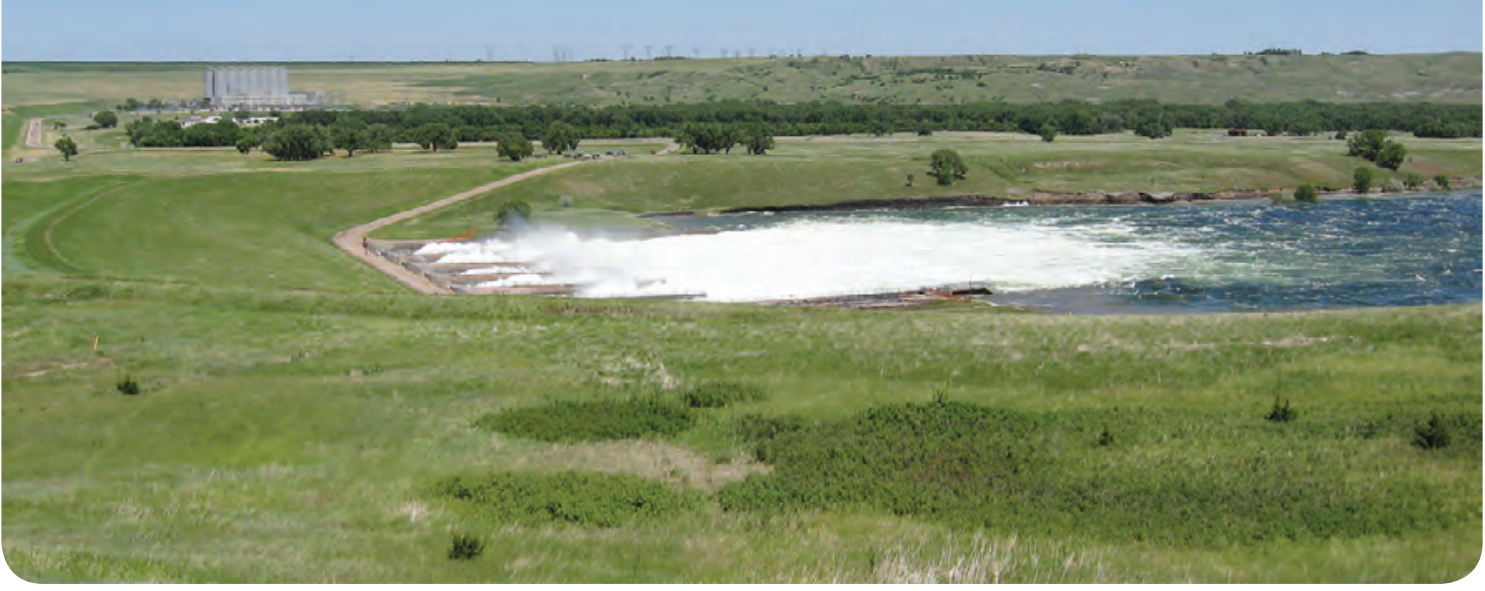

Photographs showing large water releases from three of the Missouri River main-stem dams 
The Sen slope, also known as the Kendall-Theil robust line, is a nonparametric estimator of trend magnitude slope for a univariate time series when the time interval is constant (equally spaced), as shown in equation 2.

$$
f(t)=M_{q} t+B
$$

where

$$
\begin{aligned}
& f(t) \text { is the increasing or decreasing function of } \\
& M_{q} \quad \text { is Sen slope (trend magnitude), } \\
& t \text { is time, and } \\
& B \text { is a constant. }
\end{aligned}
$$

The Sen slope is the median slope for all pairwise comparisons, with each pairwise difference divided by the number of years separating the records. To determine the Sen slope estimate in equation 2, slopes of all data pairs are calculated as shown in equation 3 .

$$
M_{j, k}=\frac{x_{k}-x_{j}}{\Delta t_{j, k}} \quad \text { for } j=1, \ldots, n-1 ; j<k \leq n
$$

where

$$
\begin{aligned}
M_{j, k} & \text { is slope between data points } x_{j} \text { and } x_{k}, \\
x_{j} & \text { is data measurement at time } j, \\
x_{k} & \text { is data measurement at time } k \text {, and } \\
\Delta t_{j, k} & \text { is change in time between observations. }
\end{aligned}
$$
$M_{j, k}$.

The Sen slope, $M_{q}$, is equal to the median value of all the

\section{Results of Trend Analyses}

Trends in peak streamflow were analyzed for 98 of the 321 streamgages listed table 1. Sites and periods of record considered were selected to be parallel with similar analyses by Holmes and others (2010) for 2008 flooding in the Midwestern United States, who used criteria outlined by Hodgkins and others (2007). Those criteria stipulated that streamgages must have at least 50 years of concurrent data with no more than 5 percent missing, and that streams must not be regulated by major dams or substantial regulating structures. The 50 -year criterion was arbitrary and was modified to a 54-year criterion (1958 to 2011) for this analysis, to allow direct comparison with sites common to those reported by Holmes and others (2010). Of 369 streamgages listed in table 1, 98 met these criteria (denoted by italics in table 1) and 271 were eliminated from the analysis.

For comparison of streamgages with varying basin sizes, the Sen slope for each streamgage was divided by the median annual peak streamflow, which provides scaling relative to the median annual peak streamflow for each streamgage. Results of analyses from 1958 to 2011 (scaled by median annual peak flood streamflow) are summarized in table 4 , which indicates 67 streamgages with upward trends, 31 with downward trends, and zero with no trend. Examination of figure 5 indicates a dichotomous spatial distribution in trends, with most streamgages in the northwestern part of the study area having either downward trends or small upward trends and most streamgages in the eastern part of the study area having upward trends. A cluster of streamgages in eastern North Dakota, eastern South Dakota, and southern Minnesota has consistently large upwards trends. Upward trends are somewhat smaller and less consistent (including a few downward trends) for streamgages southeast of this cluster.

Table 4. Results of trend analyses (1958 to 2011) for selected streamgages in the Central United States affected by 2011 flooding.

$[\mathrm{N}$, number of streamgages in the slope range $]$

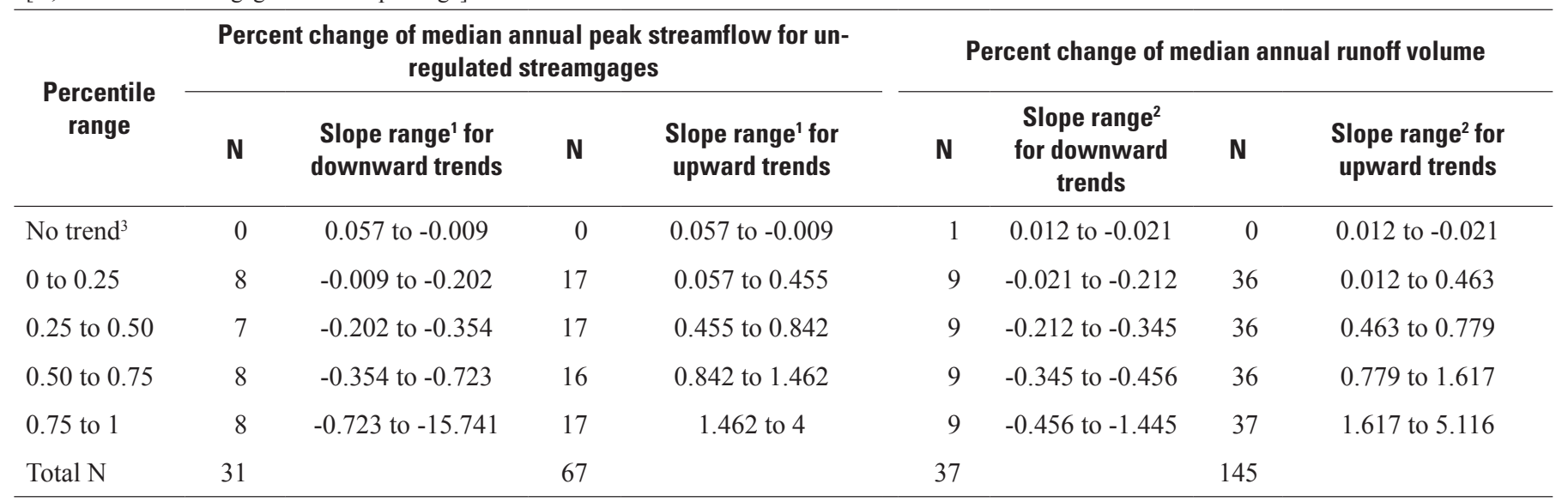

${ }^{1}$ Sen slope divided by the median annual peak streamflow (in percent change, per year).

${ }^{2}$ Sen slope divided by median annual runoff volume (in percent change, per year).

${ }^{3}$ The slope range for no trend brackets zero and is listed under both downward and upward trends. 


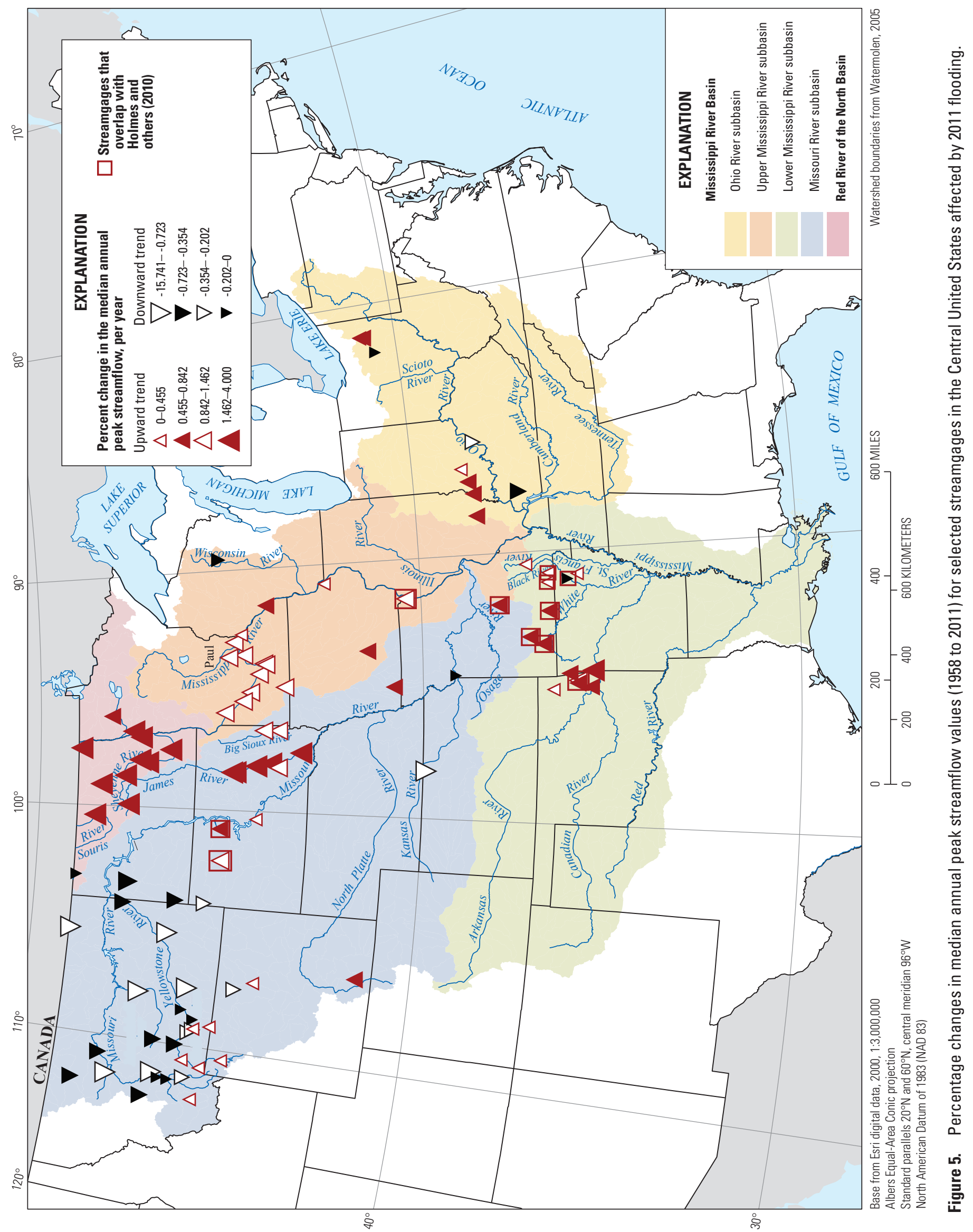




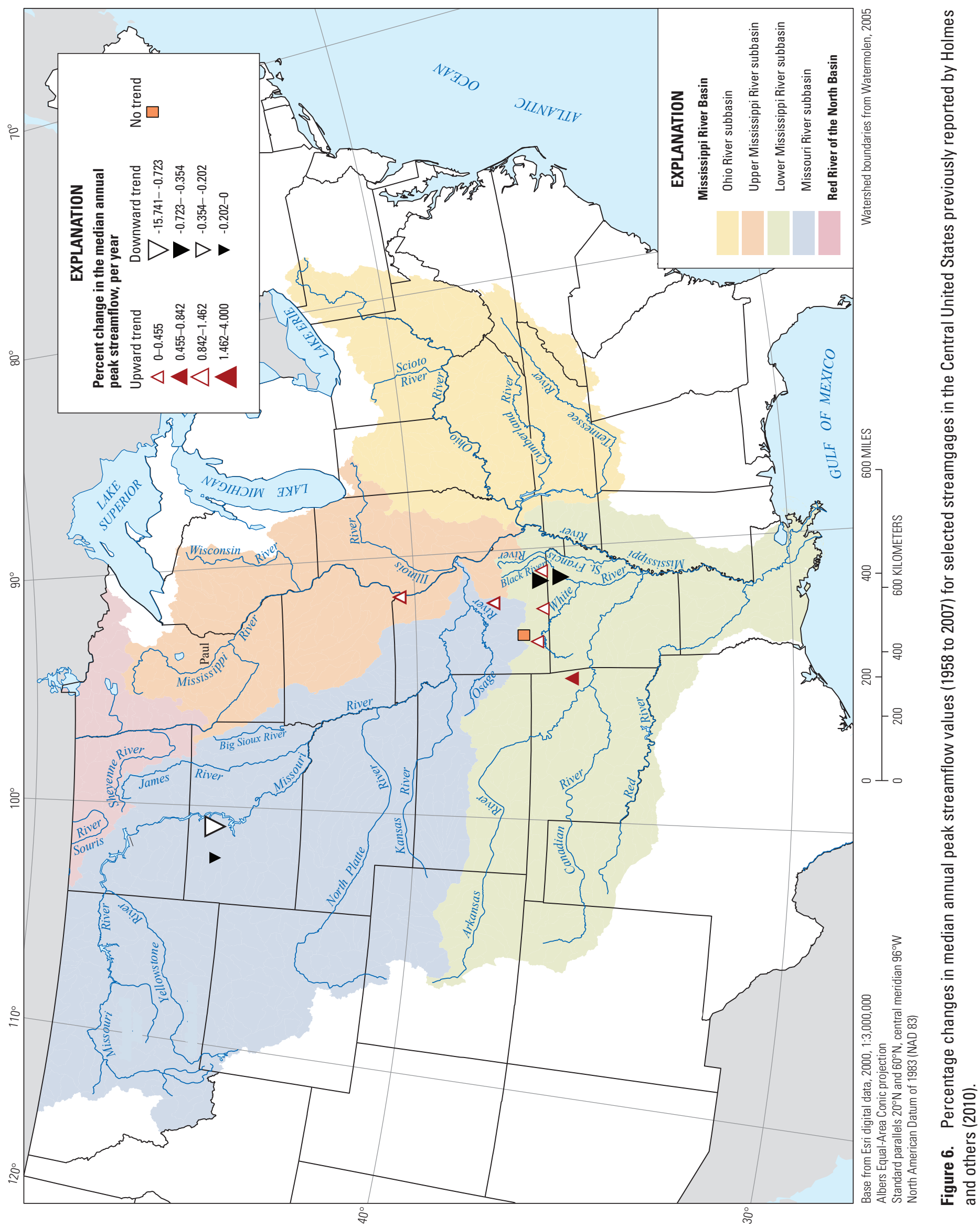




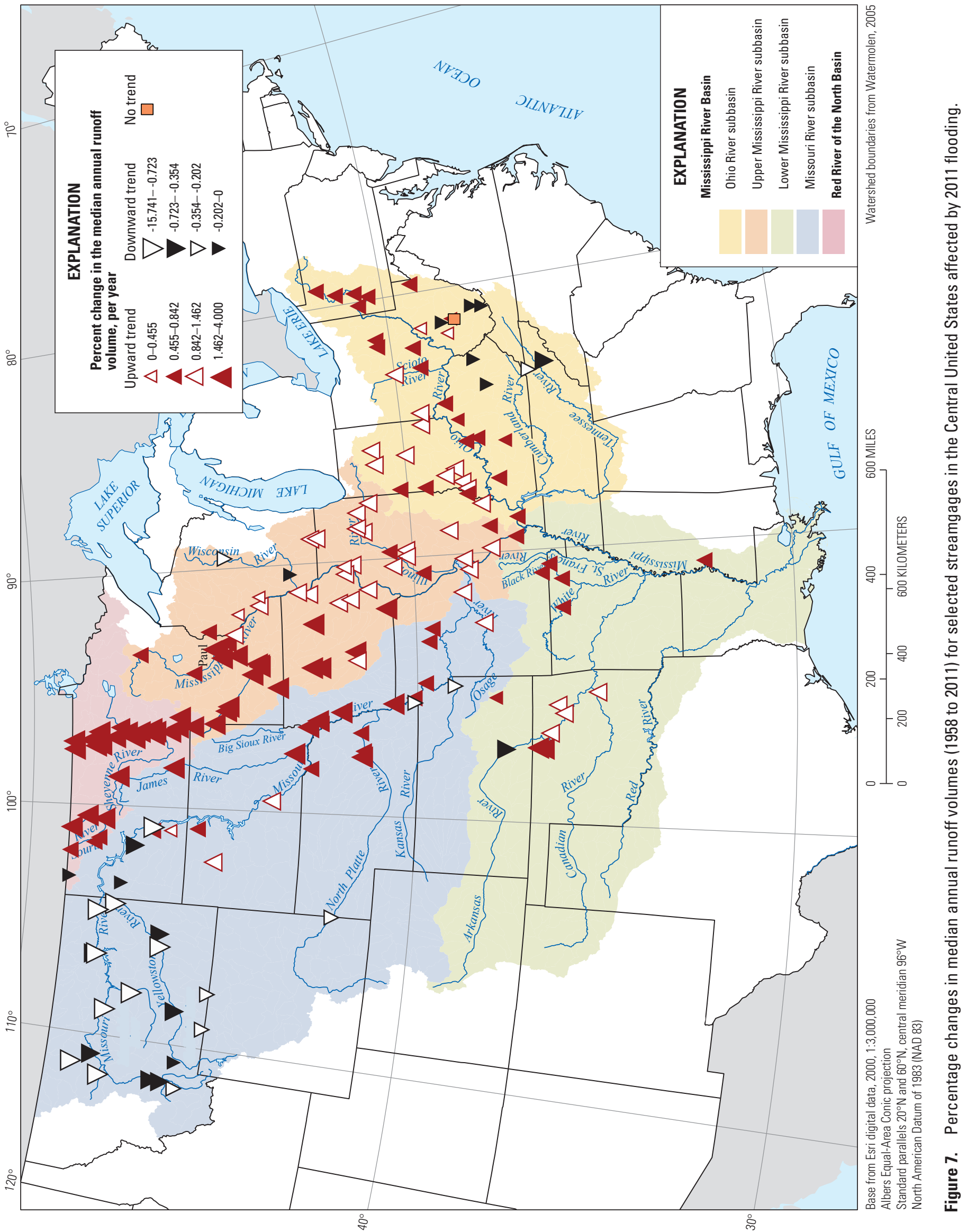


annual runoff volumes are indicative of regional-scale trends that cannot be ubiquitously affected by short-term (year-toyear) variability in streamflow. Trends in peak streamflow were analyzed only for unregulated streamgages having records dating back to at least 1958 , which restricted analyses to only 98 of the streamgages in table 1 . However, trends in annual runoff volume were analyzed for 182 streamgages in table 2. A more comprehensive analysis of trends could have included many additional streamgages. For example, figure 7 shows only two streamgages along the James River with upward trends, one in South Dakota and one just north of the North Dakota/South Dakota state line. In contrast, figure 5 shows five streamgages along the lower James River in South Dakota, of which four were not included in table 2. A more comprehensive analysis of trends also could have included additional years of record for many streamgages, as shown by examples (figs. 8 and 9) for the James River near Scotland, S. Dak. (streamgage 06478500), which was analyzed for trends in both peak streamflow and annual runoff volume.
Two large closed-basin lake systems, Devils Lake in North Dakota and the Waubay Lakes Chain in South Dakota, provide additional perspectives regarding hydrologic trends in the northern part of the area for which generally upward trends in peak streamflows and annual runoff volumes are indicated (figs. 5 and 7). The Waubay Lakes Chain (not shown) is located in a closed basin in northeastern South Dakota between headwaters of the Big Sioux, James, Little Minnesota, Sheyenne, and Red Rivers. Available lake-level records (South Dakota Department of Environment and Natural Resources, 2013) date back to only 1959 (fig. 10); however, flooding of several small towns and numerous homesteads indicates unprecedented lake levels dating back to the late 1800s (Niehus and others, 1999a; Niehus and others, 1999b). Extensive documentation exists (Vecchia, 2008; North Dakota State Water Commission, 2013) regarding long-term lake-level trends for Devils Lake (not shown), which is located just north of the Sheyenne River in northeastern North Dakota.

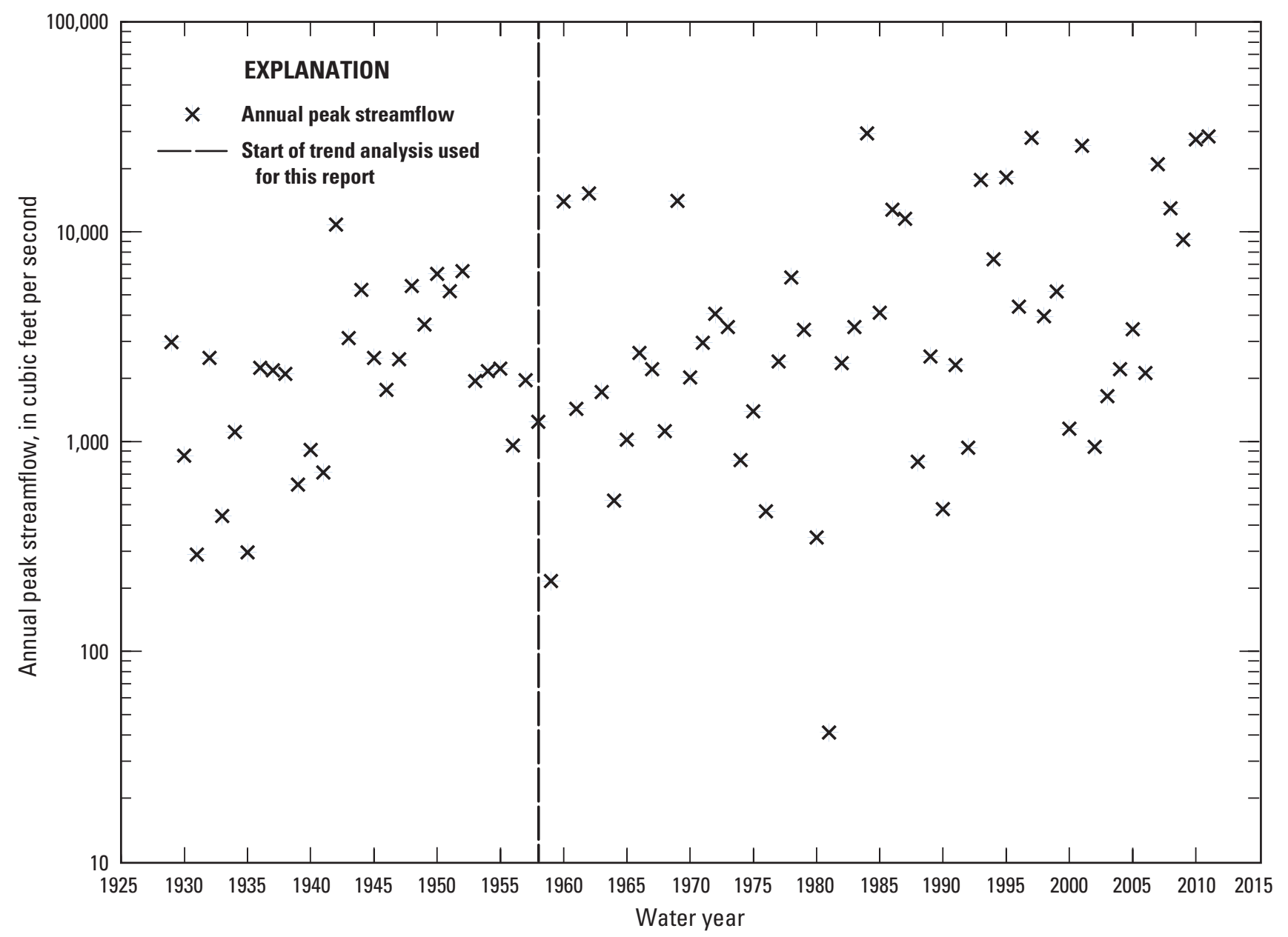

Figure 8. Time-series (1929 to 2011) record of annual peak streamflow for the James River near Scotland, South Dakota (streamgage 06478500). 


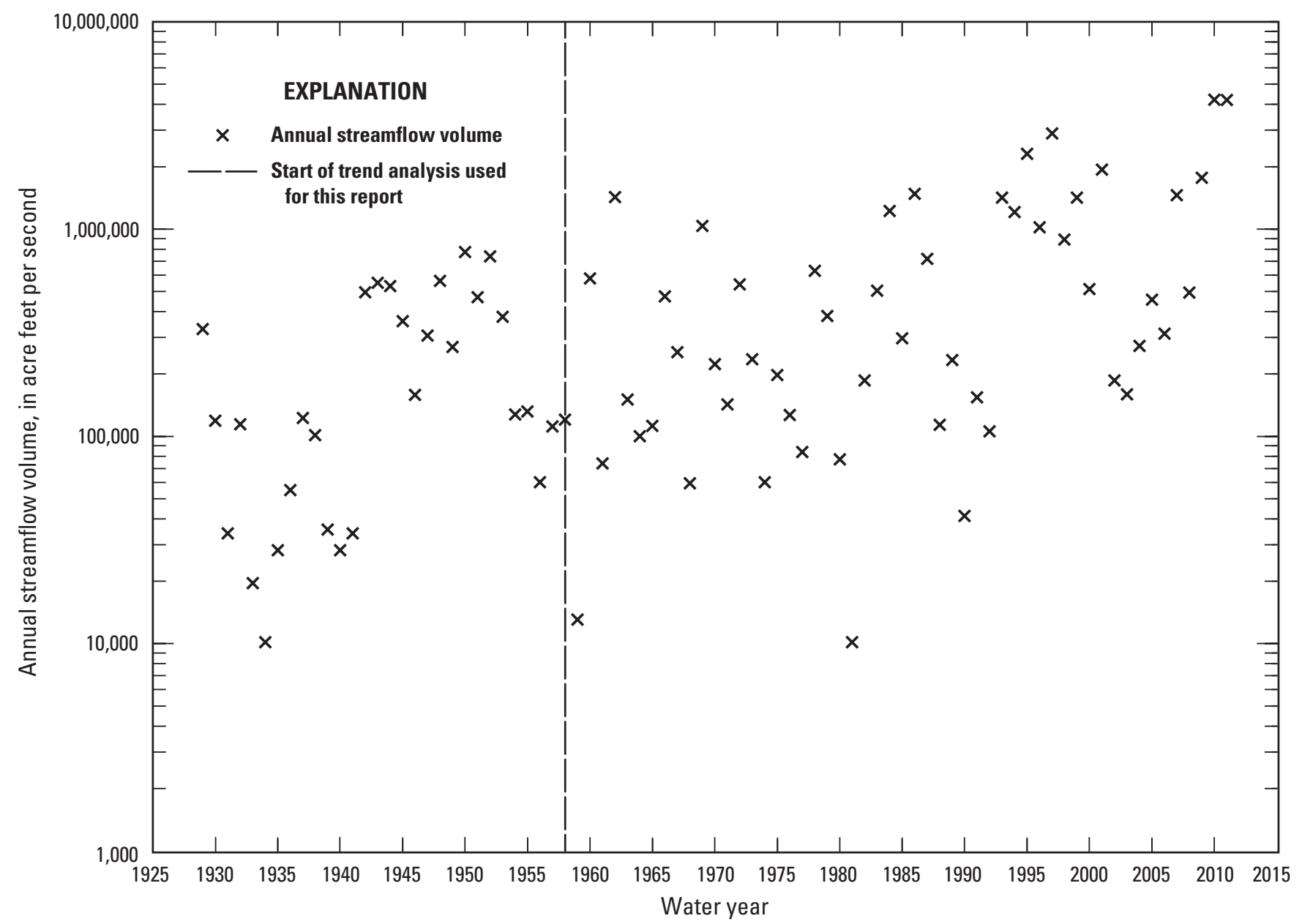

Figure 9. Time-series (1929 to 2011) record of annual runoff volume for the James River near Scotland, South Dakota (streamgage 06478500).

Figure 11 indicates about 70 years of generally downward trends followed by about 70 years of generally upward trends.

Collectively, the two closed-basin systems (figs. 10 and 11) indicate substantial changes throughout time in the lakesystem water balances, essentially the differential between lake inflows and evaporation. Recent upward trends in both closed-basin systems have been driven by especially wet climatic conditions that began in the early 1990s and have persisted at least sporadically ever since. The wet climatic conditions have been part of regional-scale conditions that have driven the peak-streamflow and annual-volume trends in the Central United States.

Additional perspectives are provided by figures 12 and 13 , which summarize information regarding annual time series for both peak streamflow and runoff volumes for streamgages that were considered in the trend analyses (figs. 5 and 7). Figures 12 and 13 show "normalized" sums of ranks (smallest to largest) for each of the six individual subbasins that were considered, as well as plots showing cumulative data for the combined subbasins (all streamgages for which the trend analyses were performed). The sums were normalized by dividing the sum of the ranks for each year by the number of streamgages in each river basin for which a value was available. The criterion for trend analysis required availability of at least 51 years out of a maximum of 54 years of record (1958 to 2011) for consideration. Using the Red River of the North as an example, the normalized sum of ranks for 2011 is about 53 for both the peaks and the runoff volumes, which indicates that 2011 was the largest, or nearly the largest value, for all of the streamgages considered, which is consistent with data presented in tables 1 and 2 .

Figures 12 and 13 include LOcally WEighted Scatterplot Smoothing (LOWESS) curves (Helsel and Hirsch, 2002), which emphasize the shape of a relation between two variables without assuming linearity or normality. The LOWESS curves 


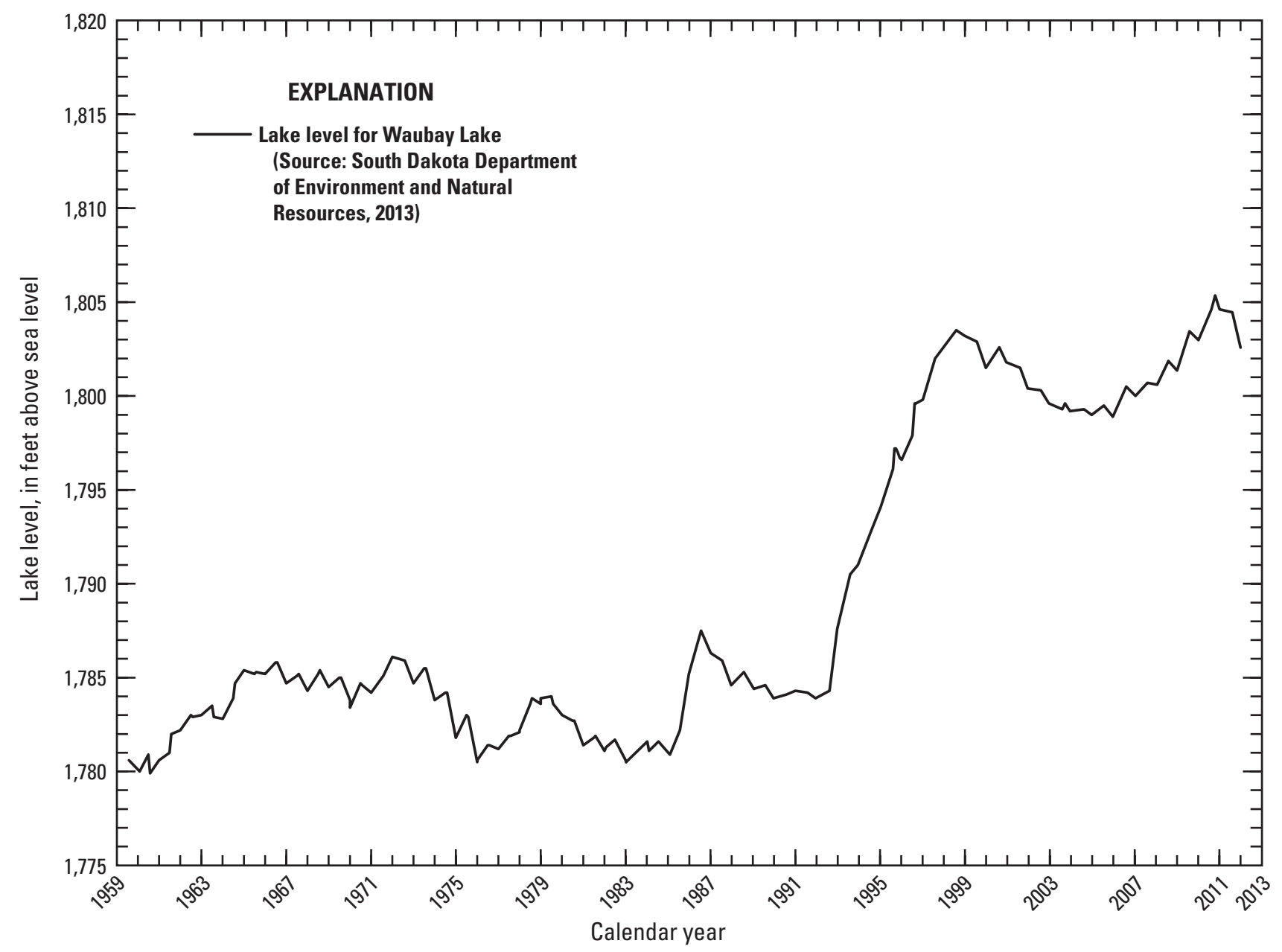

Figure 10. Lake-level record for Waubay Lake within the Waubay Lakes Chain in northeastern South Dakota.

also provide a robust description of the data pattern or trend (Helsel and Hirsch, 2002).

The LOWESS curves (figs. 12 and 13) are useful in pointing out periods of short-term trends and especially in combination with the normalized summed ranks, clearly depict primary regional-scale periods of relatively high or low streamflow conditions. Trends for all Red River of the North streamgages were consistently upwards and large for peak streamflows and annual runoff volumes (figs. 5 and 7), which is consistent with the LOWESS curves. In comparison, trends for the nearby Souris River Basin were smaller and less consistent, with one downward trend among streamgages analyzed for peak streamflows and for runoff volumes.

Although only two streamgages were analyzed for trends in peak streamflows, the regional patterns depicted by figure 12 are similar to those for the runoff volumes (fig. 13), with relatively small normalized sums for peak streamflows and runoff volumes recorded before 1969. The period of 1969 to 1979 included relatively high-flow conditions, with many of the previous maxima for peak streamflows and runoff volumes occurring during this period (tables 1 and 2). This was followed by a decade or more of relatively low-flow conditions, which culminated in particularly low annual runoff volumes for the late 1980s and early 1990s. Generally similar but dampened short-term trends for this period also are apparent for the Red River.

The LOWESS curves for the upper Mississippi River Basin are consistent with the trend analyses for both peak streamflows and runoff volumes (figs. 5 and 7), which are generally upwards, but as with the Souris River Basin, less strongly than for the Red River. The normalized summed ranks clearly indicate 1993 as an outstanding year for peak streamflows and as the most outstanding year for runoff volume in the upper Mississippi River Basin. Relatively large 


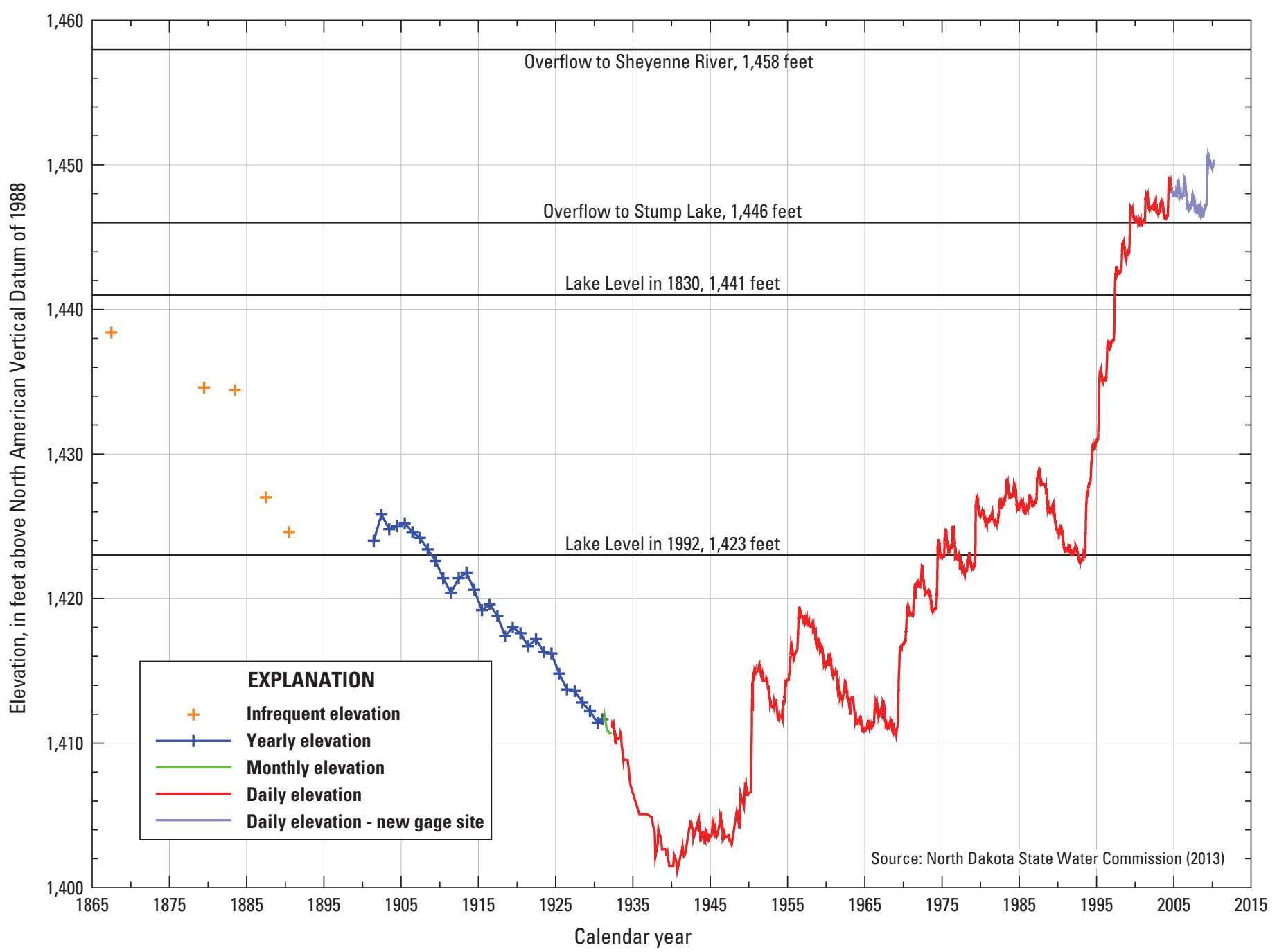

Figure 11. Lake-level record for Devils Lake in northeastern North Dakota.

peak streamflows and runoff volumes for 1993 also are apparent for the Red River and lower Mississippi River Basins, with 1993 essentially marking the start of the particularly wet climatic conditions that have driven the rising lake levels for the Waubay Lakes Chain and Devils Lake (figs. 10 and 11). Trends for both peak streamflows and runoff volumes for the Ohio and lower Mississippi River Basins generally were not as strongly upwards as for several of the other river basins, as reflected by the LOWESS curves.

For the Missouri River Basin, major extended drought periods are clearly apparent for about 1988 to 1992 and 2000 to 2007 (fig. 13). Relatively dry conditions for both periods are apparent to at least some extent for all of the other river basins and also are reflected in the cumulative plot for all streamgages. The cumulative plot also indicates a preponderance of large runoff volumes for the period of about 2007 or 2008 through 2011, and for the mid-1990s, as reflected in the plots for the individual river basins. A similar preponderance of large peak streamflows during these periods also is apparent for the cumulative plot for peak streamflows (fig.12), but is less pronounced for some of the individual river basins.

Trends in peak streamflows and annual flow volumes can be affected by climatic conditions and by land-use changes such as steadily increasing urbanization or large-scale changes in agricultural practices. The relative preponderance of highflow conditions since about 1993 closely corresponds with the lake-level records for Devils Lake and the Waubay Lakes Chain, which provide somewhat of a physical measure of changing water-balance conditions for the contributing drainage areas and collectively provide an indicator of regionalscale conditions that for these closed basins probably are driven primarily by climatic conditions. 

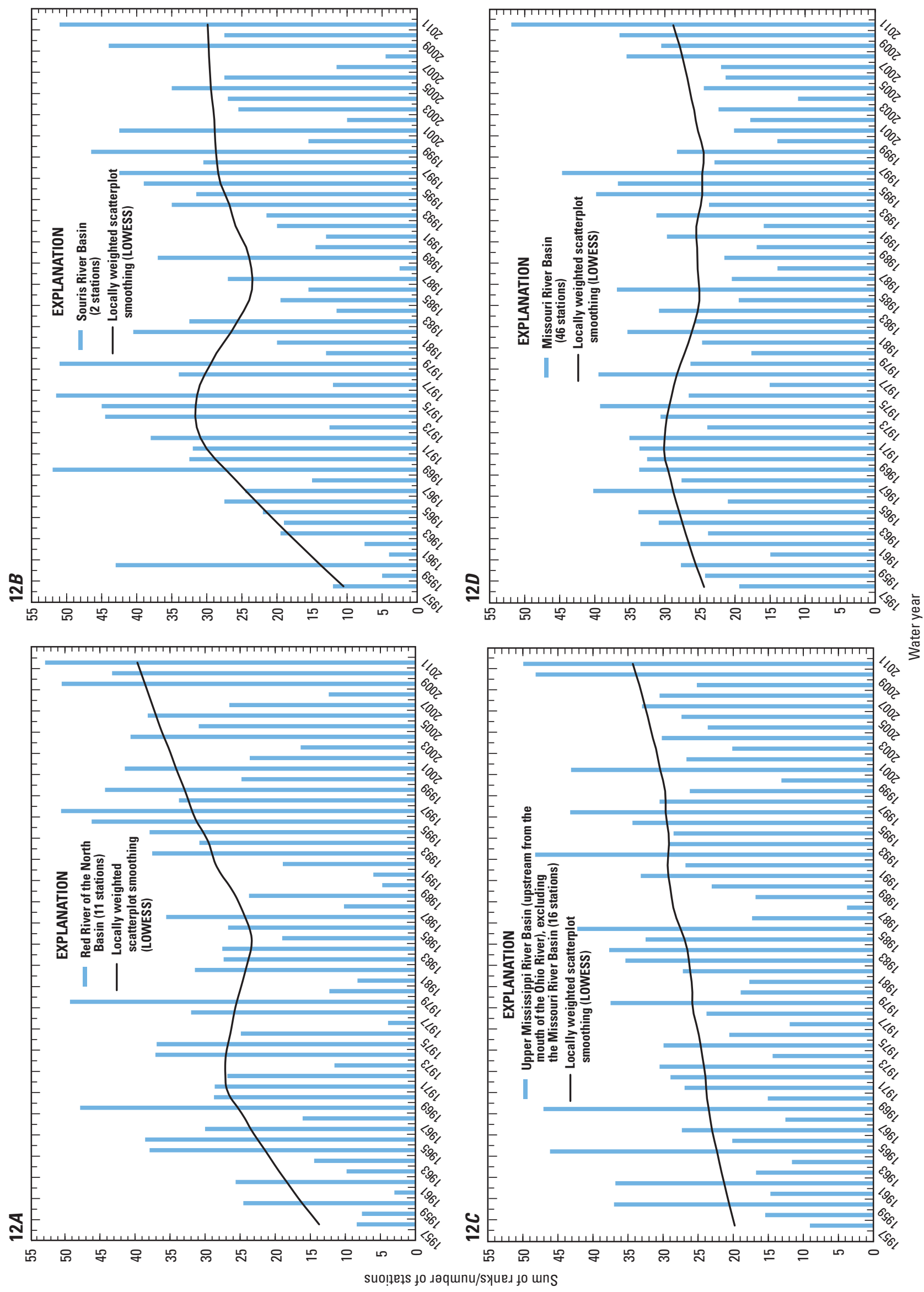

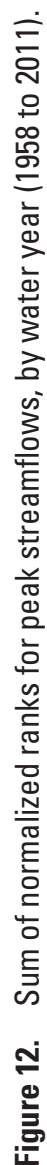



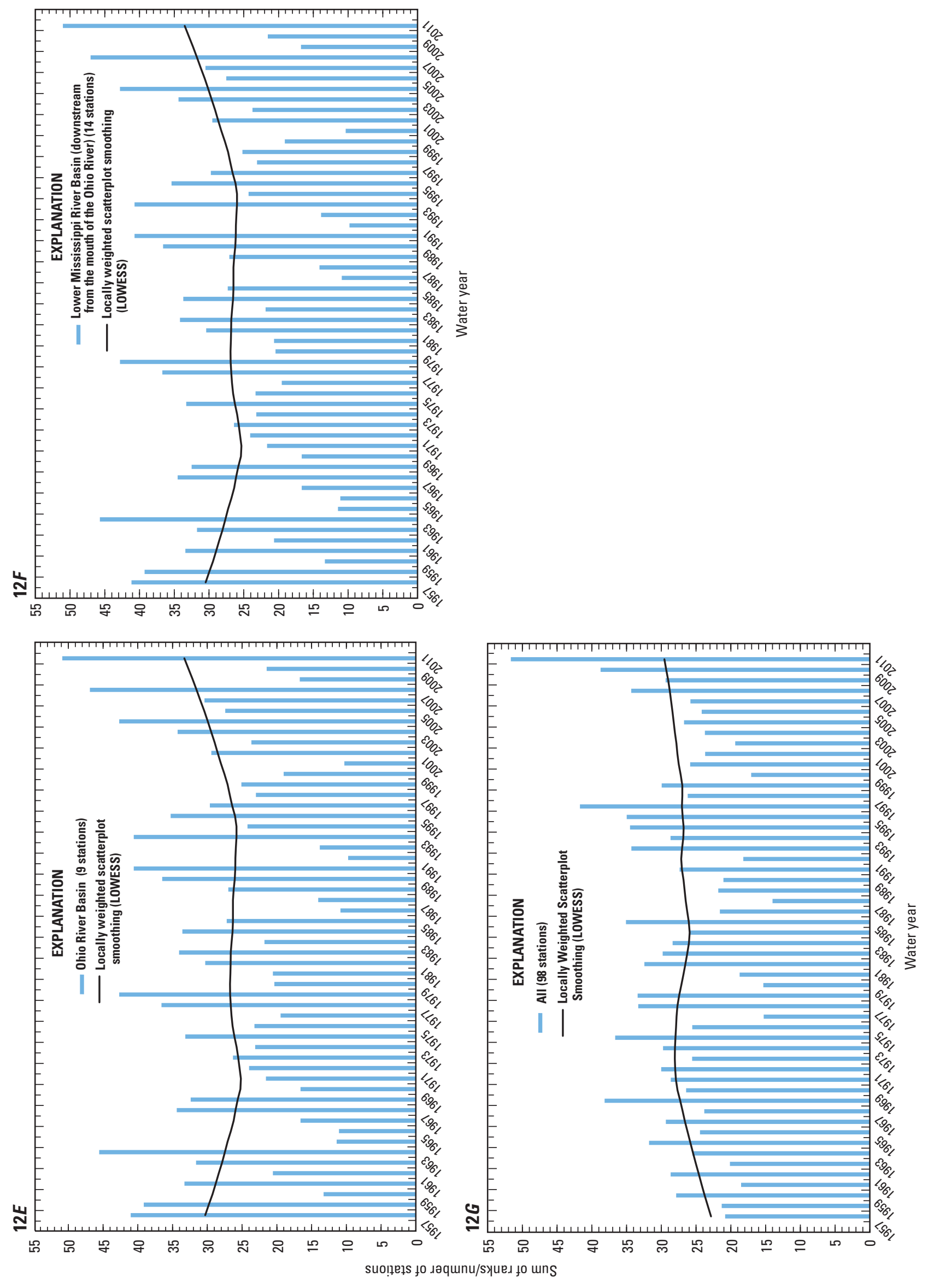

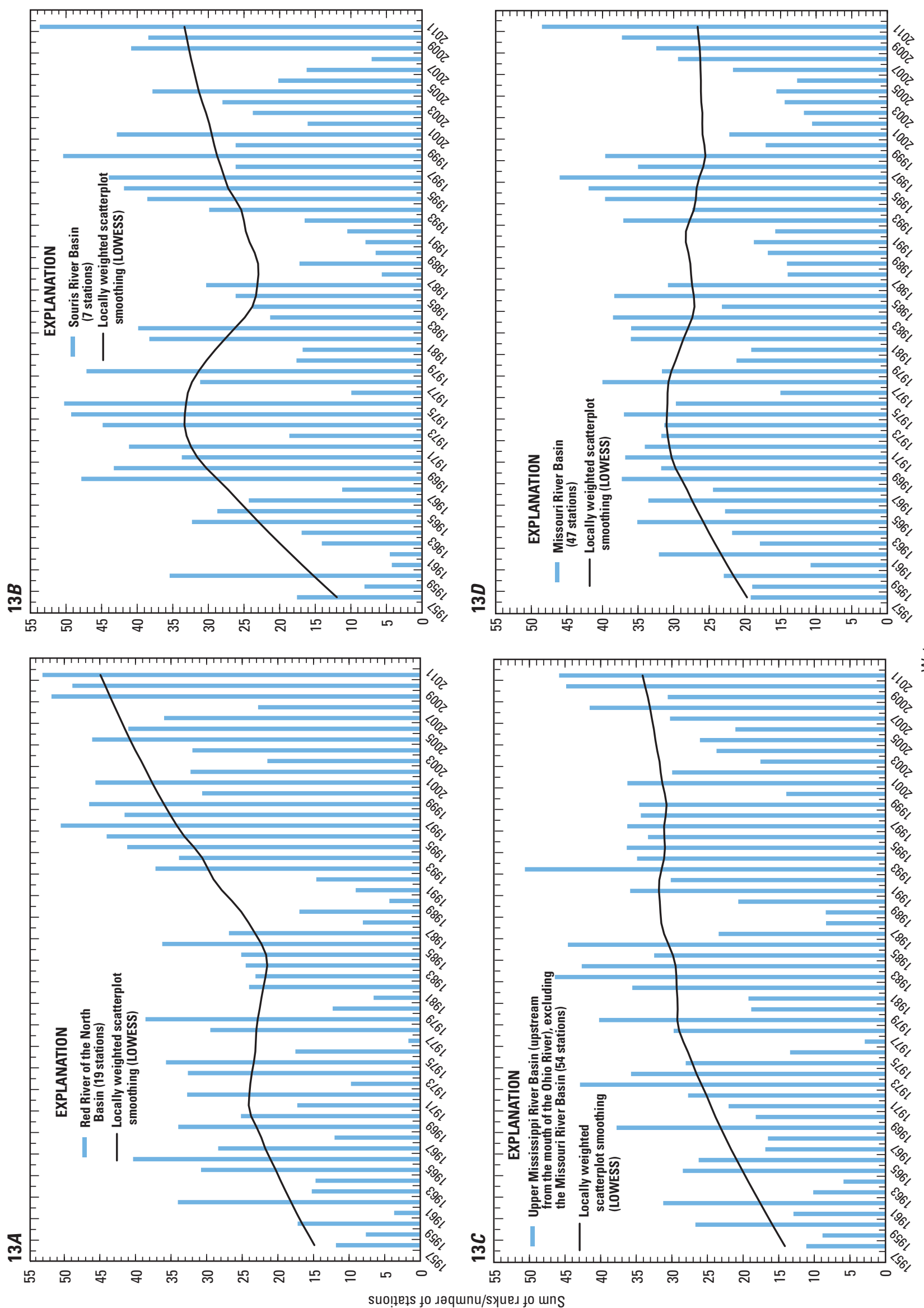

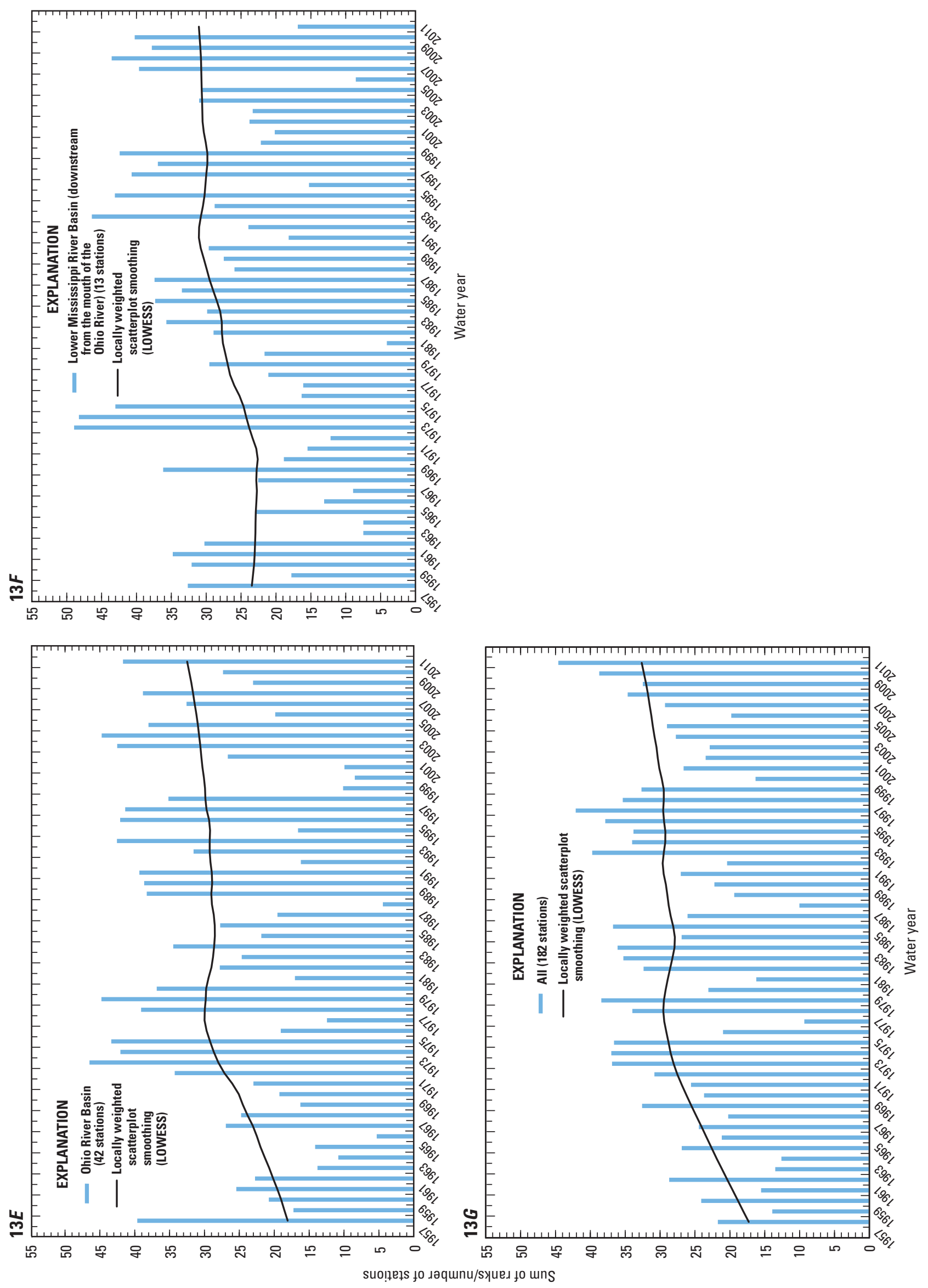


\section{Summary}

During 2011, flooding on numerous rivers in the Central United States, spanning from the Canadian border to the Gulf of Mexico and the Rocky Mountains to the Allegheny Mountains, resulted in 33 fatalities and approximately $\$ 4.2$ billion in damages. The flooding began in late February 2011 in parts of the Mississippi River Basin and continued in parts of the Mississippi River Basin or Souris and Red River of the North Basins until September 2011. The 2011 floods in the Central United States were caused by one or more combinations of the following: saturated antecedent soil conditions, higher than average streamflow at the beginning of 2011, rapid melting of the larger than normal snowpack, excess precipitation on snowpack, or simply excess precipitation. In some parts of the Central United States, precipitation amounts in excess of 20 inches in a 2-week period (700- to 1,000-percent above normal) were observed. This report provides broadscale characterizations of annual exceedance probabilities and trends for peak streamflows and annual runoff volumes for selected streamgages in the Central United States in areas affected by 2011 flooding.

Annual exceedance probabilities (AEPs) were analyzed for 321 streamgages for annual peak streamflow and for 211 streamgages for annual runoff volume. Within the Red River of the North Basin, AEPs for 2011 peak streamflows were not necessarily exceptional. However, AEPs for 2011 runoff volumes were exceptional, with 2 of 20 streamgages considered having AEPs less than 0.2 percent, 5 streamgages in the range of 0.2 to 1 percent, and 4 streamgages in the range of 1 to 2 percent. Magnitudes of 2011 runoff volumes also were exceptional, with all 11 of these streamgages eclipsing previous long-term (62 to 110 years) annual maxima by about one-third or more.

Flooding in the Souris River Basin was exceptional from all standpoints. Of 11 streamgages considered for AEP analysis of peak streamflows, 2011 flood peaks exceeded the next largest peak of record by at least double for 6 of the longestterm streamgages ( 75 to 108 years of peak-flow record). AEPs for these six streamgages were less than 1 percent. AEPs for 2011 runoff volumes were less than 1 percent for all seven streamgages considered for analysis. Magnitudes of annual runoff volumes also were exceptional, with all seven streamgages exceeding previous maxima (record lengths 52 to 108 years), and five of the seven streamgages exceeding by double or more.

AEPs for peak streamflows in the upper Mississippi River Basin were not exceptional, with no AEPs less than 1 percent. AEPs for annual runoff volumes indicated somewhat less frequent recurrence, with 11 having AEPs of less than 1 percent (out of 60 streamgages considered). The 2011 runoff volume for streamgage 05331000 (at Saint Paul, Minn.) exceeded the previous record (112 years of record) by about 24 percent.

An especially newsworthy feature was the prolonged flooding during 2011 along the main stem of the Missouri River downstream from Garrison Dam and extending downstream throughout the length of the Missouri River. The 2011 runoff volume for streamgage 06342500 (at Bismarck, North Dakota) exceeded the previous (1975) maximum by about 50 percent, with an associated AEP in the range of 0.2 to 1 percent.

Within the Ohio River Basin, most annual streamflow peaks occurred during early March to early May, with associated AEPs and runoff-volume AEPs that were not exceptional. Throughout the basin, peak-streamflow AEPs were less than 2 percent for only 4 streamgages (out of 25 considered), and runoff-volume AEPs were less than 2 percent for only 3 streamgages (out of 53 considered).

Along the lower Mississippi River, the largest streamflow peak in 91 years was recorded for streamgage 07289000 (at Vicksburg, Miss.), with an associated AEP of 0.8 percent. Of 55 streamgages considered for AEP analysis for peak streamflows, 10 had AEPs less than 1 percent. AEPs for annual runoff volume were larger than 4 percent for all lower Mississippi River streamgages considered, which resulted from extensive drought conditions in areas to the west.

Trends in peak streamflow were analyzed for 98 streamgages, with 67 streamgages having upward trends, 31 with downward trends, and zero with no trend. Trends in annual runoff volume were analyzed for 182 streamgages, with 145 streamgages having upward trends, 36 with downward trends, and 1 with no trend. The trend analyses used descriptive methods that did not include measures of statistical significance. A dichotomous spatial distribution in trends was apparent for both peak streamflow and annual runoff volume, with a small number of streamgages in the northwestern part of the study area having downward trends and most streamgages in the eastern part of the study area having upward trends.

\section{References Cited}

Anderson, M.T., Stamm, J.F., and Norton, P.A., 2008, Changes in monthly streamflow conditions in the Missouri River Basin from 1957 to 2007: Eos Transactions, American Geophysical Union, v. 89, no. 53, Fall Meeting Supplement, December 15-19, 2008, San Francisco, Calif., accessed March 14, 2013, at http://www.agu.org/meetings/fm08/ waisfm08.html.

Chin, E.H., Skelton, John, and Guy, H.P., 1975, The 1973 Mississippi River basin flood-Compilation and analysis of meteorologic, streamflow, and sediment data: U.S. Geological Survey Professional Paper 937, 137 p., accessed April 11, 2013, at http://pubs.er.usgs.gov/publication/pp937.

Cohn, T.A., Lane, W.L., and Stedinger, J.R., 2001, Confidence intervals for expected moments algorithm flood quantile estimates: Water Resources Research, v. 37, no. 6, p. 1,695-1,706, accessed May 15, 2013, at http:// dx.doi.org/10.1029/2001WR900016. 
Cohn, T.A., and Lins, H.F., 2005, Nature's style-Naturally trendy: Geophysical Research Letters, v. 32, L23402, 5 p., accessed April 12, 2013, at http://dx.doi. org/10.1029/2005GL024476.

Griffis, V.A., Stedinger, J.R., and Cohn, T.A., 2004, Log Pearson type 3 quantile estimators with regional skew information and low outlier adjustments: Water Resources Research, v. 40, W07503, 17 p., accessed May 10, 2013, at http://dx.doi.org/10.1029/2003WR002697.

Helsel, D.R., and Hirsch, R.M., 2002, Statistical methods in water resources techniques of water resources investigations, book 4, chap. A3, U.S. Geological Survey, 522 p., accessed April 12, 2013, at http://pubs.usgs.gov/ twri/twri4a3/.

Hirsch, R.M., 2011, A Perspective on nonstationarity and water management: Journal of the American Water Resources Association, v. 47, no. 3, p. 436-446, accessed April 23, 2013, at http://dx.doi.org/10.1111/j.17521688.2011.00539.x.

Hodgkins, G.A., Dudley, R.W., and Aichele, S.S., 2007, Historical changes in precipitation and streamflow in the U.S. Great Lakes Basin, 1915-2004: U.S. Geological Survey Scientific Investigations Report 2007-5118, 31 p., accessed April 12, 2013, at http://pubs.usgs.gov/sir/2007/5118/pdf/ SIR2007-5118.pdf.

Holmes, Jr., R.R., Wiche, G.J., Koenig, T.A., and Sando, S.K., 2013, Peak streamflows and runoff volumes for the Central United States, February through September, 2011: U.S. Geological Survey Professional Paper 1798-C, 60 p., accessed May 23, 2013, at http://pubs.usgs.gov/pp/1798c/.

Holmes, R.R., Jr., and Dinicola, Karen, 2010, 100-year flood-It's all about chance: U.S. Geological Survey General Information Product, $4 \mathrm{p}$.

Holmes, R.R., Jr., Koenig, T.A., and Karstensen, K.A., 2010, Flooding in the United States Midwest, 2008: U.S. Geological Survey Professional Paper 1775, 64 p., accessed April 11, 2013, at http://pubs.usgs.gov/pp/1775/.

Holmes, R.R., Jr., and Kupka, A.L., 1997, Floods of July 18-20, 1996, in northern Illinois: U.S. Geological Survey Open-File Report 97-425, 29 p., accessed April 11, 2013, at http://pubs.er.usgs.gov/publication/ofr97425.

Jennings, M.E., Thomas, T.O., Jr., and Riggs, H.C., 1994, Nationwide summary of U.S. Geological Survey regional regression equations estimating magnitude and frequency of floods for ungaged sites: U.S. Geological Survey WaterResources Investigations Report 94-4002, 196 p., accessed April 11, 2013, at http://pubs.er.usgs.gov/publication/ wri944002.
Mississippi River Commission, 1954, Annual highest and lowest stages of the Mississippi River and its outlets and tributaries to 1953: Vicksburg, Mississippi, 253 p.

Mississippi River Commission, 1955, Annual maximum minimum and mean discharges of the Mississippi River and its outlets and tributaries to 1953: Vicksburg, Mississippi, $140 \mathrm{p}$.

National Oceanic and Atmospheric Administration, 2011, United States flood loss report-Water year 2011: National Oceanic and Atmospheric Administration, National Weather Service, accessed April 11, 2013, at http://www.nws.noaa. gov/hic/summaries/WY2011.pdf.

National Oceanic and Atmospheric Administration, 2012a, Service assessment Spring 2011 Middle and Lower Mississippi River Valley Floods: National Oceanic and Atmospheric Administration National Weather Service Report of March 2012, 54 p., accessed on April 11, 2013, at http://www.nws.noaa.gov/om/assessments/pdfs/ MisssissippiRiverFloods12.pdf.

National Oceanic and Atmospheric Administration, 2012b, Service assessment of the Missouri/Souris River floods of May-August, 2011: National Oceanic and Atmospheric Administration National Weather Service Report of May 2012, 68 p., accessed on April 11, 2013, at http://www.nws. noaa.gov/om/assessments/pdfs/Missouri_floods11.pdf.

Nichols, Neville, 2001, The insignificance of significance testing: Bulletin of American Meteorology Society, v. 81, no. 5, p. 981-986, accessed April 12, 2013 at http://journals.ametsoc.org/doi/abs/10.1175/15200477\%282001\%29082\%3C0981\%3ACAATIO\%3E2.3.CO $\% 3 B 2$.

Niehus, C.A., Vecchia, A.V., Thompson, R.F., 1999a, Lakelevel frequency analysis for the Waubay Lakes Chain, northeastern South Dakota: U.S. Geological Survey WaterResources Investigation Report 99-4122, 166 p., accessed April 24, 2013, at, http://pubs.usgs.gov/wri/1999/4122/ report.pdf.

Niehus, C.A., Vecchia, A.V., and Thompson, R.F., 1999b, Supplement to Water-Resources Investigation Report 99-4122, Lake-level frequency analysis for the Waubay Lakes Chain, northeastern South Dakota: U.S. Geological Survey WaterResources Investigation Report 99-4251, 24 p., accessed April 24, 2013, at, http://pubs.usgs.gov/wri/1999/4251/ report.pdf.

North Dakota State Water Commission, 2013, Devils Lake flood facts: North Dakota State Water Commission Fact Sheet, March 2013, 4 p., accessed April 24, 2013, at http:// www.swc.state.nd.us/4DLink9/4dcgi/GetContentPDF/ PB-206/DL_Quick_Facts.pdf. 
Parrett, Charles, Melcher, Nick B., and James, Jr., Robert W., 1993, Flood Discharges in the Upper Mississippi River Basin, 1993, U.S. Geological Survey Circular 1120-A, 14 p., accessed April 11, 2103, at http://pubs.er.usgs.gov/ publication/cir1120A.

Parrett, Charles, and Johnson, D.R., 2003, Peak-flow frequency estimates based on data through water year 2001 for selected streamflow-gaging stations in South Dakota: U.S. Geological Water-Resources Investigations Report 20034308, 34 p., accessed April 11, 2013, at http://pubs.er.usgs. gov/publication/wri034308.

Salmi, T., Määttä, A., Anttila, P., Ruoho-Airola, T., and Amnell, T., 2002, Detecting trends of annual values of atmospheric pollutants by the Mann-Kendall test and Sen's slope estimates - The excel template application MAKESENS: Helsinki, Finland, Finnish Meteorological Institute Report 31, 35 p., accessed April 12, 2013, at http:// en.ilmatieteenlaitos. fi/makesens.

Sando, S.K., Driscoll, D.G., and Parrett, Charles, 2008, Peak-flow frequency estimates based on data through water year 2001 for selected streamflow-gaging stations in South Dakota: U.S. Geological Survey Scientific Investigations Report 2008-5104, 367 p., accessed April 11, 2013, at http://pubs.usgs.gov/sir/2008/5104/.

Sen, P.K., 1968, Estimates of the regression coefficient based on Kendall's tau: Journal of American Statistical Association, v. 63, p. 1,379-1,389, accessed April 13, 2013, at http://www.jstor.org/discover/10.2307/2285891?uid $=40407 \&$ uid $=3739784 \&$ uid $=2129 \&$ uid $=2 \&$ uid $=70 \&$ uid $=3 \&$ uid $=67 \&$ uid $=62 \&$ uid $=3739256 \&$ uid $=40401 \&$ sid $=21101894506203$.

South Dakota Department of Environment and Natural Resources, 2013, Historical lake levels in South Dakota, accessed April 24, 2013, at http://denr.sd.gov/des/wr/ dblakesearch.aspx.

U.S. Army Corps of Engineers, 2001, Final hydrology report-Hydrologic analyses, the Red River of the North main stem, Wahpeton/Breckenridge to Emerson, Manitoba: St. Paul, Minn.,149 p. plus attachments.

U.S. Army Corps of Engineers, 2004, Upper Mississippi River System Flow Frequency Study Final Report, Rock Island, Illinois: 40 p. plus appendices, accessed on April 17, 2013, at http://www.mvr.usace.army.mil/Missions/ FloodRiskManagement/UpperMississippiFlowFrequencySt udy.aspx.

U.S. Army Corps of Engineers, 2006, Missouri River Missouri River mainstem reservoir system master water control manual Missouri River Basin: 432 p., accessed on November 30, 2012, at http://www.nwd-mr.usace.army.mil/rcc/ reports/mmanual/MasterManual.pdf.
U.S. Army Corps of Engineers, 2010, Des Moines River Regulated Flow Frequency Study, Rock Island, Illinois: 95 p., accessed on April 17, 2013, at http://www.mvr.usace. army.mil/Portals/48/docs/FRM/DMRRFFS/DMRRFFSFinalReport.pdf.

U.S. Army Corps of Engineers, 2012, Summary of Engineering Data-Missouri River Main Stem System, accessed June 6, 2012, at http://www.nwd-mr.usace.army.mil/rcc/ projdata/summaryengdat.pdf.

U.S. Geological Survey, 2013, National Water Information System-USGS water data for the nation: U.S. Geological Survey Web interface, accessed on May 10, 2013, at http:// waterdata.usgs.gov/nwis.

U.S. Interagency Advisory Committee on Water Data, 1982, Guidelines for determining flood flow frequency: Hydrology Subcommittee, Bulletin 17B, appendices 1-14, 28 p.

Vecchia, A.V., 2008, Simulation of the effects of Devils Lake outlet alternatives on future lake levels and water quality in the Sheyenne River and Red River of the North: U.S. Geological Survey Scientific Investigations Report 2011-5050, 60 p., accessed on April 29, 2013, at http://pubs.usgs.gov/ sir/2011/5050/.

Vining, K.C., Chase, K.J., and Loss, G.R., 2013, General weather conditions and precipitation contributing to the 2011 flooding in the Mississippi and Red River of the North Basins, December 2010 through July 2011: U.S. Geological Survey Professional Paper 1798-B, 22 p., accessed on April 11, 2013, at http://pubs.er.usgs.gov/publication/ pp1798B.

Watermolen, John, 2005, 1:2,000,000-Scale Hydrologic Unit Boundaries: National Atlas of the United States, Reston, Va. (Also available at http://nationalatlas.gov/mld/hucs00m. html.)

Westerman, D.A., Merriman, K.R., De Lanois, J.L., and Berenbrock, Charles, 2013, Analysis and inundation mapping of the April-May 2011 flood at selected locations in northern and eastern Arkansas and southern Missouri: U.S. Geological Survey Scientific Investigations Report 20135148, 44 p., accessed on October 31, 2013, at http://pubs. usgs.gov/sir/2013/5148/. 


\section{Tables 1 and 2}




\section{Annual Exceedance Probabilities and Trends for the Central United States during the 2011 Floods}

Table 1. Peak streamflow data and results of flood-probability analyses for selected streamgages in the Central United States affected by 2011 flooding.

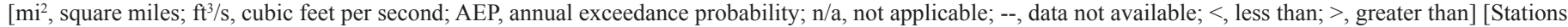
used in the trend analysis are italicized; Stations used in the trend analysis that overlap with trend analyses of Holmes and others (2010) are italicized and bold]

\section{Flood data}

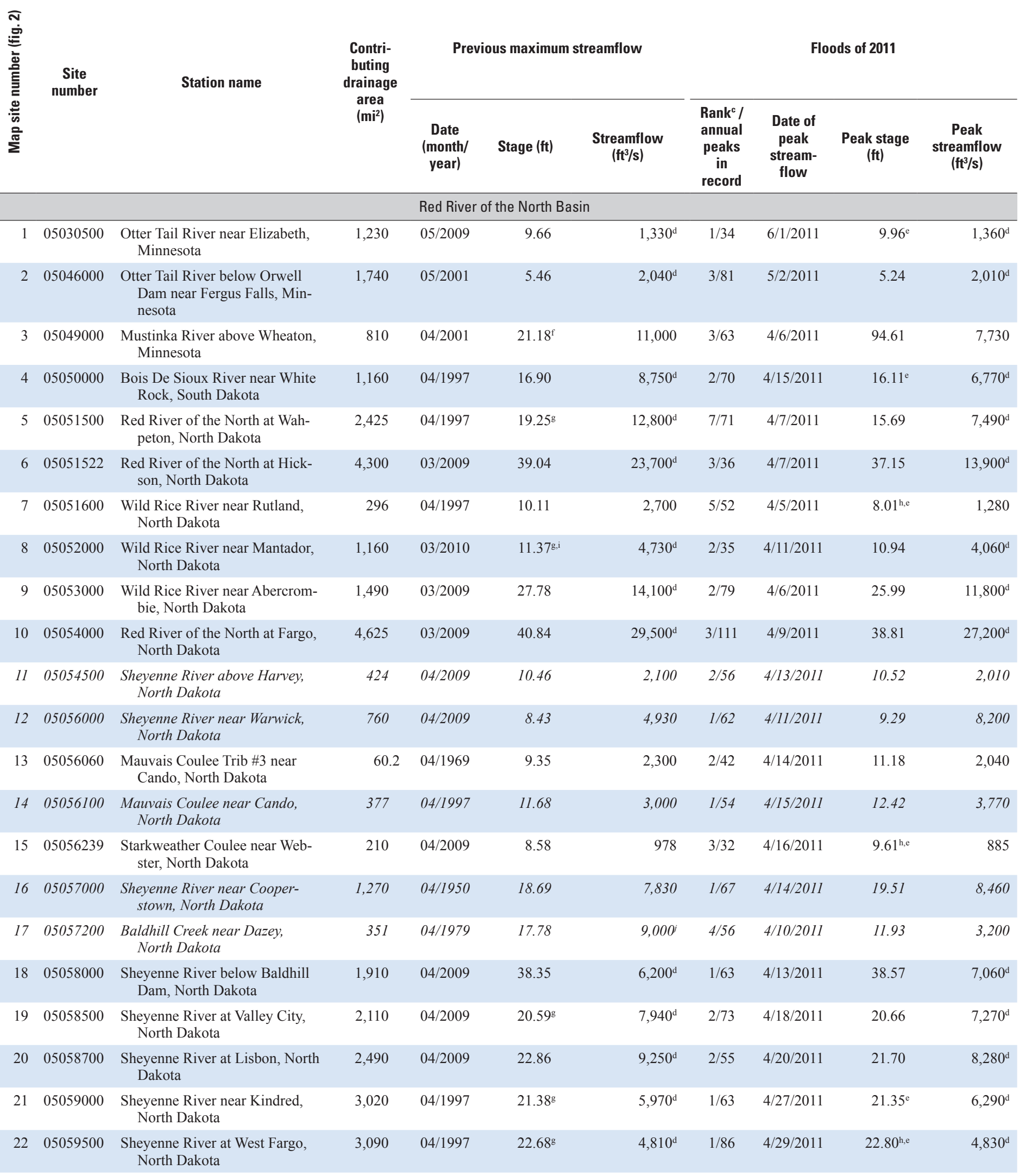




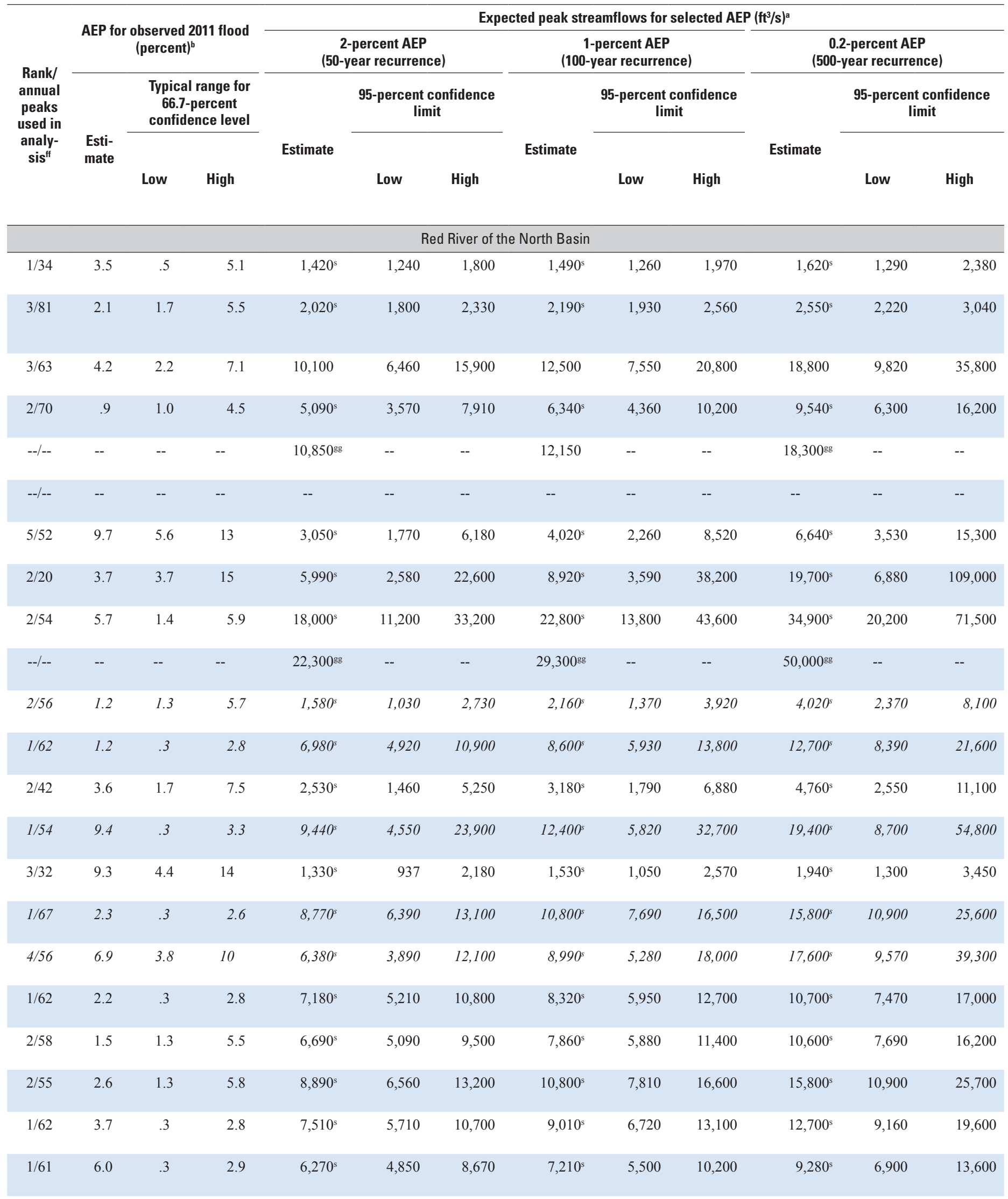




\section{Annual Exceedance Probabilities and Trends for the Central United States during the 2011 Floods}

Table 1. Peak streamflow data and results of flood-probability analyses for selected streamgages in the Central United States affected by 2011 flooding.-Continued

$\left[\mathrm{mi}^{2}\right.$, square miles; $\mathrm{ft}^{3} / \mathrm{s}$, cubic feet per second; AEP, annual exceedance probability; n/a, not applicable; --, data not available; <, less than; >, greater than] [Stations used in the trend analysis are italicized; Stations used in the trend analysis that overlap with trend analyses of Holmes and others (2010) are italicized and bold]

Flood data

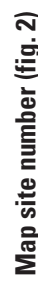

Contributing

Site number

Station name

drainage

area
$\left(\mathrm{mi}^{2}\right)$
Previous maximum streamflow

Floods of 2011

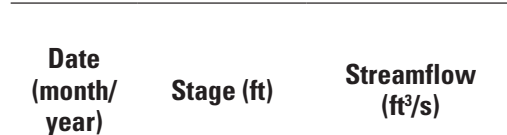

\begin{tabular}{|c|c|c|c|}
\hline $\begin{array}{l}\text { Rank }^{c} / \\
\text { annual } \\
\text { peaks } \\
\text { in }\end{array}$ & $\begin{array}{l}\text { Date of } \\
\text { peak } \\
\text { stream- } \\
\text { flow }\end{array}$ & $\begin{array}{c}\text { Peak stage } \\
\text { (ft) }\end{array}$ & $\begin{array}{c}\text { Peak } \\
\text { streamflow } \\
\left(\mathrm{ft}^{3} / \mathrm{s}\right)\end{array}$ \\
\hline
\end{tabular}

\begin{tabular}{|c|c|c|c|c|c|c|c|c|c|c|}
\hline \multicolumn{11}{|c|}{ Red River of the North Basin-Continued } \\
\hline 23 & 05059600 & $\begin{array}{l}\text { Maple River near Hope, North } \\
\text { Dakota }\end{array}$ & 17.4 & $03 / 2004$ & $6.98^{\mathrm{g}}$ & 1,000 & $1 / 47$ & $8 / 1 / 2011$ & 8.05 & 1,340 \\
\hline 24 & 05059700 & $\begin{array}{l}\text { Maple River near Enderlin, } \\
\quad \text { North Dakota }\end{array}$ & 796 & $06 / 1975$ & 15.41 & 7,610 & $3 / 56$ & $4 / 11 / 2011$ & 13.31 & 6,830 \\
\hline 25 & 05060400 & $\begin{array}{l}\text { Sheyenne River at Harwood, } \\
\text { North Dakota }\end{array}$ & $--^{k}$ & 04/1997 & 92.02 & $11,000^{\mathrm{j}, \mathrm{d}}$ & $1 / 16$ & $4 / 10 / 2011$ & 91.82 & $13,000^{\mathrm{d}}$ \\
\hline 26 & 05060500 & $\begin{array}{l}\text { Rush River at Amenia, North } \\
\text { Dakota }\end{array}$ & 116 & 04/1979 & $10.37^{g}$ & 3,490 & $5 / 65$ & $4 / 7 / 2011$ & 11.00 & 1,970 \\
\hline 29 & 05064500 & $\begin{array}{l}\text { Red River of the North at Hals- } \\
\text { tad, Minnesota }\end{array}$ & 15,205 & $04 / 1997$ & 40.74 & $71,500^{\mathrm{d}}$ & $3 / 72$ & $4 / 12 / 2011$ & 40.51 & $60,700^{\mathrm{d}}$ \\
\hline 30 & 05065500 & $\begin{array}{l}\text { Goose River near Portland, } \\
\text { North Dakota }\end{array}$ & 407 & $05 / 1950$ & $27.10^{\mathrm{f}}$ & 8,530 & $6 / 46$ & 4/8/2011 & 25.15 & 3,220 \\
\hline 31 & 05066500 & $\begin{array}{l}\text { Goose River at Hillsboro, North } \\
\text { Dakota }\end{array}$ & 1,093 & 04/1979 & 16.76 & 14,800 & $2 / 85$ & 4/9/2011 & 15.50 & 10,000 \\
\hline 32 & 05069000 & $\begin{array}{l}\text { Sand Hill River at Climax, Min- } \\
\quad \text { nesota }\end{array}$ & 420 & $04 / 1965$ & 17.81 & 4,560 & $1 / 69$ & $4 / 10 / 2011$ & $34.56^{h, e}$ & $4,800^{j, l}$ \\
\hline 33 & 05070000 & $\begin{array}{l}\text { Red River of the North near } \\
\text { Thompson, North Dakota }\end{array}$ & 24,010 & $04 / 2009$ & 64.56 & $61,300^{\mathrm{d}}$ & $1 / 13$ & $4 / 13 / 2011$ & 65.18 & $72,000^{\mathrm{d}}$ \\
\hline 34 & 05076000 & $\begin{array}{l}\text { Thief River near Thief River } \\
\text { Falls, Minnesota }\end{array}$ & 985 & $05 / 1950$ & 17.38 & 5,610 & $3 / 100$ & $4 / 6 / 2011$ & 16.07 & 4,640 \\
\hline 36 & 05082500 & $\begin{array}{l}\text { Red River of the North at Grand } \\
\text { Forks, North Dakota }\end{array}$ & 21,445 & 04/1997 & $52.04^{\mathrm{g}}$ & $137,000^{\mathrm{d}}$ & $2 / 130$ & $4 / 14 / 2011$ & 49.86 & $87,500^{\mathrm{d}}$ \\
\hline 37 & 05083500 & $\begin{array}{l}\text { Red River of the North at Oslo, } \\
\text { Minnesota }\end{array}$ & 22,520 & 04/1997 & 38.00 & $120,000^{\mathrm{d}}$ & $2 / 49$ & $4 / 14 / 2011$ & 38.09 & $81,400^{\mathrm{d}}$ \\
\hline 41 & 05092000 & $\begin{array}{l}\text { Red River of the North at Dray- } \\
\text { ton, North Dakota }\end{array}$ & 26,085 & 04/1997 & 45.55 & $124,000^{\mathrm{d}}$ & $5 / 74$ & $4 / 19 / 2011$ & 43.17 & $83,000^{\mathrm{d}}$ \\
\hline 43 & 05099600 & $\begin{array}{l}\text { Pembina River at Walhalla, } \\
\text { North Dakota }\end{array}$ & 3,350 & 04/1997 & 16.53 & $23,100^{\mathrm{m}}$ & $4 / 65$ & 4/18/2011 & 16.55 & 16,300 \\
\hline 44 & 05100000 & $\begin{array}{l}\text { Pembina River at Neche, North } \\
\text { Dakota }\end{array}$ & 3,410 & $04 / 2009$ & 21.61 & 16,900 & $2 / 104$ & $4 / 19 / 2011$ & $21.78^{h, e}$ & 15,300 \\
\hline \multicolumn{11}{|c|}{ Souris River Basin } \\
\hline 46 & 05113600 & $\begin{array}{l}\text { Long Creek near Noonan, North } \\
\text { Dakota }\end{array}$ & 630 & 03/1976 & 17.61 & 6,310 & $1 / 52$ & $6 / 21 / 2011$ & 19.55 & 10,800 \\
\hline 47 & 05114000 & $\begin{array}{l}\text { Souris River near Sherwood, } \\
\text { North Dakota }\end{array}$ & 3,040 & 04/1976 & 25.15 & $14,800^{\mathrm{d}}$ & $1 / 82$ & 6/23/2011 & 28.16 & $29,700^{\mathrm{d}}$ \\
\hline 48 & 05116000 & $\begin{array}{l}\text { Souris River near Foxholm, } \\
\text { North Dakota }\end{array}$ & 3,270 & $04 / 1976$ & 17.17 & $8,600^{\mathrm{d}}$ & $1 / 75$ & $6 / 25 / 2011$ & 22.44 & $26,400^{\mathrm{d}}$ \\
\hline 49 & 05116500 & $\begin{array}{l}\text { Des Lacs River at Foxholm, } \\
\text { North Dakota }\end{array}$ & 539 & 04/1979 & 21.23 & $4,260^{\mathrm{d}}$ & $3 / 66$ & $6 / 1 / 2011$ & 20.57 & $3,620^{\mathrm{d}}$ \\
\hline 50 & 05117500 & $\begin{array}{l}\text { Souris River above Minot, North } \\
\text { Dakota }\end{array}$ & 3,900 & 04/1904 & $21.90^{\mathrm{fj}}$ & $12,000^{\mathrm{m}}$ & $1 / 108$ & 6/25/2011 & 24.37 & $26,900^{\mathrm{d}}$ \\
\hline 51 & 05120000 & $\begin{array}{l}\text { Souris River near Verendrye, } \\
\text { North Dakota }\end{array}$ & 4,400 & $04 / 2009$ & 17.92 & $10,900^{\mathrm{d}}$ & $1 / 75$ & $6 / 26 / 2011$ & 18.53 & $26,900^{\mathrm{d}}$ \\
\hline 52 & 05120500 & $\begin{array}{l}\text { Wintering River near Karlsruhe, } \\
\text { North Dakota }\end{array}$ & 285 & 04/1949 & $12.00^{\mathrm{h}}$ & $3,000^{\mathrm{d}}$ & $6 / 75$ & $4 / 12 / 2011$ & $8.87^{\mathrm{h}, \mathrm{e}}$ & $1,360^{\mathrm{d}}$ \\
\hline
\end{tabular}




\begin{tabular}{|c|c|c|c|c|c|c|c|c|c|c|c|c|}
\hline \multirow{4}{*}{$\begin{array}{c}\text { Rank/ } \\
\text { annual } \\
\text { peaks } \\
\text { used in } \\
\text { analy- } \\
\text { sis }^{f f}\end{array}$} & \multirow{2}{*}{\multicolumn{3}{|c|}{$\begin{array}{l}\text { AEP for observed } 2011 \text { flood } \\
\text { (percent) }\end{array}$}} & \multicolumn{9}{|c|}{ Expected peak streamflows for selected AEP $\left(\mathrm{ft}^{3} / \mathrm{s}\right)^{\mathrm{a}}$} \\
\hline & & & & \multicolumn{3}{|c|}{$\begin{array}{c}\text { 2-percent AEP } \\
\text { (50-year recurrence) }\end{array}$} & \multicolumn{3}{|c|}{$\begin{array}{c}\text { 1-percent AEP } \\
\text { (100-year recurrence) }\end{array}$} & \multicolumn{3}{|c|}{$\begin{array}{c}\text { 0.2-percent AEP } \\
\text { (500-year recurrence) }\end{array}$} \\
\hline & \multirow{2}{*}{$\begin{array}{l}\text { Esti- } \\
\text { mate }\end{array}$} & \multicolumn{2}{|c|}{$\begin{array}{c}\text { Typical range for } \\
66.7-\text { percent } \\
\text { confidence level }\end{array}$} & \multirow{2}{*}{ Estimate } & \multicolumn{2}{|c|}{$\begin{array}{l}\text { 95-percent confidence } \\
\text { limit }\end{array}$} & \multirow{2}{*}{ Estimate } & \multicolumn{2}{|c|}{$\begin{array}{l}\text { 95-percent confidence } \\
\text { limit }\end{array}$} & \multirow{2}{*}{ Estimate } & \multicolumn{2}{|c|}{$\begin{array}{l}\text { 95-percent confidence } \\
\text { limit }\end{array}$} \\
\hline & & Low & High & & Low & High & & Low & High & & Low & High \\
\hline \multicolumn{13}{|c|}{ Red River of the North Basin-Continued } \\
\hline $1 / 47$ & 3.4 & .4 & 3.7 & $1,550^{\mathrm{s}}$ & 1,010 & 2,700 & $1,850^{\mathrm{s}}$ & 1,190 & 3,320 & $2,530^{\mathrm{s}}$ & 1,570 & 4,780 \\
\hline $3 / 56$ & 6.3 & 2.5 & 8.0 & $10,900^{s}$ & 6,920 & 19,500 & $13,900^{s}$ & 8,580 & 25,800 & $21,400^{s}$ & 12,600 & 42,600 \\
\hline $1 / 16$ & 9.5 & 1.1 & 11 & $17,800^{\mathrm{s}}$ & 12,400 & 31,700 & $19,600^{\mathrm{s}}$ & 13,500 & 36,300 & $23,400^{\mathrm{s}}$ & 15,600 & 46,200 \\
\hline $5 / 65$ & 8.5 & 4.5 & 11 & $3,540^{s}$ & 2,460 & 5,620 & $4,420^{s}$ & 3,000 & 7,230 & $6,650^{s}$ & 4,330 & 11,600 \\
\hline --/-- & -- & -- & -- & $50,700^{\mathrm{gg}}$ & 40,500 & 66,300 & $62,200^{\mathrm{gg}}$ & 48,900 & 83,300 & $93,000^{\mathrm{gg}}$ & 70,600 & 130,000 \\
\hline $6 / 46$ & 9.2 & 8.1 & 17 & $6,470^{\mathrm{s}}$ & 4,240 & 11,300 & $8,330^{\mathrm{s}}$ & 5,310 & 15,200 & $13,600^{\mathrm{s}}$ & 8,160 & 27,200 \\
\hline $2 / 81$ & 5.0 & .9 & 3.9 & $14,100^{s}$ & 9,730 & 22,100 & $17,500^{s}$ & 11,900 & 28,300 & $25,800^{s}$ & 16,900 & 43,700 \\
\hline $1 / 69$ & 4.9 & .3 & 2.6 & 6,170 & 4,480 & 8,500 & 7,330 & 5,080 & 10,600 & 10,100 & 6,270 & 16,200 \\
\hline$--/--$ & -- & -- & -- & -- & -- & -- & -- & -- & -- & -- & -- & -- \\
\hline $3 / 100$ & 3.7 & 1.4 & 4.5 & 5,070 & 3,910 & 6,570 & 5,490 & 4,050 & 7,460 & 6,260 & 4,180 & 9,360 \\
\hline$--/--$ & -- & -- & -- & $87,600^{\mathrm{gg}}$ & 71,000 & 113,000 & $108,000^{\mathrm{gg}}$ & 85,800 & 141,000 & $161,000^{\mathrm{gg}}$ & 124,000 & 220,000 \\
\hline --/-- & -- & -- & -- & -- & -- & -- & -- & -- & -- & -- & -- & -- \\
\hline --/-- & -- & -- & -- & $91,200^{\mathrm{gg}}$ & 70,600 & 126,000 & $112,000^{\mathrm{gg}}$ & 84,900 & 160,000 & $169,000^{\mathrm{gg}}$ & 123,000 & 258,000 \\
\hline $3 / 64$ & 4.0 & 2.2 & 7.0 & $21,600^{\mathrm{s}}$ & 15,000 & 34,200 & $27,800^{\mathrm{s}}$ & 18,900 & 45,700 & $45,300^{\mathrm{s}}$ & 29,200 & 80,200 \\
\hline $2 / 104$ & 1.9 & .7 & 3.1 & $15,100^{s}$ & 11,400 & 21,300 & $19,500^{s}$ & 14,300 & 28,200 & $31,700^{s}$ & 22,400 & 48,600 \\
\hline \multicolumn{13}{|c|}{ Souris River Basin } \\
\hline $1 / 52$ & 2.3 & .4 & 3.4 & $11,500^{s}$ & 6,390 & 24,600 & $15,600^{s}$ & 8,380 & 35,000 & $27,100^{s}$ & 13,700 & 66,700 \\
\hline $1 / 70$ & .5 & .3 & 2.5 & $14,200^{\mathrm{s}}$ & 9,540 & 23,400 & $19,500^{\mathrm{s}}$ & 12,700 & 33,500 & $36,500^{\mathrm{s}}$ & 22,300 & 68,800 \\
\hline $1 / 75$ & .4 & .2 & 2.4 & $9,220^{\mathrm{s}}$ & 6,230 & 15,100 & $14,000^{\mathrm{s}}$ & 9,040 & 24,200 & $33,300^{\mathrm{s}}$ & 19,700 & 65,500 \\
\hline $3 / 66$ & 3.1 & 2.1 & 6.8 & $4,200^{\mathrm{s}}$ & 2,840 & 6,870 & $5,250^{\mathrm{s}}$ & 3,470 & 8,860 & $7,880^{\mathrm{s}}$ & 5,000 & 14,100 \\
\hline $1 / 76$ & .2 & .2 & 2.3 & $9,490^{\mathrm{s}}$ & 6,740 & 14,600 & $13,400^{\mathrm{s}}$ & 9,220 & 21,600 & $27,600^{\mathrm{s}}$ & 17,600 & 49,200 \\
\hline $1 / 75$ & $<0.2$ & .2 & 2.4 & $10,500^{\mathrm{s}}$ & 7,630 & 15,700 & $14,200^{\mathrm{s}}$ & 10,000 & 22,200 & $26,500^{\mathrm{s}}$ & 17,500 & 45,100 \\
\hline $6 / 75$ & 5.2 & 5.0 & 11 & $2,220^{\mathrm{s}}$ & 1,460 & 3,760 & $3,090^{\mathrm{s}}$ & 1,970 & 5,460 & $5,880^{\mathrm{s}}$ & 3,510 & 11,300 \\
\hline
\end{tabular}




\section{Annual Exceedance Probabilities and Trends for the Central United States during the 2011 Floods}

Table 1. Peak streamflow data and results of flood-probability analyses for selected streamgages in the Central United States affected by 2011 flooding.-Continued

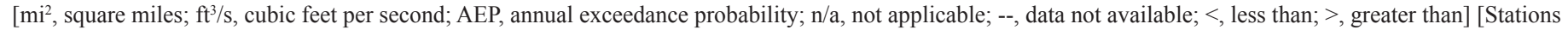
used in the trend analysis are italicized; Stations used in the trend analysis that overlap with trend analyses of Holmes and others (2010) are italicized and bold]

Flood data

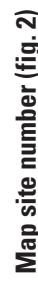

Contributing

Site number

Station name
Previous maximum streamflow

drainage

area
$\left(\mathrm{mi}^{2}\right)$
Floods of 2011

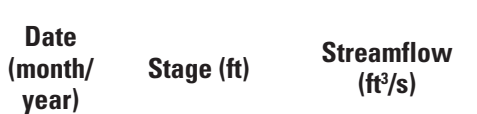

\begin{tabular}{cc}
\hline Rank $^{c} /$ & Date of \\
annual \\
peaks & $\begin{array}{c}\text { peak } \\
\text { in }\end{array}$ \\
stream- \\
flow
\end{tabular}

record

\begin{tabular}{|c|c|c|c|c|c|c|c|c|c|c|}
\hline \multicolumn{11}{|c|}{ Souris River Basin-Continued } \\
\hline 53 & 05122000 & $\begin{array}{l}\text { Souris River near Bantry, North } \\
\text { Dakota }\end{array}$ & 4,700 & $04 / 1976$ & 14.59 & $9,330^{\mathrm{d}}$ & $1 / 75$ & $6 / 28 / 2011$ & 16.90 & $30,000^{\mathrm{d}}$ \\
\hline 54 & 05123400 & $\begin{array}{l}\text { Willow Creek near Willow City, } \\
\text { North Dakota }\end{array}$ & 730 & $04 / 1969$ & 16.76 & 5,900 & $4 / 75$ & $4 / 12 / 2011$ & 15.24 & 2,900 \\
\hline 55 & 05123510 & $\begin{array}{l}\text { Deep River near Upham, North } \\
\text { Dakota }\end{array}$ & 370 & $04 / 1969$ & 18.18 & 6,760 & $2 / 49$ & $4 / 11 / 2011$ & 17.62 & 5,110 \\
\hline 56 & 05124000 & $\begin{array}{l}\text { Souris River near Westhope, } \\
\text { North Dakota }\end{array}$ & 6,600 & $04 / 1976$ & 19.16 & $12,600^{\mathrm{d}}$ & $1 / 82$ & $7 / 5 / 2011$ & $22.95^{\mathrm{e}}$ & $30,400^{\mathrm{d}}$ \\
\hline \multicolumn{11}{|c|}{ Upper Mississippi River Basin (upstream of the mouth of the Ohio River), excluding the Missouri River Basin } \\
\hline 61 & 05278930 & $\begin{array}{l}\text { Buffalo Creek near Glencoe, } \\
\text { Minnesota }\end{array}$ & 373 & 09/1991 & 11.78 & $4,300^{\mathrm{n}}$ & $4 / 27$ & $3 / 25 / 2011$ & $19.59^{\mathrm{h}, \mathrm{e}}$ & 3,490 \\
\hline 62 & 05280000 & $\begin{array}{l}\text { Crow River at Rockford, Min- } \\
\text { nesota }\end{array}$ & 2,640 & 04/1965 & 19.27 & 22,400 & 9/90 & $4 / 10 / 2011$ & 13.82 & 11,700 \\
\hline 64 & 05287890 & $\begin{array}{l}\text { Elm Creek near Champlin, Min- } \\
\text { nesota }\end{array}$ & 86.0 & $04 / 2001$ & 10.02 & 875 & $3 / 33$ & $3 / 24 / 2011$ & 9.81 & 803 \\
\hline 66 & 05288705 & $\begin{array}{l}\text { Shingle Creek at Queen Ave In } \\
\text { Minneapolis, Minnesota }\end{array}$ & 28.2 & $09 / 2005$ & 13.26 & $291^{\circ}$ & $1 / 15$ & 7/16/2011 & $13.93^{\mathrm{h}}$ & $301^{\circ}$ \\
\hline 67 & 05292000 & $\begin{array}{l}\text { Minnesota River at Ortonville, } \\
\text { Minnesota }\end{array}$ & 1,160 & $03 / 2010$ & 12.84 & $5,680^{d}$ & $5 / 74$ & $4 / 9 / 2011$ & 10.28 & $3,840^{\mathrm{d}}$ \\
\hline 68 & 05293371 & $\begin{array}{l}\text { Pomme De Terre River below } \\
\text { Elbow Lake, Minnesota }\end{array}$ & 340 & $03 / 2009$ & 6.81 & 744 & $1 / 26$ & $7 / 15 / 2011$ & 7.36 & 910 \\
\hline 69 & 05294000 & $\begin{array}{l}\text { Pomme De Terre River at Apple- } \\
\text { ton, Minnesota }\end{array}$ & 905 & $04 / 1997$ & 18.13 & $8,890^{p}$ & $7 / 79$ & $4 / 6 / 2011$ & 11.00 & 3,170 \\
\hline 71 & 05301000 & $\begin{array}{l}\text { Minnesota River near Lac Qui } \\
\text { Parle, Minnesota }\end{array}$ & 4,050 & 04/1997 & -- & $43,000^{\mathrm{i}, \mathrm{d}, \mathrm{m}}$ & $5 / 66$ & $4 / 6 / 2011$ & 38.30 & $19,700^{\mathrm{d}}$ \\
\hline 72 & 05311000 & $\begin{array}{l}\text { Minnesota River at Montevideo, } \\
\text { Minnesota }\end{array}$ & 6,180 & 04/1997 & 23.90 & $47,500^{\mathrm{d}}$ & $6 / 102$ & $4 / 7 / 2011$ & 19.76 & $23,700^{\mathrm{d}}$ \\
\hline 73 & 05313500 & $\begin{array}{l}\text { Yellow Medicine River near } \\
\text { Granite Falls, Minnesota }\end{array}$ & 664 & $04 / 1969$ & 14.90 & 17,200 & $8 / 80$ & $3 / 25 / 2011$ & 9.77 & 6,620 \\
\hline 74 & 05316500 & $\begin{array}{l}\text { Redwood River near Redwood } \\
\quad \text { Falls, Minnesota }\end{array}$ & 629 & $06 / 1957$ & 15.92 & 19,700 & $6 / 86$ & $3 / 25 / 2011$ & $15.51^{h, e}$ & $7,370^{j, l}$ \\
\hline 75 & 05316950 & $\begin{array}{l}\text { Cottonwood River near Spring- } \\
\text { field, Minnesota }\end{array}$ & 777 & 04/1969 & 31.55 & $18,300^{\mathrm{m}}$ & $4 / 40$ & $3 / 23 / 2011$ & 30.06 & 9,480 \\
\hline 76 & 05317000 & $\begin{array}{l}\text { Cottonwood River near New } \\
\text { Ulm, Minnesota }\end{array}$ & 1,300 & $04 / 1969$ & 19.15 & 28,700 & $6 / 85$ & $3 / 24 / 2011$ & 17.15 & 15,100 \\
\hline 77 & 05319500 & $\begin{array}{l}\text { Watonwan River near Garden } \\
\text { City, Minnesota }\end{array}$ & 851 & $04 / 1965$ & 18.89 & $19,000^{\mathrm{m}}$ & $6 / 44$ & $3 / 24 / 2011$ & 13.64 & 9,730 \\
\hline 78 & 05320000 & $\begin{array}{l}\text { Blue Earth River near Rapidan, } \\
\text { Minnesota }\end{array}$ & 2,410 & $04 / 1965$ & 21.36 & 43,100 & $7 / 99$ & $3 / 22 / 2011$ & 12.70 & $18,600^{\mathrm{d}}$ \\
\hline 79 & 05320270 & $\begin{array}{l}\text { Little Cobb River near Beauford, } \\
\text { Minnesota }\end{array}$ & 130 & $09 / 2010$ & 15.31 & 5,120 & $2 / 15$ & $3 / 20 / 2011$ & 13.62 & 3,380 \\
\hline 80 & 05320480 & $\begin{array}{l}\text { Maple River near Rapidan, Min- } \\
\text { nesota }\end{array}$ & 338 & $09 / 2010$ & 18.30 & 12,800 & $4 / 40$ & $3 / 20 / 2011$ & 13.09 & 4,660 \\
\hline 81 & 05320500 & $\begin{array}{l}\text { Le Sueur River near Rapidan, } \\
\text { Minnesota }\end{array}$ & 1,110 & $09 / 2010$ & 21.35 & 30,500 & $4 / 69$ & $3 / 21 / 2011$ & 12.45 & 14,200 \\
\hline
\end{tabular}




\begin{tabular}{|c|c|c|c|c|c|c|c|c|c|c|c|c|}
\hline \multirow{4}{*}{$\begin{array}{c}\text { Rank/ } \\
\text { annual } \\
\text { peaks } \\
\text { used in } \\
\text { analy- } \\
\text { sis }^{f \prime}\end{array}$} & \multirow{2}{*}{\multicolumn{3}{|c|}{$\begin{array}{l}\text { AEP for observed } 2011 \text { flood } \\
\text { (percent) })^{b}\end{array}$}} & \multicolumn{9}{|c|}{ Expected peak streamflows for selected AEP $\left(\mathrm{ft}^{3} / \mathrm{s}\right)^{\mathrm{a}}$} \\
\hline & & & & \multicolumn{3}{|c|}{$\begin{array}{c}\text { 2-percent AEP } \\
\text { (50-year recurrence) }\end{array}$} & \multicolumn{3}{|c|}{$\begin{array}{c}\text { 1-percent AEP } \\
\text { (100-year recurrence) }\end{array}$} & \multicolumn{3}{|c|}{$\begin{array}{c}\text { 0.2-percent AEP } \\
\text { (500-year recurrence) }\end{array}$} \\
\hline & \multirow{2}{*}{$\begin{array}{l}\text { Esti- } \\
\text { mate }\end{array}$} & \multicolumn{2}{|c|}{$\begin{array}{l}\text { Typical range for } \\
66.7-\text { percent } \\
\text { confidence level }\end{array}$} & \multirow{2}{*}{ Estimate } & \multicolumn{2}{|c|}{$\begin{array}{l}\text { 95-percent confidence } \\
\text { limit }\end{array}$} & \multirow{2}{*}{ Estimate } & \multicolumn{2}{|c|}{$\begin{array}{l}\text { 95-percent confidence } \\
\text { limit }\end{array}$} & \multirow{2}{*}{ Estimate } & \multicolumn{2}{|c|}{$\begin{array}{l}\text { 95-percent confidence } \\
\text { limit }\end{array}$} \\
\hline & & Low & High & & Low & High & & Low & High & & Low & High \\
\hline \multicolumn{13}{|c|}{ Souris River Basin-Continued } \\
\hline $1 / 74$ & .2 & .2 & 2.4 & $10,400^{\mathrm{s}}$ & 7,410 & 16,100 & $14,900^{\mathrm{s}}$ & 10,200 & 24,100 & $31,200^{s}$ & 19,800 & 56,200 \\
\hline $4 / 55$ & 8.6 & 3.9 & 10 & $6,330^{s}$ & 3,780 & 12,300 & $8,460^{s}$ & 4,900 & 17,200 & $14,500^{s}$ & 7,910 & 32,200 \\
\hline $2 / 48$ & 7.0 & 1.5 & 6.6 & $13,900^{\mathrm{s}}$ & 5,170 & 50,000 & $21,500^{\mathrm{s}}$ & 7,650 & 83,500 & $46,800^{\mathrm{s}}$ & 15,300 & 208,000 \\
\hline $1 / 75$ & $<0.2$ & .2 & 2.4 & $14,500^{\mathrm{s}}$ & 9,270 & 25,000 & $18,700^{\mathrm{s}}$ & 11,700 & 33,500 & $29,800^{\mathrm{s}}$ & 17,900 & 56,700 \\
\hline \multicolumn{13}{|c|}{ Upper Mississippi River Basin (upstream of the mouth of the Ohio River), excluding the Missouri River Basin } \\
\hline $4 / 27$ & 9.6 & 8.0 & 20 & 5,520 & 3,650 & 8,360 & 6,510 & 4,090 & 10,400 & 9,050 & 4,990 & 16,400 \\
\hline $9 / 90$ & 7.6 & 6.9 & 13 & 16,300 & 12,500 & 21,200 & 18,800 & 13,800 & 25,500 & 24,400 & 16,300 & 36,700 \\
\hline $3 / 33$ & 8.5 & 4.3 & 13 & 1,110 & 786 & 1,560 & 1,260 & 846 & 1,870 & 1,610 & 964 & 2,700 \\
\hline $1 / 15$ & 4.6 & 1.2 & 11 & 326 & 268 & 397 & 347 & 277 & 435 & 394 & 293 & 531 \\
\hline $5 / 74$ & 7.0 & 3.9 & 9.4 & $5,860^{\mathrm{s}}$ & 4,210 & 10,100 & $7,160^{\mathrm{s}}$ & 4,800 & 13,600 & $10,500^{\mathrm{s}}$ & 5,830 & 25,600 \\
\hline $1 / 26$ & 3.4 & .7 & 6.7 & 1,070 & 700 & 1,630 & 1,330 & 830 & 2,140 & 2,070 & 1,130 & 3,790 \\
\hline $7 / 79$ & 6.8 & 5.7 & 12 & 4,990 & 3,510 & 7,090 & 6,290 & 4,230 & 9,360 & 9,950 & 5,960 & 16,600 \\
\hline $4 / 65$ & 4.9 & 3.3 & 8.8 & $29,000^{\mathrm{s}}$ & 19,200 & 71,500 & $38,600^{\mathrm{s}}$ & 23,800 & 122,000 & $69,500^{\mathrm{s}}$ & 36,100 & 400,000 \\
\hline $6 / 102$ & 4.0 & 3.6 & 8.0 & $32,800^{\mathrm{s}}$ & 22,600 & 62,500 & $43,900^{\mathrm{s}}$ & 28,500 & 97,100 & $79,400^{\mathrm{s}}$ & 43,600 & 251,000 \\
\hline $8 / 80$ & 7.6 & 6.7 & 13 & 10,500 & 7,370 & 15,000 & 12,800 & 8,550 & 19,300 & 18,700 & 11,000 & 31,800 \\
\hline $6 / 86$ & 6.8 & 4.3 & 9.4 & 11,900 & 8,100 & 17,400 & 14,900 & 9,690 & 23,000 & 23,000 & 13,100 & 40,300 \\
\hline $4 / 40$ & 9.3 & 5.4 & 14 & 17,100 & 11,100 & 26,300 & 21,300 & 13,200 & 34,600 & 33,100 & 17,900 & 60,900 \\
\hline $6 / 85$ & 6.1 & 4.4 & 9.6 & 22,200 & 16,000 & 30,800 & 27,500 & 19,000 & 39,900 & 41,700 & 25,700 & 67,600 \\
\hline $6 / 44$ & 7.3 & 8.5 & 18 & 14,700 & 10,100 & 21,500 & 18,000 & 11,800 & 27,400 & 26,500 & 15,500 & 45,200 \\
\hline $7 / 98$ & 8.1 & 4.6 & 9.5 & $26,500^{\mathrm{s}}$ & 20,800 & 36,200 & $30,400^{\mathrm{s}}$ & 22,400 & 44,100 & $39,200^{\text {s }}$ & 24,500 & 66,200 \\
\hline $2 / 15$ & 5.1 & 4.9 & 20 & 4,720 & 2,350 & 9,510 & 5,960 & 2,720 & 13,100 & 9,380 & 3,510 & 25,100 \\
\hline $4 / 40$ & 9.8 & 5.4 & 14 & 7,160 & 5,160 & 9,920 & 8,360 & 5,780 & 12,100 & 11,500 & 7,120 & 18,500 \\
\hline $4 / 69$ & 7.0 & 3.1 & 8.3 & 20,000 & 14,900 & 26,800 & 23,600 & 16,800 & 33,000 & 32,500 & 20,900 & 50,500 \\
\hline
\end{tabular}




\section{$38 \quad$ Annual Exceedance Probabilities and Trends for the Central United States during the 2011 Floods}

Table 1. Peak streamflow data and results of flood-probability analyses for selected streamgages in the Central United States affected by 2011 flooding.-Continued

$\left[\mathrm{mi}^{2}\right.$, square miles; $\mathrm{ft}^{3} / \mathrm{s}$, cubic feet per second; AEP, annual exceedance probability; n/a, not applicable; --, data not available; <, less than; >, greater than] [Stations used in the trend analysis are italicized; Stations used in the trend analysis that overlap with trend analyses of Holmes and others (2010) are italicized and bold]

\section{Flood data}

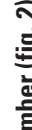

Contri-
buting
drainage
area
$\left(\mathrm{mi}^{2}\right)$

Previous maximum streamflow

Floods of 2011

Site

Station name

buting

(mi)

Date
(month/
year) $\quad$ Stage $(\mathrm{ft}) \quad \begin{gathered}\text { Streamflow } \\ \left(\mathrm{ft}^{3} / \mathrm{s}\right)\end{gathered}$

\begin{tabular}{|c|c|c|c|}
\hline $\begin{array}{c}\text { Rank }^{c} / \\
\text { annual } \\
\text { peaks } \\
\text { in }\end{array}$ & $\begin{array}{l}\text { Date of } \\
\text { peak } \\
\text { stream- } \\
\text { flow }\end{array}$ & $\begin{array}{c}\text { Peak stage } \\
\text { (ft) }\end{array}$ & $\begin{array}{c}\text { Peak } \\
\text { streamflow } \\
\left(\mathrm{ft}^{3} / \mathrm{s}\right)\end{array}$ \\
\hline
\end{tabular}

\begin{tabular}{|c|c|c|c|c|c|c|c|c|c|c|}
\hline \multicolumn{11}{|c|}{ Upper Mississippi River Basin (upstream of the mouth of the Ohio River), excluding the Missouri River Basin-Continued } \\
\hline 82 & 05325000 & $\begin{array}{l}\text { Minnesota River at Mankato, } \\
\text { Minnesota }\end{array}$ & 14,900 & $04 / 1965$ & 29.09 & 94,100 & $8 / 109$ & $3 / 26 / 2011$ & 25.51 & 64,900 \\
\hline 83 & 05330000 & $\begin{array}{l}\text { Minnesota River near Jordan, } \\
\text { Minnesota }\end{array}$ & 16,200 & $04 / 1965$ & $33.89^{f g}$ & 117,000 & $7 / 77$ & $3 / 28 / 2011$ & 31.81 & 72,300 \\
\hline 84 & 05331000 & $\begin{array}{l}\text { Mississippi River at Saint Paul, } \\
\text { Minnesota }\end{array}$ & 36,800 & $04 / 1965$ & 26.01 & $171,000^{d}$ & $7 / 119$ & $3 / 29 / 2011$ & $19.04^{e}$ & $103,000^{\circ}$ \\
\hline 86 & 05344500 & $\begin{array}{l}\text { Mississippi River at Prescott, } \\
\text { Wisconsin }\end{array}$ & 44,800 & $04 / 1965$ & 43.11 & 228,000 & $8 / 83$ & $4 / 12 / 2011$ & $36.76^{e}$ & 125,000 \\
\hline 87 & 05348550 & $\begin{array}{l}\text { Cannon River below Sabre Lake } \\
\text { near Kilkenny, Minnesota }\end{array}$ & 87.9 & $04 / 2001$ & 13.83 & 563 & $3 / 26$ & $7 / 16 / 2011$ & 13.91 & 497 \\
\hline 90 & 05378500 & $\begin{array}{l}\text { Mississippi River at Winona, } \\
\text { Minnesota }\end{array}$ & 59,200 & $04 / 1965$ & 20.77 & 268,000 & $7 / 83$ & $4 / 15 / 2011$ & 16.58 & 170,000 \\
\hline 92 & 05389500 & $\begin{array}{l}\text { Mississippi River at McGregor, } \\
\text { Iowa }\end{array}$ & 67,500 & $04 / 1965$ & 25.38 & $276,000^{\mathrm{d}, 1}$ & $10 / 74$ & $4 / 17 / 2011$ & $21.38^{\mathrm{e}}$ & $182,000^{\circ}$ \\
\hline 93 & 05394500 & $\begin{array}{l}\text { Prairie River Near Merrill, } \\
\quad \text { Wisconsin }\end{array}$ & 184 & 08/1941 & 9.45 & 5,800 & $7 / 90$ & $4 / 12 / 2011$ & 7.62 & 2,550 \\
\hline 94 & 05398000 & $\begin{array}{l}\text { Wisconsin River at Rothschild, } \\
\text { Wisconsin }\end{array}$ & 4,020 & 09/1941 & 22.30 & $75,000^{\mathrm{d}, \mathrm{m}}$ & $6 / 68$ & $4 / 11 / 2011$ & 28.17 & $48,000^{\circ}$ \\
\hline 97 & 05414350 & $\begin{array}{l}\text { Little Maquoketa River near } \\
\quad \text { Graf, Iowa }\end{array}$ & 39.6 & $06 / 2008$ & 16.47 & 8,370 & $1 / 61$ & $7 / 28 / 2011$ & 17.48 & 9,420 \\
\hline 98 & 05414400 & $\begin{array}{l}\text { Middle Fork Little Maquoketa } \\
\text { River near Rickardsville, Iowa }\end{array}$ & 30.2 & 08/1972 & 27.70 & 23,000 & $8 / 58$ & $7 / 28 / 2011$ & 19.81 & 7,510 \\
\hline 99 & 05414450 & $\begin{array}{l}\text { North Fork Little Maquoketa } \\
\text { River near Rickardsville, Iowa }\end{array}$ & 21.6 & 06/2008 & 12.58 & 8,040 & $3 / 61$ & $7 / 28 / 2011$ & 14.88 & 6,690 \\
\hline 100 & 05414605 & $\begin{array}{l}\text { Bloody Run Tributary near Sher- } \\
\text { rill, Iowa }\end{array}$ & .6 & $06 / 2008$ & 22.71 & 1,110 & $3 / 21$ & $7 / 28 / 2011$ & 18.63 & 624 \\
\hline 101 & 05418400 & $\begin{array}{l}\text { North Fork Maquoketa River } \\
\text { near Fulton, Iowa }\end{array}$ & 505 & $07 / 2010$ & 22.44 & 25,000 & $4 / 13$ & $7 / 29 / 2011$ & 19.53 & 17,100 \\
\hline 102 & 05420500 & $\begin{array}{l}\text { Mississippi River at Clinton, } \\
\text { Iowa }\end{array}$ & 85,600 & 04/1965 & 24.65 & $307,000^{\mathrm{d}, 1}$ & $10 / 138$ & $4 / 20 / 2011$ & $21.93^{\mathrm{e}}$ & 229,000 \\
\hline 107 & 05451080 & $\begin{array}{l}\text { South Fork Iowa River near } \\
\text { Blairsburg, Iowa }\end{array}$ & 12.0 & $06 / 2008$ & 12.50 & 762 & $3 / 6$ & $2 / 16 / 2011$ & 11.14 & 564 \\
\hline 111 & 05465150 & $\begin{array}{l}\text { North Fork Long Creek at Ain- } \\
\text { sworth, Iowa }\end{array}$ & 30.2 & 06/1990 & 90.66 & $5,800^{\mathrm{j}}$ & $7 / 48$ & $6 / 15 / 2011$ & 91.88 & 3,330 \\
\hline 113 & 05469350 & Haight Creek at Kingston, Iowa & 2.7 & $05 / 2010$ & 20.17 & 3,170 & $2 / 22$ & $6 / 15 / 2011$ & 19.60 & 2,940 \\
\hline 114 & 05474500 & $\begin{array}{l}\text { Mississippi River at Keokuk, } \\
\text { Iowa }\end{array}$ & 119,000 & 07/1993 & 27.58 & $446,000^{\mathrm{d}, \mathrm{t}}$ & $11 / 134$ & $4 / 25 / 2011$ & 18.98 & $276,000^{\mathrm{d}, \mathrm{l}, \mathrm{t}}$ \\
\hline 115 & 05474900 & $\begin{array}{l}\text { Elk Creek On CSAH } 1 \text { near } \\
\text { Brewster, Minnesota }\end{array}$ & 61.0 & $05 / 2001$ & 25.59 & 4,360 & $2 / 11$ & $7 / 12 / 2011$ & 25.29 & 3,680 \\
\hline 116 & 05476000 & $\begin{array}{l}\text { Des Moines River at Jackson, } \\
\text { Minnesota }\end{array}$ & 1,250 & $04 / 1969$ & 19.45 & 15,700 & $7 / 86$ & $3 / 26 / 2011$ & 15.69 & 6,860 \\
\hline 121 & 05486490 & $\begin{array}{l}\text { Middle River near Indianola, } \\
\quad \text { Iowa }\end{array}$ & 489 & $06 / 1947$ & $26.40^{f}$ & 34,000 & $4 / 72$ & $6 / 10 / 2011$ & 24.08 & 15,100 \\
\hline 122 & 05488110 & $\begin{array}{l}\text { Des Moines River near Pella, } \\
\text { Iowa }\end{array}$ & 12,330 & 07/1993 & 109.71 & $105,000^{\mathrm{d}}$ & $5 / 19$ & $7 / 20 / 2011$ & 96.39 & $31,000^{\circ}$ \\
\hline
\end{tabular}




\begin{tabular}{|c|c|c|c|c|c|c|c|c|c|c|c|c|}
\hline \multirow{4}{*}{$\begin{array}{l}\text { Rank/ } \\
\text { annual } \\
\text { peaks } \\
\text { used in } \\
\text { analy- } \\
\text { sis }^{\text {ff }}\end{array}$} & \multirow{2}{*}{\multicolumn{3}{|c|}{$\begin{array}{l}\text { AEP for observed } 2011 \text { flood } \\
\text { (percent) })^{b}\end{array}$}} & \multicolumn{9}{|c|}{ Expected peak streamflows for selected AEP $\left(\mathrm{ft}^{3} / \mathrm{s}\right)^{\mathrm{a}}$} \\
\hline & & & & \multicolumn{3}{|c|}{$\begin{array}{c}\text { 2-percent AEP } \\
\text { (50-year recurrence) }\end{array}$} & \multicolumn{3}{|c|}{$\begin{array}{c}\text { 1-percent AEP } \\
\text { (100-year recurrence) }\end{array}$} & \multicolumn{3}{|c|}{$\begin{array}{c}\text { 0.2-percent AEP } \\
\text { (500-year recurrence) }\end{array}$} \\
\hline & \multirow{2}{*}{$\begin{array}{l}\text { Esti- } \\
\text { mate }\end{array}$} & \multicolumn{2}{|c|}{$\begin{array}{l}\text { Typical range for } \\
66.7-\text { percent } \\
\text { confidence level }\end{array}$} & \multirow{2}{*}{ Estimate } & \multicolumn{2}{|c|}{$\begin{array}{l}\text { 95-percent confidence } \\
\text { limit }\end{array}$} & \multirow{2}{*}{ Estimate } & \multicolumn{2}{|c|}{$\begin{array}{l}\text { 95-percent confidence } \\
\text { limit }\end{array}$} & \multirow{2}{*}{ Estimate } & \multicolumn{2}{|c|}{$\begin{array}{l}\text { 95-percent confidence } \\
\text { limit }\end{array}$} \\
\hline & & Low & High & & Low & High & & Low & High & & Low & High \\
\hline \multicolumn{13}{|c|}{ Upper Mississippi River Basin (upstream of the mouth of the Ohio River), excluding the Missouri River Basin-Continued } \\
\hline $8 / 109$ & 3.3 & 4.9 & 9.6 & $72,400^{s}$ & 57,600 & 97,700 & $83,000^{s}$ & 62,600 & 118,000 & $106,000^{s}$ & 70,400 & 175,000 \\
\hline 7/77 & 4.9 & 5.9 & 12 & $95,400^{s}$ & 76,000 & 127,000 & $117,000^{s}$ & 91,100 & 159,000 & $175,000^{s}$ & 131,000 & 252,000 \\
\hline $7 / 119$ & 5.0 & 3.8 & 7.8 & $123,000^{s}$ & 107,000 & 146,000 & $140,000^{s}$ & 120,000 & 167,000 & $177,000^{s}$ & 150,000 & 217,000 \\
\hline $8 / 83$ & 9.1 & 6.5 & 13 & $172,000^{5}$ & 149,000 & 206,000 & $194,000^{5}$ & 166,000 & 235,000 & $246,000^{s}$ & 206,000 & 307,000 \\
\hline $3 / 26$ & 9.1 & 5.5 & 17 & 676 & 504 & 906 & 764 & 547 & 1,070 & 976 & 632 & 1,510 \\
\hline $7 / 83$ & 8.7 & 5.5 & 11 & $224,000^{s}$ & 197,000 & 262,000 & $250,000^{s}$ & 218,000 & 296,000 & $310,000^{s}$ & 264,000 & 377,000 \\
\hline$--/--$ & -- & -- & -- & $226,000^{q}$ & -- & -- & $251,000^{q}$ & -- & -- & $309,000^{q}$ & -- & -- \\
\hline $7 / 89$ & 7.2 & 5.1 & 10 & 3,210 & 2,730 & 3,770 & 3,610 & 2,990 & 4,370 & $5,310^{s}$ & 4,500 & 6,530 \\
\hline $5 / 67$ & 8.5 & 4.4 & 10 & $59,300^{\text {s }}$ & 52,000 & 69,900 & $64,200^{\mathrm{s}}$ & 55,900 & 76,500 & $74,200^{\text {s }}$ & 63,600 & 90,300 \\
\hline $1 / 61$ & 1.9 & .3 & 2.9 & 9,290 & 6,950 & 12,400 & 11,300 & 8,130 & 15,600 & 16,600 & 10,900 & 25,400 \\
\hline $8 / 58$ & 7.3 & 9.3 & 18 & 11,700 & 8,860 & 15,600 & 14,400 & 10,500 & 19,800 & 21,800 & 14,400 & 33,100 \\
\hline $3 / 61$ & 2.0 & 2.3 & 7.3 & 6,710 & 4,810 & 9,370 & 8,210 & 5,670 & 11,900 & 12,300 & 7,720 & 19,700 \\
\hline $3 / 21$ & 9.9 & 6.8 & 20 & $1,540^{\mathrm{s}}$ & 861 & 3,830 & $2,150^{\mathrm{s}}$ & 1,140 & 5,940 & $4,300^{\mathrm{s}}$ & 2,010 & 14,900 \\
\hline $4 / 13$ & 8.1 & 17 & 40 & 24,900 & 19,000 & 32,600 & 28,900 & 21,400 & 39,200 & 39,000 & 26,400 & 57,600 \\
\hline$--/--$ & -- & -- & -- & $259,000^{q}$ & -- & -- & $283,000^{q}$ & -- & -- & $337,000^{q}$ & -- & -- \\
\hline$--/--$ & -- & -- & -- & $644^{r}$ & -- & -- & $743^{r}$ & -- & -- & $982^{r}$ & -- & -- \\
\hline $7 / 48$ & 9.6 & 9.5 & 19 & 5,700 & 4,120 & 7,870 & 6,840 & 4,790 & 9,780 & 9,840 & 6,270 & 15,500 \\
\hline $2 / 22$ & 2.8 & 3.3 & 14 & 3,230 & 2,220 & 4,720 & 3,950 & 2,620 & 5,970 & 5,980 & 3,590 & 9,960 \\
\hline$--/--$ & -- & -- & -- & $331,000^{q}$ & -- & -- & $366,000^{q}$ & -- & -- & $428,000^{q}$ & -- & -- \\
\hline $2 / 11$ & 2.3 & 6.7 & 27 & 3,820 & 1,940 & 7,520 & 4,700 & 2,270 & 9,770 & 7,170 & 2,980 & 17,200 \\
\hline $7 / 86$ & 7.3 & 5.3 & 11 & 10,400 & 7,660 & 14,000 & 12,500 & 8,880 & 17,700 & 18,100 & 11,500 & 28,500 \\
\hline $4 / 72$ & 9.8 & 3.0 & 7.9 & 21,200 & 17,400 & 25,800 & 23,800 & 18,900 & 29,900 & 29,600 & 21,800 & 40,200 \\
\hline$--/--$ & -- & -- & -- & $68,700^{\text {u }}$ & -- & -- & $93,600^{\text {и }}$ & -- & -- & $137,000^{\text {u }}$ & -- & -- \\
\hline
\end{tabular}




\section{Annual Exceedance Probabilities and Trends for the Central United States during the 2011 Floods}

Table 1. Peak streamflow data and results of flood-probability analyses for selected streamgages in the Central United States affected by 2011 flooding.-Continued

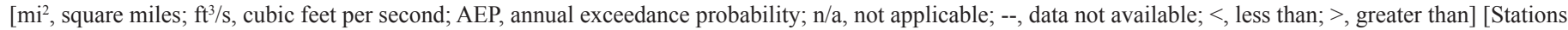
used in the trend analysis are italicized; Stations used in the trend analysis that overlap with trend analyses of Holmes and others (2010) are italicized and bold]

\section{Flood data}

Contri-
buting
drainage
area
$\left(\mathrm{mi}^{2}\right)$

Station name

\section{Previous maximum streamflow}

Site number

area
$\left(\mathrm{mi}^{2}\right)$

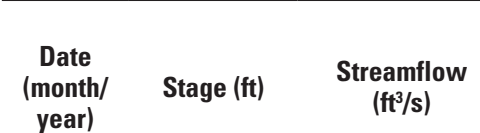

Floods of 2011

\begin{tabular}{|c|c|c|c|}
\hline $\begin{array}{l}\text { Rankc / }^{c} \\
\text { annual } \\
\text { peaks } \\
\text { in } \\
\text { record }\end{array}$ & $\begin{array}{l}\text { Date of } \\
\text { peak } \\
\text { stream- } \\
\text { flow }\end{array}$ & $\begin{array}{c}\text { Peak stage } \\
\text { (ft) }\end{array}$ & $\begin{array}{c}\text { Peak } \\
\text { streamflow } \\
\left(\mathrm{ft}^{3} / \mathrm{s}\right)\end{array}$ \\
\hline
\end{tabular}

\begin{tabular}{|c|c|c|c|c|c|c|c|c|c|c|}
\hline \multicolumn{11}{|c|}{ Upper Mississippi River Basin (upstream of the mouth of the Ohio River), excluding the Missouri River Basin-Continued } \\
\hline 123 & 05488500 & $\begin{array}{l}\text { Des Moines River near Tracy, } \\
\text { Iowa }\end{array}$ & 12,479 & $06 / 1947$ & 26.50 & 155,000 & $34 / 93$ & $6 / 28 / 2011$ & 13.27 & $33,500^{\mathrm{d}}$ \\
\hline 124 & 05489500 & $\begin{array}{l}\text { Des Moines River at Ottumwa, } \\
\text { Iowa }\end{array}$ & 13,374 & $05 / 1903$ & 19.40 & $140,000^{\mathrm{j}}$ & $16 / 96$ & $6 / 15 / 2011$ & 14.90 & $49,400^{\mathrm{d}}$ \\
\hline 125 & 05490500 & $\begin{array}{l}\text { Des Moines River at Keosauqua, } \\
\text { Iowa }\end{array}$ & 14,038 & $06 / 1903$ & 27.85 & 146,000 & $13 / 104$ & $6 / 15 / 2011$ & $25.81^{\mathrm{e}}$ & $69,900^{\mathrm{d}}$ \\
\hline 126 & 05495000 & Fox River at Wayland, Missouri & 400 & $04 / 1973$ & 21.71 & 26,400 & $2 / 90$ & $6 / 15 / 2011$ & 23.07 & 26,200 \\
\hline 127 & 05496000 & $\begin{array}{l}\text { Wyaconda River above Canton, } \\
\text { Missouri }\end{array}$ & 393 & 06/1933 & $30.00^{j}$ & 17,700 & $6 / 86$ & $6 / 16 / 2011$ & 28.25 & 13,700 \\
\hline 139 & 05587450 & $\begin{array}{l}\text { Mississippi River at Grafton, } \\
\text { Illinois }\end{array}$ & 171,300 & $08 / 1993$ & 38.17 & $598,000^{\mathrm{d}}$ & $7 / 25$ & $5 / 1 / 2011$ & 25.93 & $362,000^{\mathrm{d}}$ \\
\hline 140 & 05592100 & $\begin{array}{l}\text { Kaskaskia River at Cowden, } \\
\text { Illinois }\end{array}$ & 1,330 & $05 / 2002$ & 20.36 & $25,300^{\mathrm{d}}$ & $3 / 41$ & $4 / 26 / 2011$ & 18.88 & $17,000^{\mathrm{d}}$ \\
\hline 142 & 05592575 & $\begin{array}{l}\text { Hickory Creek near Brown- } \\
\text { stown, Illinois }\end{array}$ & 44.2 & $11 / 1993$ & 16.43 & 6,250 & $1 / 23$ & $4 / 26 / 2011$ & 16.88 & 6,880 \\
\hline 145 & 05599490 & $\begin{array}{l}\text { Big Muddy River at Route } 127 \text { at } \\
\text { Murphysboro, Illinois }\end{array}$ & 2,159 & $03 / 2008$ & 37.24 & $31,500^{\mathrm{d}}$ & $5 / 84$ & $5 / 2 / 2011$ & $40.47^{e}$ & $42,400^{d}$ \\
\hline 146 & 07010000 & $\begin{array}{l}\text { Mississippi River at St. Louis, } \\
\text { Missouri }\end{array}$ & 697,000 & 08/1993 & 49.58 & $1,080,000^{\mathrm{d}}$ & $45 / 151$ & $4 / 30 / 2011$ & 34.93 & $615,000^{\mathrm{d}}$ \\
\hline 148 & 07020500 & $\begin{array}{l}\text { Mississippi River at Chester, } \\
\text { Illinois }\end{array}$ & 708,600 & $04 / 1927$ & 34.40 & $1,060,000^{\mathrm{d}, 1}$ & $18 / 87$ & $5 / 2 / 2011$ & 39.74 & $703,000^{d}$ \\
\hline 149 & 07022000 & $\begin{array}{l}\text { Mississippi River at Thebes, } \\
\text { Illinois }\end{array}$ & 713,200 & $07 / 1844$ & -- & $1,075,000^{\mathrm{d}, \mathrm{m}}$ & $5 / 80$ & $5 / 2 / 2011$ & $45.52^{\mathrm{e}}$ & $876,000^{\mathrm{d}}$ \\
\hline \multicolumn{11}{|c|}{ Missouri River Basin } \\
\hline 150 & 06012500 & $\begin{array}{l}\text { Red Rock River below Lima } \\
\text { Reservoir near Monida, } \\
\text { Montana }\end{array}$ & 570 & $05 / 1933$ & 5.40 & $2,500^{\mathrm{d}}$ & $10 / 87$ & $6 / 13 / 2011$ & 4.03 & $860^{\mathrm{d}}$ \\
\hline 151 & 06015430 & $\begin{array}{l}\text { Clark Canyon near Dillon, } \\
\text { Montana }\end{array}$ & 18.0 & $05 / 1984$ & 7.10 & 415 & $2 / 39$ & $6 / 8 / 2011$ & 6.10 & 315 \\
\hline 152 & 06019500 & $\begin{array}{l}\text { Ruby River above reservoir near } \\
\text { Alder, Montana }\end{array}$ & 534 & $05 / 1984$ & 6.24 & 3,810 & $5 / 73$ & $6 / 8 / 2011$ & 6.07 & 1,780 \\
\hline 153 & 06020600 & $\begin{array}{l}\text { Ruby River below reservoir near } \\
\text { Alder, Montana }\end{array}$ & 596 & $05 / 1984$ & 8.52 & $3,010^{\mathrm{d}}$ & $3 / 49$ & $6 / 9 / 2011$ & 6.31 & $1,720^{\mathrm{d}}$ \\
\hline 154 & 06023000 & $\begin{array}{l}\text { Ruby River near Twin Bridges, } \\
\text { Montana }\end{array}$ & 965 & $06 / 1947$ & 6.89 & 1,500 & $1 / 27$ & $7 / 1 / 2011$ & 5.81 & 1,630 \\
\hline 155 & 06023100 & $\begin{array}{l}\text { Beaverhead River at Twin } \\
\text { Bridges, Montana }\end{array}$ & 4,779 & $06 / 2010$ & 5.52 & 2,830 & $2 / 3$ & $7 / 1 / 2011$ & 5.50 & 2,800 \\
\hline 156 & 06024540 & $\begin{array}{l}\text { Big Hole River below Mudd } \\
\text { Creek near Wisdom, Montana }\end{array}$ & 1,267 & $06 / 2010$ & 6.47 & 5,580 & $1 / 14$ & $6 / 10 / 2011$ & 6.68 & 6,660 \\
\hline 157 & 06024580 & $\begin{array}{l}\text { Big Hole River near Wise River, } \\
\text { Montana }\end{array}$ & 1,611 & $06 / 2010$ & 6.85 & 8,100 & $1 / 5$ & $6 / 11 / 2011$ & 7.14 & 9,130 \\
\hline 158 & 06026210 & $\begin{array}{l}\text { Big Hole River near Glen, } \\
\text { Montana }\end{array}$ & 2,655 & $06 / 2010$ & 7.16 & 10,400 & $1 / 14$ & $6 / 10 / 2011$ & 7.63 & 11,900 \\
\hline 159 & 06031950 & $\begin{array}{l}\text { Cataract Creek near Basin, } \\
\text { Montana }\end{array}$ & 30.6 & 05/1981 & 6.88 & 3,150 & $3 / 39$ & $6 / 7 / 2011$ & 3.38 & 820 \\
\hline
\end{tabular}




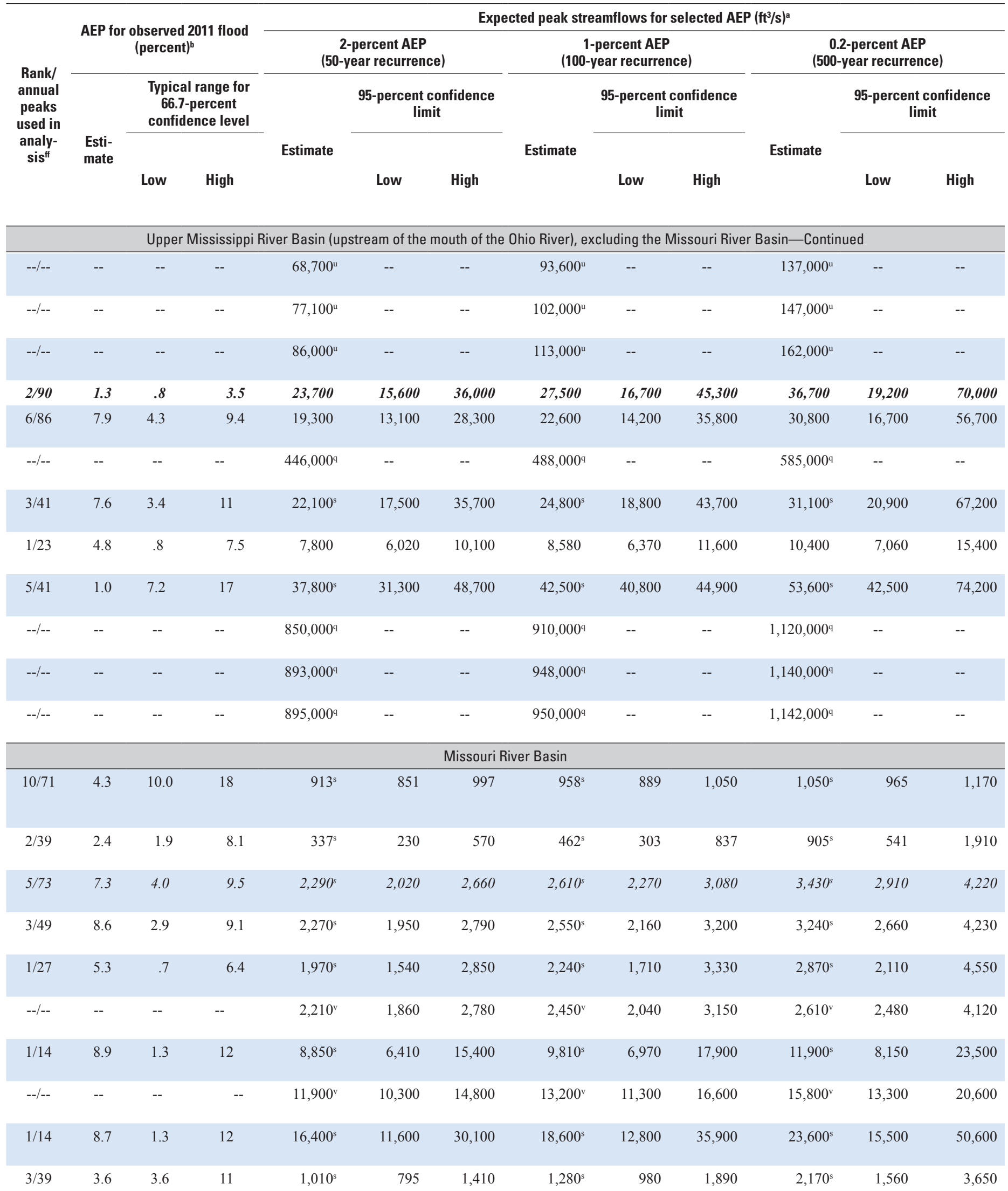




\section{Annual Exceedance Probabilities and Trends for the Central United States during the 2011 Floods}

Table 1. Peak streamflow data and results of flood-probability analyses for selected streamgages in the Central United States affected by 2011 flooding.-Continued

$\left[\mathrm{mi}^{2}\right.$, square miles; $\mathrm{ft}^{3} / \mathrm{s}$, cubic feet per second; AEP, annual exceedance probability; n/a, not applicable; --, data not available; <, less than; >, greater than] [Stations used in the trend analysis are italicized; Stations used in the trend analysis that overlap with trend analyses of Holmes and others (2010) are italicized and bold]

Flood data

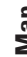

Contri-

buting

Station name drainage

area
Previous maximum streamflow

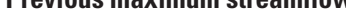

$\left(\mathrm{mi}^{2}\right)$

\section{Date (month/ year)}

Missouri River Basin-Continued

16006033000 Boulder River near Boulder Montana

$16106036650 \quad$ Jefferson River near Three Forks, Montana

16206036905 Firehole River near West Yellowstone, Montana

16306037500 Madison River near West Yellowstone, Montana

$16406040800 \quad$ Madison River above powerplan near McAllister, Montana

$16606043500 \quad$ Gallatin River near Gallatin Gateway, Montana

\begin{tabular}{|c|c|c|c|c|c|c|c|c|c|c|}
\hline 167 & 06052500 & $\begin{array}{l}\text { Gallatin River at Logan, Mon- } \\
\quad \text { tana }\end{array}$ & 1,795 & $06 / 1899$ & -- & 9,840 & $7 / 92$ & $6 / 30 / 2011$ & $9.57^{h, e}$ & 7,980 \\
\hline 168 & 06054500 & $\begin{array}{l}\text { Missouri River at Toston, } \\
\text { Montana }\end{array}$ & 14,669 & 06/1997 & 12.22 & 34,000 & $4 / 79$ & $6 / 15 / 2011$ & 11.53 & 29,500 \\
\hline 169 & 06056300 & $\begin{array}{l}\text { Cabin Creek near Townsend, } \\
\quad \text { Montana }\end{array}$ & 11.8 & $06 / 1960$ & 2.00 & 70 & $1 / 52$ & $5 / 9 / 2011$ & 3.49 & 120 \\
\hline 170 & 06061500 & $\begin{array}{l}\text { Prickly Pear Creek near Clancy, } \\
\text { Montana }\end{array}$ & 192 & 05/1981 & 8.82 & 2,300 & $3 / 71$ & 6/7/2011 & 5.37 & 1,030 \\
\hline 171 & 06062500 & $\begin{array}{l}\text { Tenmile Creek near Rimini, } \\
\quad \text { Montana }\end{array}$ & 30.9 & $05 / 1981$ & 6.20 & 3,290 & $4 / 95$ & $6 / 7 / 2011$ & 4.31 & 742 \\
\hline 172 & 06071300 & $\begin{array}{l}\text { Little Prickly Pear Creek at Wolf } \\
\text { Creek, Montana }\end{array}$ & 381 & $05 / 1975$ & 7.45 & $4,500^{\mathrm{m}}$ & $3 / 26$ & 6/9/2011 & 7.84 & 2,460 \\
\hline 173 & 06076690 & $\begin{array}{l}\text { Smith River near Fort Logan, } \\
\text { Montana }\end{array}$ & 846 & 05/1981 & 7.80 & 4,600 & $3 / 23$ & $6 / 10 / 2011$ & 6.70 & 2,270 \\
\hline 174 & 06077200 & $\begin{array}{l}\text { Smith River below Eagle Creek } \\
\text { near Fort Logan, Montana }\end{array}$ & 1,088 & 06/1997 & $7.00^{\mathrm{g}}$ & 3,900 & $1 / 15$ & 6/9/2011 & 7.65 & 4,030 \\
\hline 175 & 06077500 & Smith River near Eden, Montana & 1,594 & $06 / 1953$ & 10.46 & 12,300 & $3 / 27$ & $6 / 10 / 2011$ & 8.77 & 5,710 \\
\hline 176 & 06078200 & $\begin{array}{l}\text { Missouri River near Ulm, } \\
\text { Montana }\end{array}$ & 20,941 & $06 / 1953$ & 17.00 & $35,000^{\mathrm{d}, \mathrm{w}, \mathrm{m}}$ & $3 / 56$ & $6 / 11 / 2011$ & 15.48 & $28,800^{\mathrm{d}}$ \\
\hline 177 & 06089000 & Sun River near Vaughn, Montana & 1,849 & 06/1964 & 23.40 & 53,500 & $4 / 78$ & $6 / 10 / 2011$ & 8.29 & 14,800 \\
\hline 178 & 06090300 & $\begin{array}{l}\text { Missouri River near Great Falls, } \\
\text { Montana }\end{array}$ & 23,292 & $06 / 1964$ & -- & $72,000^{\mathrm{d}, 1}$ & $5 / 60$ & $6 / 11 / 2011$ & 8.10 & $38,800^{\mathrm{d}}$ \\
\hline 179 & 06090650 & Lake Creek near Power, Montana & 83.8 & 03/1993 & 7.30 & $300^{1}$ & $2 / 22$ & $6 / 7 / 2011$ & 3.53 & 223 \\
\hline 180 & 06090800 & $\begin{array}{l}\text { Missouri River at Fort Benton, } \\
\quad \text { Montana }\end{array}$ & 24,749 & 06/1908 & $18.50^{\circ}$ & $140,000^{j}$ & $10 / 120$ & $6 / 10 / 2011$ & $11.79^{h, e}$ & $52,700^{d}$ \\
\hline 181 & 06091700 & $\begin{array}{l}\text { Two Medicine River below } \\
\text { South Fork, Near Browning, } \\
\text { Montana }\end{array}$ & 250 & 05/1991 & 7.78 & 11,700 & $4 / 35$ & $6 / 7 / 2011$ & 7.94 & 6,450 \\
\hline 182 & 06099500 & $\begin{array}{l}\text { Marias River near Shelby, } \\
\quad \text { Montana }\end{array}$ & 3,242 & 06/1964 & 23.64 & $241,000^{p}$ & $10 / 105$ & $6 / 10 / 2011$ & 12.11 & 18,800 \\
\hline 184 & 06108800 & Teton River at Loma, Montana & 2,010 & $06 / 2002$ & 5.87 & 2,000 & $1 / 14$ & $6 / 12 / 2011$ & 8.52 & 3,910 \\
\hline
\end{tabular}

$7,000^{\mathrm{m}}$

17,000

$9,532 \quad 06 / 1995$

$282 \quad 05 / 1996$

$420 \quad 05 / 1996$

$2,186 \quad 06 / 2010$

$825 \quad 06 / 1997$

9.53

6.10

3.78

2,050

2,820

$4,220^{\mathrm{d}}$

9,160

6.71

2/72

$6 / 7 / 2011$

$6 / 12 / 2011$

9.38

5.40

3.51

10.80

$6 / 24 / 2011$

6.51

6/30/2011

6.51

8,410

8,410

,980

, 500
Floods of 2011

$\begin{array}{cccc}\begin{array}{c}\text { Rank }^{c} / \\ \text { annual } \\ \text { peaks } \\ \text { in }\end{array} & \begin{array}{c}\text { Date of } \\ \text { peak } \\ \text { stream- }\end{array} & \begin{array}{c}\text { Peak stage } \\ \text { (ft) }\end{array} & \begin{array}{c}\text { Peak } \\ \text { streamflow } \\ \left(\mathrm{ft}^{3} / \mathbf{s}\right)\end{array} \\ \text { record } & \text { flow } & & \end{array}$




\begin{tabular}{|c|c|c|c|c|c|c|c|c|c|c|c|c|}
\hline \multirow{4}{*}{$\begin{array}{c}\text { Rank/ } \\
\text { annual } \\
\text { peaks } \\
\text { used in } \\
\text { analy- } \\
\text { sis }^{f f}\end{array}$} & \multirow{2}{*}{\multicolumn{3}{|c|}{$\begin{array}{l}\text { AEP for observed } 2011 \text { flood } \\
\text { (percent) }^{\mathrm{b}}\end{array}$}} & \multicolumn{9}{|c|}{ Expected peak streamflows for selected AEP $\left(\mathrm{ft}^{3} / \mathrm{s}\right)^{\mathrm{a}}$} \\
\hline & & & & \multicolumn{3}{|c|}{$\begin{array}{c}\text { 2-percent AEP } \\
\text { (50-year recurrence) }\end{array}$} & \multicolumn{3}{|c|}{$\begin{array}{c}\text { 1-percent AEP } \\
\text { (100-year recurrence) }\end{array}$} & \multicolumn{3}{|c|}{$\begin{array}{c}\text { 0.2-percent AEP } \\
\text { (500-year recurrence) }\end{array}$} \\
\hline & \multirow{2}{*}{$\begin{array}{l}\text { Esti- } \\
\text { mate }\end{array}$} & \multicolumn{2}{|c|}{$\begin{array}{c}\text { Typical range for } \\
66.7 \text {-percent } \\
\text { confidence level }\end{array}$} & \multirow{2}{*}{ Estimate } & \multicolumn{2}{|c|}{$\begin{array}{l}\text { 95-percent confidence } \\
\text { limit }\end{array}$} & \multirow{2}{*}{ Estimate } & \multicolumn{2}{|c|}{$\begin{array}{l}\text { 95-percent confidence } \\
\text { limit }\end{array}$} & \multirow{2}{*}{ Estimate } & \multicolumn{2}{|c|}{$\begin{array}{l}\text { 95-percent confidence } \\
\text { limit }\end{array}$} \\
\hline & & Low & High & & Low & High & & Low & High & & Low & High \\
\hline \multicolumn{13}{|c|}{ Missouri River Basin—Continued } \\
\hline $2 / 72$ & 2.7 & 1.0 & 4.4 & $3,770^{\mathrm{s}}$ & 3,140 & 4,760 & $4,540^{\mathrm{s}}$ & 3,710 & 5,890 & $6,700^{\mathrm{s}}$ & 5,260 & 9,210 \\
\hline $1 / 75$ & 6.5 & .2 & 2.4 & $21,200^{\mathrm{s}}$ & 18,400 & 25,300 & $23,400^{s}$ & 20,100 & 28,400 & $28,300^{\text {s }}$ & 23,900 & 35,200 \\
\hline $2 / 22$ & 6.9 & 3.3 & 14 & $1,930^{\mathrm{s}}$ & 1,610 & 2,560 & $2,160^{\mathrm{s}}$ & 1,770 & 2,960 & $2,730^{\mathrm{s}}$ & 2,150 & 4,030 \\
\hline $3 / 85$ & 2.2 & 1.7 & 5.3 & $2,540^{\mathrm{s}}$ & 2,330 & 2,810 & $2,770^{\mathrm{s}}$ & 2,520 & 3,090 & $3,300^{\mathrm{s}}$ & 2,950 & 3,770 \\
\hline $1 / 10$ & 4.1 & 1.8 & 16 & $6,520^{\mathrm{s}}$ & 5,190 & 10,300 & $7,080^{\mathrm{s}}$ & 5,530 & 11,700 & $8,360^{\mathrm{s}}$ & 6,290 & 15,300 \\
\hline $3 / 98$ & 5.9 & 1.4 & 4.6 & $9,490^{\mathrm{s}}$ & 8,760 & 10,400 & $10,200^{s}$ & 9,350 & 11,300 & $11,700^{s}$ & 10,600 & 13,200 \\
\hline $7 / 92$ & 8.6 & 4.9 & 10 & $9,730^{s}$ & 8,860 & 10,900 & $10,500^{s}$ & 9,520 & 11,900 & $12,200^{s}$ & 10,900 & 14,100 \\
\hline $4 / 79$ & 8.1 & 2.7 & 7.2 & $35,400^{s}$ & 31,900 & 40,300 & $38,100^{s}$ & 34,100 & 43,800 & $43,700^{s}$ & 38,700 & 51,300 \\
\hline $1 / 52$ & 1.0 & .4 & 3.4 & $89^{s}$ & 59 & 152 & $120^{s}$ & 77 & 214 & $215^{s}$ & 129 & 425 \\
\hline $3 / 71$ & 2.0 & 2.0 & 6.3 & $1,030^{\mathrm{s}}$ & 842 & 1,330 & $1,270^{\mathrm{s}}$ & 1,020 & 1,680 & $1,960^{\mathrm{s}}$ & 1,500 & 2,770 \\
\hline $4 / 95$ & 3.6 & 2.3 & 6.0 & $884^{s}$ & 732 & 1,110 & $1,090^{\mathrm{s}}$ & 882 & 1,400 & $1,660^{s}$ & 1,300 & 2,260 \\
\hline $3 / 26$ & 8.6 & 5.5 & 17 & $4,640^{\mathrm{s}}$ & 2,930 & 9,170 & $6,010^{\mathrm{s}}$ & 3,650 & 12,700 & $10,200^{s}$ & 5,680 & 25,000 \\
\hline $3 / 23$ & 9.3 & 6.2 & 19 & $4,450^{\mathrm{s}}$ & 2,930 & 8,460 & $5,840^{\mathrm{s}}$ & 3,680 & 12,000 & $10,400^{s}$ & 5,910 & 25,500 \\
\hline $1 / 15$ & 5.5 & 1.2 & 11 & $5,200^{\mathrm{s}}$ & 3,520 & 10,100 & $6,130^{\mathrm{s}}$ & 4,030 & 12,700 & $8,520^{\mathrm{s}}$ & 5,240 & 20,100 \\
\hline $3 / 27$ & 7.0 & 5.3 & 16 & $8,810^{\mathrm{s}}$ & 6,400 & 14,100 & $11,100^{s}$ & 7,800 & 19,000 & $18,300^{\mathrm{s}}$ & 11,900 & 36,200 \\
\hline $3 / 55$ & 8.7 & 2.6 & 8.1 & $37,800^{\mathrm{s}}$ & 32,100 & 46,900 & $41,900^{s}$ & 35,200 & 52,900 & $51,200^{\mathrm{s}}$ & 42,000 & 66,700 \\
\hline $4 / 78$ & 6.7 & 2.7 & 7.3 & $22,800^{s}$ & 18,800 & 29,100 & $29,400^{s}$ & 23,600 & 38,900 & $51,800^{\circ}$ & 39,100 & 74,200 \\
\hline $5 / 59$ & 10 & 5.0 & 12 & $59,300^{s}$ & 49,300 & 75,200 & $68,900^{s}$ & 56,300 & 89,400 & $93,400^{\mathrm{s}}$ & 73,800 & 127,000 \\
\hline $2 / 22$ & 7.0 & 3.3 & 14 & $335^{\mathrm{s}}$ & 235 & 584 & $415^{\mathrm{s}}$ & 282 & 774 & $651^{s}$ & 409 & 1,400 \\
\hline $10 / 120$ & 5.3 & 5.9 & 11 & $65,900^{s}$ & 54,900 & 83,000 & $76,600^{s}$ & 62,800 & 98,800 & $104,000^{s}$ & 82,400 & 141,000 \\
\hline $4 / 35$ & 9.8 & 6.2 & 16 & $12,200^{s}$ & 9,160 & 18,400 & $15,700^{\mathrm{s}}$ & 11,400 & 25,200 & $27,100^{\mathrm{s}}$ & 18,200 & 49,800 \\
\hline $10 / 105$ & 9.9 & 6.7 & 12 & $47,300^{s}$ & 37,300 & 63,100 & $68,400^{5}$ & 52,500 & 95,700 & $155,000^{5}$ & 111,000 & 243,000 \\
\hline $1 / 14$ & 3.4 & 1.3 & 12 & $5,040^{\mathrm{s}}$ & 2,510 & 17,400 & $7,080^{\mathrm{s}}$ & 3,290 & 28,200 & $14,300^{\mathrm{s}}$ & 5,750 & 78,400 \\
\hline
\end{tabular}




\section{Annual Exceedance Probabilities and Trends for the Central United States during the 2011 Floods}

Table 1. Peak streamflow data and results of flood-probability analyses for selected streamgages in the Central United States affected by 2011 flooding.-Continued

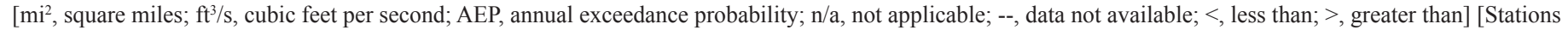
used in the trend analysis are italicized; Stations used in the trend analysis that overlap with trend analyses of Holmes and others (2010) are italicized and bold]

Flood data

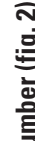

Contri-

buting

Site

number
Station name rainage
Previous maximum streamflow

area
$\left(m i^{2}\right)$

$\left(\mathrm{mi}^{2}\right)$

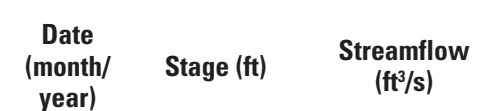

Floods of 2011

\begin{tabular}{cccc}
\hline Rank $^{\mathrm{c}} /$ & Date of & & \\
annual & & & Peak \\
peaks & peak & Peak stage & stream- \\
in & flow & $(\mathrm{ft})$ & $\begin{array}{c}\text { streamflow } \\
\left(\mathrm{ft}^{3} / \mathrm{s}\right)\end{array}$ \\
record & . & &
\end{tabular}

\begin{tabular}{|c|c|c|c|c|c|c|c|c|c|c|}
\hline \multicolumn{11}{|c|}{ Missouri River Basin-Continued } \\
\hline 185 & 06109500 & $\begin{array}{l}\text { Missouri River at Virgelle, } \\
\text { Montana }\end{array}$ & 34,379 & $06 / 1953$ & -- & $122,000^{\mathrm{j}, \mathrm{d}}$ & $6 / 77$ & $6 / 12 / 2011$ & 14.07 & $53,700^{\mathrm{d}}$ \\
\hline 186 & 06112800 & $\begin{array}{l}\text { Bull Creek Tributary near Hilger, } \\
\text { Montana }\end{array}$ & 1.0 & $06 / 1991$ & 5.69 & 415 & $2 / 38$ & $5 / 21 / 2011$ & 5.16 & 114 \\
\hline 187 & 06114550 & $\begin{array}{l}\text { Wolf Creek Tributary near Cof- } \\
\text { fee Creek, Montana }\end{array}$ & 1.7 & $07 / 1978$ & 5.55 & 780 & $4 / 38$ & $6 / 9 / 2011$ & 6.05 & 190 \\
\hline 188 & 06114700 & $\begin{array}{l}\text { Judith River near mouth, Near } \\
\text { Winifred, Montana }\end{array}$ & 2,731 & $03 / 2003$ & $9.06^{\mathrm{g}}$ & 7,600 & $1 / 11$ & $5 / 26 / 2011$ & 10.46 & 15,300 \\
\hline 189 & 06115200 & $\begin{array}{l}\text { Missouri River near Landusky, } \\
\text { Montana }\end{array}$ & 40,987 & $06 / 1953$ & -- & $137,000^{\mathrm{d}}$ & $6 / 78$ & $6 / 11 / 2011$ & 32.35 & $74,800^{d}$ \\
\hline 190 & 06120500 & $\begin{array}{l}\text { Musselshell River at Harlowton, } \\
\text { Montana }\end{array}$ & 1,125 & $06 / 1975$ & 10.01 & 7,270 & $2 / 103$ & $5 / 25 / 2011$ & 10.25 & 5,520 \\
\hline 191 & 06123030 & $\begin{array}{l}\text { Musselshell River above Mud } \\
\text { Creek near Shawmut, Mon- } \\
\text { tana }\end{array}$ & 1,513 & $06 / 2010$ & 5.76 & 1,290 & $1 / 14$ & $5 / 26 / 2011$ & 9.27 & 8,900 \\
\hline 192 & 06126050 & $\begin{array}{l}\text { Musselshell River near Lavina, } \\
\text { Montana }\end{array}$ & 2,970 & 06/1997 & 11.13 & $6,220^{\mathrm{d}}$ & $1 / 20$ & $5 / 25 / 2011$ & 13.95 & $14,500^{\mathrm{d}}$ \\
\hline 193 & 06126500 & $\begin{array}{l}\text { Musselshell River near Roundup, } \\
\text { Montana }\end{array}$ & 4,023 & $06 / 1967$ & 12.45 & $9,610^{d}$ & $1 / 65$ & $5 / 26 / 2011$ & 14.78 & $15,000^{\mathrm{d}}$ \\
\hline 194 & 06127500 & $\begin{array}{l}\text { Musselshell River at Mus- } \\
\text { selshell, Montana }\end{array}$ & 4,568 & $06 / 1967$ & 11.57 & $9,850^{\mathrm{d}}$ & $1 / 66$ & $5 / 27 / 2011$ & 13.23 & $16,200^{\mathrm{d}}$ \\
\hline 195 & 06127520 & $\begin{array}{l}\text { Home Creek near Sumatra, } \\
\text { Montana }\end{array}$ & 2.0 & 03/1994 & 5.11 & 278 & $2 / 39$ & $5 / 10 / 2011$ & 5.10 & 164 \\
\hline 196 & 06127570 & $\begin{array}{l}\text { Butts Coulee near Melstone, } \\
\text { Montana }\end{array}$ & 6.7 & 07/1993 & 20.18 & 705 & $4 / 49$ & $5 / 10 / 2011$ & 14.53 & 402 \\
\hline 197 & 06127585 & $\begin{array}{l}\text { Little Wall Creek Tributary near } \\
\text { Flatwillow, Montana }\end{array}$ & 9.8 & 07/2009 & 6.78 & 45 & $1 / 38$ & $6 / 7 / 2011$ & 8.54 & 326 \\
\hline 198 & 06129000 & $\begin{array}{l}\text { Box Elder Creek near Winnett, } \\
\text { Montana }\end{array}$ & 684 & $06 / 1962$ & 15.34 & 9,910 & $2 / 22$ & $5 / 22 / 2011$ & $--^{y}$ & $9,450^{\mathrm{m}}$ \\
\hline 199 & 06130500 & $\begin{array}{l}\text { Musselshell River at Mosby, } \\
\text { Montana }\end{array}$ & 7,846 & $06 / 1944$ & -- & 18,000 & $1 / 80$ & $5 / 23 / 2011$ & 15.98 & 25,100 \\
\hline 200 & 06130915 & $\begin{array}{l}\text { Russian Coulee near Jordan, } \\
\text { Montana }\end{array}$ & 3.5 & 08/1993 & 7.45 & 840 & $3 / 38$ & $5 / 21 / 2011$ & 6.05 & 276 \\
\hline 201 & 06132000 & $\begin{array}{l}\text { Missouri River below Fort Peck } \\
\text { Dam, Montana }\end{array}$ & 57,556 & $08 / 1946$ & -- & $51,000^{\mathrm{d}}$ & $1 / 78$ & $6 / 15 / 2011$ & $15.53^{\mathrm{e}}$ & $65,900^{\mathrm{d}, 1}$ \\
\hline 202 & 06142400 & $\begin{array}{l}\text { Clear Creek near Chinook, } \\
\text { Montana }\end{array}$ & 135 & 09/1986 & 8.21 & 571 & $2 / 28$ & $6 / 4 / 2011$ & 8.44 & 571 \\
\hline 203 & 06144450 & $\begin{array}{l}\text { Middle Creek above Lodge } \\
\text { Creek near Govenlock, Sas- } \\
\text { katchewan }\end{array}$ & 276 & 09/1986 & 13.84 & 738 & $3 / 25$ & $4 / 27 / 2011$ & 9.89 & 494 \\
\hline 204 & 06154100 & $\begin{array}{l}\text { Milk River near Harlem, Mon- } \\
\text { tana }\end{array}$ & 9,822 & 1952 & -- & $19,000^{\mathrm{j}, \mathrm{d}, \mathrm{w}, \mathrm{m}}$ & $6 / 41$ & $6 / 11 / 2011$ & 23.46 & $6,380^{\mathrm{d}}$ \\
\hline 205 & 06155030 & $\begin{array}{l}\text { Milk River near Dodson, } \\
\text { Montana }\end{array}$ & 11,192 & 09/1986 & 29.79 & 13,200 & $2 / 29$ & $5 / 23 / 2011$ & 26.25 & 8,550 \\
\hline 206 & 06164510 & $\begin{array}{l}\text { Milk River at Juneberg Bridge } \\
\text { near Saco, Montana }\end{array}$ & 17,670 & $04 / 1978$ & 24.20 & $12,400^{\mathrm{d}}$ & $5 / 34$ & $4 / 14 / 2011$ & 22.07 & $10,300^{\mathrm{d}}$ \\
\hline
\end{tabular}




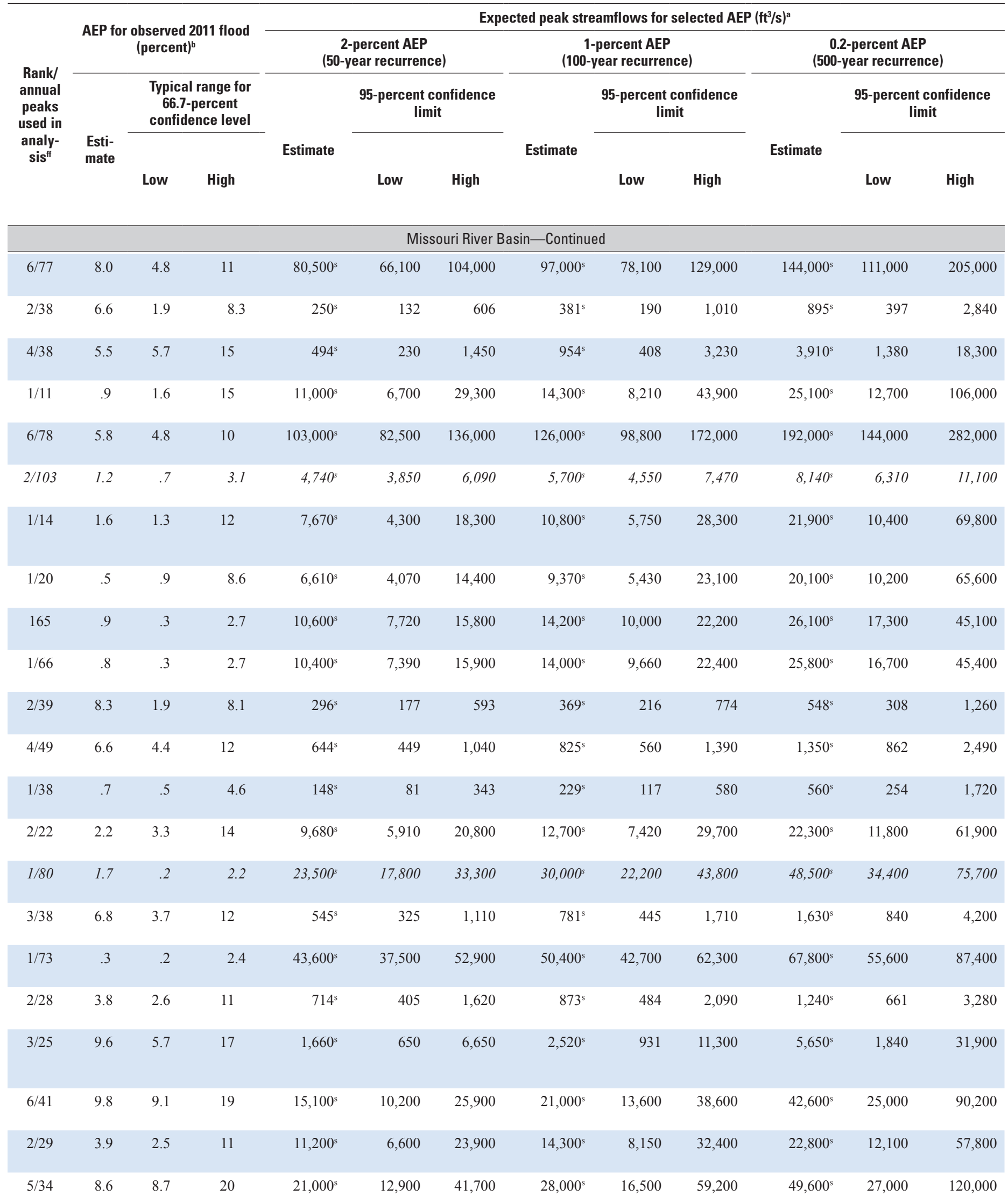




\section{Annual Exceedance Probabilities and Trends for the Central United States during the 2011 Floods}

Table 1. Peak streamflow data and results of flood-probability analyses for selected streamgages in the Central United States affected by 2011 flooding.-Continued

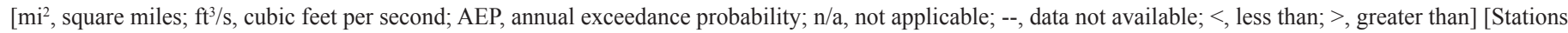
used in the trend analysis are italicized; Stations used in the trend analysis that overlap with trend analyses of Holmes and others (2010) are italicized and bold]

\section{Flood data}

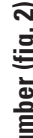

Contri-
buting
drainage
area
$\left(\mathrm{mi}^{2}\right)$

$\left(\mathrm{mi}^{2}\right)$
Previous maximum streamflow

Station name
Floods of 2011

number

area
$\left(\mathrm{mi}^{2}\right)$

\begin{tabular}{|c|c|c|c|}
\hline $\begin{array}{l}\text { Rank / } \\
\text { annual } \\
\text { peaks } \\
\text { in } \\
\text { record }\end{array}$ & $\begin{array}{c}\text { Date of } \\
\text { peak } \\
\text { stream- } \\
\text { flow }\end{array}$ & $\begin{array}{l}\text { Peak stage } \\
\text { (ft) }\end{array}$ & $\begin{array}{c}\text { Peak } \\
\text { streamflow } \\
\left(\mathrm{ft}^{3} / \mathbf{s}\right)\end{array}$ \\
\hline
\end{tabular}

Missouri River Basin-Continued

$\begin{array}{lll}207 & 06166000 & \begin{array}{c}\text { Beaver Creek below Guston } \\ \text { Coulee near Saco, Montana }\end{array}\end{array}$

20806167500 Beaver Creek near Hinsdale, Montana

\begin{tabular}{|c|c|c|c|c|c|c|c|c|c|c|}
\hline 209 & 06172310 & Milk River at Tampico, Montana & 21,078 & 03/1997 & -- & $11,000^{\mathrm{i}, 1}$ & $1 / 28$ & $4 / 15 / 2011$ & 28.03 & 19,700 \\
\hline 210 & 06174500 & Milk River at Nashua, Montana & 22,332 & $04 / 1952$ & 31.38 & $45,300^{\mathrm{d}}$ & $2 / 72$ & $6 / 9 / 2011$ & 29.52 & $26,500^{\mathrm{d}}$ \\
\hline 211 & 06177000 & $\begin{array}{l}\text { Missouri River near Wolf Point, } \\
\text { Montana }\end{array}$ & 82,290 & $03 / 1939$ & $14.40^{\mathrm{h}}$ & $66,800^{\mathrm{j}, \mathrm{d}}$ & $1 / 83$ & $6 / 14 / 2011$ & 14.77 & $93,200^{\mathrm{d}}$ \\
\hline 212 & 06178500 & $\begin{array}{l}\text { East Poplar River at interna- } \\
\text { tional boundary, Montana }\end{array}$ & 541 & $04 / 1975$ & 12.01 & 4,020 & $12 / 78$ & $4 / 12 / 2011$ & 11.41 & $1,830^{d}$ \\
\hline 213 & 06185500 & $\begin{array}{l}\text { Missouri River near Culbertson, } \\
\text { Montana }\end{array}$ & 91,557 & $03 / 1943$ & $15.12^{\mathrm{h}}$ & $78,200^{\mathrm{d}}$ & $1 / 63$ & $6 / 16 / 2011$ & 17.50 & $104,000^{\mathrm{d}}$ \\
\hline 214 & 06186500 & $\begin{array}{l}\text { Yellowstone River at Yellowstone } \\
\text { Lake Outlet, Wyoming }\end{array}$ & 991 & 06/1997 & 8.90 & 9,950 & $2 / 86$ & $7 / 11 / 2011$ & 8.74 & 9,560 \\
\hline 215 & 06187915 & $\begin{array}{l}\text { Soda Butte Creek at Park Bound- } \\
\text { ary at Silver Gate, Montana }\end{array}$ & 31.2 & $06 / 2010$ & 3.83 & 1,250 & $2 / 13$ & $6 / 30 / 2011$ & 3.85 & 1,230 \\
\hline 216 & 06188000 & $\begin{array}{l}\text { Lamar River near Tower Ranger } \\
\text { Station, Wyoming }\end{array}$ & 660 & $06 / 1996$ & 12.15 & 19,500 & $4 / 72$ & $6 / 24 / 2011$ & 10.75 & 15,500 \\
\hline 217 & 06191500 & $\begin{array}{l}\text { Yellowstone River at Corwin } \\
\text { Springs, Montana }\end{array}$ & 2,619 & $06 / 1996$ & 10.92 & 32,200 & $5 / 105$ & $6 / 30 / 2011$ & 10.36 & 30,300 \\
\hline 218 & 06192500 & $\begin{array}{l}\text { Yellowstone River near Livings- } \\
\text { ton, Montana }\end{array}$ & 3,551 & 06/1997 & 10.72 & 38,000 & $1 / 87$ & $6 / 30 / 2011$ & 10.15 & 40,600 \\
\hline 219 & 06195600 & $\begin{array}{l}\text { Shields River near Livingston, } \\
\text { Montana }\end{array}$ & 852 & $06 / 1979$ & 6.80 & 5,600 & $2 / 33$ & $5 / 25 / 2011$ & 6.36 & 4,360 \\
\hline 220 & 06200000 & $\begin{array}{l}\text { Boulder River at Big Timber, } \\
\text { Montana }\end{array}$ & 523 & 06/1997 & 9.00 & 9,940 & $4 / 64$ & $6 / 30 / 2011$ & 8.04 & 9,370 \\
\hline 221 & 06205000 & $\begin{array}{l}\text { Stillwater River near Absarokee, } \\
\quad \text { Montana }\end{array}$ & 935 & $06 / 1967$ & 7.17 & 12,000 & $6 / 81$ & $6 / 30 / 2011$ & 6.86 & 9,950 \\
\hline 222 & 06207500 & $\begin{array}{l}\text { Clarks Fork Yellowstone River } \\
\text { near Belfry, Montana }\end{array}$ & 1,154 & $06 / 1981$ & 9.97 & 14,800 & $3 / 90$ & $7 / 5 / 2011$ & 8.68 & 12,500 \\
\hline 223 & 06208500 & $\begin{array}{l}\text { Clarks Fork Yellowstone River at } \\
\text { Edgar, Montana }\end{array}$ & 2,022 & $06 / 1997$ & 9.30 & 11,100 & $5 / 72$ & $7 / 6 / 2011$ & 9.27 & 10,700 \\
\hline 224 & 06209500 & $\begin{array}{l}\text { Rock Creek near Red Lodge, } \\
\text { Montana }\end{array}$ & 105 & $06 / 1957$ & 4.78 & 3,110 & $4 / 63$ & $6 / 29 / 2011$ & 7.78 & 2,040 \\
\hline 225 & 06211000 & $\begin{array}{l}\text { Red Lodge Creek above Cooney } \\
\text { Reservoir near Boyd, Mon- } \\
\text { tana }\end{array}$ & 143 & $05 / 2005$ & 7.35 & 3,720 & $1 / 75$ & $5 / 25 / 2011$ & 7.74 & 4,700 \\
\hline 226 & 06211500 & $\begin{array}{l}\text { Willow Creek near Boyd, } \\
\text { Montana }\end{array}$ & 53.3 & $05 / 2005$ & 8.59 & 2,100 & $1 / 75$ & $5 / 25 / 2011$ & 8.60 & 2,110 \\
\hline 227 & 06214500 & $\begin{array}{l}\text { Yellowstone River at Billings, } \\
\text { Montana }\end{array}$ & 11,408 & 06/1997 & 15.00 & 82,000 & $3 / 86$ & $7 / 2 / 2011$ & 14.37 & 70,600 \\
\hline 228 & 06217300 & $\begin{array}{l}\text { Twelvemile Creek near Shep- } \\
\text { herd, Montana }\end{array}$ & 9.1 & $06 / 2001$ & 10.25 & 1,230 & $2 / 39$ & $5 / 24 / 2011$ & 4.23 & 395 \\
\hline 229 & 06218500 & $\begin{array}{l}\text { Wind River near Dubois, Wyo- } \\
\text { ming }\end{array}$ & 232 & $06 / 1972$ & $5.48^{\mathrm{g}}$ & $1,940^{\mathrm{d}}$ & $1 / 57$ & $7 / 1 / 2011$ & 5.65 & $2,040^{\mathrm{d}}$ \\
\hline
\end{tabular}




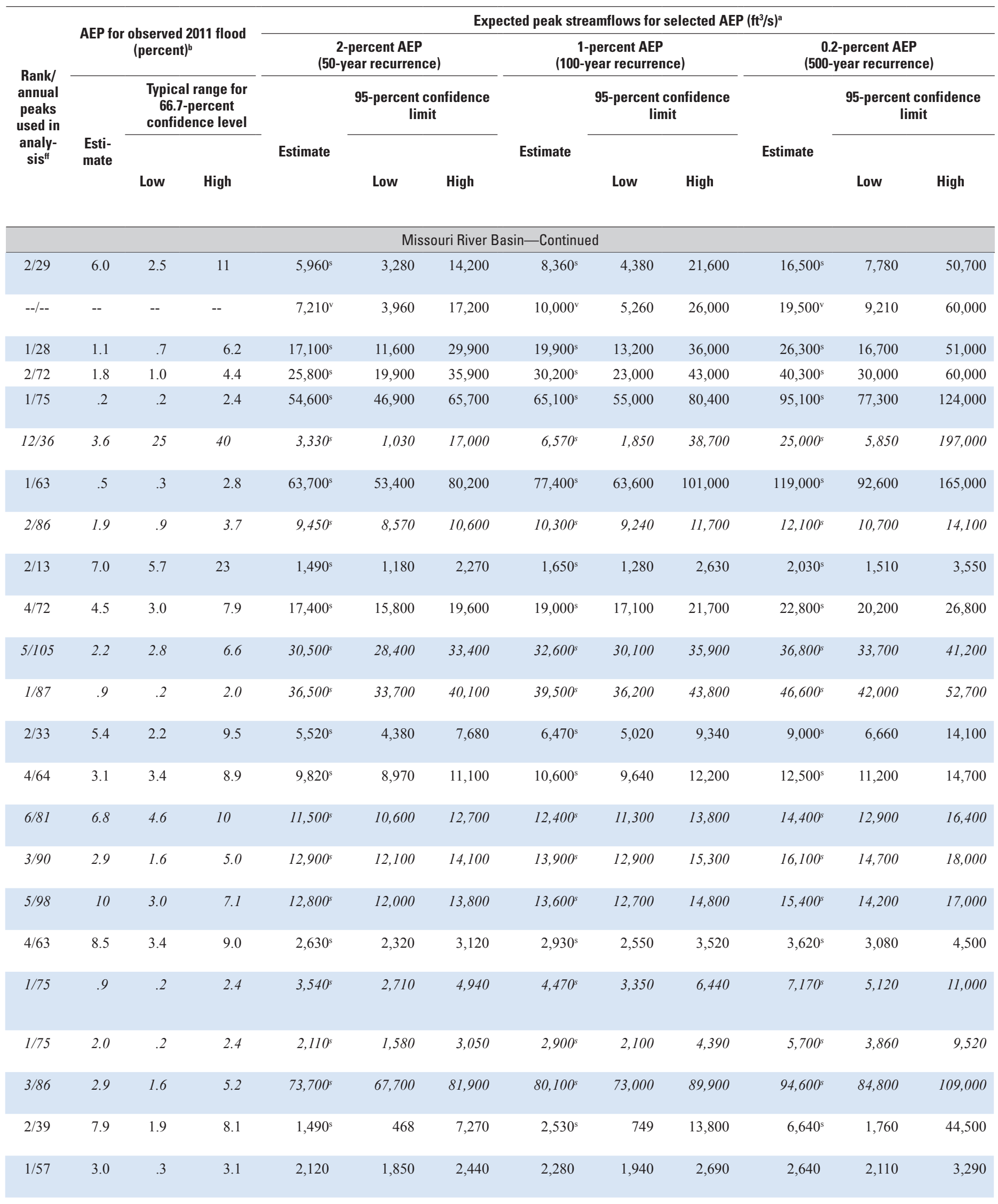




\section{Annual Exceedance Probabilities and Trends for the Central United States during the 2011 Floods}

Table 1. Peak streamflow data and results of flood-probability analyses for selected streamgages in the Central United States affected by 2011 flooding.-Continued

$\left[\mathrm{mi}^{2}\right.$, square miles; $\mathrm{ft}^{3} / \mathrm{s}$, cubic feet per second; AEP, annual exceedance probability; n/a, not applicable; --, data not available; <, less than; >, greater than] [Stations used in the trend analysis are italicized; Stations used in the trend analysis that overlap with trend analyses of Holmes and others (2010) are italicized and bold]

\begin{tabular}{|c|c|c|c|c|c|c|c|c|c|c|}
\hline \multirow{3}{*}{ 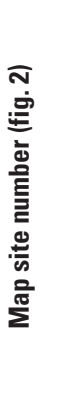 } & \multirow{3}{*}{$\begin{array}{c}\text { Site } \\
\text { number }\end{array}$} & \multirow{3}{*}{ Station name } & \multirow{3}{*}{$\begin{array}{l}\text { Contri- } \\
\text { buting } \\
\text { drainage } \\
\text { area } \\
\left(\mathrm{mi}^{2}\right)\end{array}$} & \multicolumn{7}{|c|}{ Flood data } \\
\hline & & & & \multicolumn{3}{|c|}{ Previous maximum streamflow } & \multicolumn{4}{|c|}{ Floods of 2011} \\
\hline & & & & $\begin{array}{l}\text { Date } \\
\text { (month/ } \\
\text { year) }\end{array}$ & Stage (ft) & $\begin{array}{l}\text { Streamflow } \\
\left(\mathrm{ft}^{3} / \mathrm{s}\right)\end{array}$ & $\begin{array}{l}\text { Rank }^{c} / \\
\text { annual } \\
\text { peaks } \\
\text { in } \\
\text { record }\end{array}$ & $\begin{array}{l}\text { Date of } \\
\text { peak } \\
\text { stream- } \\
\text { flow }\end{array}$ & $\begin{array}{l}\text { Peak stage } \\
\text { (ft) }\end{array}$ & $\begin{array}{l}\text { Peak } \\
\text { streamflow } \\
\left(\mathrm{ft}^{3} / \mathrm{s}\right)\end{array}$ \\
\hline \multicolumn{11}{|c|}{ Missouri River Basin—Continued } \\
\hline 230 & 06224000 & $\begin{array}{l}\text { Bull Lake Creek above Bull } \\
\text { Lake, Wyoming }\end{array}$ & 187 & 06/1981 & 7.98 & 4,470 & $2 / 58$ & $7 / 1 / 2011$ & 7.22 & 4,310 \\
\hline 231 & 06225500 & $\begin{array}{l}\text { Wind River near Crowheart, } \\
\text { Wyoming }\end{array}$ & 1,891 & 06/1991 & 11.04 & $14,300^{\mathrm{d}}$ & $1 / 67$ & $6 / 30 / 2011$ & 10.87 & $14,700^{\mathrm{d}}$ \\
\hline 232 & 06227600 & $\begin{array}{l}\text { Wind River near Kinnear, } \\
\text { Wyoming }\end{array}$ & 2,194 & 06/1991 & 8.03 & $13,900^{\mathrm{d}}$ & $1 / 27$ & $7 / 2 / 2011$ & $9.92^{\mathrm{j}}$ & $16,600^{\mathrm{j}, \mathrm{d}}$ \\
\hline 233 & 06228000 & $\begin{array}{l}\text { Wind River at Riverton, Wyo- } \\
\text { ming }\end{array}$ & 2,309 & $06 / 1935$ & $8.15^{\mathrm{f}}$ & $13,300^{\mathrm{d}}$ & $5 / 103$ & $7 / 2 / 2011$ & 11.80 & $11,200^{\mathrm{d}}$ \\
\hline 234 & 06236100 & $\begin{array}{l}\text { Wind River above Boysen Reser- } \\
\text { voir near Shoshoni, Wyoming }\end{array}$ & 4,390 & $06 / 2010$ & 9.19 & $19,200^{\mathrm{d}}$ & $2 / 21$ & $7 / 2 / 2011$ & 9.07 & $18,700^{\mathrm{d}}$ \\
\hline 235 & 06278300 & $\begin{array}{l}\text { Shell Creek above Shell Creek } \\
\text { Reservoir, Wyoming }\end{array}$ & 23.1 & $06 / 1963$ & $7.84^{i}$ & 1,870 & $2 / 55$ & $6 / 29 / 2011$ & 7.58 & 1,480 \\
\hline 236 & 06278500 & Shell Creek near Shell, Wyoming & 145 & 06/1945 & 7.49 & $3,020^{\mathrm{d}}$ & $1 / 70$ & $6 / 29 / 2011$ & 7.32 & $4,850^{\mathrm{d}}$ \\
\hline 237 & 06280300 & $\begin{array}{l}\text { South Fork Shoshone River near } \\
\text { Valley, Wyoming }\end{array}$ & 297 & 06/1981 & 9.24 & $10,000^{\mathrm{d}}$ & $2 / 54$ & $6 / 29 / 2011$ & 7.93 & $7,560^{\mathrm{d}}$ \\
\hline 238 & 06281000 & $\begin{array}{l}\text { South Fork Shoshone River } \\
\text { above Buffalo Bill Reservoir, } \\
\text { Wyoming }\end{array}$ & 585 & $06 / 1981$ & 9.41 & $9,960^{\mathrm{d}}$ & $4 / 48$ & $6 / 30 / 2011$ & 9.28 & $7,530^{\mathrm{d}}$ \\
\hline 240 & 06289820 & $\begin{array}{l}\text { East Pass Creek near Dayton, } \\
\text { Wyoming }\end{array}$ & 21.7 & $06 / 2007$ & $7.64^{\mathrm{f}}$ & $755^{\mathrm{d}}$ & $2 / 29$ & $5 / 21 / 2011$ & $9.05^{\mathrm{e}}$ & $732^{\mathrm{d}}$ \\
\hline 241 & 06291000 & $\begin{array}{l}\text { Owl Creek near Lodge Grass, } \\
\text { Montana }\end{array}$ & 163 & 06/1944 & 14.18 & 1,020 & $1 / 20$ & $5 / 25 / 2011$ & $--^{y}$ & $4,810^{\mathrm{m}}$ \\
\hline 242 & 06293300 & $\begin{array}{l}\text { Long Otter Creek near Lodge } \\
\text { Grass, Montana }\end{array}$ & 11.7 & $05 / 2008$ & 9.18 & 360 & $3 / 39$ & $5 / 22 / 2011$ & 5.96 & 192 \\
\hline 243 & 06294000 & $\begin{array}{l}\text { Little Bighorn River near Har- } \\
\quad \text { din, Montana }\end{array}$ & 1,294 & $05 / 1978$ & 11.20 & 22,600 & $2 / 59$ & $5 / 23 / 2011$ & 12.32 & 17,300 \\
\hline 244 & 06294400 & $\begin{array}{l}\text { Andresen Coulee near Custer, } \\
\text { Montana }\end{array}$ & 2.4 & 06/1991 & 4.40 & 255 & $3 / 49$ & $3 / 10 / 2011$ & 3.32 & 155 \\
\hline 245 & 06294500 & $\begin{array}{l}\text { Bighorn River above Tullock } \\
\text { Creek near Bighorn, Montana }\end{array}$ & 22,414 & 06/1991 & $7.17^{\mathrm{g}}$ & $16,100^{\mathrm{d}}$ & $1 / 47$ & $5 / 23 / 2011$ & 10.86 & $33,200^{\mathrm{d}}$ \\
\hline 246 & 06294930 & $\begin{array}{l}\text { Sarpy Creek Tributary near } \\
\text { Colstrip, Montana }\end{array}$ & 4.4 & 03/1978 & 4.84 & 590 & $5 / 40$ & $5 / 21 / 2011$ & 6.29 & 120 \\
\hline 247 & 06294985 & $\begin{array}{l}\text { East Fork Armells Creek Tribu- } \\
\text { tary near Colstrip, Montana }\end{array}$ & 1.9 & 07/1993 & 3.71 & 357 & $2 / 39$ & $5 / 21 / 2011$ & 3.44 & 291 \\
\hline 248 & 06295000 & $\begin{array}{l}\text { Yellowstone River at Forsyth, } \\
\text { Montana }\end{array}$ & 39,455 & 05/1978 & 14.53 & 109,800 & $2 / 37$ & $5 / 24 / 2011$ & 12.24 & 78,800 \\
\hline 249 & 06295113 & $\begin{array}{l}\text { Rosebud Creek at Reserva- } \\
\text { tion Boundary near Kirby, } \\
\text { Montana }\end{array}$ & 123 & 03/1996 & $6.30^{\mathrm{g}}$ & 219 & $1 / 32$ & $5 / 22 / 2011$ & 10.71 & 1,720 \\
\hline 250 & 06307616 & $\begin{array}{l}\text { Tongue River at Birney Day } \\
\text { School Bridge near Birney, } \\
\text { Montana }\end{array}$ & 2,621 & $06 / 2007$ & 7.06 & $5,340^{\mathrm{d}}$ & $3 / 32$ & $6 / 12 / 2011$ & 7.30 & $4,430^{\mathrm{d}}$ \\
\hline 251 & 06307740 & Otter Creek at Ashland, Montana & 707 & 03/1978 & $8.65^{\mathrm{h}}$ & 425 & $3 / 29$ & $5 / 25 / 2011$ & 5.51 & 347 \\
\hline 252 & 06307990 & $\begin{array}{l}\text { Tongue River above T\&Y Diver- } \\
\text { sion Dam near Miles City, } \\
\text { Montana }\end{array}$ & 4,508 & $06 / 2007$ & 10.40 & $7,510^{\mathrm{d}}$ & $2 / 7$ & $5 / 22 / 2011$ & 9.50 & $6,280^{\mathrm{d}}$ \\
\hline
\end{tabular}




\begin{tabular}{|c|c|c|c|c|c|c|c|c|c|c|c|c|}
\hline \multirow{4}{*}{$\begin{array}{c}\text { Rank/ } \\
\text { annual } \\
\text { peaks } \\
\text { used in } \\
\text { analy- } \\
\text { sis }^{f f}\end{array}$} & \multirow{2}{*}{\multicolumn{3}{|c|}{$\begin{array}{c}\text { AEP for observed } 2011 \text { flood } \\
\text { (percent) }^{\mathrm{b}}\end{array}$}} & \multicolumn{9}{|c|}{ Expected peak streamflows for selected AEP $\left(\mathrm{ft}^{3} / \mathrm{s}\right)^{\mathrm{a}}$} \\
\hline & & & & \multicolumn{3}{|c|}{$\begin{array}{c}\text { 2-percent AEP } \\
\text { (50-year recurrence) }\end{array}$} & \multicolumn{3}{|c|}{$\begin{array}{c}\text { 1-percent AEP } \\
\text { (100-year recurrence) }\end{array}$} & \multicolumn{3}{|c|}{$\begin{array}{c}\text { 0.2-percent AEP } \\
\text { (500-year recurrence) }\end{array}$} \\
\hline & \multirow{2}{*}{$\begin{array}{l}\text { Esti- } \\
\text { mate }\end{array}$} & \multicolumn{2}{|c|}{$\begin{array}{c}\text { Typical range for } \\
66.7-p e r c e n t \\
\text { confidence level }\end{array}$} & \multirow{2}{*}{ Estimate } & \multicolumn{2}{|c|}{$\begin{array}{c}\text { 95-percent confidence } \\
\text { limit }\end{array}$} & \multirow{2}{*}{ Estimate } & \multicolumn{2}{|c|}{$\begin{array}{l}\text { 95-percent confidence } \\
\text { limit }\end{array}$} & \multirow{2}{*}{ Estimate } & \multicolumn{2}{|c|}{$\begin{array}{c}\text { 95-percent confidence } \\
\text { limit }\end{array}$} \\
\hline & & Low & High & & Low & High & & Low & High & & Low & High \\
\hline \multicolumn{13}{|c|}{ Missouri River Basin—Continued } \\
\hline $2 / 58$ & 1.6 & 1.3 & 5.5 & 4,180 & 3,550 & 4,930 & 4,540 & 3,750 & 5,510 & 5,350 & 4,120 & 6,940 \\
\hline $1 / 66$ & 3.1 & .3 & 2.7 & $15,600^{\mathrm{s}}$ & 13,800 & 18,400 & $17,300^{\mathrm{s}}$ & 15,100 & 20,600 & $21,200^{s}$ & 18,100 & 26,000 \\
\hline $1 / 27$ & 3.6 & .7 & 6.4 & $18,900^{\mathrm{s}}$ & 14,500 & 27,900 & $21,800^{\mathrm{s}}$ & 16,400 & 33,400 & $29,100^{s}$ & 20,900 & 48,000 \\
\hline $1 / 61$ & 3.7 & .3 & 2.9 & $12,300^{\mathrm{s}}$ & 10,500 & 15,000 & $13,500^{\mathrm{s}}$ & 11,400 & 16,800 & $16,200^{\mathrm{s}}$ & 13,400 & 20,700 \\
\hline $2 / 21$ & 9.3 & 3.5 & 15 & $28,500^{s}$ & 20,400 & 47,800 & $33,300^{s}$ & 23,200 & 58,700 & $45,400^{\mathrm{s}}$ & 30,000 & 88,300 \\
\hline 255 & 1.5 & 1.3 & 5.8 & 1,410 & 1,170 & 1,710 & 1,550 & 1,240 & 1,930 & 1,850 & 1,370 & 2,510 \\
\hline$--/--$ & -- & -- & -- & $--^{z}$ & -- & -- & $--^{z}$ & -- & -- & $--^{2}$ & -- & -- \\
\hline $2 / 54$ & 1.9 & 1.4 & 5.9 & 7,510 & 6,220 & 9,070 & 8,190 & 6,570 & 10,200 & 9,730 & 7,210 & 13,100 \\
\hline $4 / 48$ & 6.3 & 4.5 & 12 & $9,260^{\mathrm{s}}$ & 7,980 & 11,300 & $10,400^{s}$ & 8,860 & 13,000 & $13,300^{\mathrm{s}}$ & 11,000 & 17,400 \\
\hline $2 / 29$ & 2.8 & 2.5 & 11 & $848^{s}$ & 525 & 1,710 & $1,190^{\mathrm{s}}$ & 699 & 2,620 & $2,430^{\mathrm{s}}$ & 1,280 & 6,440 \\
\hline $1 / 20$ & $<0.2$ & .9 & 8.6 & $1,580^{\mathrm{s}}$ & 966 & 3,470 & $2,160^{\mathrm{s}}$ & 1,250 & 5,290 & $4,240^{\mathrm{s}}$ & 2,180 & 13,200 \\
\hline $3 / 39$ & 8.2 & 3.6 & 11 & $363^{\mathrm{s}}$ & 210 & 763 & $462^{\mathrm{s}}$ & 261 & 1,020 & $715^{\mathrm{s}}$ & 384 & 1,740 \\
\hline $2 / 59$ & .3 & 1.2 & 5.4 & $8,350^{s}$ & 6,490 & 11,600 & $10,800^{\circ}$ & 8,140 & 15,700 & $18,500^{\circ}$ & 13,200 & 29,600 \\
\hline $3 / 49$ & 2.2 & 2.9 & 9.1 & $165^{\mathrm{s}}$ & 90 & 372 & $273^{\mathrm{s}}$ & 140 & 673 & $776^{\mathrm{s}}$ & 350 & 2,330 \\
\hline $1 / 47$ & 3.2 & .4 & 3.7 & $38,400^{s}$ & 29,500 & 54,600 & $47,800^{\mathrm{s}}$ & 35,800 & 70,800 & $75,800^{s}$ & 53,400 & 122,000 \\
\hline $5 / 40$ & 7.9 & 7.3 & 17 & $359^{\mathrm{s}}$ & 200 & 812 & $613^{s}$ & 319 & 1,560 & $1,950^{\mathrm{s}}$ & 872 & 6,450 \\
\hline $2 / 39$ & 7.0 & 1.9 & 8.1 & $570^{\mathrm{s}}$ & 302 & 1,360 & $777^{\mathrm{s}}$ & 397 & 1,970 & $1,380^{\mathrm{s}}$ & 661 & 3,970 \\
\hline $2 / 37$ & 6.2 & 2.0 & 8.5 & $95,100^{\mathrm{s}}$ & 82,300 & 117,000 & $106,000^{\mathrm{s}}$ & 90,700 & 134,000 & $135,000^{\mathrm{s}}$ & 111,000 & 179,000 \\
\hline $1 / 32$ & .3 & .6 & 5.4 & $614^{\mathrm{s}}$ & 372 & 1,270 & $880^{s}$ & 506 & 1,980 & $1,870^{\mathrm{s}}$ & 961 & 5,060 \\
\hline $3 / 32$ & 9.9 & 4.4 & 14 & $6,450^{\mathrm{s}}$ & 4,830 & 9,660 & $7,250^{\mathrm{s}}$ & 5,350 & 11,200 & $9,000^{\mathrm{s}}$ & 6,450 & 14,500 \\
\hline $3 / 29$ & 6.5 & 4.9 & 15 & $677^{\mathrm{s}}$ & 362 & 1,680 & $974^{\mathrm{s}}$ & 494 & 2,640 & $2,030^{\mathrm{s}}$ & 918 & 6,620 \\
\hline --/-- & -- & -- & -- & $8,080^{\mathrm{v}}$ & 6,100 & 12,400 & $9,320^{\mathrm{v}}$ & 6,880 & 14,900 & $12,400^{v}$ & 8,720 & 21,500 \\
\hline
\end{tabular}




\section{Annual Exceedance Probabilities and Trends for the Central United States during the 2011 Floods}

Table 1. Peak streamflow data and results of flood-probability analyses for selected streamgages in the Central United States affected by 2011 flooding.-Continued

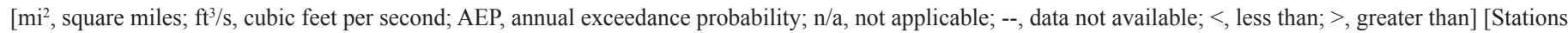
used in the trend analysis are italicized; Stations used in the trend analysis that overlap with trend analyses of Holmes and others (2010) are italicized and bold]

Flood data

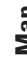

Contri-
buting
drainage
area
$\left(\mathrm{mi}^{2}\right)$

Previous maximum streamflow

Floods of 2011

number

Station name

$\left(\mathrm{mi}^{2}\right)$

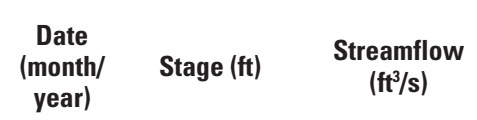

$\begin{array}{cc}\text { Rankc }^{\mathrm{c}} / & \text { Date of } \\ \text { annual } & \text { peak } \\ \text { peaks } & \text { stream- } \\ \text { in } & \text { flow } \\ \text { record } & \end{array}$

Peak stag

(ft)

Peak streamflow $\left(\mathrm{ft}^{3} / \mathrm{s}\right)$

\begin{tabular}{|c|c|c|c|c|c|c|c|c|c|c|}
\hline \multicolumn{11}{|c|}{ Missouri River Basin-Continued } \\
\hline 253 & 06308340 & $\begin{array}{l}\text { La Grange Creek near Volborg, } \\
\text { Montana }\end{array}$ & 3.7 & 07/1993 & 9.65 & 550 & $5 / 38$ & $5 / 23 / 2011$ & 8.50 & 208 \\
\hline 254 & 06308400 & $\begin{array}{l}\text { Pumpkin Creek near Miles City, } \\
\text { Montana }\end{array}$ & 697 & $05 / 1975$ & 12.27 & 2,890 & $1 / 20$ & $5 / 20 / 2011$ & 14.41 & 7,900 \\
\hline 255 & 06308500 & $\begin{array}{l}\text { Tongue River at Miles City, } \\
\text { Montana }\end{array}$ & 5,397 & 06/1962 & 11.33 & $13,300^{\mathrm{d}}$ & $1 / 70$ & $5 / 21 / 2011$ & 13.99 & $15,300^{\mathrm{d}}$ \\
\hline 256 & 06309000 & $\begin{array}{l}\text { Yellowstone River at Miles City, } \\
\text { Montana }\end{array}$ & 48,253 & 05/1978 & 16.50 & $102,000^{\mathrm{d}}$ & $3 / 84$ & $5 / 24 / 2011$ & 14.74 & $85,400^{\mathrm{d}}$ \\
\hline 257 & 06311000 & $\begin{array}{l}\text { North Fork Powder River near } \\
\text { Hazelton, Wyoming }\end{array}$ & 24.5 & $06 / 1953$ & $4.34^{f}$ & 886 & $3 / 65$ & $6 / 13 / 2011$ & 4.88 & 627 \\
\hline 258 & 06326500 & $\begin{array}{l}\text { Powder River near Locate, } \\
\quad \text { Montana }\end{array}$ & 13,068 & $02 / 1943$ & $11.23^{f}$ & 31,000 & $5 / 74$ & $5 / 21 / 2011$ & 11.70 & 24,100 \\
\hline 259 & 06327500 & $\begin{array}{l}\text { Yellowstone River at Glendive, } \\
\text { Montana }\end{array}$ & 66,739 & 06/1909 & 12.70 & 118,000 & $1 / 20$ & $5 / 23 / 2011$ & 56.37 & 125,000 \\
\hline 260 & 06329500 & $\begin{array}{l}\text { Yellowstone River near Sidney, } \\
\text { Montana }\end{array}$ & 68,392 & $06 / 1921$ & -- & 159,000 & $6 / 99$ & $5 / 24 / 2011$ & $22.02^{h, e}$ & 124,000 \\
\hline 261 & 06331000 & $\begin{array}{l}\text { Little Muddy River below Cow } \\
\text { Creek near Williston, North } \\
\text { Dakota }\end{array}$ & 775 & 04/1979 & 12.77 & $9,180^{\mathrm{d}}$ & $2 / 57$ & $4 / 12 / 2011$ & 12.37 & $7,470^{\mathrm{d}}$ \\
\hline 262 & 06332523 & $\begin{array}{l}\text { East Fork Shell Creek near } \\
\text { Parshall, North Dakota }\end{array}$ & 360 & 03/1999 & 6.39 & 1,170 & $1 / 20$ & $4 / 11 / 2011$ & 7.81 & 3,530 \\
\hline 263 & 06332770 & $\begin{array}{l}\text { Deepwater Creek at Mouth near } \\
\text { Raub, North Dakota }\end{array}$ & 220 & 03/1997 & $13.26^{\mathrm{h}}$ & $1,300^{\mathrm{j}}$ & $1 / 20$ & $4 / 11 / 2011$ & 16.10 & $5,180^{\mathrm{j}}$ \\
\hline 264 & 06334500 & $\begin{array}{l}\text { Little Missouri River at Camp } \\
\text { Crook, South Dakota }\end{array}$ & 1,974 & $04 / 2009$ & 17.67 & 12,400 & $1 / 56$ & $5 / 24 / 2011$ & 19.40 & 20,100 \\
\hline 265 & 06334625 & $\begin{array}{l}\text { Coal Creek tributary near Mill } \\
\text { Iron, Montana }\end{array}$ & .6 & 06/1991 & 6.64 & 205 & $4 / 38$ & $5 / 10 / 2011$ & 3.42 & 48 \\
\hline 266 & 06335500 & $\begin{array}{l}\text { Little Missouri River at Marm- } \\
\text { arth, North Dakota }\end{array}$ & 4,640 & $03 / 1947$ & 21.70 & 45,000 & $3 / 73$ & $5 / 23 / 2011$ & 21.11 & 40,300 \\
\hline 267 & 06336000 & $\begin{array}{l}\text { Little Missouri River at Medora, } \\
\text { North Dakota }\end{array}$ & 6,190 & 03/1947 & 20.50 & 65,000 & $5 / 61$ & $5 / 25 / 2011$ & 20.39 & 35,100 \\
\hline 268 & 06336600 & $\begin{array}{l}\text { Beaver Creek near Trotters, } \\
\text { North Dakota }\end{array}$ & 616 & 03/1978 & $18.61^{\mathrm{g}}$ & 2,720 & $2 / 34$ & 4/3/2011 & 18.50 & 2,560 \\
\hline 269 & 06337000 & $\begin{array}{l}\text { Little Missouri River near Wat- } \\
\quad \text { ford City, North Dakota }\end{array}$ & 8,310 & $03 / 1947$ & 24.00 & 110,000 & $5 / 77$ & $5 / 27 / 2011$ & 16.70 & 34,000 \\
\hline 271 & 06342500 & $\begin{array}{l}\text { Missouri River at Bismarck, } \\
\text { North Dakota }\end{array}$ & 186,400 & $04 / 1952$ & 27.90 & 500,000 & $8 / 83$ & $6 / 25 / 2011$ & $19.25^{\mathrm{e}}$ & $155,000^{d}$ \\
\hline 274 & 06357800 & $\begin{array}{l}\text { Grand River at Little Eagle, } \\
\text { South Dakota }\end{array}$ & 5,322 & 03/2009 & 24.60 & $32,400^{\mathrm{d}}$ & $5 / 53$ & $3 / 18 / 2011$ & $21.27^{\mathrm{h}, \mathrm{e}}$ & $16,000^{\mathrm{i}, \mathrm{d}}$ \\
\hline 275 & 06359500 & $\begin{array}{l}\text { Moreau River near Faith, South } \\
\text { Dakota }\end{array}$ & 2,596 & $04 / 1944$ & $21.90^{f}$ & 26,000 & $6 / 68$ & $3 / 17 / 2011$ & $19.92^{h, e}$ & $17,800^{j}$ \\
\hline 276 & 06360500 & $\begin{array}{l}\text { Moreau River near Whitehorse, } \\
\text { South Dakota }\end{array}$ & 4,878 & 03/1997 & $26.93^{g}$ & 29,700 & $1 / 57$ & $3 / 20 / 2011$ & 26.92 & 34,200 \\
\hline 277 & 06392900 & $\begin{array}{l}\text { Beaver Creek at Mallo Camp } \\
\text { near Four Corners, Wyoming }\end{array}$ & 10.3 & 04/1994 & 2.14 & 103 & $1 / 29$ & $5 / 21 / 2011$ & 2.37 & 154 \\
\hline
\end{tabular}




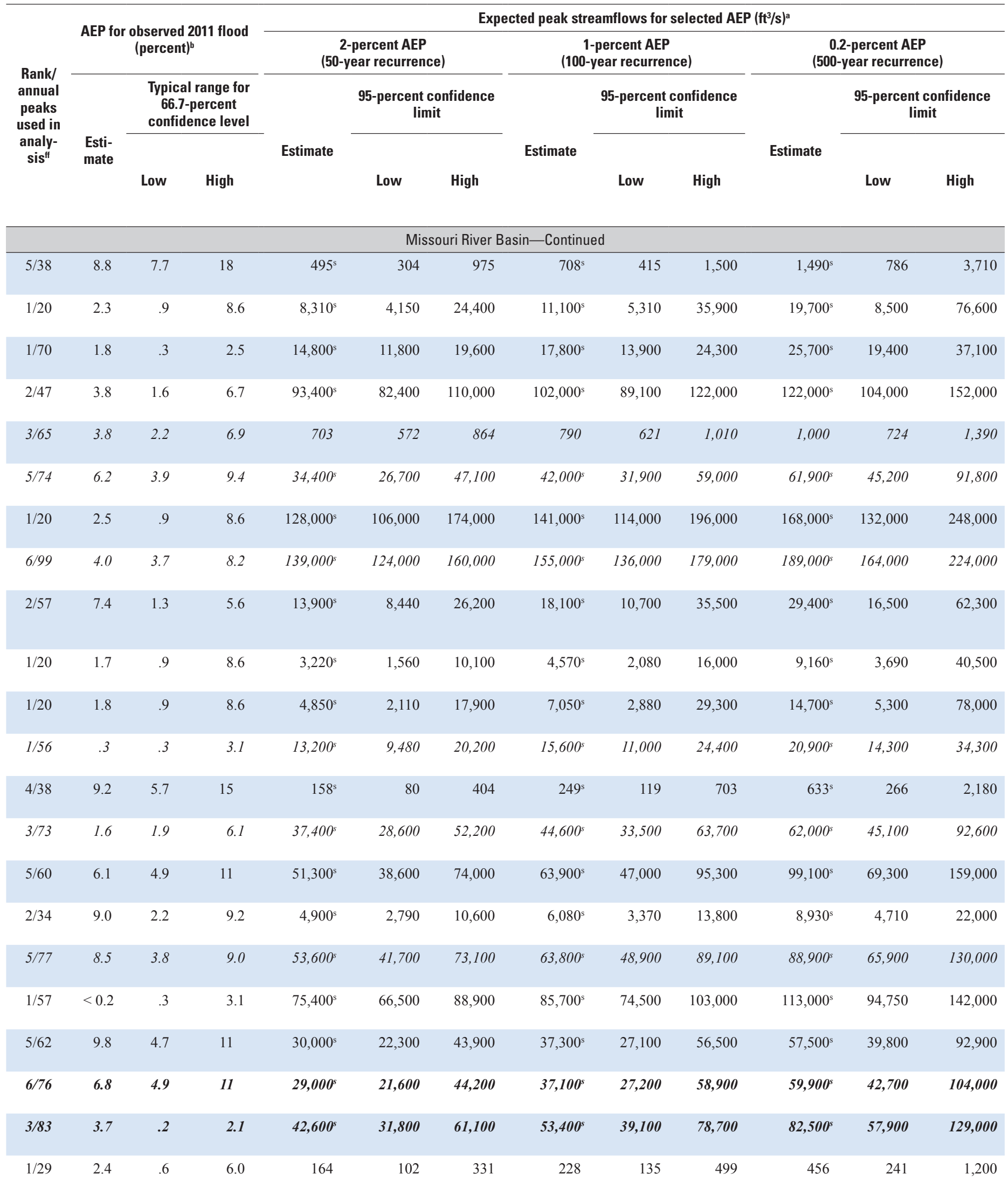




\section{Annual Exceedance Probabilities and Trends for the Central United States during the 2011 Floods}

Table 1. Peak streamflow data and results of flood-probability analyses for selected streamgages in the Central United States affected by 2011 flooding.-Continued

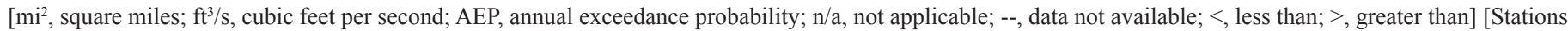
used in the trend analysis are italicized; Stations used in the trend analysis that overlap with trend analyses of Holmes and others (2010) are italicized and bold]

\begin{tabular}{|c|c|c|c|c|c|c|c|c|c|c|}
\hline \multirow{3}{*}{ 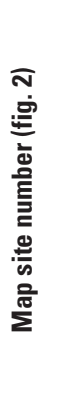 } & & \multirow{3}{*}{ Station name } & \multirow{3}{*}{$\begin{array}{l}\text { Contri- } \\
\text { buting } \\
\text { drainage } \\
\text { area } \\
\left(\mathrm{mi}^{2}\right)\end{array}$} & \multicolumn{7}{|c|}{ Flood data } \\
\hline & & & & \multicolumn{3}{|c|}{ Previous maximum streamflow } & \multicolumn{4}{|c|}{ Floods of 2011} \\
\hline & & & & $\begin{array}{l}\text { Date } \\
\text { (month/ } \\
\text { year) }\end{array}$ & Stage (ft) & $\begin{array}{l}\text { Streamflow } \\
\left(\mathrm{ft}^{3} / \mathrm{s}\right)\end{array}$ & $\begin{array}{l}\text { Rank }^{c} / \\
\text { annual } \\
\text { peaks } \\
\text { in } \\
\text { record }\end{array}$ & $\begin{array}{c}\text { Date of } \\
\text { peak } \\
\text { stream- } \\
\text { flow }\end{array}$ & $\begin{array}{l}\text { Peak stage } \\
\text { (ft) }\end{array}$ & $\begin{array}{c}\text { Peak } \\
\text { streamflow } \\
\left(\mathrm{ft}^{3} / \mathrm{s}\right)\end{array}$ \\
\hline \multicolumn{11}{|c|}{ Missouri River Basin-Continued } \\
\hline 278 & 06428500 & $\begin{array}{l}\text { Belle Fourche River at the } \\
\text { Wyoming-South Dakota State } \\
\text { Line }\end{array}$ & 3,241 & $05 / 1995$ & 16.33 & $6,320^{\mathrm{d}}$ & $2 / 65$ & $5 / 23 / 2011$ & 16.06 & $5,840^{\mathrm{d}}$ \\
\hline 279 & 06430800 & $\begin{array}{l}\text { Annie Creek near Lead, South } \\
\text { Dakota }\end{array}$ & 3.7 & $05 / 1995$ & 6.12 & 270 & $4 / 23$ & $5 / 22 / 2011$ & 5.35 & 109 \\
\hline 280 & 06430850 & $\begin{array}{l}\text { Little Spearfish Creek near Lead, } \\
\text { South Dakota }\end{array}$ & 27.8 & 06/1999 & 5.79 & 90 & $2 / 23$ & $5 / 22 / 2011$ & 5.21 & 67 \\
\hline 281 & 06436000 & $\begin{array}{l}\text { Belle Fourche River near Fruit- } \\
\text { dale, South Dakota }\end{array}$ & 4,513 & $05 / 1982$ & 14.32 & $12,700^{\mathrm{d}}$ & $4 / 66$ & $5 / 24 / 2011$ & 13.84 & $10,400^{\mathrm{d}}$ \\
\hline 282 & 06436760 & $\begin{array}{l}\text { Horse Creek above Vale, South } \\
\text { Dakota }\end{array}$ & 461 & $05 / 1982$ & 24.80 & $17,700^{\mathrm{d}}$ & $3 / 31$ & $5 / 21 / 2011$ & 21.05 & $11,100^{\mathrm{d}}$ \\
\hline 283 & 06437000 & $\begin{array}{l}\text { Belle Fourche River near Sturgis, } \\
\text { South Dakota }\end{array}$ & 5,814 & 05/1982 & 19.10 & $36,400^{\mathrm{d}}$ & $3 / 66$ & $5 / 25 / 2011$ & 17.39 & $24,400^{\mathrm{d}}$ \\
\hline 284 & 06438000 & $\begin{array}{l}\text { Belle Fourche River near Elm } \\
\text { Springs, South Dakota }\end{array}$ & 7,022 & $06 / 2008$ & 19.73 & $47,500^{\mathrm{d}}$ & $6 / 82$ & $5 / 25 / 2011$ & 16.09 & $35,800^{\mathrm{d}}$ \\
\hline 286 & 06441500 & $\begin{array}{l}\text { Bad River near Fort Pierre, } \\
\quad \text { South Dakota }\end{array}$ & 3,147 & $07 / 1905$ & 32.90 & $70,000^{j, w, m}$ & $6 / 86$ & $6 / 21 / 2011$ & 27.27 & 23,300 \\
\hline 288 & 06452320 & $\begin{array}{l}\text { Platte Creek near Platte, South } \\
\text { Dakota }\end{array}$ & 747 & $05 / 1995$ & 11.29 & 2,600 & $1 / 23$ & $6 / 22 / 2011$ & $12.91^{\mathrm{e}}$ & 3,570 \\
\hline 290 & 06468170 & $\begin{array}{l}\text { James River near Grace City, } \\
\text { North Dakota }\end{array}$ & 410 & $04 / 2009$ & 17.74 & 7,910 & $1 / 43$ & $4 / 11 / 2011$ & 17.54 & 8,140 \\
\hline 291 & 06468250 & $\begin{array}{l}\text { James River above Arrowwood } \\
\text { Lake near Kensal, North } \\
\text { Dakota }\end{array}$ & 450 & $04 / 2009$ & $14.70^{\mathrm{g}, \mathrm{h}}$ & 8,470 & $2 / 26$ & $4 / 12 / 2011$ & 14.41 & 7,800 \\
\hline 292 & 06469400 & $\begin{array}{l}\text { Pipestem Creek near Pingree, } \\
\text { North Dakota }\end{array}$ & 260 & $04 / 2009$ & 13.15 & 9,200 & $2 / 38$ & 4/9/2011 & $12.33^{\mathrm{e}}$ & 3,680 \\
\hline 293 & 06470000 & $\begin{array}{l}\text { James River at Jamestown, North } \\
\text { Dakota }\end{array}$ & 1,170 & $05 / 1950$ & 15.82 & 6,390 & $5 / 77$ & $9 / 20 / 2011$ & 13.01 & $2,470^{\mathrm{d}}$ \\
\hline 294 & 06470500 & $\begin{array}{l}\text { James River at Lamoure, North } \\
\text { Dakota }\end{array}$ & 1,790 & $04 / 2009$ & 17.56 & $12,200^{\mathrm{d}}$ & $5 / 62$ & $7 / 1 / 2011$ & $14.48^{\mathrm{h}, \mathrm{e}}$ & $4,600^{\mathrm{d}}$ \\
\hline 295 & 06470800 & $\begin{array}{l}\text { Bear Creek near Oakes, North } \\
\text { Dakota }\end{array}$ & 102 & 04/2009 & $12.25^{\mathrm{g}}$ & 1,900 & $1 / 35$ & $4 / 6 / 2011$ & 12.90 & 2,570 \\
\hline 296 & 06470878 & $\begin{array}{l}\text { James River at North Dakota- } \\
\text { South Dakota State Line }\end{array}$ & 2,180 & $04 / 2009$ & 96.35 & $11,800^{\mathrm{d}}$ & $2 / 10$ & $4 / 12 / 2011$ & $95.63^{\mathrm{h}, \mathrm{e}}$ & $8,070^{\mathrm{d}}$ \\
\hline 297 & 06471000 & $\begin{array}{l}\text { James River at Columbia, South } \\
\text { Dakota }\end{array}$ & 4,961 & $04 / 2009$ & $19.73^{h}$ & $9,620^{\mathrm{d}}$ & $2 / 66$ & $7 / 17 / 2011$ & $19.79^{\mathrm{h}, \mathrm{e}}$ & $6,200^{\mathrm{d}}$ \\
\hline 298 & 06472000 & $\begin{array}{l}\text { James River near Stratford, } \\
\text { South Dakota }\end{array}$ & 7,647 & $04 / 2009$ & 19.98 & 9,910 & $2 / 37$ & $4 / 29 / 2011$ & $21.57^{\mathrm{h}, \mathrm{e}}$ & 9,460 \\
\hline 299 & 06473000 & $\begin{array}{l}\text { James River at Ashton, South } \\
\text { Dakota }\end{array}$ & 8,326 & $04 / 2009$ & $23.03^{g, h}$ & 9,500 & $1 / 66$ & $5 / 2 / 2011$ & $24.40^{h, e}$ & 9,520 \\
\hline 300 & 06475000 & $\begin{array}{l}\text { James River near Redfield, South } \\
\text { Dakota }\end{array}$ & 11,869 & $04 / 1997$ & 29.92 & 17,000 & $3 / 62$ & $4 / 27 / 2011$ & 28.09 & 12,700 \\
\hline 301 & 06476000 & $\begin{array}{l}\text { James River at Huron, South } \\
\text { Dakota }\end{array}$ & 13,743 & $04 / 1997$ & 21.28 & 23,400 & $2 / 72$ & $3 / 26 / 2011$ & $20.07^{h}$ & 19,900 \\
\hline 302 & 06477000 & James River near Forestburg, & 15,549 & $04 / 1997$ & 20.61 & 25,600 & $1 / 62$ & $3 / 25 / 2011$ & 20.27 & 28,400 \\
\hline
\end{tabular}




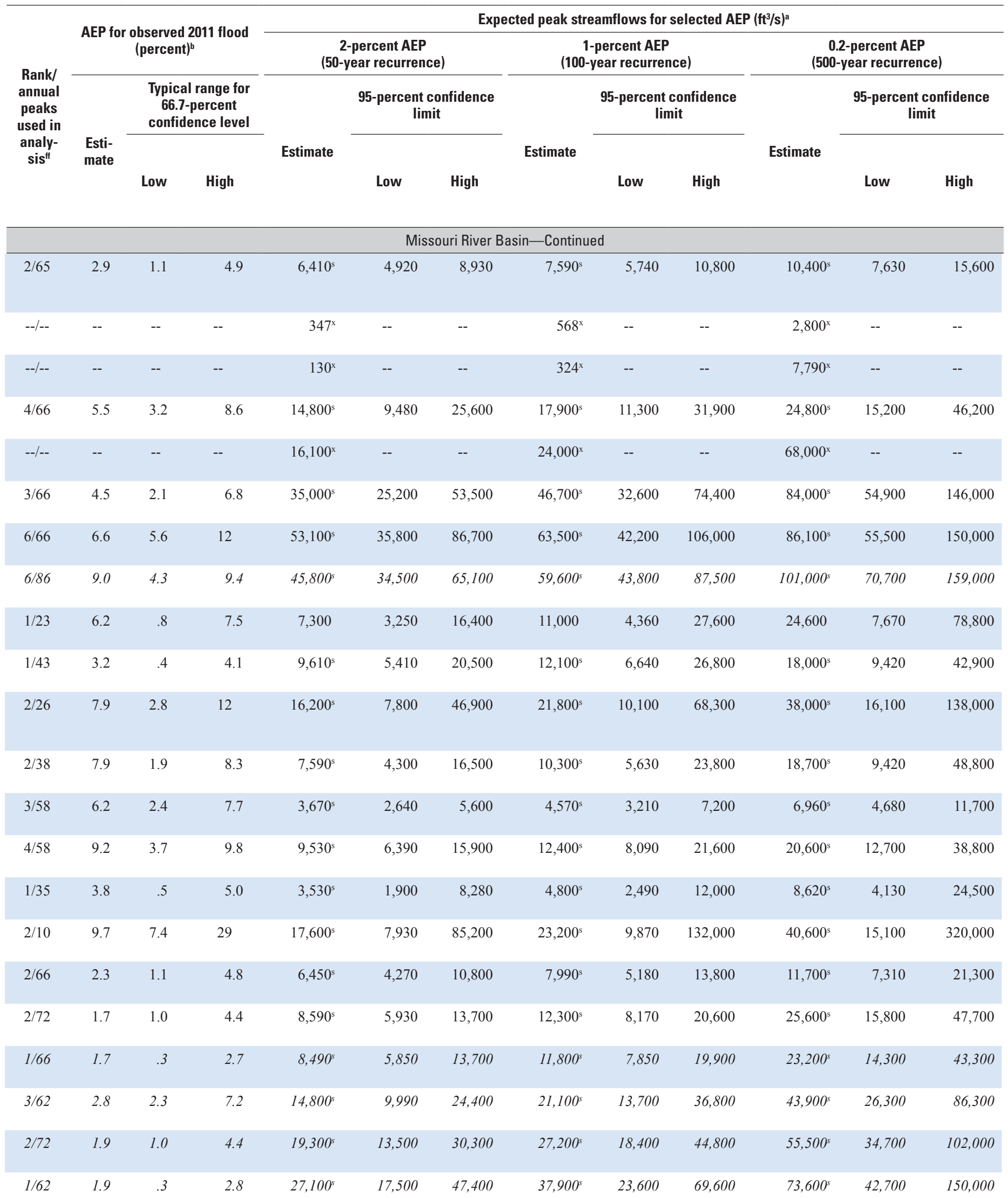




\section{Annual Exceedance Probabilities and Trends for the Central United States during the 2011 Floods}

Table 1. Peak streamflow data and results of flood-probability analyses for selected streamgages in the Central United States affected by 2011 flooding.-Continued

$\left[\mathrm{mi}^{2}\right.$, square miles; $\mathrm{ft}^{3} / \mathrm{s}$, cubic feet per second; AEP, annual exceedance probability; n/a, not applicable; --, data not available; <, less than; >, greater than] [Stations used in the trend analysis are italicized; Stations used in the trend analysis that overlap with trend analyses of Holmes and others (2010) are italicized and bold]

Flood data

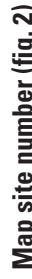

Contri-
buting
drainage
area
$\left(\mathrm{mi}^{2}\right)$

Site number
Station name
Previous maximum streamflow

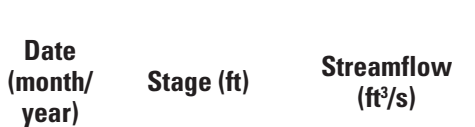

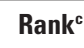

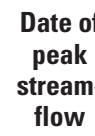

Floods of 2011

Peak stage Peak

(ft) streamflow

$\left(\mathrm{ft}^{3} / \mathbf{s}\right)$

\begin{tabular}{|c|c|c|c|c|c|c|c|c|c|c|}
\hline \multicolumn{11}{|c|}{ Missouri River Basin-Continued } \\
\hline 303 & 06477500 & $\begin{array}{l}\text { Firesteel Creek near Mount } \\
\text { Vernon, South Dakota }\end{array}$ & 587 & $07 / 2010$ & 16.04 & 7,420 & $6 / 56$ & $3 / 17 / 2011$ & $13.86^{e}$ & 4,720 \\
\hline 304 & 06478000 & $\begin{array}{l}\text { James River near Mitchell, South } \\
\text { Dakota }\end{array}$ & 17,023 & 04/1997 & $23.14^{\mathrm{f}}$ & $28,000^{\mathrm{m}}$ & $1 / 25$ & $3 / 25 / 2011$ & 25.20 & 28,400 \\
\hline 305 & 06478500 & $\begin{array}{l}\text { James River near Scotland, } \\
\quad \text { South Dakota }\end{array}$ & 18,601 & $06 / 1984$ & 20.45 & 29,400 & $2 / 83$ & $3 / 27 / 2011$ & 19.52 & 28,400 \\
\hline 306 & 06478513 & $\begin{array}{l}\text { James River near Yankton, South } \\
\text { Dakota }\end{array}$ & 18,891 & 04/1997 & 22.94 & 28,800 & $1 / 25$ & $3 / 28 / 2011$ & 22.24 & 29,200 \\
\hline 307 & 06480000 & $\begin{array}{l}\text { Big Sioux River near Brookings, } \\
\quad \text { South Dakota }\end{array}$ & 2,469 & $04 / 1969$ & 14.77 & 33,900 & $3 / 58$ & $3 / 24 / 2011$ & 13.33 & 15,400 \\
\hline 308 & 06481000 & $\begin{array}{l}\text { Big Sioux River near Dell Rap- } \\
\text { ids, South Dakota }\end{array}$ & 3,057 & $04 / 1969$ & 16.47 & 41,300 & $9 / 63$ & $3 / 24 / 2011$ & $15.63^{h}$ & 13,600 \\
\hline 309 & 06482020 & $\begin{array}{l}\text { Big Sioux River at North Cliff } \\
\text { Ave at Sioux Falls, South } \\
\text { Dakota }\end{array}$ & 3,778 & 04/1969 & 27.45 & $40,700^{\mathrm{d}, \mathrm{m}}$ & $5 / 41$ & $3 / 25 / 2011$ & 21.53 & $13,300^{\mathrm{d}}$ \\
\hline 311 & 06486000 & $\begin{array}{l}\text { Missouri River at Sioux City, } \\
\text { Iowa }\end{array}$ & 314,600 & $04 / 1952$ & $24.28^{\mathrm{f}}$ & 441,000 & $4 / 76$ & $7 / 20 / 2011$ & $35.25^{\mathrm{e}}$ & $192,000^{\mathrm{d}}$ \\
\hline 313 & 06601200 & $\begin{array}{l}\text { Missouri River at Decatur, } \\
\text { Nebraska }\end{array}$ & 316,200 & 04/1997 & 31.99 & $100,000^{\mathrm{d}}$ & $1 / 23$ & $6 / 28 / 2011$ & $40.24^{\mathrm{e}}$ & $191,000^{\mathrm{d}}$ \\
\hline 315 & 06610000 & $\begin{array}{l}\text { Missouri River at Omaha, } \\
\text { Nebraska }\end{array}$ & 322,800 & $04 / 1952$ & $30.20^{\mathrm{f}}$ & 396,000 & $2 / 83$ & $7 / 2 / 2011$ & 36.29 & $217,000^{\mathrm{d}}$ \\
\hline 316 & 06614800 & $\begin{array}{l}\text { Michigan River near Cameron } \\
\text { Pass, Colorado }\end{array}$ & 1.5 & 07/1995 & 3.69 & 115 & $4 / 38$ & 7/8/2011 & 3.80 & 90 \\
\hline 317 & 06622700 & $\begin{array}{l}\text { North Brush Creek near Sara- } \\
\text { toga, Wyoming }\end{array}$ & 37.4 & $06 / 2010$ & 5.38 & 1,360 & $1 / 52$ & $7 / 19 / 2011$ & 5.83 & 1,550 \\
\hline 318 & 06623800 & $\begin{array}{l}\text { Encampment River above Hog } \\
\text { Park Creek near Encampment, } \\
\text { Wyoming }\end{array}$ & 72.7 & $06 / 2010$ & 6.18 & 2,280 & $2 / 47$ & $6 / 30 / 2011$ & 6.06 & 2,180 \\
\hline 319 & 06630000 & $\begin{array}{l}\text { North Platte River above Semi- } \\
\text { noe Reservoir near Sinclair, } \\
\text { Wyoming }\end{array}$ & 4,061 & $06 / 2010$ & 11.64 & $16,700^{\mathrm{d}}$ & $2 / 72$ & 6/9/2011 & 11.46 & $16,200^{\mathrm{d}}$ \\
\hline 320 & 06657000 & $\begin{array}{l}\text { North Platte River below Whalen } \\
\text { Diversion Dam, Wyoming }\end{array}$ & 15,018 & $06 / 1955$ & $9.85^{\mathrm{f}}$ & $22,000^{\mathrm{d}}$ & $17 / 96$ & $6 / 22 / 2011$ & 10.31 & $7,210^{\mathrm{d}}$ \\
\hline 321 & 06674500 & $\begin{array}{l}\text { North Platte River at Wyoming- } \\
\text { Nebraska State Line }\end{array}$ & 22,218 & 06/1929 & $6.04^{\mathrm{f}}$ & $17,900^{\mathrm{d}}$ & $8 / 83$ & $6 / 11 / 2011$ & 6.45 & $8,050^{\mathrm{d}}$ \\
\hline 322 & 06715000 & $\begin{array}{l}\text { Clear Creek above West Fork } \\
\text { Clear Creek near Empire, } \\
\text { Colorado }\end{array}$ & 86.3 & $06 / 1995$ & 6.63 & 1,030 & $1 / 17$ & $7 / 8 / 2011$ & 6.46 & 1,060 \\
\hline 323 & 06727500 & $\begin{array}{l}\text { Fourmile Creek at Orodell, } \\
\text { Colorado }\end{array}$ & 24.2 & 06/1991 & $4.38^{\mathrm{f}}$ & 256 & $1 / 20$ & $7 / 13 / 2011$ & 9.80 & 770 \\
\hline 324 & 06746110 & $\begin{array}{l}\text { Joe Wright Creek below Joe } \\
\text { Wright Reservoir, Colorado }\end{array}$ & 6.9 & 08/1991 & 2.71 & $284^{\mathrm{d}}$ & $1 / 34$ & $6 / 30 / 2011$ & 2.83 & $301^{\mathrm{d}}$ \\
\hline 328 & 06807000 & $\begin{array}{l}\text { Missouri River at Nebraska City, } \\
\text { Nebraska }\end{array}$ & 410,000 & $04 / 1952$ & $24.48^{\mathrm{f,g}}$ & 414,000 & $2 / 82$ & 7/7/2011 & $28.27^{\mathrm{e}}$ & $229,000^{\mathrm{d}}$ \\
\hline 330 & 06813500 & $\begin{array}{l}\text { Missouri River at Rulo, Ne- } \\
\text { braska }\end{array}$ & 414,900 & $04 / 1952$ & 25.60 & 358,000 & $2 / 62$ & $6 / 27 / 2011$ & 27.26 & $328,000^{\mathrm{d}}$ \\
\hline
\end{tabular}




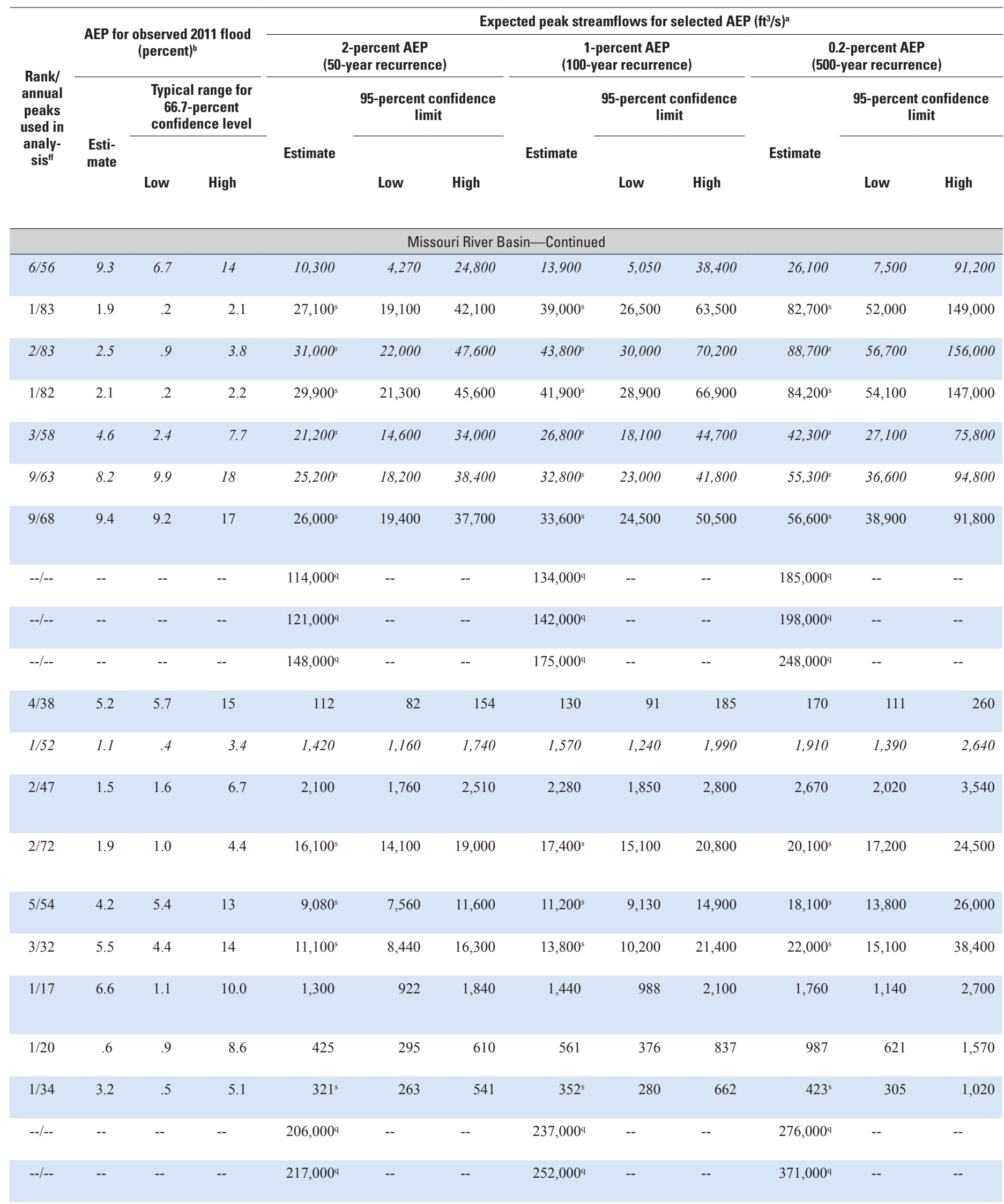




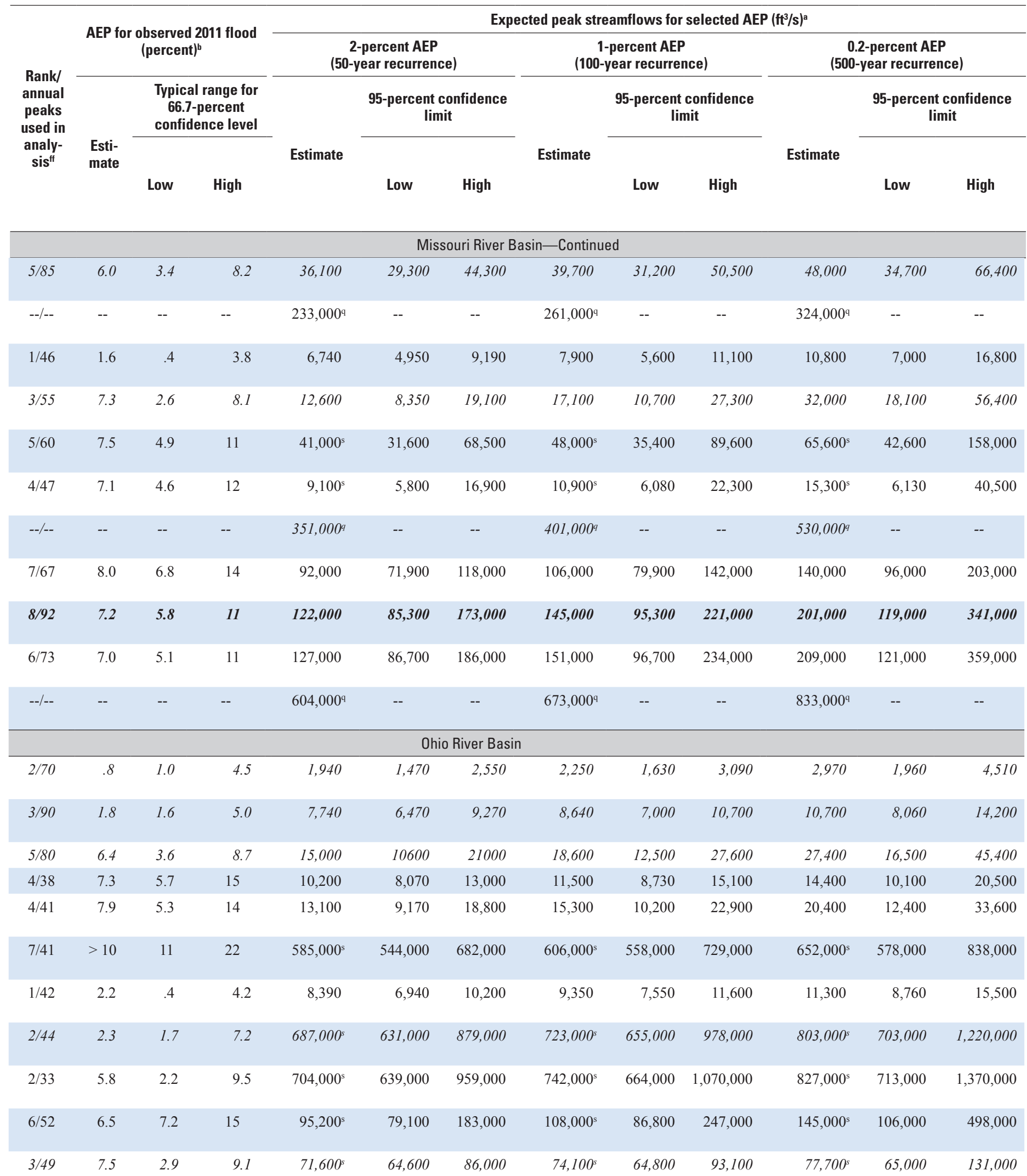




\section{$58 \quad$ Annual Exceedance Probabilities and Trends for the Central United States during the 2011 Floods}

Table 1. Peak streamflow data and results of flood-probability analyses for selected streamgages in the Central United States affected by 2011 flooding.-Continued

$\left[\mathrm{mi}^{2}\right.$, square miles; $\mathrm{ft}^{3} / \mathrm{s}$, cubic feet per second; AEP, annual exceedance probability; n/a, not applicable; --, data not available; <, less than; >, greater than] [Stations used in the trend analysis are italicized; Stations used in the trend analysis that overlap with trend analyses of Holmes and others (2010) are italicized and bold]

\begin{tabular}{|c|c|c|c|c|c|c|c|c|c|c|}
\hline \multirow{3}{*}{ 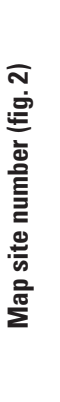 } & \multirow{3}{*}{$\begin{array}{l}\text { Site } \\
\text { number }\end{array}$} & \multirow{3}{*}{ Station name } & \multirow{3}{*}{$\begin{array}{l}\text { Contri- } \\
\text { buting } \\
\text { drainage } \\
\text { area } \\
\left(\mathrm{mi}^{2}\right)\end{array}$} & \multicolumn{7}{|c|}{ Flood data } \\
\hline & & & & \multicolumn{3}{|c|}{ Previous maximum streamflow } & \multicolumn{4}{|c|}{ Floods of 2011} \\
\hline & & & & $\begin{array}{c}\text { Date } \\
\text { (month/ } \\
\text { year) }\end{array}$ & Stage (ft) & $\begin{array}{l}\text { Streamflow } \\
\left(\mathrm{ft}^{3} / \mathrm{s}\right)\end{array}$ & $\begin{array}{l}\text { Rank }^{c} / \\
\text { annual } \\
\text { peaks } \\
\text { in } \\
\text { record }\end{array}$ & $\begin{array}{l}\text { Date of } \\
\text { peak } \\
\text { stream- } \\
\text { flow }\end{array}$ & $\begin{array}{l}\text { Peak stage } \\
\text { (ft) }\end{array}$ & $\begin{array}{c}\text { Peak } \\
\text { streamflow } \\
\left(\mathrm{ft}^{3} / \mathrm{s}\right)\end{array}$ \\
\hline \multicolumn{11}{|c|}{ Ohioi River Basin-Continued } \\
\hline 395 & 03374000 & $\begin{array}{l}\text { White River at Petersburg, } \\
\text { Indiana }\end{array}$ & 11,125 & 03/1913 & $29.50^{f}$ & $235,000^{j m}$ & $8 / 89$ & $5 / 3 / 2011$ & 26.88 & $133,000^{d}$ \\
\hline 396 & 03375500 & Patoka River at Jasper, Indiana & 262 & $03 / 1913$ & $15.90^{\mathrm{f}}$ & $16,000^{\mathrm{d}, \mathrm{w}}$ & $16 / 66$ & $5 / 3 / 2011$ & 16.68 & $4,550^{\mathrm{d}}$ \\
\hline 397 & 03376300 & Patoka River at Winslow, Indiana & 603 & $03 / 1964$ & $28.84^{g}$ & 15,500 & $5 / 38$ & $4 / 27 / 2011$ & 27.32 & $11,800^{\mathrm{d}}$ \\
\hline 398 & 03376500 & $\begin{array}{l}\text { Patoka River near Princeton, } \\
\text { Indiana }\end{array}$ & 822 & $01 / 1937$ & 26.80 & 18,700 & $2 / 77$ & $5 / 4 / 2011$ & 24.89 & $15,700^{\mathrm{d}}$ \\
\hline 399 & 03377500 & $\begin{array}{l}\text { Wabash River at Mount Carmel, } \\
\text { Illinois }\end{array}$ & 28,635 & $03 / 1913$ & $31.00^{f g}$ & 428,000 & $9 / 131$ & $5 / 3 / 2011$ & 34.02 & $270,000^{d}$ \\
\hline 400 & 03378550 & $\begin{array}{l}\text { Big Creek near Wadesville, } \\
\text { Indiana }\end{array}$ & 104 & $03 / 2008$ & 20.55 & 14,300 & $5 / 46$ & $4 / 24 / 2011$ & 19.64 & 9,240 \\
\hline 401 & 03380500 & $\begin{array}{l}\text { Skillet Fork at Wayne City, } \\
\text { Illinois }\end{array}$ & 464 & $05 / 1990$ & 25.75 & 59,400 & $8 / 94$ & $4 / 28 / 2011$ & 22.88 & 22,600 \\
\hline 402 & 03381500 & $\begin{array}{l}\text { Little Wabash River at Carmi, } \\
\text { Illinois }\end{array}$ & 3,102 & 05/1961 & $36.66^{g}$ & $46,900^{\mathrm{d}}$ & $1 / 72$ & $5 / 3 / 2011$ & $36.42^{\mathrm{e}}$ & $55,300^{\mathrm{d}}$ \\
\hline 403 & 03381700 & $\begin{array}{l}\text { Ohio River at Old Shawneetown, } \\
\text { Illinois }\end{array}$ & 141,000 & $01 / 2005$ & 50.57 & $1,030,000$ & $1 / 9$ & $5 / 6 / 2011$ & $56.37^{e}$ & $1,260,000$ \\
\hline 404 & 03382100 & $\begin{array}{l}\text { South Fork Saline River near } \\
\text { Carrier Mills, Illinois }\end{array}$ & 147 & $03 / 2008$ & 18.41 & 24,300 & $2 / 46$ & $5 / 3 / 2011$ & 16.11 & 7,870 \\
\hline 405 & 03383000 & $\begin{array}{l}\text { Tradewater River at Olney, } \\
\quad \text { Kentucky }\end{array}$ & 246 & $01 / 1937$ & 19.27 & $17,000^{w, m}$ & $4 / 70$ & $4 / 28 / 2011$ & 17.74 & 9,610 \\
\hline 406 & 03399800 & $\begin{array}{l}\text { Ohio River at Smithland Dam, } \\
\text { Smithland, Kentucky }\end{array}$ & 144,000 & 03/1997 & 51.44 & $831,000^{\mathrm{d}, 1}$ & $1 / 18$ & $5 / 6 / 2011$ & 54.83 & $1,170,000^{\mathrm{d}, 1}$ \\
\hline 415 & 03605555 & $\begin{array}{l}\text { Trace Creek above Denver, } \\
\text { Tennessee }\end{array}$ & 31.9 & $05 / 2010$ & 17.88 & $14,000^{\mathrm{j}, \mathrm{w}, \mathrm{m}, \mathrm{n}}$ & $4 / 49$ & $4 / 27 / 2011$ & 12.27 & 8,930 \\
\hline 416 & 03611500 & Ohio River at Metropolis, Illinois & 203,000 & $02 / 1937$ & -- & $1,850,000^{\mathrm{d}, 1}$ & $4 / 83$ & $5 / 6 / 2011$ & 61.65 & $1,280,000^{\mathrm{d}, \mathrm{l}}$ \\
\hline 417 & 03612000 & Cache River at Forman, Illinois & 244 & $03 / 2008$ & $39.01^{\mathrm{g}}$ & $20,400^{\mathrm{d}}$ & $2 / 88$ & $4 / 27 / 2011$ & 37.52 & $11,900^{\mathrm{d}}$ \\
\hline \multicolumn{11}{|c|}{ Lower Mississippi River Basin (downstream from the mouth of the Ohio River) } \\
\hline 418 & 07022300 & $\begin{array}{l}\text { Mississippi River near Wickliffe, } \\
\text { Kentucky }\end{array}$ & 918,500 & $02 / 1937$ & $58.18^{\mathrm{e}}$ & $2,010,000^{\text {aa }}$ & $1 / 1$ & $5 / 5 / 2011$ & $--^{y}$ & $2,070,000$ \\
\hline 419 & 07024500 & $\begin{array}{l}\text { South Fork Obion River near } \\
\text { Greenfield, Tennessee }\end{array}$ & 383 & $05 / 2010$ & $23.08^{\mathrm{g}}$ & 38,000 & $7 / 78$ & $5 / 3 / 2011$ & 21.76 & 18,200 \\
\hline 420 & 07026040 & $\begin{array}{l}\text { Obion River at Highway } 51 \text { near } \\
\text { Obion, Tennessee }\end{array}$ & 1,875 & $01 / 1937$ & 40.40 & $99,500^{\mathrm{m}}$ & $2 / 68$ & $5 / 6 / 2011$ & 39.31 & $63,900^{\mathrm{j}, 1}$ \\
\hline 421 & 07030050 & $\begin{array}{l}\text { Hatchie River at Rialto, Ten- } \\
\text { nessee }\end{array}$ & 2,308 & $01 / 1946$ & $22.90^{\mathrm{i}}$ & 55,700 & $1 / 47$ & $5 / 3 / 2011$ & 21.79 & 55,800 \\
\hline 422 & 07030240 & $\begin{array}{l}\text { Loosahatchie River near Arling- } \\
\text { ton, Tennessee }\end{array}$ & 262 & $05 / 2010$ & 25.44 & 29,600 & $5 / 42$ & $4 / 28 / 2011$ & 23.87 & 22,700 \\
\hline 423 & 07030392 & $\begin{array}{l}\text { Wolf River at La Grange, Ten- } \\
\text { nessee }\end{array}$ & 210 & $05 / 2010$ & 15.72 & 12,200 & $1 / 16$ & $4 / 27 / 2011$ & 15.88 & 12,800 \\
\hline 424 & 07030500 & $\begin{array}{l}\text { Wolf River at Rossville, Ten- } \\
\text { nessee }\end{array}$ & 503 & $01 / 1935$ & 13.75 & 40,000 & $3 / 52$ & $4 / 28 / 2011$ & 14.46 & 29,400 \\
\hline 425 & 07031650 & $\begin{array}{l}\text { Wolf River at Germantown, } \\
\text { Tennessee }\end{array}$ & 699 & 03/1975 & 27.98 & 33,400 & $2 / 38$ & $4 / 29 / 2011$ & 26.91 & 28,900 \\
\hline 426 & 07032000 & $\begin{array}{l}\text { Mississippi River at Memphis, } \\
\text { Tennessee }\end{array}$ & 932,800 & $02 / 1937$ & $50.40^{\mathrm{bb}, \mathrm{e}}$ & $2,020,000^{\mathrm{cc}}$ & $1 / 83$ & $5 / 9 / 2011$ & 48.03 & $2,190,000$ \\
\hline
\end{tabular}




\begin{tabular}{|c|c|c|c|c|c|c|c|c|c|c|c|c|}
\hline \multirow{4}{*}{$\begin{array}{l}\text { Rank/ } \\
\text { annual } \\
\text { peaks } \\
\text { used in } \\
\text { analy- } \\
\text { sisf }^{-1}\end{array}$} & \multirow{2}{*}{\multicolumn{3}{|c|}{$\begin{array}{c}\text { AEP for observed } 2011 \text { flood } \\
\text { (percent) })^{\mathrm{b}}\end{array}$}} & \multicolumn{9}{|c|}{ Expected peak streamflows for selected AEP $\left(\mathrm{ft}^{3} / \mathrm{s}\right)^{\mathrm{a}}$} \\
\hline & & & & \multicolumn{3}{|c|}{$\begin{array}{c}\text { 2-percent AEP } \\
\text { (50-year recurrence) }\end{array}$} & \multicolumn{3}{|c|}{$\begin{array}{c}\text { 1-percent AEP } \\
\text { (100-year recurrence) }\end{array}$} & \multicolumn{3}{|c|}{$\begin{array}{c}\text { 0.2-percent AEP } \\
\text { (500-year recurrence) }\end{array}$} \\
\hline & \multirow{2}{*}{$\begin{array}{l}\text { Esti- } \\
\text { mate }\end{array}$} & \multicolumn{2}{|c|}{$\begin{array}{c}\text { Typical range for } \\
66.7-p e r c e n t \\
\text { confidence level }\end{array}$} & \multirow{2}{*}{ Estimate } & \multicolumn{2}{|c|}{$\begin{array}{l}\text { 95-percent confidence } \\
\text { limit }\end{array}$} & \multirow{2}{*}{ Estimate } & \multicolumn{2}{|c|}{$\begin{array}{l}\text { 95-percent confidence } \\
\text { limit }\end{array}$} & \multirow{2}{*}{ Estimate } & \multicolumn{2}{|c|}{$\begin{array}{c}\text { 95-percent confidence } \\
\text { limit }\end{array}$} \\
\hline & & Low & High & & Low & High & & Low & High & & Low & High \\
\hline \multicolumn{13}{|c|}{ Ohio River Basin-Continued } \\
\hline $3 / 50$ & 5.7 & 2.8 & 8.9 & $152,000^{5}$ & 129,000 & 199,000 & $165,000^{5}$ & 133,000 & 224,000 & $192,000^{5}$ & 138,000 & 290,000 \\
\hline $3 / 34$ & 9.5 & 4.2 & 13 & $6,780^{\mathrm{s}}$ & 5,120 & 16,800 & $7,920^{\mathrm{s}}$ & 5,740 & 23,500 & $11,000^{\mathrm{s}}$ & 7,140 & 50,400 \\
\hline $3 / 25$ & 6.1 & 5.7 & 17 & $14,100^{\text {s }}$ & 9,720 & 22,600 & $15,600^{\mathrm{s}}$ & 9,720 & 26,300 & $18,500^{\mathrm{s}}$ & 9,720 & 37,600 \\
\hline $1 / 34$ & 2.7 & .5 & 5.1 & $16,600^{\mathrm{s}}$ & 12,600 & 35,200 & $19,000^{\mathrm{s}}$ & 13,900 & 47,900 & $25,100^{\mathrm{s}}$ & 16,300 & 91,900 \\
\hline $1 / 44$ & 2.1 & .4 & 4.0 & $271,000^{5}$ & 223,000 & 333,000 & $287,000^{s}$ & 223,000 & 365,000 & $319,000^{s}$ & 223,000 & 444,000 \\
\hline $5 / 46$ & 4.8 & 6.4 & 15 & 10,300 & 8,230 & 12,800 & 11,200 & 8,660 & 14,500 & 12,900 & 9,240 & 18,000 \\
\hline $8 / 94$ & 9.3 & 5.7 & 11 & 36,200 & 27,700 & 47,300 & 42,700 & 31,200 & 58,400 & 58,800 & 38,500 & 89,700 \\
\hline $1 / 72$ & .7 & .3 & 2.5 & $43,300^{\mathrm{s}}$ & 35,700 & 63,700 & $49,400^{\mathrm{s}}$ & 39,400 & 79,500 & $64,200^{\mathrm{s}}$ & 46,800 & 128,000 \\
\hline$--/--$ & -- & -- & -- & -- & -- & -- & -- & -- & -- & -- & -- & -- \\
\hline $2 / 46$ & 4.1 & 1.6 & 6.9 & 9,580 & 6,640 & 13,800 & 11,800 & 7,690 & 18,000 & 16,100 & 9,150 & 28,200 \\
\hline $4 / 70$ & 6.9 & 3.1 & 8.2 & 13,900 & 10,400 & 18,600 & 17,100 & 12,100 & 24,000 & 26,400 & 16,700 & 41,500 \\
\hline $1 / 18$ & $<0.2$ & 1.0 & 9.5 & $851,000^{\mathrm{s}}$ & 760,000 & $1,250,000$ & $887,000^{\mathrm{s}}$ & 780,000 & $1,370,000$ & $961,000^{\mathrm{s}}$ & 801,000 & $1,770,000$ \\
\hline $3 / 48$ & 4.1 & 2.9 & 9.2 & 10,600 & 7,640 & 14,800 & 12,400 & 8,440 & 18,100 & 16,700 & 10,200 & 27,500 \\
\hline $4 / 83$ & 4.0 & 2.6 & 6.9 & $1,360,000^{\mathrm{s}}$ & $1,260,000$ & $1,560,000$ & $1,430,000^{\mathrm{s}}$ & $1,310,000$ & $1,700,000$ & $1,590,000^{\mathrm{s}}$ & $1,400,000$ & $2,040,000$ \\
\hline $2 / 88$ & 2.4 & .8 & 3.6 & $12,400^{\mathrm{s}}$ & 9,960 & 18,500 & $14,600^{s}$ & 11,300 & 23,900 & $20,400^{\mathrm{s}}$ & 14,300 & 41,600 \\
\hline \multicolumn{13}{|c|}{ Lower Mississippi River Basin (downstream from the mouth of the Ohio River) } \\
\hline$--/--$ & -- & -- & -- & -- & -- & -- & -- & -- & -- & -- & -- & -- \\
\hline $7 / 78$ & 8.7 & 5.8 & 12 & 26,900 & 20,800 & 34,900 & 31,400 & 23,200 & 42,500 & 42,400 & 28,200 & 63,800 \\
\hline $2 / 68$ & 3.5 & 1.1 & 4.7 & 71,400 & 56,500 & 90,300 & 81,400 & 61,900 & 107,000 & 106,000 & 73,100 & 153,000 \\
\hline $1 / 47$ & 3.7 & .4 & 3.7 & 63,600 & 48,400 & 83,500 & 72,900 & 53,100 & 100,000 & 96,400 & 63,200 & 147,000 \\
\hline $5 / 42$ & 6.9 & 7.0 & 16 & 29,600 & 21,900 & 40,000 & 33,600 & 23,800 & 47,600 & 43,600 & 27,600 & 68,900 \\
\hline $1 / 16$ & 10 & 1.1 & 11 & 20,300 & 12,400 & 33,000 & 23,500 & 13,700 & 40,300 & 31,000 & 16,000 & 60,400 \\
\hline $3 / 52$ & 3.3 & 2.7 & 8.6 & 32,400 & 24,300 & 43,200 & 36,900 & 26,400 & 51,600 & 47,300 & 30,200 & 74,300 \\
\hline $2 / 38$ & 4.8 & 1.9 & 8.3 & 35,700 & 25,300 & 50,500 & 41,700 & 28,100 & 62,000 & 56,900 & 34,000 & 95,300 \\
\hline $1 / 81$ & .4 & .2 & 2.2 & $1,880,000^{\mathrm{dd}}$ & $1,730,000$ & $2,200,000$ & $1,990,000^{\mathrm{dd}}$ & $1,810,000$ & $2,430,000$ & $2,240,000^{\mathrm{dd}}$ & $1,960,000$ & $2,990,000$ \\
\hline
\end{tabular}




\section{Annual Exceedance Probabilities and Trends for the Central United States during the 2011 Floods}

Table 1. Peak streamflow data and results of flood-probability analyses for selected streamgages in the Central United States affected by 2011 flooding.-Continued

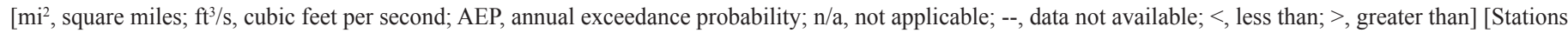
used in the trend analysis are italicized; Stations used in the trend analysis that overlap with trend analyses of Holmes and others (2010) are italicized and bold]

\section{Flood data}

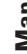

Contri-
buting
drainage
area
$\left(\mathrm{mi}^{2}\right)$

Previous maximum streamflow

Site number

Station name

Date
$\begin{gathered}\text { (month/ } \\ \text { year) }\end{gathered}$ Stage $(\mathbf{f t}) \quad \begin{gathered}\text { Streamflow } \\ \left(\mathrm{ft}^{3} / \mathbf{s}\right)\end{gathered}$

Floods of 2011

\begin{tabular}{|c|c|c|c|}
\hline \multicolumn{4}{|c|}{ Floods of 2011} \\
\hline $\begin{array}{l}\text { Rank }{ }^{c} / \\
\text { annual } \\
\text { peaks } \\
\text { in } \\
\text { record }\end{array}$ & $\begin{array}{l}\text { Date of } \\
\text { peak } \\
\text { stream- } \\
\text { flow }\end{array}$ & $\begin{array}{c}\text { Peak stage } \\
\text { (ft) }\end{array}$ & $\begin{array}{c}\text { Peak } \\
\text { streamflow } \\
\left(\mathbf{f t}^{3} / \mathbf{s}\right)\end{array}$ \\
\hline
\end{tabular}

\begin{tabular}{|c|c|c|c|c|c|c|c|c|c|c|}
\hline \multicolumn{11}{|c|}{ Lower Mississippi River Basin (downstream from the mouth of the Ohio River) - Continued } \\
\hline 427 & 07032200 & $\begin{array}{l}\text { Nonconnah Creek near German- } \\
\text { town, Tennessee }\end{array}$ & 68.2 & $10 / 2002$ & 23.87 & 14,600 & $2 / 42$ & $4 / 27 / 2011$ & 23.48 & 13,800 \\
\hline 428 & 07035000 & $\begin{array}{l}\text { Little St. Francis River at Freder- } \\
\text { icktown, Missouri }\end{array}$ & 90.5 & $11 / 1993$ & 26.50 & 25,100 & $2 / 23$ & $4 / 26 / 2011$ & 22.39 & 15,000 \\
\hline 429 & 07036100 & $\begin{array}{l}\text { St. Francis River near Saco, } \\
\text { Missouri }\end{array}$ & 664 & $11 / 1993$ & 36.10 & 161,000 & $4 / 20$ & $4 / 26 / 2011$ & 27.94 & 63,200 \\
\hline 430 & 07037500 & $\begin{array}{l}\text { St. Francis River near Patterson, } \\
\text { Missouri }\end{array}$ & 956 & $12 / 1982$ & 35.77 & 155,000 & $3 / 90$ & $5 / 2 / 2011$ & 30.92 & 80,000 \\
\hline 431 & 07039500 & $\begin{array}{l}\text { St. Francis River at Wappapello, } \\
\text { Missouri }\end{array}$ & 1,311 & 08/1915 & -- & $85,000^{\text {w }}$ & $2 / 71$ & $5 / 3 / 2011$ & 35.64 & $28,100^{\mathrm{d}}$ \\
\hline 432 & 07040000 & $\begin{array}{l}\text { St. Francis River at Fisk, Mis- } \\
\text { souri }\end{array}$ & 1,370 & $04 / 2008$ & 23.33 & $11,400^{\mathrm{d}}$ & $1 / 13$ & $5 / 3 / 2011$ & 27.10 & $18,800^{\mathrm{d}}$ \\
\hline 433 & 07040100 & $\begin{array}{l}\text { St. Francis River at St. Francis, } \\
\text { Arkansas }\end{array}$ & 1,770 & $03 / 1935$ & 28.20 & 39,200 & $2 / 90$ & $5 / 3 / 2011$ & $27.25^{\mathrm{e}}$ & $27,000^{\mathrm{d}}$ \\
\hline 434 & 07040450 & $\begin{array}{l}\text { St. Francis River at Lake City, } \\
\text { Arkansas }\end{array}$ & 2,370 & 04/1979 & -- & $42,700^{\mathrm{d}}$ & $2 / 87$ & $5 / 3 / 2011$ & 14.37 & $42,600^{\mathrm{d}}$ \\
\hline 435 & 07043500 & $\begin{array}{l}\text { Little River Ditch } 1 \text { near More- } \\
\text { house, Missouri }\end{array}$ & 341 & 02/1989 & 19.50 & 12,200 & $3 / 63$ & $4 / 28 / 2011$ & 19.77 & 10,300 \\
\hline 436 & 07047800 & $\begin{array}{l}\text { St. Francis River at Parkin, } \\
\text { Arkansas }\end{array}$ & $--^{k}$ & $01 / 1930$ & -- & $25,300^{\mathrm{d}}$ & $5 / 75$ & $5 / 6 / 2011$ & 28.02 & $24,000^{\mathrm{d}}$ \\
\hline 437 & 07047970 & $\begin{array}{l}\text { Mississippi River at Helena, } \\
\text { Arkansas }\end{array}$ & 941,800 & $04 / 1912$ & $54.30^{\mathrm{bb}, \mathrm{e}}$ & $2,040,000^{\mathrm{w}}$ & $1 / 132$ & $5 / 11 / 2011$ & 56.59 & $2,180,000^{\mathrm{d}}$ \\
\hline 438 & 07050700 & $\begin{array}{l}\text { James River near Springfield, } \\
\quad \text { Missouri }\end{array}$ & 246 & $07 / 1909$ & 22.00 & $62,000^{w, m}$ & $4 / 57$ & $4 / 26 / 2011$ & 18.85 & $30,600^{d}$ \\
\hline 439 & 07052250 & James River near Boaz, Missouri & 461 & $03 / 2008$ & 23.55 & $41,900^{\mathrm{d}}$ & $3 / 18$ & $4 / 25 / 2011$ & 20.82 & $30,500^{\mathrm{d}}$ \\
\hline 440 & 07052500 & $\begin{array}{l}\text { James River at Galena, Mis- } \\
\quad \text { souri }\end{array}$ & 987 & $03 / 2008$ & 35.96 & 85,100 & $3 / 90$ & $4 / 26 / 2011$ & $30.95^{h}$ & 64,000 \\
\hline 441 & 07057500 & $\begin{array}{l}\text { North Fork River near Tecum- } \\
\quad \text { seh, Missouri }\end{array}$ & 561 & $11 / 1985$ & 28.10 & 133,000 & $3 / 67$ & $4 / 26 / 2011$ & 24.58 & 81,000 \\
\hline 442 & 07058000 & $\begin{array}{l}\text { Bryant Creek near Tecumseh, } \\
\text { Missouri }\end{array}$ & 570 & $12 / 1982$ & 26.74 & 71,100 & $2 / 57$ & $4 / 26 / 2011$ & 23.01 & 47,600 \\
\hline 445 & 07062500 & Black River at Leeper, Missouri & 987 & 03/1904 & 22.30 & $125,000^{\mathrm{w}, \mathrm{m}}$ & $24 / 79$ & $4 / 24 / 2011$ & 12.98 & $11,700^{\mathrm{d}}$ \\
\hline 446 & 07063000 & $\begin{array}{l}\text { Black River at Poplar Bluff, } \\
\text { Missouri }\end{array}$ & 1,245 & 03/1904 & -- & $100,000^{\mathrm{j}, \mathrm{w}, \mathrm{m}}$ & $15 / 90$ & $4 / 26 / 2011$ & 21.41 & $24,400^{\mathrm{d}}$ \\
\hline 447 & 07064000 & $\begin{array}{l}\text { Black River near Corning, } \\
\text { Arkansas }\end{array}$ & 1,750 & $06 / 1945$ & 16.92 & 48,600 & $2 / 95$ & $4 / 28 / 2011$ & 18.12 & $40,700^{\mathrm{d}}$ \\
\hline 448 & 07068000 & $\begin{array}{l}\text { Current River at Doniphan, } \\
\text { Missouri }\end{array}$ & 2,038 & $03 / 1904$ & 24.90 & $130,000^{w, m}$ & $7 / 95$ & $4 / 26 / 2011$ & 23.76 & 90,100 \\
\hline 449 & 07069000 & $\begin{array}{l}\text { Black River at Pocahontas, } \\
\text { Arkansas }\end{array}$ & 4,840 & $04 / 1927$ & 25.90 & $80,000^{\mathrm{m}}$ & $1 / 76$ & $4 / 28 / 2011$ & 28.44 & $86,600^{\mathrm{d}}$ \\
\hline 450 & 07071500 & $\begin{array}{l}\text { Eleven Point River near Bard- } \\
\quad \text { ley, Missouri }\end{array}$ & 793 & $12 / 1982$ & 21.64 & 49,800 & $5 / 91$ & $4 / 26 / 2011$ & 17.83 & 33,400 \\
\hline 451 & 07072500 & $\begin{array}{l}\text { Black River at Black Rock, } \\
\text { Arkansas }\end{array}$ & 7,370 & $12 / 1982$ & $31.51^{j}$ & $190,000^{j}$ & $2 / 107$ & $4 / 26 / 2011$ & 30.45 & 172,000 \\
\hline
\end{tabular}




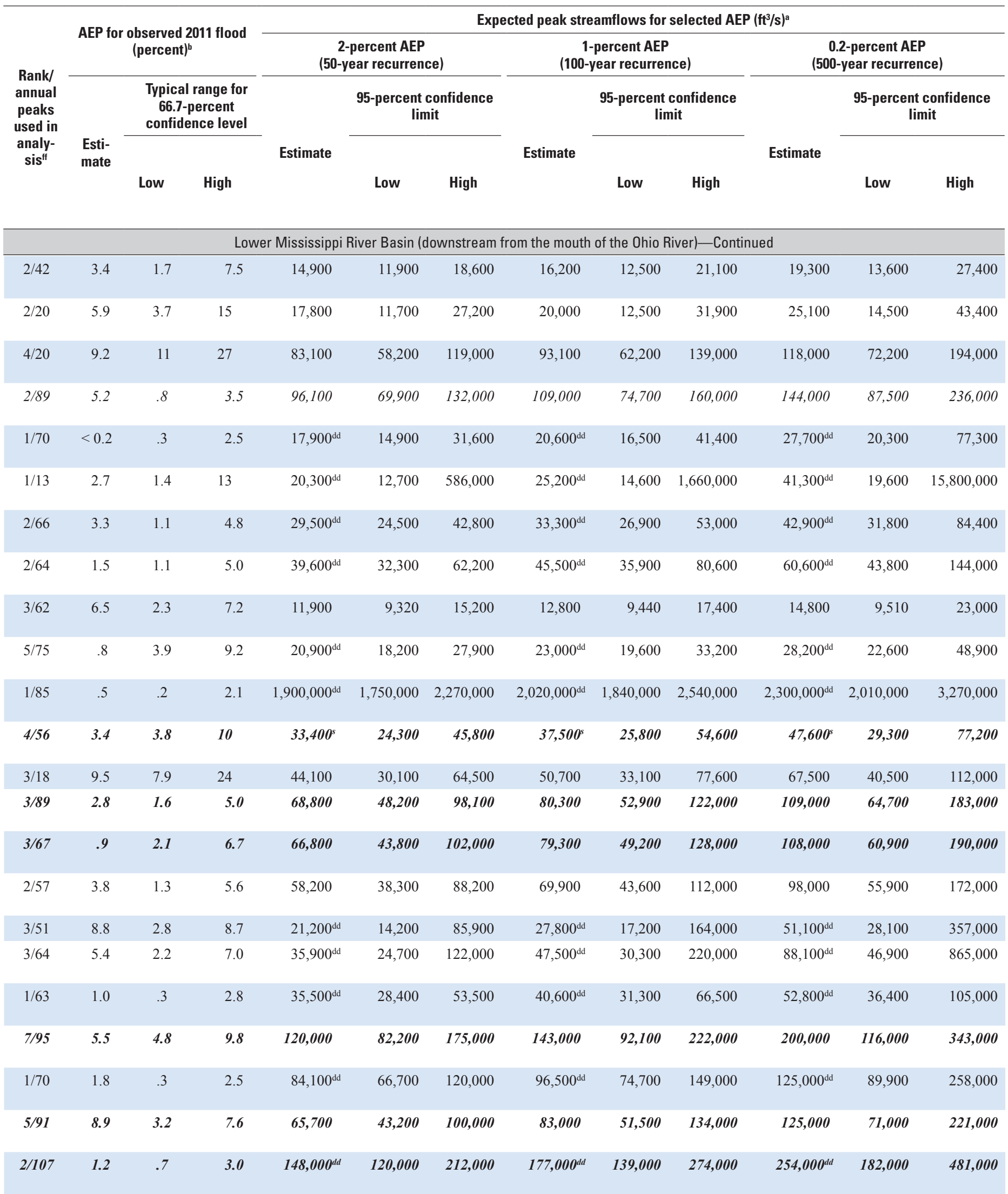




\section{Annual Exceedance Probabilities and Trends for the Central United States during the 2011 Floods}

Table 1. Peak streamflow data and results of flood-probability analyses for selected streamgages in the Central United States affected by 2011 flooding.-Continued

$\left[\mathrm{mi}^{2}\right.$, square miles; $\mathrm{ft}^{3} / \mathrm{s}$, cubic feet per second; AEP, annual exceedance probability; n/a, not applicable; --, data not available; <, less than; >, greater than] [Stations used in the trend analysis are italicized; Stations used in the trend analysis that overlap with trend analyses of Holmes and others (2010) are italicized and bold]

Flood data

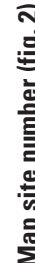

Contri-
buting
drainage
area
$\left(\mathrm{mi}^{2}\right)$

Previous maximum streamflow

Station name

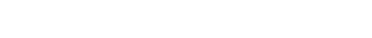

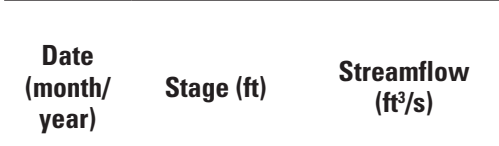

Floods of 2011

$\begin{array}{cccc}\begin{array}{c}\text { Rank }^{\mathrm{c}} / \\ \text { annual } \\ \text { peaks } \\ \text { in }\end{array} & \begin{array}{c}\text { Date of } \\ \text { peak } \\ \text { stream- }\end{array} & \begin{array}{c}\text { Peak stage } \\ \text { (ft) }\end{array} & \begin{array}{c}\text { Peak } \\ \text { streamflow } \\ \left(\mathrm{ft}^{3} / \mathrm{s}\right)\end{array} \\ \text { flow } & & & \end{array}$

\begin{tabular}{|c|c|c|c|c|c|c|c|c|c|c|}
\hline \multicolumn{11}{|c|}{ Lower Mississippi River Basin (downstream from the mouth of the Ohio River) - Continued } \\
\hline 452 & 07074420 & $\begin{array}{l}\text { Black River at Elgin Ferry, } \\
\text { Arkansas }\end{array}$ & 8,420 & $03 / 2008$ & $32.57^{\mathrm{h}}$ & $127,000^{j}$ & $1 / 24$ & $4 / 28 / 2011$ & $34.77^{\mathrm{e}}$ & 212,000 \\
\hline 453 & 07074500 & $\begin{array}{l}\text { White River at Newport, Ar- } \\
\text { kansas }\end{array}$ & 19,900 & $04 / 1945$ & $35.19^{\mathrm{j}, \mathrm{g}}$ & $343,000^{\mathrm{d}}$ & $6 / 126$ & $5 / 4 / 2011$ & 34.17 & $292,000^{\mathrm{d}}$ \\
\hline 454 & 07074850 & $\begin{array}{l}\text { White River near Augusta, } \\
\text { Arkansas }\end{array}$ & 20,500 & $03 / 2008$ & 38.41 & $252,000^{\mathrm{d}}$ & $1 / 74$ & $5 / 5 / 2011$ & 40.70 & $262,000^{\mathrm{d}}$ \\
\hline 456 & 07077380 & Cache River at Egypt, Arkansas & 701 & $01 / 1966$ & 21.88 & 8,940 & $2 / 62$ & $5 / 4 / 2011$ & 22.15 & 8,500 \\
\hline 457 & 07083710 & $\begin{array}{l}\text { Arkansas River below Empire } \\
\text { Gulch near Malta, Colorado }\end{array}$ & 238 & $06 / 2008$ & 5.41 & $1,530^{\mathrm{d}}$ & $1 / 12$ & $6 / 17 / 2011$ & 5.72 & $1,590^{\mathrm{d}}$ \\
\hline 458 & 07105800 & $\begin{array}{l}\text { Fountain Creek at Security, } \\
\text { Colorado }\end{array}$ & 500 & 07/1965 & 11.30 & 25,000 & $4 / 47$ & $9 / 14 / 2011$ & 9.48 & 13,200 \\
\hline 459 & 07105900 & $\begin{array}{l}\text { Jimmy Camp Creek at Fountain, } \\
\text { Colorado }\end{array}$ & 65.4 & $06 / 1965$ & -- & $124,000^{\mathrm{i}, \mathrm{m}}$ & $4 / 37$ & $9 / 15 / 2011$ & 10.46 & 4,120 \\
\hline 460 & 07106300 & $\begin{array}{l}\text { Fountain Creek near Pinon, } \\
\text { Colorado }\end{array}$ & 865 & 04/1999 & 9.80 & $19,100^{\mathrm{d}}$ & $2 / 39$ & $9 / 15 / 2011$ & 9.36 & $13,600^{\mathrm{d}}$ \\
\hline 469 & 07188653 & $\begin{array}{l}\text { Big Sugar Creek near Powell, } \\
\text { Missouri }\end{array}$ & 141 & $04 / 2008$ & 18.11 & 15,800 & $1 / 11$ & $4 / 25 / 2011$ & 21.99 & 24,000 \\
\hline 470 & 07188885 & $\begin{array}{l}\text { Indian Creek near Lanagan, } \\
\text { Missouri }\end{array}$ & 239 & $03 / 2008$ & 13.31 & 14,600 & $1 / 12$ & $5 / 24 / 2011$ & 14.07 & 16,100 \\
\hline 471 & 07189100 & $\begin{array}{l}\text { Buffalo Creek at Tiff City, Mis- } \\
\text { souri }\end{array}$ & 91.6 & $06 / 2007$ & 14.31 & 15,700 & $1 / 11$ & $5 / 24 / 2011$ & 15.28 & 26,300 \\
\hline 472 & 07189540 & $\begin{array}{l}\text { Cave Springs Branch near South- } \\
\text { west City, Missouri }\end{array}$ & 7.9 & $04 / 2008$ & 12.45 & 2,470 & $2 / 14$ & $5 / 23 / 2011$ & 12.30 & 2,340 \\
\hline 473 & 07189542 & $\begin{array}{l}\text { Honey Creek near Southwest } \\
\text { City, Missouri }\end{array}$ & 48.2 & $04 / 2008$ & 13.89 & 9,210 & $2 / 14$ & $5 / 24 / 2011$ & 13.27 & 7,020 \\
\hline 474 & 07191000 & $\begin{array}{l}\text { Big Cabin Creek near Big Cabin, } \\
\text { Oklahoma }\end{array}$ & 450 & 05/1943 & $34.96^{f}$ & 63,000 & $4 / 72$ & $5 / 24 / 2011$ & 48.74 & $45,900^{d}$ \\
\hline 476 & 07194800 & Illinois River at Savoy, Arkansas & 167 & $04 / 2004$ & 19.63 & 39,800 & $1 / 19$ & $4 / 25 / 2011$ & $24.66^{j}$ & 86,900 \\
\hline 477 & 07195000 & $\begin{array}{l}\text { Osage Creek near Elm Springs, } \\
\text { Arkansas }\end{array}$ & 130 & $05 / 1961$ & 16.66 & 22,500 & $1 / 46$ & $4 / 25 / 2011$ & 18.70 & 38,000 \\
\hline 478 & 07195400 & $\begin{array}{l}\text { Illinois River at Highway } 16 \text { near } \\
\text { Siloam Springs, Arkansas }\end{array}$ & 509 & $03 / 2008$ & 21.53 & 61,000 & $1 / 12$ & $4 / 26 / 2011$ & 26.23 & 87,100 \\
\hline 479 & 07195430 & $\begin{array}{l}\text { Illinois River south of Siloam } \\
\text { Springs, Arkansas }\end{array}$ & 575 & $04 / 2004$ & 20.54 & 52,000 & $1 / 16$ & $4 / 26 / 2011$ & 27.71 & 106,000 \\
\hline 480 & 07195500 & $\begin{array}{l}\text { Illinois River near Watts, Okla- } \\
\quad \text { homa }\end{array}$ & 635 & $07 / 1960$ & 25.96 & 68,000 & $1 / 56$ & $4 / 26 / 2011$ & 28.60 & $97,400^{d}$ \\
\hline 481 & 07196090 & $\begin{array}{l}\text { Illinois River at Chewey, Okla- } \\
\text { homa }\end{array}$ & 825 & $\mathrm{n} / \mathrm{a}$ & $\mathrm{n} / \mathrm{a}$ & $\mathrm{n} / \mathrm{a}$ & $1 / 1$ & $4 / 26 / 2011$ & 29.54 & 92,200 \\
\hline 482 & 07196500 & $\begin{array}{l}\text { Illinois River near Tahlequah, } \\
\text { Oklahoma }\end{array}$ & 959 & $05 / 1950$ & 27.94 & $150,000^{j}$ & $4 / 79$ & $4 / 26 / 2011$ & 25.97 & $85,400^{d}$ \\
\hline 483 & 07197000 & $\begin{array}{l}\text { Baron Fork at Eldon, Okla- } \\
\quad \text { homa }\end{array}$ & 307 & $06 / 2000$ & 26.77 & 54,700 & $1 / 65$ & $4 / 25 / 2011$ & 28.51 & $63,400^{d}$ \\
\hline 484 & 07197360 & $\begin{array}{l}\text { Caney Creek near Barber, } \\
\text { Oklahoma }\end{array}$ & 89.6 & $06 / 2000$ & $15.67^{\mathrm{f}, \mathrm{g}}$ & $9,720^{\mathrm{j}, 1}$ & $1 / 14$ & $4 / 25 / 2011$ & $31.42^{\mathrm{h}, \mathrm{e}}$ & $13,100^{\mathrm{j}}$ \\
\hline
\end{tabular}




\begin{tabular}{|c|c|c|c|c|c|c|c|c|c|c|c|c|}
\hline \multirow{4}{*}{$\begin{array}{c}\text { Rank/ } \\
\text { annual } \\
\text { peaks } \\
\text { used in } \\
\text { analy- } \\
\text { sis }^{f f}\end{array}$} & \multirow{2}{*}{\multicolumn{3}{|c|}{$\begin{array}{l}\text { AEP for observed } 2011 \text { flood } \\
\text { (percent) }^{\mathrm{b}}\end{array}$}} & \multicolumn{9}{|c|}{ Expected peak streamflows for selected AEP $\left(\mathrm{ft}^{3} / \mathrm{s}\right)^{\mathrm{a}}$} \\
\hline & & & & \multicolumn{3}{|c|}{$\begin{array}{c}\text { 2-percent AEP } \\
\text { (50-year recurrence) }\end{array}$} & \multicolumn{3}{|c|}{$\begin{array}{c}\text { 1-percent AEP } \\
\text { (100-year recurrence) }\end{array}$} & \multicolumn{3}{|c|}{$\begin{array}{c}\text { 0.2-percent AEP } \\
\text { (500-year recurrence) }\end{array}$} \\
\hline & \multirow{2}{*}{$\begin{array}{l}\text { Esti- } \\
\text { mate }\end{array}$} & \multicolumn{2}{|c|}{$\begin{array}{c}\text { Typical range for } \\
66.7-p e r c e n t \\
\text { confidence level }\end{array}$} & \multirow{2}{*}{ Estimate } & \multicolumn{2}{|c|}{$\begin{array}{l}\text { 95-percent confidence } \\
\text { limit }\end{array}$} & \multirow{2}{*}{ Estimate } & \multicolumn{2}{|c|}{$\begin{array}{l}\text { 95-percent confidence } \\
\text { limit }\end{array}$} & \multirow{2}{*}{ Estimate } & \multicolumn{2}{|c|}{$\begin{array}{c}\text { 95-percent confidence } \\
\text { limit }\end{array}$} \\
\hline & & Low & High & & Low & High & & Low & High & & Low & High \\
\hline \multicolumn{13}{|c|}{ Lower Mississippi River Basin (downstream from the mouth of the Ohio River)-Continued } \\
\hline$--/--$ & -- & -- & -- & -- & -- & -- & -- & -- & -- & -- & -- & -- \\
\hline $2 / 45$ & 2.7 & 1.6 & 7.0 & $317,000^{\mathrm{dd}}$ & 221,000 & $1,010,000$ & $399,000^{\mathrm{dd}}$ & 261,000 & $1,620,000$ & $649,000^{\mathrm{dd}}$ & 366,000 & $4,780,000$ \\
\hline --/-- & -- & -- & -- & -- & -- & -- & -- & -- & -- & -- & -- & -- \\
\hline $2 / 62$ & 2.0 & 1.2 & 5.1 & $8,490^{d d}$ & 7,450 & 11,000 & $9,290^{d d}$ & 7,990 & 12,800 & $11,200^{d d}$ & 9,130 & 18,000 \\
\hline $1 / 12$ & 8.5 & 1.5 & 14 & $2,010^{\mathrm{s}}$ & 1,470 & 5,480 & $2,210^{\mathrm{s}}$ & 1,520 & 7,070 & $2,640^{\mathrm{s}}$ & 1,520 & 12,200 \\
\hline $4 / 47$ & 6.9 & 4.6 & 12 & 18,400 & 12,700 & 26,700 & 21,800 & 14,100 & 33,500 & 30,500 & 17,300 & 53,800 \\
\hline $4 / 37$ & 6.1 & 5.8 & 15 & 7,070 & 3,500 & 14,300 & 9,530 & 4,340 & 20,900 & 16,700 & 6,400 & 43,800 \\
\hline $2 / 39$ & 3.7 & 1.9 & 8.1 & $17,500^{\mathrm{s}}$ & 11,300 & 83,800 & $22,900^{s}$ & 13,700 & 157,000 & $41,100^{\mathrm{s}}$ & 20,100 & 693,000 \\
\hline $1 / 11$ & 3.6 & 1.6 & 15 & 27,800 & 16,700 & 46,300 & 32,700 & 19,000 & 56,300 & 44,700 & 24,500 & 81,300 \\
\hline $1 / 12$ & 9.1 & 1.5 & 14 & 22,700 & 16,300 & 31,600 & 26,200 & 18,100 & 37,900 & 35,500 & 22,600 & 55,700 \\
\hline $1 / 11$ & .6 & 1.6 & 15 & 19,300 & 11,500 & 32,500 & 22,600 & 13,000 & 39,200 & 30,100 & 16,400 & 55,100 \\
\hline $2 / 14$ & 9.5 & 5.3 & 21 & 4,370 & 2,710 & 7,040 & 5,430 & 3,220 & 9,140 & 8,170 & 4,280 & 15,600 \\
\hline $2 / 14$ & 7.0 & 5.3 & 21 & 18,000 & 10,300 & 31,600 & 22,200 & 12,200 & 40,500 & 33,600 & 16,100 & 69,800 \\
\hline $1 / 25$ & 3.2 & .7 & 6.9 & $49,200^{s}$ & 34,900 & 82,300 & $54,500^{s}$ & 34,900 & 97,000 & $65,700^{5}$ & 34,900 & 141,000 \\
\hline $1 / 19$ & 1.2 & 1.0 & 9.0 & $72,700^{\mathrm{dd}}$ & 41,500 & 182,000 & $90,500^{\mathrm{dd}}$ & 49,100 & 276,000 & $139,000^{\mathrm{dd}}$ & 65,000 & 692,000 \\
\hline $1 / 46$ & .9 & .4 & 3.8 & $28,800^{\mathrm{dd}}$ & 19,200 & 56,300 & $36,600^{\mathrm{dd}}$ & 22,800 & 81,400 & $58,400^{\mathrm{dd}}$ & 30,300 & 176,000 \\
\hline $1 / 12$ & 3.6 & 1.5 & 14 & $107,000^{\mathrm{dd}}$ & 53,900 & 286,000 & $134,000^{\mathrm{dd}}$ & 64,300 & 441,000 & $206,000^{\mathrm{dd}}$ & 85,200 & $1,170,000$ \\
\hline $1 / 16$ & 1.1 & 1.1 & 11 & $92,500^{\mathrm{dd}}$ & 59,600 & 296,000 & $108,000^{\mathrm{dd}}$ & 66,900 & 448,000 & $148,000^{\mathrm{dd}}$ & 81,000 & 941,000 \\
\hline $1 / 31$ & 1.0 & .6 & 5.6 & $79,500^{s}$ & 54,700 & 259,000 & $96,400^{s}$ & 62,800 & 388,000 & $143,000^{5}$ & 80,900 & 980,000 \\
\hline$--/--$ & -- & -- & -- & $90,200^{r}$ & -- & -- & $104,000^{r}$ & -- & -- & $150,000^{\mathrm{r}}$ & -- & -- \\
\hline $1 / 25$ & 2.4 & .7 & 6.9 & $89,100^{s}$ & 58,500 & 433,000 & $110,000^{5}$ & 67,800 & 721,000 & $170,000^{5}$ & 88,700 & $2,370,000$ \\
\hline $1 / 65$ & 1.9 & .3 & 2.7 & $62,500^{s}$ & 48,200 & 95,400 & $72,600^{s}$ & 53,800 & 121,000 & $96,200^{s}$ & 63,700 & 201,000 \\
\hline $1 / 14$ & 6.7 & 1.3 & 12 & 18,300 & 12,500 & 26,900 & 21,700 & 14,200 & 33,200 & 30,200 & 17,700 & 51,700 \\
\hline
\end{tabular}




\section{Annual Exceedance Probabilities and Trends for the Central United States during the 2011 Floods}

Table 1. Peak streamflow data and results of flood-probability analyses for selected streamgages in the Central United States affected by 2011 flooding.-Continued

$\left[\mathrm{mi}^{2}\right.$, square miles; $\mathrm{ft}^{3} / \mathrm{s}$, cubic feet per second; AEP, annual exceedance probability; n/a, not applicable; --, data not available; <, less than; $>$, greater than] [Stations used in the trend analysis are italicized; Stations used in the trend analysis that overlap with trend analyses of Holmes and others (2010) are italicized and bold]

\section{Flood data}

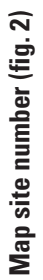

Contri-

buting

Site

number

Station name

drainage

area

$\left(\mathrm{mi}^{2}\right)$
Previous maximum streamflow

Floods of 2011

Date
$\begin{gathered}\text { (month/ } \\ \text { year) }\end{gathered}$ Stage $(\mathrm{ft}) \quad \begin{gathered}\text { Streamflow } \\ \left(\mathrm{ft}^{3} / \mathbf{s}\right)\end{gathered}$

\begin{tabular}{|c|c|c|c|}
\hline $\begin{array}{l}\text { Rank }^{c} / \\
\text { annual } \\
\text { peaks } \\
\text { in }\end{array}$ & $\begin{array}{l}\text { Date of } \\
\text { peak } \\
\text { stream- } \\
\text { flow }\end{array}$ & $\begin{array}{c}\text { Peak stage } \\
\text { (ft) }\end{array}$ & $\begin{array}{c}\text { Peak } \\
\text { streamflow } \\
\left(\mathrm{ft}^{3} / \mathbf{s}\right)\end{array}$ \\
\hline
\end{tabular}

Lower Mississippi River Basin (downstream from the mouth of the Ohio River)-Continued

\begin{tabular}{|c|c|c|c|c|c|c|c|c|c|c|}
\hline 485 & 07198000 & $\begin{array}{l}\text { Illinois River near Gore, Okla- } \\
\quad \text { homa }\end{array}$ & 1,626 & $05 / 1950$ & $30.20^{f}$ & 180,000 & $14 / 73$ & $5 / 24 / 2011$ & 17.57 & $15,900^{d}$ \\
\hline 487 & 07249985 & Lee Creek near Short, Oklahoma & 420 & $04 / 2004$ & $27.77^{\mathrm{g}}$ & 82,400 & $3 / 66$ & $4 / 26 / 2011$ & 26.08 & 70,100 \\
\hline 488 & 07250965 & Frog Bayou at Winfrey, Arkansas & 54.2 & $04 / 2004$ & 11.58 & 12,900 & $1 / 9$ & $4 / 25 / 2011$ & 13.88 & 21,800 \\
\hline 489 & 07265450 & $\begin{array}{l}\text { Mississippi River at Arkansas } \\
\text { City, Arkansas }\end{array}$ & $1,130,600$ & $05 / 1927$ & $59.20^{\mathrm{e}}$ & $2,500,000^{\mathrm{d}, \mathrm{w}, \mathrm{ee}}$ & $2 / 88$ & $5 / 17 / 2011$ & 53.14 & $2,400,000^{\mathrm{j}, \mathrm{d}}$ \\
\hline 490 & 07289000 & $\begin{array}{l}\text { Mississippi River at Vicksburg, } \\
\text { Mississippi }\end{array}$ & $1,140,500$ & $05 / 1927$ & $56.20^{\mathrm{e}}$ & $2,278,000^{\mathrm{j}}$ & $1 / 91$ & $5 / 17 / 2011$ & 57.17 & $2,310,000^{\mathrm{d}, \mathrm{l}}$ \\
\hline 492 & 07374000 & $\begin{array}{l}\text { Mississippi River at Baton } \\
\text { Rouge, Louisiana }\end{array}$ & $1,125,810$ & $04 / 1945$ & 45.18 & $1,473,000$ & $2 / 36$ & $5 / 18 / 2011$ & 45.48 & $1,440,000^{\mathrm{d}}$ \\
\hline 493 & 07374525 & $\begin{array}{l}\text { Mississippi River at Belle Chase, } \\
\text { Louisiana }\end{array}$ & $1,110,000$ & $02 / 2010$ & $15.84^{\mathrm{g}}$ & $1,210,000$ & $1 / 3$ & $5 / 17 / 2011$ & $18.28^{\mathrm{e}}$ & $1,320,000$ \\
\hline 494 & 07381482 & $\begin{array}{l}\text { Old River Outflow Channel } \\
\text { below Hydropower Channel, } \\
\text { Louisiana }\end{array}$ & $--^{k}$ & $04 / 2010$ & $35.08^{\mathrm{g}}$ & 423,000 & $1 / 3$ & $5 / 20 / 2011$ & $49.82^{\mathrm{e}}$ & 730,000 \\
\hline 495 & 07381490 & $\begin{array}{l}\text { Atchafalaya River at Simmes- } \\
\text { port, Louisiana }\end{array}$ & $--^{k}$ & $05 / 1973$ & 53.40 & 781,000 & $3 / 9$ & $5 / 24 / 2011$ & 45.06 & 697,000 \\
\hline 496 & 07381590 & $\begin{array}{l}\text { Wax Lake Outlet at Calumet, } \\
\text { Louisiana }\end{array}$ & $--^{k}$ & $04 / 1997$ & -- & $258,000^{1}$ & $1 / 67$ & $5 / 27 / 2011$ & 10.81 & 323,000 \\
\hline 497 & 07381600 & $\begin{array}{l}\text { Lower Atchafalaya River at } \\
\text { Morgan City, Louisiana }\end{array}$ & $--^{k}$ & $04 / 2008$ & $7.81^{\mathrm{g}}$ & 366,000 & $1 / 34$ & $5 / 29 / 2011$ & $10.33^{\mathrm{e}}$ & 512,000 \\
\hline
\end{tabular}

${ }^{a}$ Unless otherwise noted, expected peak streamflows are based on Water Resources Council Bulletin 17B weighting by variance method.

${ }^{b}$ Where frequency analysis was performed by USGS according to Bulletin 17B methods, the AEP estimate listed here is interpolated from expected peak streamflows (in columns at right). $66.7 \%$ confidence limits are computed using non-parametric methods based on the rank and annual peaks used in the USGS frequency analysis. No estimated AEP or confidence limits are computed here for the 2011 peak streamflow if the expected peak streamflows were furnished by other agencies, computed through regional regression equations, or unavailable.

${ }^{\mathrm{c}}$ Rank of the maximum instantaneous peak streamflow measured during 2011 event compared to all systematic and historic annual peaks. A rank of 1 indicates that the 2011 peak streamflow was higher than all other recorded annual peaks.

${ }^{\mathrm{S} S t r e a m f l o w}$ was affected by regulation or diversion.

eStage corresponds to maximum stage for the flood, not the stage when the peak streamflow occurred.

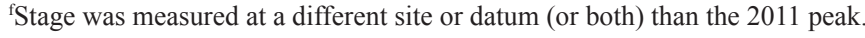

${ }^{\mathrm{g}}$ Stage is the stage of peak streamflow, not the maximum historic stage.

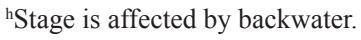

${ }^{\mathrm{i}} \mathrm{A}$ previous historic peak stage and unknown streamflow exists that likely exceeds the previous max streamflow.

${ }^{\mathrm{j} E s t i m a t e d}$

${ }^{k}$ Major river diversions upstream cause these drainage areas to be indeterminate.

'Streamflow is a maximum daily average. Peak streamflow may be higher.

${ }^{\mathrm{m}}$ Streamflow is an historic peak.

${ }^{\mathrm{n}}$ Streamflow is unknown but greater than the reported value.

${ }^{\circ} \mathrm{All}$ or part of the record is affected by urbanization, mining, agricultural changes, channelization, or other

PStreamflow was affected by dam failure.

${ }^{9}$ Source: U.S. Army Corps of Engineers (2004).

${ }^{\mathrm{r}}$ Expected peak streamflow based on regional regression equation estimate only. 


\begin{tabular}{|c|c|c|c|c|c|c|c|c|c|c|c|c|}
\hline \multirow{4}{*}{$\begin{array}{c}\text { Rank/ } \\
\text { annual } \\
\text { peaks } \\
\text { used in } \\
\text { analy- } \\
\text { sis }^{f f}\end{array}$} & \multirow{2}{*}{\multicolumn{3}{|c|}{$\begin{array}{l}\text { AEP for observed } 2011 \text { flood } \\
\text { (percent) }^{\mathrm{b}}\end{array}$}} & \multicolumn{9}{|c|}{ Expected peak streamflows for selected AEP $\left(\mathrm{ft}^{3} / \mathrm{s}\right)^{\mathrm{a}}$} \\
\hline & & & & \multicolumn{3}{|c|}{$\begin{array}{c}\text { 2-percent AEP } \\
\text { (50-year recurrence) }\end{array}$} & \multicolumn{3}{|c|}{$\begin{array}{c}\text { 1-percent AEP } \\
\text { (100-year recurrence) }\end{array}$} & \multicolumn{3}{|c|}{$\begin{array}{c}\text { 0.2-percent AEP } \\
\text { (500-year recurrence) }\end{array}$} \\
\hline & \multirow{2}{*}{$\begin{array}{l}\text { Esti- } \\
\text { mate }\end{array}$} & \multicolumn{2}{|c|}{$\begin{array}{l}\text { Typical range for } \\
66.7-p e r c e n t \\
\text { confidence level }\end{array}$} & \multirow{2}{*}{ Estimate } & \multicolumn{2}{|c|}{$\begin{array}{l}\text { 95-percent confidence } \\
\text { limit }\end{array}$} & \multirow{2}{*}{ Estimate } & \multicolumn{2}{|c|}{$\begin{array}{l}\text { 95-percent confidence } \\
\text { limit }\end{array}$} & \multirow{2}{*}{ Estimate } & \multicolumn{2}{|c|}{$\begin{array}{c}\text { 95-percent confidence } \\
\text { limit }\end{array}$} \\
\hline & & Low & High & & Low & High & & Low & High & & Low & High \\
\hline \multicolumn{13}{|c|}{ Lower Mississippi River Basin (downstream from the mouth of the Ohio River)-Continued } \\
\hline $3 / 61$ & 7.2 & 2.3 & 7.3 & $19,900^{s}$ & 16,800 & 38,100 & $22,600^{s}$ & 18,400 & 52,200 & $29,600^{5}$ & 22,000 & 110,000 \\
\hline $3 / 66$ & 2.1 & 2.1 & 6.8 & $70,900^{\mathrm{dd}}$ & 56,400 & 95,000 & $86,100^{\text {dd }}$ & 67,100 & 119,000 & $127,000^{\mathrm{dd}}$ & 95,000 & 186,000 \\
\hline $1 / 10$ & 2.0 & 1.8 & 16 & $21,700^{\mathrm{dd}}$ & 16,000 & 40,100 & $24,600^{\mathrm{dd}}$ & 17,600 & 48,700 & $31,700^{\mathrm{dd}}$ & 21,400 & 73,100 \\
\hline --/-- & -- & -- & -- & -- & -- & -- & -- & -- & -- & -- & -- & -- \\
\hline $1 / 87$ & .8 & .2 & 2.0 & $2,120,000^{\mathrm{s}}$ & $1,980,000$ & $2,520,000$ & $2,240,000^{s}$ & $2,070,000$ & $2,840,000$ & $2,490,000^{\mathrm{s}}$ & $2,250,000$ & $3,500,000$ \\
\hline --/-- & -- & -- & -- & -- & -- & -- & -- & -- & -- & -- & -- & -- \\
\hline --/-- & -- & -- & -- & -- & -- & -- & -- & -- & -- & -- & -- & -- \\
\hline --/-- & -- & -- & -- & -- & -- & -- & -- & -- & -- & -- & -- & -- \\
\hline --/-- & -- & -- & -- & -- & -- & -- & -- & -- & -- & -- & -- & -- \\
\hline --/-- & -- & -- & -- & -- & -- & -- & -- & -- & -- & -- & -- & -- \\
\hline --/-- & -- & -- & -- & -- & -- & -- & -- & -- & -- & -- & -- & -- \\
\hline
\end{tabular}

${ }^{\mathrm{s}}$ Expected peak streamflow based on Water Resources Council Bulletin 17B at-site estimate only.

'No USGS streamgage is currently in operation at this site. Streamflow is provided by AmerenUE and computed from operation of turbines in power plant and spillway gates at dam.

uSource: U.S. Army Corps of Engineers (2010), Des Moines River Regulated Flow Frequency Study, Rock Island, Illinois., 82p.

${ }^{\vee}$ Expected peak streamflows determined using drainage area ratio adjustment method as described in WRIR 03-4308 by Parrett and Johnson, 2003 , p. 34.

${ }^{w}$ Month or day of occurrence is unknown or not exact.

${ }^{x}$ Source: Sando and others (2008), Peak-flow frequency estimates based on data through water year 2001 for selected streamflow-gaging stations in South Dakota: U.S. Geological Survey Scientific Investigations Report 2008-5104, 367 p

${ }^{y}$ No stage data available. 2011 Streamflow measured directly at site where stage gage is not established or has been discontinued.

${ }^{2}$ No flood frequency analysis available. Peak records furnished by Wyoming State Engineer's Office.

aaThis value is for Hickman, Kentucky which is 29.6 river miles downstream from Wickliffe, Kentucky and includes the flow through the New Madrid Floodway (Mississippi River Commission, 1955). The 1912 and 1913 maximum streamflows were 2,015,000 ft 3 /s (Mississippi River Commission, 1955).

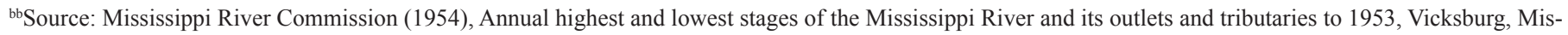
sissippi, 253 p.

${ }^{\mathrm{cc} S o u r c e: ~ M i s s i s s i p p i ~ R i v e r ~ C o m m i s s i o n ~(1955) . ~}$

dd Source: Westerman and others (2012).

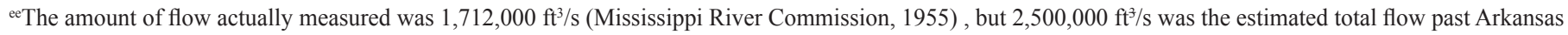
City factoring in that flow which was bypassing the main channel area through the ruptured levees (written communication, 2012, Charles Shadie, Chief, Water Control, Mississippi Valley Division, U.S. Army Corps of Engineers).

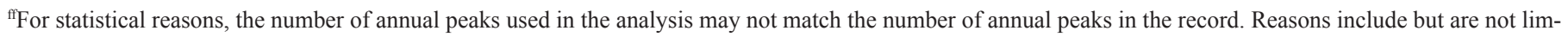
ited to period extensions from nearby gages and mixed periods of regulation and non-regulation.

${ }^{g g}$ Source: U.S. Army Corps of Engineers (2001). 
Table 2. Annual runoff data and results of flood-probability analyses for selected streamgages in the Central United States affected by 2011 flooding.

[Stations used in the trend analysis are italicized. $\mathrm{mi}^{2}$, square miles; AEP, annual exceedance probability; <, less than; >, greater than]

\begin{tabular}{|c|c|c|c|c|c|c|c|c|c|c|c|}
\hline \multirow{2}{*}{ 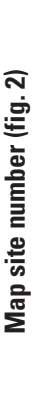 } & \multirow[b]{2}{*}{$\begin{array}{l}\text { Station } \\
\text { number }\end{array}$} & \multirow[b]{2}{*}{ Station name } & \multirow{2}{*}{ 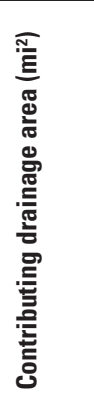 } & \multicolumn{2}{|c|}{ Water year 2011} & \multicolumn{4}{|c|}{ Period of record } & \multicolumn{2}{|c|}{$\begin{array}{l}\text { Previous maximum } \\
\text { annual runoff based } \\
\text { on data through } \\
\text { water year } 2010^{\mathrm{a}}\end{array}$} \\
\hline & & & & 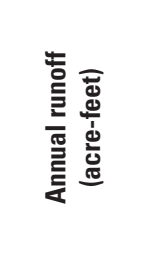 & 兰 & 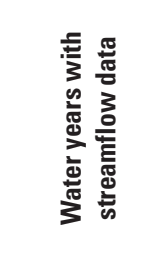 & 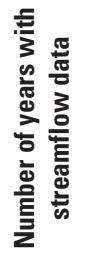 & 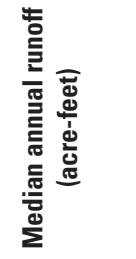 & 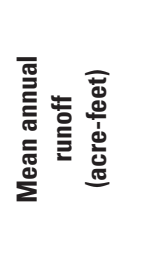 & 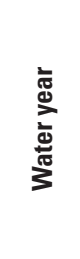 & 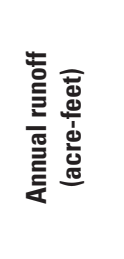 \\
\hline \multicolumn{12}{|c|}{ Red River of the North Basin } \\
\hline 2 & 05046000 & $\begin{array}{l}\text { Otter Tail River below Orwell } \\
\text { Dam near Fergus Falls, } \\
\text { Minnesota }\end{array}$ & 1,740 & $1,050,000$ & 1 & $1931-2011$ & 81 & 255,600 & 295,700 & 2009 & 679,100 \\
\hline 4 & 05050000 & $\begin{array}{l}\text { Bois De Sioux River near } \\
\quad \text { White Rock, South Dakota }\end{array}$ & 1,160 & 607,400 & 1 & $1942-2011$ & 70 & 59,000 & 100,300 & 1997 & 388,100 \\
\hline 5 & 05051500 & $\begin{array}{l}\text { Red River of the North at Wah- } \\
\text { peton, North Dakota }\end{array}$ & 4,010 & $2,005,000$ & 1 & 1944-2011 & 68 & 453,600 & 531,000 & 2009 & $1,332,000$ \\
\hline 9 & 05053000 & $\begin{array}{l}\text { Wild Rice River near Aber- } \\
\text { crombie, North Dakota }\end{array}$ & 1,490 & 854,300 & 1 & $1933-2011$ & 79 & 41,270 & 106,400 & 2009 & 697,200 \\
\hline 10 & 05054000 & $\begin{array}{l}\text { Red River of the North at } \\
\text { Fargo, North Dakota }\end{array}$ & 6,800 & $3,214,000$ & 1 & $1902-2011$ & 110 & 417,400 & 580,800 & 2009 & $2,527,000$ \\
\hline 12 & 05056000 & $\begin{array}{l}\text { Sheyenne River near Warwick, } \\
\text { North Dakota }\end{array}$ & 760 & 345,300 & 1 & $1950-2011$ & 62 & 44,890 & 62,890 & 2009 & 272,900 \\
\hline 22 & 05059500 & $\begin{array}{l}\text { Sheyenne River at West Fargo, } \\
\text { North Dakota }\end{array}$ & 3,090 & $1,267,000$ & 1 & $\begin{array}{c}1904-05 \\
1930- \\
2011\end{array}$ & 84 & 133,900 & 199,300 & 2010 & 752,900 \\
\hline 27 & 05062000 & $\begin{array}{l}\text { Buffalo River near Dilworth, } \\
\text { Minnesota }\end{array}$ & 975 & 460,400 & 2 & $1932-2011$ & 80 & 108,200 & 131,900 & 2009 & 526,300 \\
\hline 28 & 05064000 & $\begin{array}{l}\text { Wild Rice at Hendrum, Min- } \\
\text { nesota }\end{array}$ & 1,560 & 760,200 & 1 & $\begin{array}{c}1945-84 \\
1986- \\
2011\end{array}$ & 66 & 228,800 & 268,500 & 2009 & 738,500 \\
\hline 31 & 05066500 & $\begin{array}{l}\text { Goose River at Hillsboro, } \\
\text { North Dakota }\end{array}$ & 1,093 & 485,800 & 1 & $\begin{array}{l}1932,1935- \\
2011\end{array}$ & 78 & 44,890 & 83,450 & 2009 & 298,300 \\
\hline 32 & 05069000 & $\begin{array}{l}\text { Sand Hill River at Climax, } \\
\text { Minnesota }\end{array}$ & 420 & 186,800 & 1 & $\begin{array}{c}1947-84 \\
1986- \\
2011\end{array}$ & 64 & 60,810 & 67,650 & 1999 & 180,300 \\
\hline 35 & 05079000 & $\begin{array}{l}\text { Red Lake River at Crookston, } \\
\quad \text { Minnesota }\end{array}$ & 5,270 & $1,911,000$ & 4 & $1902-2011$ & 110 & 810,900 & 893,000 & 1950 & $2,266,000$ \\
\hline 36 & 05082500 & $\begin{array}{l}\text { Red River of the North at } \\
\text { Grand Forks, North Dakota }\end{array}$ & 26,300 & $10,350,000$ & 1 & $1905-2011$ & 107 & $1,911,000$ & $2,405,000$ & 2009 & $7,964,000$ \\
\hline 38 & 05085000 & $\begin{array}{l}\text { Forest River at Minto, North } \\
\text { Dakota }\end{array}$ & 620 & 96,290 & 7 & 1945-2011 & 67 & 35,470 & 43,230 & 2009 & 198,400 \\
\hline 39 & 05087500 & $\begin{array}{l}\text { Middle River at Argyle, Min- } \\
\text { nesota }\end{array}$ & 255 & 113,700 & 3 & $\begin{array}{c}1951-81 \\
1983- \\
2011\end{array}$ & 60 & 34,030 & 41,270 & 1999 & 143,300 \\
\hline 40 & 05090000 & $\begin{array}{l}\text { Park River at Grafton, North } \\
\text { Dakota }\end{array}$ & 695 & 144,800 & 4 & 1932-2011 & 80 & 40,900 & 51,400 & 2009 & 259,900 \\
\hline 41 & 05092000 & $\begin{array}{l}\text { Red River of the North at } \\
\text { Drayton, North Dakota }\end{array}$ & 34,800 & $11,150,000$ & 1 & 1950-2011 & 62 & $3,334,000$ & $3,570,000$ & 2009 & $9,846,000$ \\
\hline 42 & 05094000 & $\begin{array}{l}\text { South Branch Two Rivers at } \\
\text { Lake Bronson, Minnesota }\end{array}$ & 422 & 231,700 & 2 & $\begin{array}{l}\text { 1929-36, } \\
1942-43, \\
1946-47, \\
1954-81, \\
1986- \\
2011\end{array}$ & 66 & 54,300 & 83,420 & 2009 & 237,500 \\
\hline
\end{tabular}


Estimated

Expected annual runoff in acre-feet for selected annual exceedance probabilities (AEP) based on Water Resources Council Bulletin 17B methods

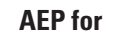

observed

2011

annual

runoff

(percent)

\begin{tabular}{c}
\hline $\begin{array}{c}\text { 2-percent AEP } \\
(50-y e a r \text { recurrence })\end{array}$ \\
$\frac{95-\text { percent confidence limit }}{}$
\end{tabular}

\begin{tabular}{c}
$\begin{array}{c}\text { 1-percent AEP } \\
(100-y e a r \text { recurrence })\end{array}$ \\
\hline 95-percent confidence limit \\
\hline
\end{tabular}

$(500$

Estimate
Estimate

High

Low

High

0.2-percent AEP

(500-year recurrence)

95-percent confidence limit

Low
High

\begin{tabular}{|c|c|c|c|c|c|c|c|c|c|}
\hline \multicolumn{10}{|c|}{ Red River of the North Basin } \\
\hline$<0.2$ & 720,400 & 270,700 & 956,400 & 758,600 & 240,000 & $1,095,000$ & 814,200 & 311,000 & $1,485,000$ \\
\hline 2 to 4 & 676,500 & 222,700 & $1,320,000$ & 845,200 & 187,300 & $1,882,000$ & $1,229,000$ & 156,100 & $4,004,000$ \\
\hline$<0.2$ & $1,412,000$ & $1,093,000$ & $1,933,000$ & $1,583,000$ & $1,132,000$ & $2,283,000$ & $1,954,000$ & $1,152,000$ & $3,241,000$ \\
\hline 1 to 2 & 715,200 & 422,400 & $1,533,000$ & 970,600 & 504,400 & $2,374,000$ & $1,729,000$ & 639,800 & $5,913,000$ \\
\hline 0.2 to 1 & $2,147,000$ & $1,545,000$ & $3,014,000$ & $2,514,000$ & $1,626,000$ & $3,755,000$ & $3,335,000$ & $1,701,000$ & $5,890,000$ \\
\hline 0.2 to 1 & 266,500 & 183,300 & 508,100 & 331,000 & 211,100 & 709,700 & 503,700 & 260,900 & $1,436,000$ \\
\hline 0.2 to 1 & 850,900 & 606,900 & $1,658,000$ & $1,098,000$ & 740,400 & $2,532,000$ & $1,853,000$ & $1,081,000$ & $6,450,000$ \\
\hline 2 to 4 & 463,000 & 351,000 & 731,600 & 555,600 & 399,100 & 958,800 & 792,900 & 491,700 & $1,692,000$ \\
\hline 2 to 4 & 800,200 & 613,500 & $1,185,000$ & 921,000 & 660,700 & $1,457,000$ & $1,201,000$ & 721,800 & $2,247,000$ \\
\hline 1 to 2 & 468,100 & 302,400 & 902,800 & 607,400 & 354,900 & $1,315,000$ & 997,700 & 445,000 & $2,883,000$ \\
\hline 2 to 4 & 212,700 & 162,500 & 351,500 & 251,600 & 183,300 & 460,700 & 350,600 & 224,100 & 818,400 \\
\hline
\end{tabular}

\begin{tabular}{|c|c|c|c|c|c|c|c|c|c|}
\hline 4 to 10 & $2,443,000$ & $1,591,000$ & $3,095,000$ & $2,672,000$ & $1,535,000$ & $3,571,000$ & $3,092,000$ & $1,509,000$ & $4,782,000$ \\
\hline 0.2 to 1 & $8,087,000$ & $6,298,000$ & $11,220,000$ & $9,462,000$ & $6,911,000$ & $13,920,000$ & $12,700,000$ & $7,857,000$ & $21,700,000$ \\
\hline 4 to 10 & 176,700 & 125,100 & 320,200 & 218,000 & 144,100 & 441,700 & 328,000 & 179,900 & 869,200 \\
\hline 4 to 10 & 151,400 & 66,220 & 232,600 & 172,500 & 56,350 & 288,800 & 214,500 & 49,860 & 460,900 \\
\hline
\end{tabular}

\begin{tabular}{|c|c|c|c|c|c|c|c|c|c|}
\hline 4 to 10 & 248,800 & 134,100 & 405,600 & 298,800 & 128,600 & 533,900 & 410,000 & 117,500 & 948,000 \\
\hline 1 to 2 & $10,360,000$ & $7,808,000$ & $15,040,000$ & $11,790,000$ & $8,173,000$ & $18,170,000$ & $14,970,000$ & $8,414,000$ & $27,110,000$ \\
\hline
\end{tabular}

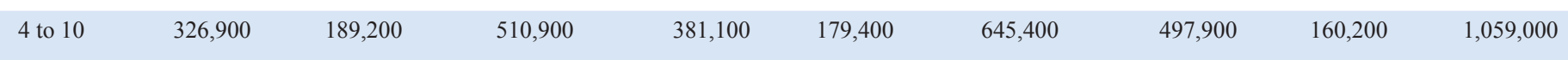


Table 2. Annual runoff data and results of flood-probability analyses for selected streamgages in the Central United States affected by 2011 flooding.-Continued

[Stations used in the trend analysis are italicized. $\mathrm{mi}^{2}$, square miles; AEP, annual exceedance probability; <, less than; >, greater than]

\begin{tabular}{|c|c|c|c|c|c|c|c|c|c|c|c|}
\hline \multirow{2}{*}{ 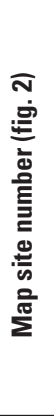 } & \multirow[b]{2}{*}{$\begin{array}{l}\text { Station } \\
\text { number }\end{array}$} & \multirow[b]{2}{*}{ Station name } & \multirow{2}{*}{ 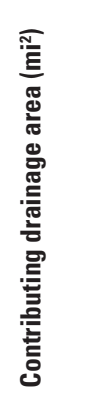 } & \multicolumn{2}{|c|}{ Water year 2011} & \multicolumn{4}{|c|}{ Period of record } & \multicolumn{2}{|c|}{$\begin{array}{l}\text { Previous maximum } \\
\text { annual runoff based } \\
\text { on data through } \\
\text { water year } 2010^{\text {a }}\end{array}$} \\
\hline & & & & 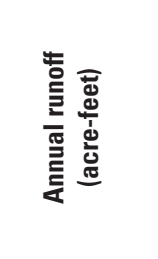 & 兰 & 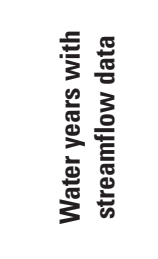 & 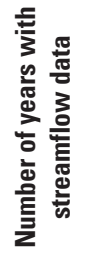 & 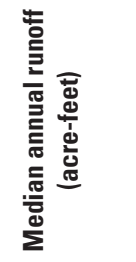 & 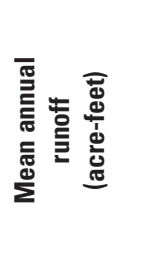 & 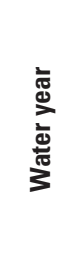 & 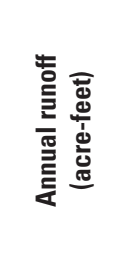 \\
\hline \multicolumn{12}{|c|}{ Red River of the North Basin-Continued } \\
\hline 44 & 05100000 & $\begin{array}{l}\text { Pembina River at Neche, North } \\
\quad \text { Dakota }\end{array}$ & 3,410 & $1,050,000$ & 1 & $\begin{array}{l}\text { 1904-08, } \\
\quad 1910-15 \\
1920- \\
2011\end{array}$ & 103 & 115,100 & 189,700 & 1995 & 810,900 \\
\hline 45 & 05102500 & $\begin{array}{l}\text { Red River at Emerson, Mani- } \\
\quad \text { toba }\end{array}$ & 40,200 & $12,810,000$ & 1 & $1913-2011$ & 99 & $2,324,000$ & $3,184,000$ & 2009 & $10,930,000$ \\
\hline \multicolumn{12}{|c|}{ Souris River Basin } \\
\hline 46 & 05113600 & $\begin{array}{l}\text { Long Creek near Noonan, } \\
\text { North Dakota }\end{array}$ & 630 & 390,200 & 1 & $1960-2011$ & 52 & 15,200 & 35,350 & 1976 & 145,500 \\
\hline 47 & 05114000 & $\begin{array}{l}\text { Souris River near Sherwood, } \\
\quad \text { North Dakota }\end{array}$ & 3,040 & $1,643,000$ & 1 & $1931-2011$ & 81 & 45,610 & 106,900 & 1976 & 637,100 \\
\hline 49 & 05116500 & $\begin{array}{l}\text { Des Lacs River at Foxholm, } \\
\text { North Dakota }\end{array}$ & 539 & 219,400 & 1 & $\begin{array}{c}1905-06 \\
1946- \\
2011\end{array}$ & 68 & 10,860 & 22,260 & 1976 & 107,100 \\
\hline 50 & 05117500 & $\begin{array}{l}\text { Souris River above Minot, } \\
\text { North Dakota }\end{array}$ & 3,900 & $1,969,000$ & 1 & 1904-2011 & 108 & 60,450 & 127,700 & 1976 & 803,600 \\
\hline 52 & 05120500 & $\begin{array}{l}\text { Wintering River near Karl- } \\
\text { sruhe, North Dakota }\end{array}$ & 285 & 91,220 & 1 & $1938-2011$ & 74 & 9,050 & 13,000 & 1999 & 59,370 \\
\hline 54 & 05123400 & $\begin{array}{l}\text { Willow Creek near Willow City, } \\
\text { North Dakota }\end{array}$ & 730 & 250,500 & 1 & $1957-2011$ & 55 & 15,100 & 42,060 & 1999 & 233,800 \\
\hline 56 & 05124000 & $\begin{array}{l}\text { Souris River near Westhope, } \\
\text { North Dakota }\end{array}$ & 6,600 & $3,403,000$ & 1 & $1931-2011$ & 81 & 105,000 & 237,000 & 1976 & $1,231,000$ \\
\hline \multicolumn{12}{|c|}{ Upper Mississippi River Basin (upstream from the mouth of the Ohio River), excluding the Missouri River Basin } \\
\hline 57 & 05211000 & $\begin{array}{l}\text { Mississippi River at Grand } \\
\text { Rapids, Minnesota }\end{array}$ & 3,370 & $1,506,000$ & 11 & $\begin{array}{l}\text { 1886-88, } \\
1901-03, \\
1905-09 \\
1912-37 \\
1939- \\
2011\end{array}$ & 110 & 890,500 & 898,700 & 1906 & $1,759,000$ \\
\hline 58 & 05244000 & $\begin{array}{l}\text { Crow Wing River at Nimrod, } \\
\text { Minnesota }\end{array}$ & 1,030 & 475,600 & 4 & $\begin{array}{c}1940-81 \\
1992- \\
2011\end{array}$ & 62 & 360,500 & 349,100 & 1999 & 548,000 \\
\hline 59 & 05267000 & $\begin{array}{l}\text { Mississippi River near Royal- } \\
\text { ton, Minnesota }\end{array}$ & 11,600 & $5,756,000$ & 6 & $1925-2011$ & 87 & $3,388,000$ & $3,524,000$ & 1966 & $6,921,000$ \\
\hline 60 & 05270500 & $\begin{array}{l}\text { Sauk River near St. Cloud, } \\
\text { Minnesota }\end{array}$ & 1,030 & 752,900 & 1 & $\begin{array}{l}\text { 1910-12, } \\
1931, \\
1935-81, \\
1991- \\
2011\end{array}$ & 72 & 233,800 & 238,300 & 1972 & 530,700 \\
\hline 62 & 05280000 & $\begin{array}{l}\text { Crow River at Rockford, Min- } \\
\text { nesota }\end{array}$ & 2,640 & $2,367,000$ & 1 & $\begin{array}{l}\text { 1910-11, } \\
\text { 1913-17, } \\
1931 \\
1935- \\
2011\end{array}$ & 85 & 521,300 & 632,900 & 1986 & $1,991,000$ \\
\hline 63 & 05286000 & $\begin{array}{l}\text { Rum River near St. Francis, } \\
\text { Minnesota }\end{array}$ & 1,360 & $1,021,000$ & 2 & $\begin{array}{l}1931,1934- \\
2011\end{array}$ & 79 & 461,200 & 471,000 & 1986 & $1,093,000$ \\
\hline
\end{tabular}




\begin{tabular}{|c|c|c|c|c|c|c|c|c|c|}
\hline \multirow{5}{*}{$\begin{array}{c}\text { Estimated } \\
\text { AEP for } \\
\text { observed } \\
2011 \\
\text { annual } \\
\text { runoff } \\
\text { (percent) }\end{array}$} & \multirow{2}{*}{\multicolumn{9}{|c|}{ Expected annual runoff in acre-feet for selected annual exceedance probabilities (AEP) based on Water Resources Council Bulletin 17B methods }} \\
\hline & & & & & & & & & \\
\hline & \multicolumn{3}{|c|}{$\begin{array}{c}\text { 2-percent AEP } \\
\text { (50-year recurrence) }\end{array}$} & \multicolumn{3}{|c|}{$\begin{array}{c}\text { 1-percent AEP } \\
\text { (100-year recurrence) }\end{array}$} & \multicolumn{3}{|c|}{$\begin{array}{c}\text { 0.2-percent AEP } \\
\text { (500-year recurrence) }\end{array}$} \\
\hline & \multirow[b]{2}{*}{ Estimate } & \multicolumn{2}{|c|}{ 95-percent confidence limit } & \multirow[b]{2}{*}{ Estimate } & \multicolumn{2}{|c|}{ 95-percent confidence limit } & \multirow[b]{2}{*}{ Estimate } & \multicolumn{2}{|c|}{ 95-percent confidence limit } \\
\hline & & Low & High & & Low & High & & Low & High \\
\hline \multicolumn{10}{|c|}{ Red River of the North Basin-Continued } \\
\hline 1 to 2 & 864,200 & 621,800 & $1,393,000$ & $1,083,000$ & 724,500 & $1,906,000$ & $1,668,000$ & 918,000 & $3,650,000$ \\
\hline 0.2 to 1 & $10,420,000$ & $8,085,000$ & $14,840,000$ & $12,250,000$ & $8,937,000$ & $18,580,000$ & $16,630,000$ & $10,320,000$ & $29,590,000$ \\
\hline \multicolumn{10}{|c|}{ Souris River Basin } \\
\hline$<0.2$ & 178,200 & 13,440 & 368,300 & 208,900 & 9,836 & 518,600 & 265,400 & 9,729 & $1,131,000$ \\
\hline$<0.2$ & 607,400 & 371,600 & $1,206,000$ & 802,900 & 433,600 & $1,794,000$ & $1,357,000$ & 528,200 & $4,103,000$ \\
\hline 0.2 to 1 & 129,100 & 78,290 & 273,600 & 169,700 & 91,160 & 408,500 & 284,900 & 110,700 & 947,400 \\
\hline$<0.2$ & 755,700 & 500,000 & $1,412,000$ & $1,023,000$ & 622,500 & $2,155,000$ & $1,840,000$ & 894,000 & $5,196,000$ \\
\hline 0.2 to 1 & 63,330 & 42,730 & 120,300 & 80,830 & 50,310 & 172,900 & 129,600 & 64,690 & 369,700 \\
\hline 0.2 to 1 & 201,600 & 5,662 & 500,600 & 221,100 & 3,991 & 744,900 & 247,300 & 16,350 & $2,141,000$ \\
\hline$<0.2$ & $1,465,000$ & 489,400 & $2,795,000$ & $1,837,000$ & 424,500 & $4,004,000$ & $2,679,000$ & 370,600 & $8,517,000$ \\
\hline \multicolumn{10}{|c|}{ Upper Mississippi River Basin (upstream from the mouth of the Ohio River), excluding the Missouri River Basin } \\
\hline 4 to 10 & $1,799,000$ & $1,132,000$ & $2,116,000$ & $1,887,000$ & $1,122,000$ & $2,316,000$ & $2,029,000$ & $1,181,000$ & $2,790,000$ \\
\hline 4 to 10 & 523,900 & 472,400 & 590,100 & 545,900 & 475,400 & 626,900 & 588,100 & 472,600 & 712,600 \\
\hline 4 to 10 & $7,155,000$ & $6,042,000$ & $8,568,000$ & $7,709,000$ & $6,157,000$ & $9,527,000$ & $8,814,000$ & $6,208,000$ & $11,870,000$ \\
\hline 0.2 to 1 & 595,600 & 461,400 & 779,000 & 658,300 & 467,100 & 901,200 & 787,600 & 460,000 & $1,224,000$ \\
\hline
\end{tabular}

\begin{tabular}{|c|c|c|c|c|c|c|c|c|c|}
\hline 1 to 2 & $2,079,000$ & $1,524,000$ & $2,927,000$ & $2,392,000$ & $1,587,000$ & $3,575,000$ & $3,084,000$ & $1,624,000$ & $5,416,000$ \\
\hline 0.2 to 1 & 943,700 & 541,700 & $1,131,000$ & 992,700 & 509,700 & $1,245,000$ & $1,073,000$ & 546,600 & $1,527,000$ \\
\hline
\end{tabular}


Table 2. Annual runoff data and results of flood-probability analyses for selected streamgages in the Central United States affected by 2011 flooding.-Continued

[Stations used in the trend analysis are italicized. mi $^{2}$, square miles; AEP, annual exceedance probability; <, less than; >, greater than]

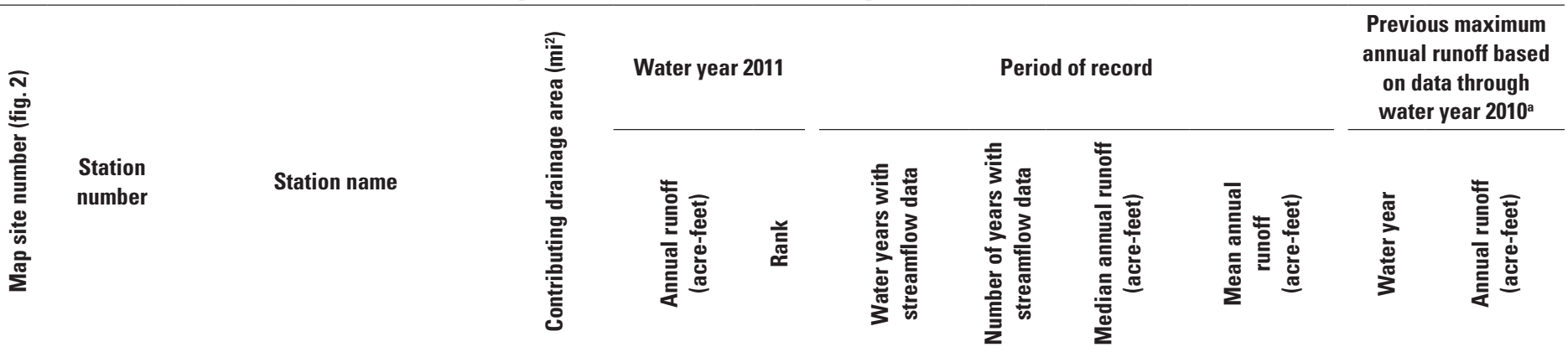

Upper Mississippi River Basin (upstream from the mouth of the Ohio River), excluding the Missouri River Basin-Continued

\begin{tabular}{|c|c|c|c|c|c|c|c|c|c|c|c|}
\hline 65 & 05288500 & $\begin{array}{l}\text { Mississippi River near Anoka, } \\
\text { Minnesota }\end{array}$ & 19,100 & $11,730,000$ & 2 & $1932-2011$ & 80 & $5,998,000$ & $6,064,000$ & 1986 & $12,810,000$ \\
\hline 67 & 05292000 & $\begin{array}{l}\text { Minnesota River at Ortonville, } \\
\text { Minnesota }\end{array}$ & 1,160 & 530,700 & 1 & 1939-2011 & 73 & 72,400 & 112,600 & 2010 & 453,200 \\
\hline 69 & 05294000 & $\begin{array}{c}\text { Pomme De Terre River at } \\
\text { Appleton, Minnesota }\end{array}$ & 905 & 490,100 & 1 & $\begin{array}{l}\text { 1936-99, } \\
2004-11\end{array}$ & 72 & 78,190 & 107,900 & 1997 & 304,100 \\
\hline 70 & 05300000 & $\begin{array}{c}\text { Lac Qui Parle River near Lac } \\
\text { Qui Parle, Minnesota }\end{array}$ & 960 & 553,100 & 1 & $\begin{array}{c}1913,1932 \\
1934-99 \\
2002-11\end{array}$ & 78 & 92,670 & 125,900 & 1993 & 452,500 \\
\hline 72 & 05311000 & $\begin{array}{l}\text { Minnesota River at Montevi- } \\
\text { deo, Minnesota }\end{array}$ & 6,180 & $3,337,000$ & 1 & $\begin{array}{c}1910-17, \\
1930- \\
2011\end{array}$ & 90 & 530,700 & 727,400 & 1997 & $2,295,000$ \\
\hline 76 & 05317000 & $\begin{array}{l}\text { Cottonwood River near New } \\
\text { Ulm, Minnesota }\end{array}$ & 1,300 & $1,187,000$ & 2 & $\begin{array}{l}1912-13 \\
1936-37 \\
1939- \\
2011\end{array}$ & 77 & 202,700 & 298,100 & 1993 & $1,303,000$ \\
\hline 78 & 05320000 & $\begin{array}{l}\text { Blue Earth River near Rapi- } \\
\quad \text { dan, Minnesota }\end{array}$ & 2,410 & $2,186,000$ & 2 & $\begin{array}{c}1940-45 \\
1950- \\
2011\end{array}$ & 68 & 721,800 & 827,500 & 1993 & $3,272,000$ \\
\hline 81 & 05320500 & $\begin{array}{l}\text { Le Sueur River near Rapidan, } \\
\quad \text { Minnesota }\end{array}$ & 1,110 & $1,035,000$ & 3 & $\begin{array}{c}1940-45 \\
1950- \\
2011\end{array}$ & 68 & 413,400 & 420,600 & 1993 & $1,477,000$ \\
\hline 82 & 05325000 & $\begin{array}{l}\text { Minnesota River at Mankato, } \\
\text { Minnesota }\end{array}$ & 14,900 & $11,000,000$ & 1 & $\begin{array}{l}1905 \\
1911-17 \\
1930- \\
2011\end{array}$ & 90 & $2,237,000$ & $2,801,000$ & 1993 & $10,790,000$ \\
\hline 83 & 05330000 & $\begin{array}{l}\text { Minnesota River near Jordan, } \\
\text { Minnesota }\end{array}$ & 16,200 & $12,740,000$ & 1 & $1935-2011$ & 77 & $2,744,000$ & $3,476,000$ & 1993 & $12,240,000$ \\
\hline 84 & 05331000 & $\begin{array}{l}\text { Mississippi River at Saint Paul, } \\
\text { Minnesota }\end{array}$ & 36,800 & $26,500,000$ & 1 & $\begin{array}{c}1895,1897 \\
1901-05 \\
1907- \\
2011\end{array}$ & 112 & $8,579,000$ & $8,845,000$ & 1986 & $21,430,000$ \\
\hline 85 & 05340500 & $\begin{array}{l}\text { St. Croix River at St. Croix } \\
\text { Falls, Wisconsin }\end{array}$ & 6,240 & $4,793,000$ & 5 & $1911-2011$ & 101 & $3,178,000$ & $3,175,000$ & 1986 & $6,204,000$ \\
\hline 86 & 05344500 & $\begin{array}{l}\text { Mississippi River at Prescott, } \\
\text { Wisconsin }\end{array}$ & 44,800 & $31,560,000$ & 1 & 1929-2011 & 83 & $13,830,000$ & $13,600,000$ & 1986 & $27,870,000$ \\
\hline 88 & 05355200 & $\begin{array}{l}\text { Cannon River at Welch, Min- } \\
\text { nesota }\end{array}$ & 1,340 & $1,281,000$ & 2 & $\begin{array}{l}\text { 1912-13, } \\
1932-71, \\
1992- \\
2011\end{array}$ & 62 & 431,100 & 479,600 & 1993 & $1,542,000$ \\
\hline 89 & 05369500 & $\begin{array}{l}\text { Chippewa River at Durand, } \\
\text { Wisconsin }\end{array}$ & 9,010 & $7,030,000$ & 10 & 1929-2011 & 83 & $5,640,000$ & $5,540,000$ & 1942 & $8,398,000$ \\
\hline 90 & 05378500 & $\begin{array}{l}\text { Mississippi River at Winona, } \\
\text { Minnesota }\end{array}$ & 59,200 & $42,860,000$ & 1 & 1929-2011 & 83 & $21,570,000$ & $21,650,000$ & 1986 & $41,120,000$ \\
\hline 91 & 05382000 & $\begin{array}{l}\text { Black River near Galesville, } \\
\quad \text { Wisconsin }\end{array}$ & 2,080 & $1,832,000$ & 9 & 1933-2011 & 79 & $1,303,000$ & $1,283,000$ & 1993 & $2,505,000$ \\
\hline
\end{tabular}


Expected annual runoff in acre-feet for selected annual exceedance probabilities (AEP) based on Water Resources Council Bulletin 17B methods

Estimated

AEP for

observed

2011

annual

runoff

(percent)

\begin{tabular}{c}
$\begin{array}{c}\text { 2-percent AEP } \\
\text { (50-year recurrence) }\end{array}$ \\
\hline 95-percent confidence limit \\
\hline
\end{tabular}

Estimate
Low High Estimate 1-percent AEP

(100-year recurrence)

95-percent confidence limit

Low High 0.2-percent AEP

(500-year recurrence)

95-percent confidence limit

Estimate

Upper Mississippi River Basin (upstream from the mouth of the Ohio River), excluding the Missouri River Basin-Continued

\begin{tabular}{|c|c|c|c|c|c|c|c|c|c|}
\hline 1 to 2 & $11,520,000$ & $8,276,000$ & $13,500,000$ & $12,130,000$ & $7,917,000$ & $14,730,000$ & $13,210,000$ & $7,763,000$ & $17,690,000$ \\
\hline 1 to 2 & 478,100 & 247,500 & 758,300 & 561,800 & 228,900 & 972,300 & 740,000 & 203,100 & $1,646,000$ \\
\hline 0.2 to 1 & 382,900 & 285,600 & 666,100 & 466,800 & 331,300 & 918,000 & 694,100 & 431,000 & $1,825,000$ \\
\hline 1 to 2 & 520,300 & 366,800 & 844,700 & 629,200 & 403,400 & $1,107,000$ & 897,300 & 450,200 & $1,949,000$ \\
\hline$<0.2$ & $2,142,000$ & 679,200 & $3,033,000$ & $2,308,000$ & 587,800 & $3,607,000$ & $2,563,000$ & 778,100 & $5,259,000$ \\
\hline 2 to 4 & $1,202,000$ & 871,700 & $2,145,000$ & $1,498,000$ & $1,027,000$ & $3,023,000$ & $2,321,000$ & $1,367,000$ & $6,275,000$ \\
\hline 2 to 4 & $2,471,000$ & $1,644,000$ & $3,443,000$ & $2,774,000$ & $1,584,000$ & $4,105,000$ & $3,393,000$ & $1,462,000$ & $5,950,000$ \\
\hline 4 to 10 & $1,190,000$ & 623,200 & $1,614,000$ & $1,308,000$ & 560,100 & $1,892,000$ & $1,528,000$ & 531,900 & $2,659,000$ \\
\hline 0.2 to 1 & $8,759,000$ & $6,073,000$ & $11,900,000$ & $9,905,000$ & $6,031,000$ & $14,260,000$ & $12,260,000$ & $5,828,000$ & $20,730,000$ \\
\hline
\end{tabular}

\begin{tabular}{llllllllll}
0.2 to 1 & $10,850,000$ & $8,433,000$ & $16,170,000$ & $12,680,000$ & $9,346,000$ & $20,320,000$ & $17,130,000$ & $10,920,000$ & $32,810,000$ \\
\hline 0.2 to 1 & $21,130,000$ & $17,890,000$ & $26,080,000$ & $23,430,000$ & $18,970,000$ & $30,020,000$ & $28,380,000$ & $20,530,000$ & $40,090,000$
\end{tabular}

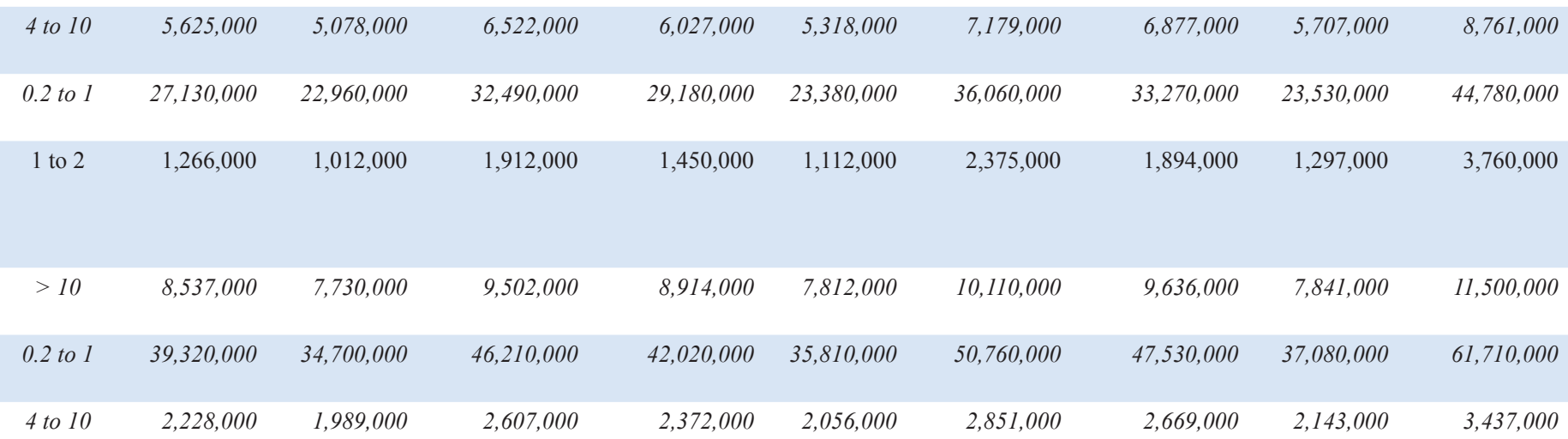




\section{Annual Exceedance Probabilities and Trends for the Central United States during the 2011 Floods}

Table 2. Annual runoff data and results of flood-probability analyses for selected streamgages in the Central United States affected by 2011 flooding.-Continued

[Stations used in the trend analysis are italicized. $\mathrm{mi}^{2}$, square miles; AEP, annual exceedance probability; <, less than; >, greater than]

\begin{tabular}{|c|c|c|c|c|c|c|c|c|c|c|c|}
\hline \multirow{2}{*}{ 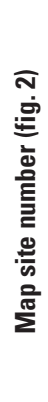 } & \multirow[b]{2}{*}{$\begin{array}{l}\text { Station } \\
\text { number }\end{array}$} & \multirow[b]{2}{*}{ Station name } & \multirow{2}{*}{ 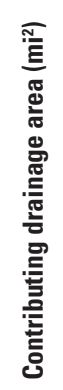 } & \multicolumn{2}{|c|}{ Water year 2011} & \multicolumn{4}{|c|}{ Period of record } & \multicolumn{2}{|c|}{$\begin{array}{l}\text { Previous maximum } \\
\text { annual runoff based } \\
\text { on data through } \\
\text { water year } 2010^{\mathrm{a}}\end{array}$} \\
\hline & & & & 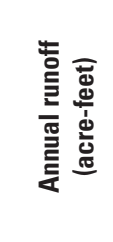 & 恙 & 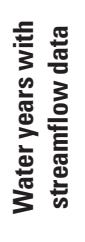 & 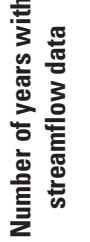 & 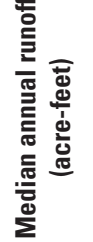 & 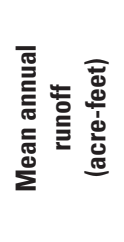 & 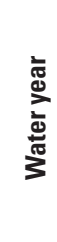 & 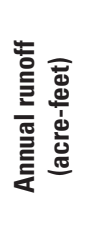 \\
\hline
\end{tabular}

\begin{tabular}{|c|c|c|c|c|c|c|c|c|c|c|c|}
\hline \multicolumn{12}{|c|}{ Upper Mississippi River Basin (upstream from the mouth of the Ohio River), excluding the Missouri River Basin-Continued } \\
\hline 92 & 05389500 & $\begin{array}{l}\text { Mississippi River at McGregor, } \\
\quad \text { Iowa }\end{array}$ & 67,500 & $49,950,000$ & 1 & $\begin{array}{r}1937-2005 \\
2008-11\end{array}$ & 73 & $28,020,000$ & $27,330,000$ & 1993 & $46,840,000$ \\
\hline 94 & 05398000 & $\begin{array}{l}\text { Wisconsin River at Rothschild, } \\
\text { Wisconsin }\end{array}$ & 4,020 & $3,077,000$ & 15 & $1945-2011$ & 67 & $2,490,000$ & $2,511,000$ & 1973 & $4,308,000$ \\
\hline 95 & 05407000 & $\begin{array}{l}\text { Wisconsin River at Muscoda, } \\
\text { Wisconsin }\end{array}$ & 10,400 & $8,326,000$ & 8 & $1914-2011$ & 98 & $6,277,000$ & $6,293,000$ & 1973 & $11,580,000$ \\
\hline 96 & 05412500 & Turkey River at Garber, Iowa & 1,545 & $1,057,000$ & 18 & $\begin{array}{l}1914-16 \\
\quad 1920-27 \\
1930- \\
2011\end{array}$ & 91 & 718,900 & 754,900 & 1993 & $2,107,000$ \\
\hline 102 & 05420500 & $\begin{array}{l}\text { Mississippi River at Clinton, } \\
\text { Iowa }\end{array}$ & 85,600 & $65,300,000$ & 3 & $1874-2011$ & 138 & $35,730,000$ & $35,720,000$ & 1882 & $68,560,000$ \\
\hline 103 & 05422000 & $\begin{array}{l}\text { Wapsipinicon River near De } \\
\text { Witt, Iowa }\end{array}$ & 2,336 & $1,643,000$ & 21 & $1935-2011$ & 77 & $1,158,000$ & $1,267,000$ & 1993 & $3,953,000$ \\
\hline 104 & 05430500 & Rock River at Afton, Wisconsin & 3,340 & $2,208,000$ & 13 & $1915-2011$ & 97 & $1,376,000$ & $1,460,000$ & 2008 & $3,598,000$ \\
\hline 105 & 05437500 & Rock River at Rockton, Illinois & 6,363 & $4,213,000$ & 16 & $\begin{array}{l}\text { 1904-05 } \\
1915-19 \\
1940- \\
2011\end{array}$ & 79 & $3,026,000$ & $3,254,000$ & 2008 & $7,124,000$ \\
\hline 106 & 05446500 & Rock River near Joslin, Illinois & 9,549 & $6,581,000$ & 14 & $1940-2011$ & 72 & $4,608,000$ & $4,973,000$ & 1993 & $10,570,000$ \\
\hline 108 & 05454500 & Iowa River at Iowa City, Iowa & 3,271 & $1,911,000$ & 25 & $1904-2011$ & 108 & $1,256,000$ & $1,425,000$ & 1993 & $6,154,000$ \\
\hline 109 & 05458500 & $\begin{array}{l}\text { Cedar River at Janesville, } \\
\quad \text { Iowa }\end{array}$ & 1,661 & $1,325,000$ & 8 & $\begin{array}{l}\text { 1905-06, } \\
1915-27, \\
1933-42, \\
1946- \\
2011\end{array}$ & 91 & 626,200 & 729,000 & 1993 & $2,498,000$ \\
\hline 110 & 05464500 & $\begin{array}{l}\text { Cedar River at Cedar Rapids, } \\
\quad \text { Iowa }\end{array}$ & 6,510 & $4,742,000$ & 10 & $1903-2011$ & 109 & $2,585,000$ & $2,873,000$ & 1993 & $10,930,000$ \\
\hline 112 & 05465500 & Iowa River at Wapello, Iowa & 12,500 & $8,326,000$ & 18 & $1915-2011$ & 97 & $5,263,000$ & $5,809,000$ & 1993 & $22,150,000$ \\
\hline 116 & 05476000 & $\begin{array}{l}\text { Des Moines River at Jackson, } \\
\text { Minnesota }\end{array}$ & 1,250 & $1,267,000$ & 2 & $1936-2011$ & 76 & 216,500 & 307,600 & 1993 & $1,520,000$ \\
\hline 117 & 05479000 & $\begin{array}{l}\text { East Fork Des Moines River at } \\
\text { Dakota City, Iowa }\end{array}$ & 1,308 & 955,600 & 7 & $1941-2011$ & 71 & 396,700 & 481,400 & 1993 & $1,984,000$ \\
\hline 118 & 05480500 & $\begin{array}{l}\text { Des Moines River at Fort } \\
\text { Dodge, Iowa }\end{array}$ & 4,190 & $3,417,000$ & 5 & $\begin{array}{c}1914-27 \\
1947- \\
2011\end{array}$ & 79 & $1,173,000$ & $1,374,000$ & 1993 & $5,705,000$ \\
\hline 119 & 05484500 & $\begin{array}{l}\text { Raccoon River at Van Meter, } \\
\quad \text { Iowa }\end{array}$ & 3,441 & $1,622,000$ & 22 & $1916-2011$ & 96 & 962,900 & $1,191,000$ & 1993 & $4,141,000$ \\
\hline 120 & 05485500 & $\begin{array}{l}\text { Des Moines River below Ra- } \\
\text { coon River at Des Moines, } \\
\text { Iowa }\end{array}$ & 9,879 & $6,277,000$ & 8 & $1941-2011$ & 71 & $3,461,000$ & $3,851,000$ & 1993 & $13,900,000$ \\
\hline 125 & 05490500 & $\begin{array}{l}\text { Des Moines River at Keosau- } \\
\quad \text { qua, Iowa }\end{array}$ & 14,038 & $9,846,000$ & 7 & $\begin{array}{c}1904-05 \\
1912- \\
2011\end{array}$ & 102 & $4,130,000$ & $4,971,000$ & 1993 & $19,470,000$ \\
\hline 128 & 05526000 & $\begin{array}{l}\text { Iroquois River near Chen- } \\
\quad \text { banse, Illinois }\end{array}$ & 2,091 & $1,332,000$ & 40 & 1924-2011 & 88 & $1,274,000$ & $1,303,000$ & 1993 & $3,243,000$ \\
\hline
\end{tabular}


Expected annual runoff in acre-feet for selected annual exceedance probabilities (AEP) based on Water Resources Council Bulletin 17B methods

\begin{tabular}{|c|c|c|c|c|c|c|c|c|c|}
\hline \multirow{3}{*}{$\begin{array}{l}\text { Estimated } \\
\text { AEP for } \\
\text { observed } \\
2011 \\
\text { annual } \\
\text { runoff } \\
\text { (percent) }\end{array}$} & \multicolumn{3}{|c|}{$\begin{array}{c}\text { 2-percent AEP } \\
\text { (50-year recurrence) }\end{array}$} & \multicolumn{3}{|c|}{$\begin{array}{c}\text { 1-percent AEP } \\
\text { (100-year recurrence) }\end{array}$} & \multicolumn{3}{|c|}{$\begin{array}{c}\text { 0.2-percent AEP } \\
\text { (500-year recurrence) }\end{array}$} \\
\hline & \multirow[b]{2}{*}{ Estimate } & \multicolumn{2}{|c|}{ 95-percent confidence limit } & \multirow[b]{2}{*}{ Estimate } & \multicolumn{2}{|c|}{ 95-percent confidence limit } & \multirow[b]{2}{*}{ Estimate } & \multicolumn{2}{|c|}{ 95-percent confidence limit } \\
\hline & & Low & High & & Low & High & & Low & High \\
\hline \multicolumn{10}{|c|}{ Upper Mississippi River Basin (upstream from the mouth of the Ohio River), excluding the Missouri River Basin-Continued } \\
\hline 0.2 to 1 & $46,060,000$ & $41,410,000$ & $54,260,000$ & $49,010,000$ & $43,020,000$ & $59,430,000$ & $55,200,000$ & $45,400,000$ & $71,900,000$ \\
\hline$>10$ & $4,050,000$ & $3,657,000$ & $4,717,000$ & $4,279,000$ & $3,768,000$ & $5,113,000$ & $4,748,000$ & $3,912,000$ & $6,058,000$ \\
\hline 4 to 10 & $9,566,000$ & $8,835,000$ & $10,560,000$ & $10,010,000$ & $9,036,000$ & $11,240,000$ & $10,870,000$ & $9,284,000$ & $12,780,000$ \\
\hline$>10$ & $1,678,000$ & $1,431,000$ & $2,097,000$ & $1,850,000$ & $1,517,000$ & $2,403,000$ & $2,222,000$ & $1,644,000$ & $3,190,000$ \\
\hline 0.2 to 1 & $58,070,000$ & $53,560,000$ & $64,230,000$ & $61,300,000$ & $55,360,000$ & $69,060,000$ & $67,800,000$ & $58,020,000$ & $80,080,000$ \\
\hline$>10$ & $2,939,000$ & $2,386,000$ & $3,735,000$ & $3,227,000$ & $2,451,000$ & $4,272,000$ & $3,826,000$ & $2,484,000$ & $5,655,000$ \\
\hline$>10$ & $2,893,000$ & $2,515,000$ & $3,428,000$ & $3,121,000$ & $2,606,000$ & $3,809,000$ & $3,588,000$ & $2,711,000$ & $4,735,000$ \\
\hline$>10$ & $6,344,000$ & $5,552,000$ & $7,845,000$ & $6,903,000$ & $5,877,000$ & $8,878,000$ & $8,126,000$ & $6,416,000$ & $11,500,000$ \\
\hline$>10$ & $10,030,000$ & $8,670,000$ & $12,840,000$ & $10,990,000$ & $9,239,000$ & $14,760,000$ & $13,140,000$ & $10,230,000$ & $19,770,000$ \\
\hline$>10$ & $3,716,000$ & $3,016,000$ & $4,694,000$ & $4,147,000$ & $3,158,000$ & $5,463,000$ & $5,061,000$ & $3,316,000$ & $7,455,000$ \\
\hline 4 to 10 & $1,844,000$ & $1,531,000$ & $2,424,000$ & $2,078,000$ & $1,655,000$ & $2,870,000$ & $2,611,000$ & $1,859,000$ & $4,078,000$ \\
\hline
\end{tabular}

\begin{tabular}{|c|c|c|c|c|c|c|c|c|c|}
\hline$>10$ & $7,120,000$ & $6,006,000$ & $8,992,000$ & $7,978,000$ & $6,461,000$ & $10,510,000$ & $9,894,000$ & $7,198,000$ & $14,500,000$ \\
\hline$>10$ & $13,790,000$ & $11,370,000$ & $17,170,000$ & $15,200,000$ & $11,810,000$ & $19,660,000$ & $18,130,000$ & $12,240,000$ & $26,000,000$ \\
\hline 1 to 2 & $1,228,000$ & 805,300 & $1,907,000$ & $1,454,000$ & 825,200 & $2,435,000$ & $1,967,000$ & 811,300 & $4,061,000$ \\
\hline$>10$ & $1,379,000$ & 781,200 & $1,872,000$ & $1,524,000$ & 713,400 & $2,202,000$ & $1,798,000$ & 664,600 & $3,112,000$ \\
\hline 4 to 10 & $4,248,000$ & $3,094,000$ & $5,915,000$ & $4,835,000$ & $3,163,000$ & $7,130,000$ & $6,099,000$ & $3,143,000$ & $10,530,000$ \\
\hline$>10$ & $3,446,000$ & $2,734,000$ & $4,609,000$ & $3,924,000$ & $2,919,000$ & $5,522,000$ & $4,993,000$ & $3,159,000$ & $8,023,000$ \\
\hline$>10$ & $9,845,000$ & $5,714,000$ & $12,840,000$ & $10,710,000$ & $5,242,000$ & $14,780,000$ & $12,310,000$ & $4,999,000$ & $19,940,000$ \\
\hline 4 to 10 & $13,310,000$ & $10,460,000$ & $16,990,000$ & $14,840,000$ & $10,780,000$ & $19,800,000$ & $18,020,000$ & $10,990,000$ & $27,130,000$ \\
\hline$>10$ & $2,443,000$ & $1,558,000$ & $2,849,000$ & $2,556,000$ & $1,521,000$ & $3,097,000$ & $2,741,000$ & $1,583,000$ & $3,693,000$ \\
\hline
\end{tabular}


Table 2. Annual runoff data and results of flood-probability analyses for selected streamgages in the Central United States affected by 2011 flooding.-Continued

[Stations used in the trend analysis are italicized. $\mathrm{mi}^{2}$, square miles; AEP, annual exceedance probability; $<$, less than; $>$, greater than]

\begin{tabular}{|c|c|c|c|c|c|c|c|c|c|c|c|}
\hline \multirow{2}{*}{ 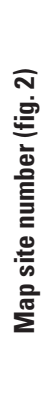 } & \multirow[b]{2}{*}{$\begin{array}{l}\text { Station } \\
\text { number }\end{array}$} & \multirow[b]{2}{*}{ Station name } & \multirow{2}{*}{ 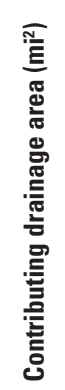 } & \multicolumn{2}{|c|}{ Water year 2011} & \multicolumn{4}{|c|}{ Period of record } & \multicolumn{2}{|c|}{$\begin{array}{c}\text { Previous maximum } \\
\text { annual runoff base } \\
\text { on data through } \\
\text { water year } 2010^{\text {a }}\end{array}$} \\
\hline & & & & 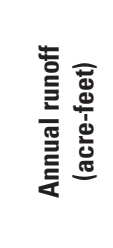 & 兰 & 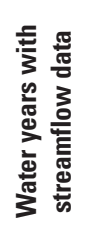 & 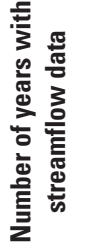 & 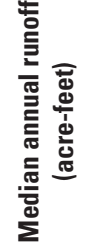 & 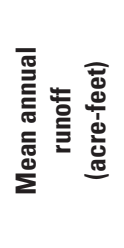 & & 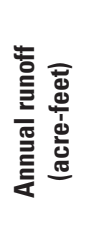 \\
\hline
\end{tabular}

\begin{tabular}{|c|c|c|c|c|c|c|c|c|c|c|c|}
\hline \multicolumn{12}{|c|}{ Upper Mississippi River Basin (upstream from the mouth of the Ohio River), excluding the Missouri River Basin-Continued } \\
\hline 129 & 05527500 & $\begin{array}{l}\text { Kankakee River near Wilming- } \\
\quad \text { ton, Illinois }\end{array}$ & 5,150 & $3,547,000$ & 39 & $\begin{array}{c}1916-33 \\
1936- \\
2011\end{array}$ & 94 & $3,189,000$ & $3,316,000$ & 1993 & $7,529,000$ \\
\hline 130 & 05543500 & $\begin{array}{l}\text { Illinois River at Marseilles, } \\
\text { Illinois }\end{array}$ & 8,259 & $8,108,000$ & 35 & $1920-2011$ & 92 & $7,674,000$ & $7,817,000$ & 1993 & $12,960,000$ \\
\hline 131 & 05552500 & Fox River at Dayton, Illinois & 2,642 & $1,897,000$ & 13 & 1916-2011 & 96 & $1,372,000$ & $1,366,000$ & 1993 & $2,852,000$ \\
\hline 132 & 05555300 & $\begin{array}{l}\text { Vermilion River near Leonore, } \\
\text { Illinois }\end{array}$ & 1,251 & 691,400 & 32 & $1932-2011$ & 80 & 628,100 & 653,300 & 1993 & $1,752,000$ \\
\hline 133 & 05568500 & $\begin{array}{l}\text { Illinois River at Kingston } \\
\text { Mines, Illinois }\end{array}$ & 15,818 & $13,470,000$ & 20 & $1940-2011$ & 72 & $11,440,000$ & $11,640,000$ & 1993 & $23,310,000$ \\
\hline 134 & 05570000 & Spoon River at Seville, Illinois & 1,636 & $1,187,000$ & 16 & $1915-2011$ & 97 & 760,200 & 825,500 & 1993 & $2,592,000$ \\
\hline 135 & 05576500 & $\begin{array}{l}\text { Sangamon River at Riverton, } \\
\text { Illinois }\end{array}$ & 2,618 & $1,962,000$ & 15 & $\begin{array}{l}\text { 1909-12, } \\
1915-56, \\
1987- \\
2011\end{array}$ & 71 & $1,318,000$ & $1,332,000$ & 1927 & $3,482,000$ \\
\hline 136 & 05582000 & $\begin{array}{l}\text { Salt Creek near Greenview, } \\
\text { Illinois }\end{array}$ & 1,804 & $1,071,000$ & 31 & $1942-2011$ & 70 & 984,600 & $1,020,000$ & 1993 & $2,433,000$ \\
\hline 137 & 05583000 & $\begin{array}{l}\text { Sangamon River near Oakford, } \\
\text { Illinois }\end{array}$ & 5,093 & $3,497,000$ & 22 & $\begin{array}{l}\text { 1910-11, } \\
1915-18, \\
1922, \\
1929-33, \\
1940- \\
2011\end{array}$ & 84 & $2,563,000$ & $2,624,000$ & 2010 & $6,545,000$ \\
\hline 138 & 05586100 & $\begin{array}{l}\text { Illinois River at Valley City, } \\
\text { Illinois }\end{array}$ & 26,743 & $21,140,000$ & 15 & 1939-2011 & 73 & $16,290,000$ & $16,970,000$ & 1993 & $33,880,000$ \\
\hline 141 & 05592500 & $\begin{array}{l}\text { Kaskaskia River at Vandalia, } \\
\text { Illinois }\end{array}$ & 1,940 & $1,759,000$ & 13 & $\begin{array}{c}1909-12 \\
1915- \\
2011\end{array}$ & 101 & $1,108,000$ & $1,132,000$ & 1927 & $2,614,000$ \\
\hline 143 & 05595000 & $\begin{array}{l}\text { Kaskaskia River at New Ath- } \\
\text { ens, Illinois }\end{array}$ & 5,189 & $4,887,000$ & 3 & $\begin{array}{c}1910-12, \\
1915-21, \\
1935-71, \\
2010-11\end{array}$ & 49 & $2,795,000$ & $2,728,000$ & 1950 & $5,893,000$ \\
\hline 144 & 05597000 & $\begin{array}{l}\text { Big Muddy River at Plumfield, } \\
\text { Illinois }\end{array}$ & 794 & 991,800 & 4 & $\begin{array}{c}1909,1912 \\
1915- \\
2011\end{array}$ & 99 & 544,400 & 523,100 & 1950 & $1,325,000$ \\
\hline 146 & 07010000 & $\begin{array}{l}\text { Mississippi River at St. Louis, } \\
\text { Missouri }\end{array}$ & 697,000 & $233,800,000$ & 5 & $1863-2011$ & 149 & $131,800,000$ & $135,500,000$ & 1993 & $311,300,000$ \\
\hline 147 & 07019000 & $\begin{array}{l}\text { Meramec River near Eureka, } \\
\quad \text { Missouri }\end{array}$ & 3,788 & $3,026,000$ & 23 & $\begin{array}{c}1904-05 \\
1922- \\
2011\end{array}$ & 92 & $2,299,000$ & $2,379,000$ & 1985 & $5,365,000$ \\
\hline 148 & 07020500 & $\begin{array}{l}\text { Mississippi River at Chester, } \\
\text { Illinois }\end{array}$ & 708,600 & $244,700,000$ & 4 & $1943-2011$ & 69 & $145,500,000$ & $153,200,000$ & 1993 & $320,000,000$ \\
\hline 149 & 07022000 & $\begin{array}{l}\text { Mississippi River at Thebes, } \\
\text { Illinois }\end{array}$ & 713,200 & $260,600,000$ & 3 & $\begin{array}{c}1934-37 \\
1940- \\
2011\end{array}$ & 76 & $148,400,000$ & $154,100,000$ & 1993 & $322,900,000$ \\
\hline
\end{tabular}




\begin{tabular}{|c|c|c|c|c|c|c|c|c|c|}
\hline \multirow{4}{*}{$\begin{array}{c}\text { Estimated } \\
\text { AEP for } \\
\text { observed } \\
2011 \\
\text { annual } \\
\text { runoff } \\
\text { (percent) }\end{array}$} & \multicolumn{9}{|c|}{ Expected annual runoff in acre-feet for selected annual exceedance probabilities (AEP) based on Water Resources Council Bulletin 17B methods } \\
\hline & \multicolumn{3}{|c|}{$\begin{array}{c}\text { 2-percent AEP } \\
\text { (50-year recurrence) }\end{array}$} & \multicolumn{3}{|c|}{$\begin{array}{c}\text { 1-percent AEP } \\
\text { (100-year recurrence) }\end{array}$} & \multicolumn{3}{|c|}{$\begin{array}{c}\text { 0.2-percent AEP } \\
\text { (500-year recurrence) }\end{array}$} \\
\hline & \multirow[b]{2}{*}{ Estimate } & \multicolumn{2}{|c|}{ 95-percent confidence limit } & \multirow[b]{2}{*}{ Estimate } & \multicolumn{2}{|c|}{ 95-percent confidence limit } & \multirow[b]{2}{*}{ Estimate } & \multicolumn{2}{|c|}{ 95-percent confidence limit } \\
\hline & & Low & High & & Low & High & & Low & High \\
\hline \multicolumn{10}{|c|}{ Upper Mississippi River Basin (upstream from the mouth of the Ohio River), excluding the Missouri River Basin-Continued } \\
\hline$>10$ & $6,250,000$ & $5,438,000$ & $7,290,000$ & $6,681,000$ & $5,564,000$ & $8,008,000$ & $7,540,000$ & $5,668,000$ & $9,721,000$ \\
\hline$>10$ & $12,050,000$ & $11,150,000$ & $13,610,000$ & $12,720,000$ & $11,580,000$ & $14,700,000$ & $14,100,000$ & $12,310,000$ & $17,240,000$ \\
\hline$>10$ & $2,671,000$ & $2,246,000$ & $3,124,000$ & $2,853,000$ & $2,260,000$ & $3,436,000$ & $3,204,000$ & $2,247,000$ & $4,182,000$ \\
\hline$>10$ & $1,423,000$ & 769,100 & $1,746,000$ & $1,509,000$ & 737,600 & $1,947,000$ & $1,655,000$ & 779,900 & $2,455,000$ \\
\hline$>10$ & $19,930,000$ & $17,800,000$ & $23,500,000$ & $21,210,000$ & $18,430,000$ & $25,720,000$ & $23,860,000$ & $19,250,000$ & $31,080,000$ \\
\hline$>10$ & $1,925,000$ & $1,557,000$ & $2,369,000$ & $2,105,000$ & $1,584,000$ & $2,690,000$ & $2,467,000$ & $1,592,000$ & $3,495,000$ \\
\hline$>10$ & $2,969,000$ & $1,267,000$ & $3,794,000$ & $3,123,000$ & $1,141,000$ & $4,275,000$ & $3,358,000$ & $1,318,000$ & $5,585,000$ \\
\hline$>10$ & $2,289,000$ & $1,901,000$ & $2,957,000$ & $2,518,000$ & $1,988,000$ & $3,394,000$ & $3,009,000$ & $2,086,000$ & $4,533,000$ \\
\hline$>10$ & $5,788,000$ & $4,159,000$ & $7,058,000$ & $6,212,000$ & $3,997,000$ & $7,893,000$ & $6,993,000$ & $3,841,000$ & $9,975,000$ \\
\hline$>10$ & $30,810,000$ & $27,150,000$ & $36,960,000$ & $33,020,000$ & $28,190,000$ & $40,850,000$ & $37,630,000$ & $29,540,000$ & $50,380,000$ \\
\hline$>10$ & $1,950,000$ & 892,700 & $2,521,000$ & $1,974,000$ & $1,107,000$ & $2,784,000$ & $1,997,000$ & $1,317,000$ & $3,553,000$ \\
\hline 4 to 10 & $5,281,000$ & $1,552,000$ & $7,156,000$ & $5,412,000$ & $1,396,000$ & $8,164,000$ & $5,567,000$ & $2,435,000$ & $11,900,000$ \\
\hline 2 to 4 & 995,700 & 456,000 & $1,262,000$ & $1,019,000$ & 473,500 & $1,395,000$ & $1,046,000$ & 611,200 & $1,769,000$ \\
\hline 2 to 4 & $245,000,000$ & $223,500,000$ & $279,100,000$ & $263,100,000$ & $235,300,000$ & $307,200,000$ & $301,900,000$ & $256,200,000$ & $374,700,000$ \\
\hline$>10$ & $5,057,000$ & $4,331,000$ & $6,168,000$ & $5,515,000$ & $4,526,000$ & $6,962,000$ & $6,479,000$ & $4,772,000$ & $8,948,000$ \\
\hline 4 to 10 & $275,400,000$ & $243,800,000$ & $344,200,000$ & $297,900,000$ & $258,200,000$ & $390,000,000$ & $348,100,000$ & $284,500,000$ & $508,300,000$ \\
\hline 2 to 4 & $268,900,000$ & $237,400,000$ & $316,100,000$ & $286,300,000$ & $243,900,000$ & $345,900,000$ & $321,600,000$ & $250,500,000$ & $417,500,000$ \\
\hline
\end{tabular}


Table 2. Annual runoff data and results of flood-probability analyses for selected streamgages in the Central United States affected by 2011 flooding.-Continued

[Stations used in the trend analysis are italicized. $\mathrm{mi}^{2}$, square miles; AEP, annual exceedance probability; <, less than; >, greater than]

\begin{tabular}{|c|c|c|c|c|c|c|c|c|c|c|c|}
\hline \multirow{2}{*}{ 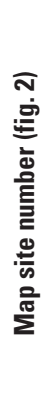 } & \multirow[b]{2}{*}{$\begin{array}{l}\text { Station } \\
\text { number }\end{array}$} & \multirow[b]{2}{*}{ Station name } & \multirow{2}{*}{ 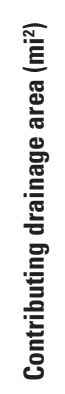 } & \multicolumn{2}{|c|}{ Water year 2011} & \multicolumn{4}{|c|}{ Period of record } & \multicolumn{2}{|c|}{$\begin{array}{c}\text { Previous maximun } \\
\text { annual runoff base } \\
\text { on data through } \\
\text { water year } 2010^{\text {a }}\end{array}$} \\
\hline & & & & 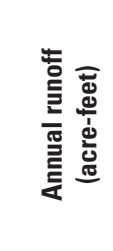 & 兰 & 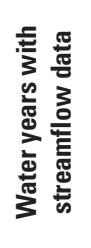 & 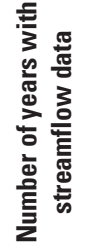 & 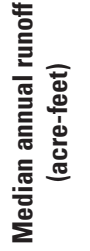 & 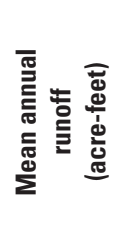 & 离 & 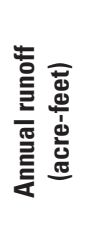 \\
\hline
\end{tabular}

\begin{tabular}{|c|c|c|c|c|c|c|c|c|c|c|c|}
\hline \multicolumn{12}{|c|}{ Missouri River Basin } \\
\hline 161 & 06036650 & $\begin{array}{l}\text { Jefferson River near Three } \\
\text { Forks, Montana }\end{array}$ & 9,532 & $2,208,000$ & 4 & $\begin{array}{c}1895,1897- \\
1898 \\
1900- \\
1905 \\
1939- \\
1969 \\
1979- \\
2011\end{array}$ & 72 & $1,473,000$ & $1,457,000$ & 1984 & $2,643,000$ \\
\hline 165 & 06041000 & $\begin{array}{l}\text { Madison River below Ennis } \\
\text { Lake near McAllister, } \\
\text { Montana }\end{array}$ & 2,186 & $1,470,000$ & 19 & 1939-2011 & 73 & $1,216,000$ & $1,271,000$ & 1997 & $1,832,000$ \\
\hline 167 & 06052500 & $\begin{array}{l}\text { Gallatin River at Logan, } \\
\text { Montana }\end{array}$ & 1,795 & $1,137,000$ & 6 & $\begin{array}{l}1894-1905 \\
\quad 1929- \\
2011\end{array}$ & 95 & 760,200 & 770,100 & 1997 & $1,209,000$ \\
\hline 168 & 06054500 & $\begin{array}{l}\text { Missouri River at Toston, } \\
\text { Montana }\end{array}$ & 14,669 & $5,010,000$ & 7 & $\begin{array}{c}1911-16 \\
1942- \\
2011\end{array}$ & 76 & $3,660,000$ & $3,705,000$ & 1997 & $5,604,000$ \\
\hline 177 & 06089000 & $\begin{array}{l}\text { Sun River near Vaughn, } \\
\quad \text { Montana }\end{array}$ & 1,849 & 818,100 & 6 & $1935-2011$ & 77 & 469,100 & 486,900 & 1943 & 948,400 \\
\hline 180 & 06090800 & $\begin{array}{l}\text { Missouri River at Fort Benton, } \\
\text { Montana }\end{array}$ & 24,749 & $8,833,000$ & 1 & $1891-2011$ & 121 & $5,459,000$ & $5,511,000$ & 1894 & $8,615,000$ \\
\hline 183 & 06101500 & $\begin{array}{l}\text { Marias River near Chester, } \\
\text { Montana }\end{array}$ & 4,927 & 832,600 & 11 & $\begin{array}{c}1946-47 \\
1956- \\
2011\end{array}$ & 58 & 540,400 & 579,200 & 1959 & $1,079,000$ \\
\hline 189 & 06115200 & $\begin{array}{l}\text { Missouri River near Landusky, } \\
\quad \text { Montana }\end{array}$ & 40,987 & $10,710,000$ & 2 & $1935-2011$ & 77 & $6,349,000$ & $6,510,000$ & 1975 & $11,080,000$ \\
\hline 199 & 06130500 & $\begin{array}{l}\text { Musselshell River at Mosby, } \\
\text { Montana }\end{array}$ & 7,846 & $1,419,000$ & 1 & $\begin{array}{c}1931-32 \\
1935- \\
2011\end{array}$ & 79 & 129,600 & 199,200 & 1978 & 789,100 \\
\hline 201 & 06132000 & $\begin{array}{l}\text { Missouri River below Fort } \\
\text { Peck Dam, Montana }\end{array}$ & 57,556 & $12,810,000$ & 1 & $\begin{array}{l}1935-2000 \\
\quad 2002- \\
2011\end{array}$ & 76 & $6,085,000$ & $6,393,000$ & 1975 & $10,860,000$ \\
\hline 210 & 06174500 & $\begin{array}{l}\text { Milk River at Nashua, Mon- } \\
\text { tana }\end{array}$ & 22,332 & $2,483,000$ & 1 & $1940-2011$ & 72 & 380,100 & 475,800 & 1952 & $1,716,000$ \\
\hline 213 & 06185500 & $\begin{array}{l}\text { Missouri River near Culbert- } \\
\text { son, Montana }\end{array}$ & 91,557 & $16,650,000$ & 1 & $\begin{array}{l}1942-1951 \\
1959- \\
2011\end{array}$ & 63 & $6,653,000$ & $7,220,000$ & 1975 & $12,020,000$ \\
\hline 218 & 06192500 & $\begin{array}{l}\text { Yellowstone River near Liv- } \\
\text { ingston, Montana }\end{array}$ & 3,551 & $4,134,000$ & 2 & $\begin{array}{c}1898-1905 \\
1929- \\
1932 \\
1938- \\
2011\end{array}$ & 86 & $2,632,000$ & $2,713,000$ & 1997 & $4,431,000$ \\
\hline 222 & 06207500 & $\begin{array}{l}\text { Clarks Fork Yellowstone River } \\
\text { near Belfry, Montana }\end{array}$ & 1,154 & $1,014,000$ & 4 & $1922-2011$ & 90 & 648,300 & 676,200 & 1997 & $1,079,000$ \\
\hline 227 & 06214500 & $\begin{array}{l}\text { Yellowstone River at Billings, } \\
\quad \text { Montana }\end{array}$ & 11,408 & $8,181,000$ & 2 & 1929-2011 & 83 & $5,090,000$ & $5,027,000$ & 1997 & $8,760,000$ \\
\hline 239 & 06289000 & $\begin{array}{l}\text { Little Bighorn River at State } \\
\text { line near Wyola, Montana }\end{array}$ & 182 & 173,000 & 2 & $1940-2011$ & 72 & 103,900 & 108,000 & 1975 & 183,200 \\
\hline
\end{tabular}


Estimated

AEP for

observed

2011

annual

runoff

(percent)
Expected annual runoff in acre-feet for selected annual exceedance probabilities (AEP) based on Water Resources Council Bulletin 17B methods

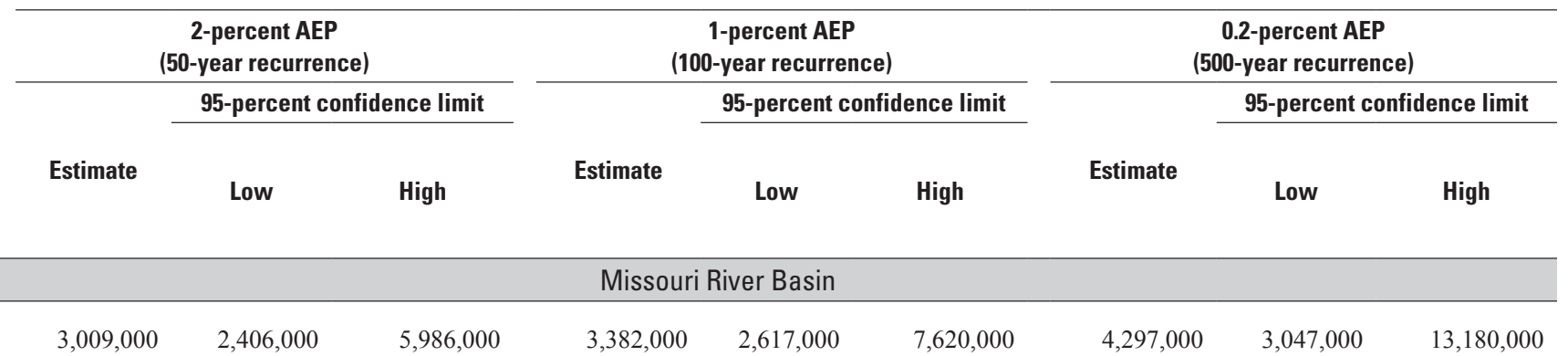

\begin{tabular}{|c|c|c|c|c|c|c|c|c|c|}
\hline$>10$ & $1,783,000$ & $1,669,000$ & $2,009,000$ & $1,863,000$ & $1,723,000$ & $2,152,000$ & $2,032,000$ & $1,821,000$ & $2,490,000$ \\
\hline 4 to 10 & $1,260,000$ & $1,154,000$ & $1,445,000$ & $1,340,000$ & $1,205,000$ & $1,576,000$ & $1,508,000$ & $1,292,000$ & $1,890,000$ \\
\hline 4 to 10 & $5,766,000$ & $5,284,000$ & $6,592,000$ & $6,075,000$ & $5,458,000$ & $7,111,000$ & $6,711,000$ & $5,718,000$ & $8,332,000$ \\
\hline 4 to 10 & $1,003,000$ & 863,000 & $1,254,000$ & $1,095,000$ & 909,800 & $1,424,000$ & $1,295,000$ & 978,700 & $1,858,000$ \\
\hline 2 to 4 & $8,964,000$ & $8,284,000$ & $10,030,000$ & $9,506,000$ & $8,635,000$ & $10,860,000$ & $10,640,000$ & $9,235,000$ & $12,810,000$ \\
\hline$>10$ & 980,000 & 501,800 & $1,167,000$ & $1,006,000$ & 466,100 & $1,263,000$ & $1,042,000$ & 546,900 & $1,531,000$ \\
\hline 2 to 4 & $11,000,000$ & $9,925,000$ & $13,020,000$ & $11,750,000$ & $10,380,000$ & $14,350,000$ & $13,340,000$ & $11,140,000$ & $17,590,000$ \\
\hline$<0.2$ & 801,400 & 524,200 & $1,244,000$ & 951,800 & 538,900 & $1,595,000$ & $1,295,000$ & 533,200 & $2,679,000$ \\
\hline 0.2 to 1 & $11,780,000$ & $10,430,000$ & $14,570,000$ & $12,780,000$ & $11,060,000$ & $16,490,000$ & $14,990,000$ & $12,200,000$ & $21,430,000$ \\
\hline 0.2 to 1 & $1,848,000$ & $1,344,000$ & $3,256,000$ & $2,274,000$ & $1,561,000$ & $4,492,000$ & $3,422,000$ & $2,006,000$ & $8,877,000$ \\
\hline 0.2 to 1 & $13,530,000$ & $11,740,000$ & $18,600,000$ & $14,890,000$ & $12,630,000$ & $22,270,000$ & $18,110,000$ & $14,490,000$ & $33,300,000$ \\
\hline 1 to 2 & $4,051,000$ & $3,757,000$ & $4,747,000$ & $4,282,000$ & $3,921,000$ & $5,221,000$ & $4,789,000$ & $4,239,000$ & $6,427,000$ \\
\hline
\end{tabular}

\begin{tabular}{rrrrrrrrrr}
\hline 2 to 4 & $1,069,000$ & 979,400 & $1,262,000$ & $1,143,000$ & $1,033,000$ & $1,408,000$ & $1,313,000$ & $1,142,000$ & $1,788,000$ \\
\hline 1 to 2 & $8,104,000$ & $7,399,000$ & $9,536,000$ & $8,647,000$ & $7,773,000$ & $10,540,000$ & $9,847,000$ & $8,484,000$ & $13,070,000$ \\
\hline 2 to 4 & 174,300 & 158,400 & 205,500 & 185,200 & 165,300 & 225,500 & 208,700 & 177,000 & 274,100
\end{tabular}


Table 2. Annual runoff data and results of flood-probability analyses for selected streamgages in the Central United States affected by 2011 flooding.-Continued

[Stations used in the trend analysis are italicized. $\mathrm{mi}^{2}$, square miles; AEP, annual exceedance probability; <, less than; >, greater than]

\begin{tabular}{|c|c|c|c|c|c|c|c|c|c|c|c|}
\hline \multirow{2}{*}{ 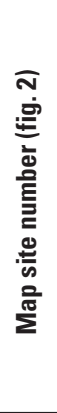 } & \multirow[b]{2}{*}{$\begin{array}{l}\text { Station } \\
\text { number }\end{array}$} & \multirow[b]{2}{*}{ Station name } & \multirow{2}{*}{ 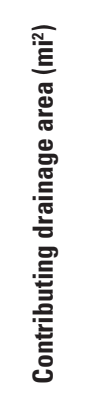 } & \multicolumn{2}{|c|}{ Water year 2011} & \multicolumn{4}{|c|}{ Period of record } & \multicolumn{2}{|c|}{$\begin{array}{c}\text { Previous maximum } \\
\text { annual runoff based } \\
\text { on data through } \\
\text { water year } 2010^{\mathrm{a}}\end{array}$} \\
\hline & & & & 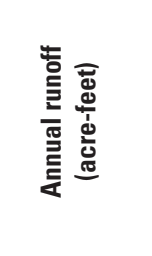 & 訔 & 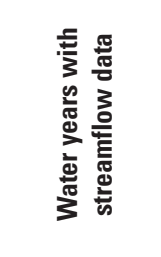 & 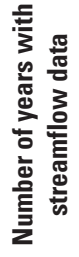 & 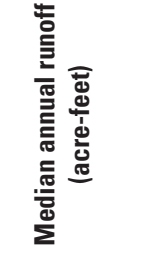 & 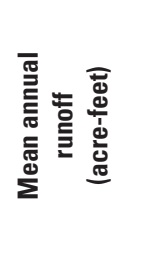 & 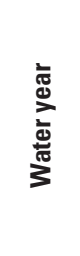 & 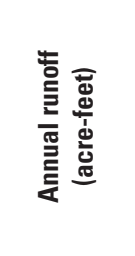 \\
\hline \multicolumn{12}{|c|}{ Missouri River Basin-Continued } \\
\hline 255 & 06308500 & $\begin{array}{l}\text { Tongue River at Miles City, } \\
\text { Montana }\end{array}$ & 5,397 & 711,700 & 2 & $\begin{array}{c}1939-41 \\
1947- \\
2011\end{array}$ & 68 & 291,400 & 296,300 & 1978 & 713,800 \\
\hline 256 & 06309000 & $\begin{array}{l}\text { Yellowstone River at Miles } \\
\text { City, Montana }\end{array}$ & 48,253 & $14,330,000$ & 1 & $\begin{array}{l}1923,1929 \\
2011\end{array}$ & 84 & $8,507,000$ & $8,177,000$ & 1997 & $12,670,000$ \\
\hline 258 & 06326500 & $\begin{array}{l}\text { Powder River near Locate, } \\
\quad \text { Montana }\end{array}$ & 13,068 & 948,400 & 3 & $1939-2011$ & 73 & 396,000 & 412,900 & 1944 & $1,180,000$ \\
\hline 260 & 06329500 & $\begin{array}{l}\text { Yellowstone River near Sidney, } \\
\text { Montana }\end{array}$ & 68,392 & $16,000,000$ & 1 & $\begin{array}{c}1911-31 \\
1934- \\
2011\end{array}$ & 99 & $9,339,000$ & $9,027,000$ & 1924 & $15,420,000$ \\
\hline 269 & 06337000 & $\begin{array}{l}\text { Little Missouri River near Wat- } \\
\quad \text { ford City, North Dakota }\end{array}$ & 8,310 & $1,477,000$ & 1 & $1935-2011$ & 77 & 329,400 & 400,900 & 1971 & $1,187,000$ \\
\hline 270 & 06340500 & $\begin{array}{l}\text { Knife River at Hazen, North } \\
\text { Dakota }\end{array}$ & 2,240 & 345,300 & 2 & $\begin{array}{c}1930-33 \\
1938- \\
2011\end{array}$ & 78 & 99,180 & 122,100 & 2009 & 373,600 \\
\hline 271 & 06342500 & $\begin{array}{l}\text { Missouri River at Bismarck, } \\
\text { North Dakota }\end{array}$ & 186,400 & $38,520,000$ & 1 & 1929-2011 & 83 & $15,350,000$ & $16,060,000$ & 1975 & $25,770,000$ \\
\hline 272 & 06349000 & $\begin{array}{l}\text { Heart River at Mandan, North } \\
\quad \text { Dakota }\end{array}$ & 3,310 & 676,200 & 2 & $\begin{array}{c}1929-32 \\
1938- \\
2011\end{array}$ & 78 & 140,100 & 195,200 & 2009 & 687,100 \\
\hline 273 & 06354000 & $\begin{array}{l}\text { Cannonball River at Breien, } \\
\quad \text { North Dakota }\end{array}$ & 4,100 & 572,700 & 5 & 1935-2011 & 77 & 124,500 & 181,000 & 2009 & 738,500 \\
\hline 274 & 06357800 & $\begin{array}{l}\text { Grand River at Little Eagle, } \\
\text { South Dakota }\end{array}$ & 5,322 & 573,400 & 3 & 1959-2011 & 53 & 128,100 & 192,900 & 2009 & 818,100 \\
\hline 275 & 06359500 & $\begin{array}{l}\text { Moreau River near Faith, } \\
\text { South Dakota }\end{array}$ & 2,596 & 351,100 & 4 & $1944-2011$ & 68 & 68,780 & 107,000 & 2009 & 411,900 \\
\hline 285 & 06438500 & $\begin{array}{l}\text { Cheyenne River near Plain- } \\
\text { view, South Dakota }\end{array}$ & 21,414 & $1,578,000$ & 3 & $\begin{array}{l}\text { 1951-1981, } \\
1995- \\
2011\end{array}$ & 48 & 468,800 & 573,200 & 1997 & $1,752,000$ \\
\hline 287 & 06452000 & $\begin{array}{l}\text { White River near Oacoma, } \\
\quad \text { South Dakota }\end{array}$ & 9,859 & $1,122,000$ & 3 & $1929-2011$ & 83 & 372,800 & 425,700 & 1942 & $1,252,000$ \\
\hline 289 & 06465500 & $\begin{array}{l}\text { Niobrara River near Verdel, } \\
\quad \text { Nebraska }\end{array}$ & 11,580 & $1,853,000$ & 2 & $\begin{array}{l}1939,1959 \\
2011\end{array}$ & 54 & $1,166,000$ & $1,269,000$ & 2010 & $1,976,000$ \\
\hline 294 & 06470500 & $\begin{array}{l}\text { James River at LaMoure, } \\
\quad \text { North Dakota }\end{array}$ & 1,790 & $1,050,000$ & 1 & $1951-2011$ & 61 & 78,190 & 151,600 & 2009 & 810,900 \\
\hline 305 & 06478500 & $\begin{array}{l}\text { James River near Scotland, } \\
\quad \text { South Dakota }\end{array}$ & 18,601 & $4,199,000$ & 2 & 1929-2011 & 83 & 254,800 & 558,100 & 2010 & $4,214,000$ \\
\hline 310 & 06485500 & Big Sioux River at Akron, Iowa & 6,996 & $4,214,000$ & 2 & $1929-2011$ & 83 & 760,200 & 995,300 & 1993 & $4,539,000$ \\
\hline 311 & 06486000 & $\begin{array}{l}\text { Missouri River at Sioux City, } \\
\text { Iowa }\end{array}$ & 314,600 & $57,270,000$ & 1 & $\begin{array}{c}1929-31 \\
1939 \\
2011\end{array}$ & 76 & $20,600,000$ & $21,720,000$ & 1997 & $40,470,000$ \\
\hline 312 & 06600500 & Floyd River at James, Iowa & 886 & 607,400 & 5 & $1936-2011$ & 76 & 150,900 & 198,300 & 1983 & 693,600 \\
\hline 314 & 06607500 & $\begin{array}{l}\text { Little Sioux River near Turin, } \\
\quad \text { Iowa }\end{array}$ & 3,526 & $2,382,000$ & 5 & $\begin{array}{c}1943-57 \\
1959- \\
2011\end{array}$ & 68 & 894,100 & 961,800 & 1993 & $3,808,000$ \\
\hline
\end{tabular}




\begin{tabular}{|c|c|c|c|c|c|c|c|c|c|}
\hline \multirow{3}{*}{$\begin{array}{l}\text { Estimated } \\
\text { AEP for } \\
\text { observed } \\
2011 \\
\text { annual } \\
\text { runoff } \\
\text { (percent) }\end{array}$} & \multicolumn{3}{|c|}{$\begin{array}{c}\text { 2-percent AEP } \\
\text { (50-year recurrence) }\end{array}$} & \multicolumn{3}{|c|}{$\begin{array}{c}\text { 1-percent AEP } \\
\text { (100-year recurrence) }\end{array}$} & \multicolumn{3}{|c|}{$\begin{array}{c}\text { 0.2-percent AEP } \\
\text { (500-year recurrence) }\end{array}$} \\
\hline & \multirow[b]{2}{*}{ Estimate } & \multicolumn{2}{|c|}{ 95-percent confidence limit } & \multirow[b]{2}{*}{ Estimate } & \multicolumn{2}{|c|}{ 95-percent confidence limit } & \multirow[b]{2}{*}{ Estimate } & \multicolumn{2}{|c|}{ 95-percent confidence limit } \\
\hline & & Low & High & & Low & High & & Low & High \\
\hline \multicolumn{10}{|c|}{ Missouri River Basin-Continued } \\
\hline$<0.2$ & 600,000 & 329,000 & 729,100 & 632,800 & 304,500 & 805,300 & 687,600 & 321,300 & 999,500 \\
\hline 0.2 to 1 & $13,030,000$ & $11,940,000$ & $14,950,000$ & $13,790,000$ & $12,400,000$ & $16,220,000$ & $15,390,000$ & $13,160,000$ & $19,260,000$ \\
\hline 1 to 2 & 933,000 & 687,400 & $1,165,000$ & $1,009,000$ & 664,200 & $1,314,000$ & $1,154,000$ & 628,700 & $1,694,000$ \\
\hline 1 to 2 & $15,250,000$ & $13,870,000$ & $17,430,000$ & $16,220,000$ & $14,430,000$ & $18,990,000$ & $18,230,000$ & $15,300,000$ & $22,690,000$ \\
\hline 0.2 to 1 & $1,213,000$ & 896,900 & $1,690,000$ & $1,380,000$ & 923,500 & $2,034,000$ & $1,741,000$ & 927,600 & $2,998,000$ \\
\hline 2 to 4 & 389,600 & 298,500 & 569,300 & 453,000 & 324,700 & 706,300 & 601,700 & 361,700 & $1,108,000$ \\
\hline 0.2 to 1 & $28,660,000$ & $25,340,000$ & $37,940,000$ & $31,540,000$ & $27,310,000$ & $45,450,000$ & $38,610,000$ & $31,700,000$ & $69,120,000$ \\
\hline 2 to 4 & 808,700 & 580,000 & $1,345,000$ & 989,200 & 658,700 & $1,801,000$ & $1,454,000$ & 792,200 & $3,315,000$ \\
\hline 4 to 10 & 830,200 & 579,000 & $1,537,000$ & $1,053,000$ & 686,500 & $2,202,000$ & $1,679,000$ & 910,500 & $4,699,000$ \\
\hline 4 to 10 & 896,100 & 583,300 & $2,545,000$ & $1,173,000$ & 714,000 & $4,422,000$ & $2,040,000$ & $1,040,000$ & $15,220,000$ \\
\hline 4 to 10 & 517,500 & 348,600 & $1,032,000$ & 661,100 & 413,000 & $1,505,000$ & $1,067,000$ & 542,000 & $3,336,000$ \\
\hline 4 to 10 & $2,109,000$ & $1,477,000$ & $5,527,000$ & $2,595,000$ & $1,716,000$ & $8,805,000$ & $3,941,000$ & $2,233,000$ & $24,690,000$ \\
\hline 2 to 4 & $1,212,000$ & 969,300 & $1,865,000$ & $1,430,000$ & $1,103,000$ & $2,447,000$ & $2,007,000$ & $1,404,000$ & $4,427,000$ \\
\hline 2 to 4 & $1,989,000$ & $1,770,000$ & $2,886,000$ & $2,155,000$ & $1,878,000$ & $3,402,000$ & $2,563,000$ & $2,122,000$ & $4,968,000$ \\
\hline 1 to 2 & 958,600 & 564,400 & $3,170,000$ & $1,362,000$ & 736,900 & $6,178,000$ & $2,800,000$ & $1,214,000$ & $27,270,000$ \\
\hline 1 to 2 & $3,616,000$ & $2,248,000$ & $8,126,000$ & $5,020,000$ & $2,861,000$ & $13,300,000$ & $9,587,000$ & $4,314,000$ & $37,630,000$ \\
\hline 1 to 2 & $4,119,000$ & $2,990,000$ & $7,285,000$ & $5,172,000$ & $3,552,000$ & $10,350,000$ & $8,153,000$ & $4,823,000$ & $21,910,000$ \\
\hline$<0.2$ & $40,560,000$ & $35,250,000$ & $57,780,000$ & $45,130,000$ & $38,280,000$ & $72,210,000$ & $56,640,000$ & $45,200,000$ & $113,900,000$ \\
\hline 2 to 4 & 687,700 & 494,300 & $1,020,000$ & 801,600 & 520,100 & $1,270,000$ & $1,061,000$ & 537,900 & $2,010,000$ \\
\hline 2 to 4 & $2,386,000$ & 396,600 & $7,534,000$ & $2,407,000$ & 632,200 & $11,950,000$ & $2,420,000$ & 843,300 & $32,990,000$ \\
\hline
\end{tabular}


Table 2. Annual runoff data and results of flood-probability analyses for selected streamgages in the Central United States affected by 2011 flooding.-Continued

[Stations used in the trend analysis are italicized. square miles; AEP, annual exceedance probability; <, less than; >, greater than]

\begin{tabular}{|c|c|c|c|c|c|c|c|c|c|c|c|}
\hline \multirow{2}{*}{ 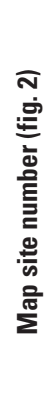 } & \multirow{2}{*}{$\begin{array}{l}\text { Station } \\
\text { number }\end{array}$} & \multirow[b]{2}{*}{ Station name } & \multirow{2}{*}{ 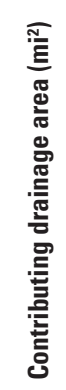 } & \multicolumn{2}{|c|}{ Water year 2011} & \multicolumn{4}{|c|}{ Period of record } & \multicolumn{2}{|c|}{$\begin{array}{l}\text { Previous maximum } \\
\text { annual runoff based } \\
\text { on data through } \\
\text { water year } 2010^{\text {a }}\end{array}$} \\
\hline & & & & 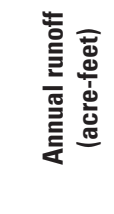 & 套 & 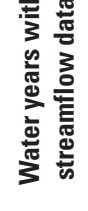 & 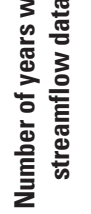 & 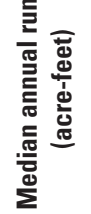 & 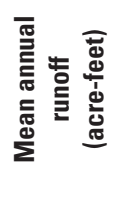 & 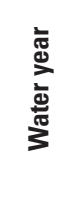 & 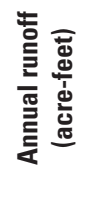 \\
\hline
\end{tabular}

\begin{tabular}{|c|c|c|c|c|c|c|c|c|c|c|c|}
\hline \multicolumn{12}{|c|}{ Missouri River Basin-Continued } \\
\hline 321 & 06674500 & $\begin{array}{l}\text { North Platte River at Wyoming- } \\
\quad \text { Nebraska State Line }\end{array}$ & 22,218 & $2,172,000$ & 1 & $1930-2011$ & 82 & 444,500 & 568,400 & 1984 & $2,078,000$ \\
\hline 325 & 06774000 & $\begin{array}{l}\text { Platte River near Ducan, } \\
\quad \text { Nebraska }\end{array}$ & 54,630 & $3,258,000$ & 3 & $1930-2011$ & 82 & 991,800 & $1,255,000$ & 1984 & $4,822,000$ \\
\hline 326 & 06793000 & $\begin{array}{l}\text { Loup River near Genoa, } \\
\quad \text { Nebraska }\end{array}$ & 5,620 & 718,900 & 19 & $\begin{array}{c}1930-31 \\
1944- \\
2011\end{array}$ & 70 & 494,500 & 583,900 & 1930 & $1,976,000$ \\
\hline 327 & 06796000 & $\begin{array}{l}\text { Platte River at North Bend, } \\
\quad \text { Nebraska }\end{array}$ & 57,800 & $4,959,000$ & 7 & $1950-2011$ & 62 & $3,077,000$ & $3,308,000$ & 1984 & $7,312,000$ \\
\hline 329 & 06810000 & $\begin{array}{l}\text { Nishnabotna River above } \\
\text { Hamburg, Iowa }\end{array}$ & 2,806 & $1,455,000$ & 13 & $\begin{array}{l}1923,1929- \\
2011\end{array}$ & 84 & 810,900 & 965,800 & 1993 & $3,663,000$ \\
\hline 330 & 06813500 & $\begin{array}{l}\text { Missouri River at Rulo, } \\
\quad \text { Nebraska }\end{array}$ & 414,900 & $72,400,000$ & 1 & $1950-2011$ & 62 & $29,320,000$ & $31,340,000$ & 1997 & $52,050,000$ \\
\hline 331 & 06815000 & $\begin{array}{l}\text { Big Nemaha River at Falls } \\
\text { City, Nebraska }\end{array}$ & 1,339 & 207,800 & 47 & $1945-2011$ & 67 & 353,300 & 437,600 & 1993 & $1,853,000$ \\
\hline 333 & 06818000 & $\begin{array}{l}\text { Missouri River at St. Joseph, } \\
\quad \text { Missouri }\end{array}$ & 426,500 & $75,290,000$ & 1 & $1929-2011$ & 83 & $29,030,000$ & $31,540,000$ & 1997 & $55,020,000$ \\
\hline 338 & 06892350 & $\begin{array}{l}\text { Kansas River at De Soto, } \\
\quad \text { Kansas }\end{array}$ & 59,756 & $4,047,000$ & 55 & $1918-2011$ & 94 & $4,554,000$ & $5,325,000$ & 1993 & $22,150,000$ \\
\hline 340 & 06902000 & $\begin{array}{l}\text { Grand River near Sumner, } \\
\quad \text { Missouri }\end{array}$ & 6,880 & $2,773,000$ & 46 & $1925-2011$ & 87 & $2,795,000$ & $3,120,000$ & 1993 & $12,600,000$ \\
\hline 341 & 06905500 & $\begin{array}{l}\text { Chariton River near Prairie } \\
\text { Hill, Missouri }\end{array}$ & 1,870 & $1,318,000$ & 21 & $1930-2011$ & 82 & 850,700 & 959,500 & 2010 & $3,301,000$ \\
\hline 342 & 06926000 & $\begin{array}{l}\text { Osage River near Bagnell, } \\
\text { Missouri }\end{array}$ & 14,000 & $6,914,000$ & 46 & $1926-2011$ & 86 & $7,385,000$ & $7,653,000$ & 1927 & $17,810,000$ \\
\hline 346 & 06934500 & $\begin{array}{l}\text { Missouri River at Hermann, } \\
\text { Missouri }\end{array}$ & 522,500 & $100,600,000$ & 5 & $1929-2011$ & 83 & $57,270,000$ & $59,430,000$ & 1993 & $131,800,000$ \\
\hline \multicolumn{12}{|c|}{ Ohio River Basin } \\
\hline 347 & 03016000 & $\begin{array}{l}\text { Allegheny River at West } \\
\text { Hickory, Pennsylvania }\end{array}$ & 3,660 & $7,095,000$ & 2 & $\begin{array}{c}1942-2005 \\
2008- \\
2011\end{array}$ & 68 & $4,901,000$ & $4,880,000$ & 2004 & $7,146,000$ \\
\hline 348 & 03031500 & $\begin{array}{l}\text { Allegheny River at Parker, } \\
\text { Pennsylvania }\end{array}$ & 7,671 & $13,830,000$ & 3 & 1933-2011 & 79 & $9,991,000$ & $10,050,000$ & 2004 & $15,490,000$ \\
\hline 349 & 03044000 & $\begin{array}{l}\text { Connemaugh River at Tun- } \\
\text { nelton, Pennsylvania }\end{array}$ & 1,358 & $2,150,000$ & 5 & $\begin{array}{c}\text { 1940-1991 } \\
2010- \\
2011\end{array}$ & 54 & $1,658,000$ & $1,730,000$ & 1951 & $2,519,000$ \\
\hline 350 & 03049500 & $\begin{array}{l}\text { Allegheny River at Natrona, } \\
\text { Pennsylvania }\end{array}$ & 11,410 & $19,400,000$ & 3 & 1939-2011 & 73 & $14,260,000$ & $14,340,000$ & 2004 & $21,790,000$ \\
\hline 351 & 03069500 & $\begin{array}{l}\text { Cheat River near Parsons, } \\
\text { West Virginia }\end{array}$ & 722 & $1,477,000$ & 19 & 1914-2011 & 98 & $1,256,000$ & $1,261,000$ & 1996 & $2,266,000$ \\
\hline 352 & 03075070 & $\begin{array}{l}\text { Monongahela River at Eliza- } \\
\text { beth, Pennsylvania }\end{array}$ & 5,340 & $7,529,000$ & 22 & 1934-2011 & 78 & $6,679,000$ & $6,693,000$ & 1996 & $10,430,000$ \\
\hline 353 & 03086000 & $\begin{array}{l}\text { Ohio River at Sewickley, Penn- } \\
\text { sylvania }\end{array}$ & 19,500 & $30,990,000$ & 7 & 1934-2011 & 78 & $23,750,000$ & $24,390,000$ & 2004 & $37,210,000$ \\
\hline 356 & 03129000 & $\begin{array}{l}\text { Tuscarawas River at Newcom- } \\
\text { erstown, Ohio }\end{array}$ & 2,443 & $2,780,000$ & 6 & $1922-2011$ & 90 & $1,843,000$ & $1,900,000$ & 2004 & $3,779,000$ \\
\hline
\end{tabular}


Expected annual runoff in acre-feet for selected annual exceedance probabilities (AEP) based on Water Resources Council Bulletin 17B methods

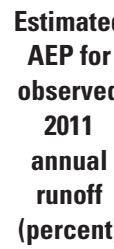

Low High Estimate

\begin{tabular}{cc}
\hline $\begin{array}{c}\text { 2-percent AEP } \\
(50-y e a r \text { recurrence })\end{array}$ \\
\cline { 2 - 2 } Estimate
\end{tabular}

1-percent AEP

(100-year recurrence)

95-percent confidence limit

(percent)
Estimate

Low

High

0.2-percent AEP

(500-year recurrence)

95-percent confidence limit

Estimate

Low

High

\begin{tabular}{|c|c|c|c|c|c|c|c|c|c|}
\hline \multicolumn{10}{|c|}{ Missouri River Basin-Continued } \\
\hline 1 to 2 & $1,780,000$ & $1,309,000$ & $4,782,000$ & $2,288,000$ & $1,579,000$ & $8,001,000$ & $4,010,000$ & $2,358,000$ & $26,950,000$ \\
\hline 2 to 4 & $3,379,000$ & $2,708,000$ & $4,563,000$ & $3,821,000$ & $2,889,000$ & $5,433,000$ & $4,810,000$ & $3,127,000$ & $7,814,000$ \\
\hline$>10$ & $1,572,000$ & $1,254,000$ & $2,536,000$ & $1,842,000$ & $1,416,000$ & $3,359,000$ & $2,547,000$ & $1,780,000$ & $6,261,000$ \\
\hline 4 to 10 & $6,569,000$ & $5,575,000$ & $10,140,000$ & $7,363,000$ & $6,082,000$ & $13,060,000$ & $9,364,000$ & $7,222,000$ & $21,120,000$ \\
\hline$>10$ & $2,978,000$ & $2,346,000$ & $4,347,000$ & $3,482,000$ & $2,613,000$ & $5,456,000$ & $4,714,000$ & $3,098,000$ & $8,775,000$ \\
\hline 0.2 to 1 & $58,950,000$ & $50,290,000$ & $96,130,000$ & $66,060,000$ & $54,800,000$ & $120,200,000$ & $84,510,000$ & $65,330,000$ & $201,300,000$ \\
\hline$>10$ & $1,554,000$ & $1,151,000$ & $2,712,000$ & $1,882,000$ & $1,323,000$ & $3,690,000$ & $2,754,000$ & $1,676,000$ & $7,100,000$ \\
\hline 0.2 to 1 & $59,440,000$ & $52,190,000$ & $77,740,000$ & $65,620,000$ & $56,390,000$ & $92,200,000$ & $80,560,000$ & $65,480,000$ & $135,100,000$ \\
\hline$>10$ & $15,940,000$ & $12,760,000$ & $25,350,000$ & $18,870,000$ & $14,550,000$ & $33,710,000$ & $26,520,000$ & $18,470,000$ & $62,730,000$ \\
\hline$>10$ & $9,304,000$ & $6,826,000$ & $12,520,000$ & $10,500,000$ & $6,919,000$ & $14,910,000$ & $13,000,000$ & $6,837,000$ & $21,400,000$ \\
\hline$>10$ & $2,751,000$ & $2,009,000$ & $3,684,000$ & $3,084,000$ & $2,015,000$ & $4,353,000$ & $3,773,000$ & $1,962,000$ & $6,162,000$ \\
\hline$>10$ & $18,950,000$ & $13,350,000$ & $23,880,000$ & $20,640,000$ & $12,870,000$ & $27,250,000$ & $23,840,000$ & $12,310,000$ & $35,870,000$ \\
\hline 4 to 10 & $118,400,000$ & $103,500,000$ & $147,800,000$ & $129,600,000$ & $110,400,000$ & $168,900,000$ & $154,700,000$ & $122,800,000$ & $223,500,000$ \\
\hline
\end{tabular}

\begin{tabular}{|c|c|c|c|c|c|c|c|c|c|}
\hline \multicolumn{10}{|c|}{ Ohio River Basin } \\
\hline 1 to 2 & $7,094,000$ & $6,575,000$ & $8,139,000$ & $7,441,000$ & $6,803,000$ & $8,776,000$ & $8,176,000$ & $7,197,000$ & $10,290,000$ \\
\hline 2 to 4 & $14,460,000$ & $13,500,000$ & $16,160,000$ & $15,120,000$ & $13,910,000$ & $17,250,000$ & $16,470,000$ & $14,580,000$ & $19,760,000$ \\
\hline$>10$ & $2,461,000$ & $2,275,000$ & $2,829,000$ & $2,567,000$ & $2,335,000$ & $3,023,000$ & $2,784,000$ & $2,421,000$ & $3,480,000$ \\
\hline 2 to 4 & $20,440,000$ & $19,070,000$ & $23,220,000$ & $21,410,000$ & $19,730,000$ & $24,990,000$ & $23,490,000$ & $20,950,000$ & $29,230,000$ \\
\hline$>10$ & $1,880,000$ & $1,749,000$ & $2,146,000$ & $1,991,000$ & $1,830,000$ & $2,346,000$ & $2,237,000$ & $1,992,000$ & $2,848,000$ \\
\hline$>10$ & $10,150,000$ & $9,358,000$ & $11,730,000$ & $10,720,000$ & $9,749,000$ & $12,780,000$ & $11,960,000$ & $10,470,000$ & $15,330,000$ \\
\hline 4 to 10 & $35,130,000$ & $32,740,000$ & $41,170,000$ & $36,920,000$ & $33,990,000$ & $45,050,000$ & $40,780,000$ & $36,370,000$ & $54,860,000$ \\
\hline 4 to 10 & $3,066,000$ & $2,771,000$ & $3,447,000$ & $3,223,000$ & $2,823,000$ & $3,697,000$ & $3,529,000$ & $2,869,000$ & $4,274,000$ \\
\hline
\end{tabular}




\section{Annual Exceedance Probabilities and Trends for the Central United States during the 2011 Floods}

Table 2. Annual runoff data and results of flood-probability analyses for selected streamgages in the Central United States affected by 2011 flooding.-Continued

[Stations used in the trend analysis are italicized. $\mathrm{mi}^{2}$, square miles; AEP, annual exceedance probability; <, less than; >, greater than]

\begin{tabular}{|c|c|c|c|c|c|c|c|c|c|c|c|}
\hline \multirow{2}{*}{ 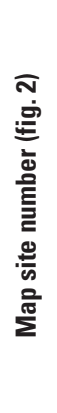 } & \multirow[b]{2}{*}{$\begin{array}{l}\text { Station } \\
\text { number }\end{array}$} & \multirow[b]{2}{*}{ Station name } & \multirow{2}{*}{ 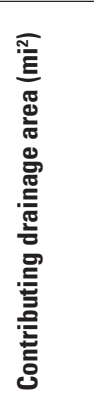 } & \multicolumn{2}{|c|}{ Water year 2011} & \multicolumn{4}{|c|}{ Period of record } & \multicolumn{2}{|c|}{$\begin{array}{l}\text { Previous maximum } \\
\text { annual runoff based } \\
\text { on data through } \\
\text { water year } 2010^{\mathrm{a}}\end{array}$} \\
\hline & & & & 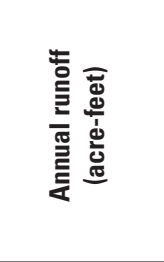 & 荡 & 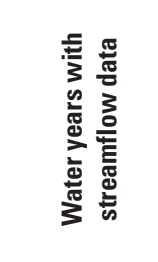 & 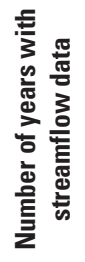 & 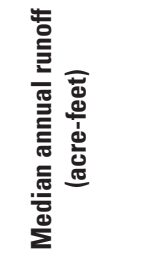 & 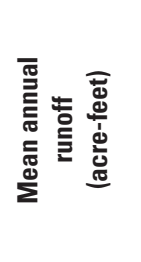 & 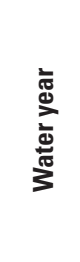 & 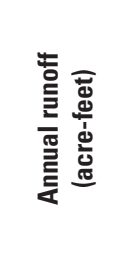 \\
\hline \multicolumn{12}{|c|}{ Ohio River Basin-Continued } \\
\hline 358 & 03140500 & $\begin{array}{l}\text { Muskingum River near Co- } \\
\text { shocton, Ohio }\end{array}$ & 4,859 & $5,408,000$ & 4 & $1937-2011$ & 75 & $3,649,000$ & $3,739,000$ & 2004 & $6,907,000$ \\
\hline 359 & 03150000 & $\begin{array}{l}\text { Muskingum River at McCon- } \\
\text { nelsville, Ohio }\end{array}$ & 7,422 & $7,457,000$ & 12 & $\begin{array}{c}1922-92 \\
2002- \\
2011\end{array}$ & 81 & $5,495,000$ & $5,668,000$ & 2004 & $9,991,000$ \\
\hline 360 & 03155000 & $\begin{array}{l}\text { Little Kanawha River at Pales- } \\
\text { tine, West Virginia }\end{array}$ & 1,516 & $1,549,000$ & 35 & 1940-2011 & 72 & $1,524,000$ & $1,556,000$ & 1994 & $2,628,000$ \\
\hline 361 & 03159500 & Hocking River at Athens, Ohio & 943 & $1,071,000$ & 11 & $1916-2011$ & 96 & 722,200 & 746,300 & 2004 & $1,318,000$ \\
\hline 362 & 03176500 & $\begin{array}{l}\text { New River at Glen Lyn, } \\
\quad \text { Virginia }\end{array}$ & 3,783 & $3,497,000$ & 43 & $1928-2011$ & 84 & $3,500,000$ & $3,599,000$ & 2003 & $5,423,000$ \\
\hline 363 & 03184000 & $\begin{array}{l}\text { Greenbrier River at Hilldale, } \\
\text { West Virginia }\end{array}$ & 1,619 & $1,578,000$ & 39 & $1937-2011$ & 75 & $1,651,000$ & $1,656,000$ & 2003 & $3,157,000$ \\
\hline 364 & 03192000 & $\begin{array}{l}\text { Gauley River above Belva, } \\
\text { West Virginia }\end{array}$ & 1,317 & $2,020,000$ & 41 & $1930-2011$ & 82 & $1,998,000$ & $2,006,000$ & 2003 & $2,932,000$ \\
\hline 365 & 03193000 & $\begin{array}{l}\text { Kanawha River at Kanawha } \\
\text { Falls, West Virginia }\end{array}$ & 8,371 & $8,253,000$ & 81 & $1878-2011$ & 134 & $8,977,000$ & $9,071,000$ & 1901 & $15,350,000$ \\
\hline 366 & 03197000 & $\begin{array}{l}\text { Elk River at Queen Shoals, } \\
\text { West Virginia }\end{array}$ & 1,145 & $1,542,000$ & 37 & 1929-2011 & 83 & $1,499,000$ & $1,493,000$ & 1994 & $2,237,000$ \\
\hline 367 & 03198000 & $\begin{array}{l}\text { Kanawha River at Charleston, } \\
\text { West Virginia }\end{array}$ & 10,448 & $10,640,000$ & 42 & $1941-2011$ & 71 & $11,080,000$ & $11,040,000$ & 2004 & $16,510,000$ \\
\hline 368 & 03212500 & $\begin{array}{l}\text { Levisa Fork at Paintsville, } \\
\text { Kentucky }\end{array}$ & 2,144 & $2,179,000$ & 19 & $\begin{array}{l}1916,1929- \\
2011\end{array}$ & 84 & $1,774,000$ & $1,786,000$ & 1994 & $3,062,000$ \\
\hline 369 & 03213700 & $\begin{array}{l}\text { Tug Fork at Williamson, West } \\
\text { Virginia }\end{array}$ & 936 & 724,000 & 26 & 1968-2011 & 44 & 818,100 & 810,200 & 1979 & $1,252,000$ \\
\hline 370 & 03214500 & $\begin{array}{l}\text { Tug Fork at Kermit, West } \\
\text { Virginia }\end{array}$ & 1,280 & $1,021,000$ & 18 & $\begin{array}{l}\text { 1916-17, } \\
1930-34 \\
1986- \\
2011\end{array}$ & 33 & $1,057,000$ & $1,054,000$ & 1994 & $1,651,000$ \\
\hline 371 & 03227500 & $\begin{array}{l}\text { Scioto River at Columbus, } \\
\text { Ohio }\end{array}$ & 1,629 & $1,824,000$ & 2 & $\begin{array}{c}1921-90 \\
1992- \\
2011\end{array}$ & 90 & $1,137,000$ & $1,075,000$ & 2008 & $1,875,000$ \\
\hline 372 & 03234500 & Scioto River at Higby, Ohio & 5,131 & $5,531,000$ & 4 & $1931-2011$ & 81 & $3,490,000$ & $3,542,000$ & 1996 & $5,937,000$ \\
\hline 373 & 03253500 & $\begin{array}{l}\text { Licking River at Catawba, } \\
\quad \text { Kentucky }\end{array}$ & 3,300 & $4,148,000$ & 12 & $\begin{array}{c}1916-17 \\
1929 \\
2011\end{array}$ & 85 & $2,954,000$ & $3,015,000$ & 1979 & $5,596,000$ \\
\hline 376 & 03274000 & $\begin{array}{l}\text { Great Miami River at Hamil- } \\
\text { ton, Ohio }\end{array}$ & 3,630 & $4,293,000$ & 2 & $1928-2011$ & 84 & $2,534,000$ & $2,546,000$ & 2008 & $4,604,000$ \\
\hline 377 & 03276500 & $\begin{array}{l}\text { Whitewater River at Brookville, } \\
\text { Indiana }\end{array}$ & 1,224 & $1,491,000$ & 9 & $\begin{array}{c}1916-17 \\
1924- \\
2011\end{array}$ & 90 & $1,014,000$ & 991,800 & 1996 & $1,738,000$ \\
\hline 379 & 03282000 & $\begin{array}{l}\text { Kentucky River at Lock } 14 \text { at } \\
\text { Heidelberg, Kentucky }\end{array}$ & 2,657 & $3,222,000$ & 21 & $\begin{array}{c}1926-31 \\
1939 \\
2011\end{array}$ & 79 & $2,686,000$ & $2,692,000$ & 1994 & $5,046,000$ \\
\hline 380 & 03284500 & $\begin{array}{l}\text { Kentucky River at Lock } 8 \text { near } \\
\text { Camp Nelson, Kentucky }\end{array}$ & 4,414 & $5,061,000$ & 10 & $\begin{array}{l}1940-71 \\
2003-11\end{array}$ & 41 & $4,134,000$ & $4,087,000$ & 2004 & $6,574,000$ \\
\hline
\end{tabular}


Expected annual runoff in acre-feet for selected annual exceedance probabilities (AEP) based on Water Resources Council Bulletin 17B methods

\section{Estimated \\ AEP for \\ observed \\ 2011 \\ annual \\ runoff (percent)}

\begin{tabular}{cc}
\hline $\begin{array}{c}\text { 2-percent AEP } \\
\text { (50-year recurrence) }\end{array}$ \\
\cline { 2 - 2 } Estimate
\end{tabular}

Estimate
Low
High

1-percent AEP

(100-year recurrence)

95-percent confidence limit

Estimate
Low
High 0.2-percent AEP

(500-year recurrence)

95-percent confidence limit

Estimate
Low
High

\begin{tabular}{|c|c|c|c|c|c|c|c|c|c|}
\hline \multicolumn{10}{|c|}{ Ohio River Basin-Continued } \\
\hline 4 to 10 & $5,965,000$ & $5,420,000$ & $6,838,000$ & $6,290,000$ & $5,578,000$ & $7,380,000$ & $6,950,000$ & $5,781,000$ & $8,655,000$ \\
\hline$>10$ & $8,890,000$ & $7,067,000$ & $9,901,000$ & $9,207,000$ & $6,856,000$ & $10,500,000$ & $9,749,000$ & $6,778,000$ & $11,890,000$ \\
\hline$>10$ & $2,610,000$ & $2,343,000$ & $3,057,000$ & $2,770,000$ & $2,422,000$ & $3,334,000$ & $3,102,000$ & $2,528,000$ & $3,999,000$ \\
\hline 4 to 10 & $1,214,000$ & $1,018,000$ & $1,354,000$ & $1,265,000$ & $1,000,000$ & $1,443,000$ & $1,354,000$ & 983,800 & $1,647,000$ \\
\hline$>10$ & $5,641,000$ & $5,163,000$ & $6,352,000$ & $5,928,000$ & $5,299,000$ & $6,813,000$ & $6,504,000$ & $5,473,000$ & $7,882,000$ \\
\hline$>10$ & $2,653,000$ & $2,419,000$ & $3,117,000$ & $2,820,000$ & $2,527,000$ & $3,420,000$ & $3,178,000$ & $2,718,000$ & $4,161,000$ \\
\hline$>10$ & $2,902,000$ & $2,700,000$ & $3,207,000$ & $3,023,000$ & $2,763,000$ & $3,400,000$ & $3,266,000$ & $2,845,000$ & $3,839,000$ \\
\hline$>10$ & $14,220,000$ & $13,250,000$ & $15,840,000$ & $15,090,000$ & $13,880,000$ & $17,180,000$ & $16,960,000$ & $15,070,000$ & $20,390,000$ \\
\hline$>10$ & $2,276,000$ & $2,102,000$ & $2,570,000$ & $2,393,000$ & $2,171,000$ & $2,762,000$ & $2,634,000$ & $2,279,000$ & $3,210,000$ \\
\hline$>10$ & $16,590,000$ & $15,250,000$ & $18,760,000$ & $17,370,000$ & $15,640,000$ & $20,060,000$ & $18,950,000$ & $16,140,000$ & $23,090,000$ \\
\hline$>10$ & $2,947,000$ & $2,295,000$ & $3,315,000$ & $3,064,000$ & $2,225,000$ & $3,538,000$ & $3,264,000$ & $2,202,000$ & $4,058,000$ \\
\hline$>10$ & $1,322,000$ & 912,800 & $1,541,000$ & $1,376,000$ & 828,500 & $1,652,000$ & $1,470,000$ & 801,600 & $1,929,000$ \\
\hline$>10$ & $1,686,000$ & 951,400 & $1,988,000$ & $1,746,000$ & 881,900 & $2,128,000$ & $1,848,000$ & 746,300 & $2,499,000$ \\
\hline
\end{tabular}

\begin{tabular}{|c|c|c|c|c|c|c|c|c|c|}
\hline 1 to 2 & $1,804,000$ & $1,206,000$ & $2,048,000$ & $1,863,000$ & $1,147,000$ & $2,189,000$ & $1,955,000$ & $1,241,000$ & $2,523,000$ \\
\hline 4 to 10 & $5,824,000$ & $4,097,000$ & $6,572,000$ & $6,034,000$ & $3,995,000$ & $7,010,000$ & $6,376,000$ & $4,107,000$ & $8,041,000$ \\
\hline$>10$ & $4,919,000$ & $3,411,000$ & $5,551,000$ & $5,091,000$ & $3,320,000$ & $5,920,000$ & $5,368,000$ & $3,458,000$ & $6,790,000$ \\
\hline 2 to 4 & $4,466,000$ & $3,155,000$ & $5,113,000$ & $4,657,000$ & $3,052,000$ & $5,505,000$ & $4,975,000$ & $3,087,000$ & $6,432,000$ \\
\hline$>10$ & $1,744,000$ & $1,136,000$ & $2,003,000$ & $1,810,000$ & $1,087,000$ & $2,155,000$ & $1,915,000$ & $1,167,000$ & $2,518,000$ \\
\hline$>10$ & $4,749,000$ & $4,139,000$ & $5,508,000$ & $5,034,000$ & $4,192,000$ & $5,989,000$ & $5,591,000$ & $4,198,000$ & $7,134,000$ \\
\hline$>10$ & $6,719,000$ & $4,140,000$ & $7,887,000$ & $6,989,000$ & $3,861,000$ & $8,467,000$ & $7,456,000$ & $3,697,000$ & $9,942,000$ \\
\hline
\end{tabular}


Table 2. Annual runoff data and results of flood-probability analyses for selected streamgages in the Central United States affected by 2011 flooding.-Continued

[Stations used in the trend analysis are italicized. $\mathrm{mi}^{2}$, square miles; AEP, annual exceedance probability; $<$, less than; $>$, greater than]

\begin{tabular}{|c|c|c|c|c|c|c|c|c|c|c|c|}
\hline \multirow{2}{*}{ 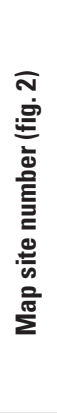 } & \multirow[b]{2}{*}{$\begin{array}{l}\text { Station } \\
\text { number }\end{array}$} & \multirow[b]{2}{*}{ Station name } & \multirow{2}{*}{ 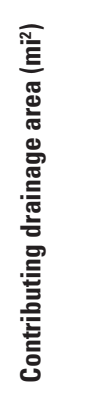 } & \multicolumn{2}{|c|}{ Water year 2011} & \multicolumn{4}{|c|}{ Period of record } & \multicolumn{2}{|c|}{$\begin{array}{l}\text { Previous maximum } \\
\text { annual runoff based } \\
\text { on data through } \\
\text { water year } 2010^{\mathrm{a}}\end{array}$} \\
\hline & & & & 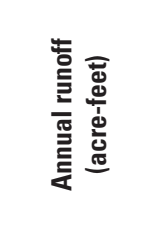 & 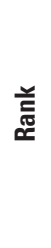 & 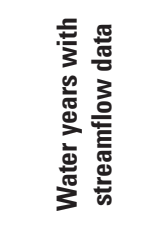 & 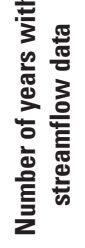 & 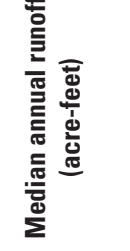 & 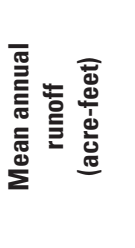 & 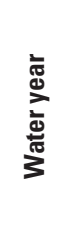 & 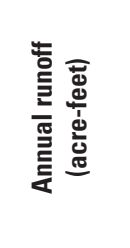 \\
\hline \multicolumn{12}{|c|}{ Ohio River Basin-Continued } \\
\hline 381 & 03290500 & $\begin{array}{c}\text { Kentucky River at Lock } 2 \text { at } \\
\text { Lockport, Kentucky }\end{array}$ & 5,984 & $7,240,000$ & 22 & $\begin{array}{l}1926-30 \\
1933-37 \\
1940- \\
2011\end{array}$ & 82 & $5,919,000$ & $6,050,000$ & 1927 & $10,570,000$ \\
\hline
\end{tabular}

\begin{tabular}{|c|c|c|c|c|c|c|c|c|c|c|c|}
\hline 383 & 03294500 & $\begin{array}{l}\text { Ohio River at Louisville, } \\
\quad \text { Kentucky }\end{array}$ & 91,170 & $110,800,000$ & 9 & 1929-2011 & 83 & $84,710,000$ & $85,500,000$ & 2004 & $133,900,000$ \\
\hline 384 & 03298500 & $\begin{array}{l}\text { Salt River at Shepherdsville, } \\
\quad \text { Kentucky }\end{array}$ & 1,197 & $1,586,000$ & 13 & 1939-2011 & 73 & $1,100,000$ & $1,169,000$ & 1979 & $2,621,000$ \\
\hline 386 & 03308500 & $\begin{array}{l}\text { Green River at Munfordville, } \\
\quad \text { Kentucky }\end{array}$ & 1,493 & $2,389,000$ & 24 & $\begin{array}{l}1916-22 \\
1928-31 \\
1938- \\
2011\end{array}$ & 85 & $1,984,000$ & $1,974,000$ & 1979 & $3,830,000$ \\
\hline 387 & 03320000 & $\begin{array}{l}\text { Green River at Lock } 2 \text { at Cal- } \\
\text { houn, Kentucky }\end{array}$ & 6,032 & $10,500,000$ & 14 & $1931-2011$ & 81 & $8,036,000$ & $8,080,000$ & 1979 & $16,000,000$ \\
\hline 388 & 03325000 & $\begin{array}{l}\text { Wabash River at Wabash, } \\
\text { Indiana }\end{array}$ & 1,768 & $1,651,000$ & 11 & $\begin{array}{c}1924-34 \\
1936- \\
2011\end{array}$ & 87 & $1,187,000$ & $1,165,000$ & 1950 & $2,165,000$ \\
\hline 389 & 03329000 & $\begin{array}{l}\text { Wabash River at Logansport, } \\
\text { Indiana }\end{array}$ & 3,779 & $3,316,000$ & 20 & $1924-2011$ & 88 & $2,530,000$ & $2,564,000$ & 1950 & $4,785,000$ \\
\hline 390 & 03336000 & $\begin{array}{l}\text { Wabash River at Covington, } \\
\text { Indiana }\end{array}$ & 8,218 & $7,131,000$ & 18 & $1940-2011$ & 72 & $5,596,000$ & $5,729,000$ & 1950 & $10,860,000$ \\
\hline 391 & 03341500 & $\begin{array}{l}\text { Wabash River at Terre Haute, } \\
\text { Indiana }\end{array}$ & 12,263 & $10,430,000$ & 16 & $1928-2011$ & 84 & $8,434,000$ & $8,311,000$ & 1950 & $16,510,000$ \\
\hline 392 & 03351000 & $\begin{array}{l}\text { White River near Nora, } \\
\quad \text { Indiana }\end{array}$ & 1,219 & $1,224,000$ & 14 & $1930-2011$ & 82 & 883,300 & 869,300 & 2007 & $1,491,000$ \\
\hline 393 & 03360500 & $\begin{array}{l}\text { White River at Newberry, } \\
\text { Indiana }\end{array}$ & 4,688 & $4,858,000$ & 15 & 1929-2011 & 83 & $3,757,000$ & $3,688,000$ & 2008 & $6,458,000$ \\
\hline 394 & 03373500 & $\begin{array}{l}\text { East Fork White River at } \\
\text { Shoals, Indiana }\end{array}$ & 4,927 & $6,154,000$ & 9 & $\begin{array}{l}\text { 1904-05, } \\
\quad 1910-16 \\
1924- \\
2011\end{array}$ & 97 & $4,358,000$ & $4,181,000$ & 1950 & $7,529,000$ \\
\hline 395 & 03374000 & $\begin{array}{l}\text { White River at Petersburg, } \\
\text { Indiana }\end{array}$ & 11,125 & $13,030,000$ & 7 & 1929-2011 & 83 & $9,629,000$ & $9,171,000$ & 1950 & $16,510,000$ \\
\hline 398 & 03376500 & $\begin{array}{l}\text { Patoka River near Princeton, } \\
\quad \text { Indiana }\end{array}$ & 822 & $1,173,000$ & 11 & $1935-2011$ & 77 & 760,200 & 772,200 & 1950 & $1,506,000$ \\
\hline 399 & 03377500 & $\begin{array}{l}\text { Wabash River at Mount Car- } \\
\quad \text { mel, Illinois }\end{array}$ & 28,635 & $29,320,000$ & 11 & $1928-2011$ & 84 & $22,370,000$ & $21,240,000$ & 1950 & $41,050,000$ \\
\hline 402 & 03381500 & $\begin{array}{l}\text { Little Wabash River at Carmi, } \\
\quad \text { Illinois }\end{array}$ & 3,102 & $3,685,000$ & 3 & $1940-2011$ & 72 & $2,255,000$ & $2,081,000$ & 1950 & $4,409,000$ \\
\hline 407 & 03404500 & $\begin{array}{l}\text { Cumberland River at Cumber- } \\
\text { land Falls, Kentucky }\end{array}$ & 1,977 & $3,598,000$ & 4 & $\begin{array}{l}\text { 1908-11, } \\
\text { 1916-31, } \\
1933-94 \\
2003-05 \\
2011\end{array}$ & 86 & $2,349,000$ & $2,345,000$ & 1927 & $3,765,000$ \\
\hline
\end{tabular}




\begin{tabular}{|c|c|c|c|c|c|c|c|c|c|}
\hline \multirow{3}{*}{$\begin{array}{c}\text { Estimated } \\
\text { AEP for } \\
\text { observed } \\
2011 \\
\text { annual } \\
\text { runoff } \\
\text { (percent) }\end{array}$} & \multicolumn{3}{|c|}{$\begin{array}{c}\text { 2-percent AEP } \\
\text { (50-year recurrence) }\end{array}$} & \multicolumn{3}{|c|}{$\begin{array}{c}\text { 1-percent AEP } \\
\text { (100-year recurrence) }\end{array}$} & \multicolumn{3}{|c|}{$\begin{array}{c}\text { 0.2-percent AEP } \\
\text { (500-year recurrence) }\end{array}$} \\
\hline & \multirow[b]{2}{*}{ Estimate } & \multicolumn{2}{|c|}{ 95-percent confidence limit } & \multirow[b]{2}{*}{ Estimate } & \multicolumn{2}{|c|}{ 95-percent confidence limit } & \multirow[b]{2}{*}{ Estimate } & \multicolumn{2}{|c|}{ 95-percent confidence limit } \\
\hline & & Low & High & & Low & High & & Low & High \\
\hline \multicolumn{10}{|c|}{ Ohio River Basin-Continued } \\
\hline$>10$ & $10,280,000$ & $8,786,000$ & $11,700,000$ & $10,800,000$ & $8,719,000$ & $12,590,000$ & $11,760,000$ & $8,541,000$ & $14,660,000$ \\
\hline$>10$ & $126,200,000$ & $114,700,000$ & $138,600,000$ & $131,000,000$ & $115,200,000$ & $146,300,000$ & $140,000,000$ & $114,800,000$ & $163,700,000$ \\
\hline$>10$ & $2,037,000$ & $1,425,000$ & $2,365,000$ & $2,131,000$ & $1,349,000$ & $2,556,000$ & $2,292,000$ & $1,336,000$ & $3,020,000$ \\
\hline$>10$ & $3,459,000$ & $2,976,000$ & $3,964,000$ & $3,651,000$ & $2,980,000$ & $4,290,000$ & $4,016,000$ & $2,944,000$ & $5,055,000$ \\
\hline$>10$ & $14,040,000$ & $11,950,000$ & $16,100,000$ & $14,790,000$ & $11,880,000$ & $17,390,000$ & $16,200,000$ & $11,630,000$ & $20,430,000$ \\
\hline$>10$ & $1,924,000$ & $1,135,000$ & $2,242,000$ & $1,967,000$ & $1,066,000$ & $2,404,000$ & $2,022,000$ & $1,293,000$ & $2,823,000$ \\
\hline$>10$ & $4,132,000$ & $2,670,000$ & $4,702,000$ & $4,235,000$ & $2,529,000$ & $5,006,000$ & $4,380,000$ & $2,851,000$ & $5,750,000$ \\
\hline$>10$ & $9,126,000$ & $5,997,000$ & $10,330,000$ & $9,386,000$ & $5,691,000$ & $10,990,000$ & $9,783,000$ & $6,040,000$ & $12,600,000$ \\
\hline$>10$ & $13,820,000$ & $9,066,000$ & $15,740,000$ & $14,260,000$ & $8,597,000$ & $16,820,000$ & $14,930,000$ & $9,302,000$ & $19,410,000$ \\
\hline$>10$ & $1,509,000$ & 925,200 & $1,749,000$ & $1,560,000$ & 870,100 & $1,882,000$ & $1,635,000$ & 959,500 & $2,210,000$ \\
\hline$>10$ & $6,029,000$ & $3,592,000$ & $6,996,000$ & $6,165,000$ & $3,376,000$ & $7,489,000$ & $6,345,000$ & $4,032,000$ & $8,768,000$ \\
\hline$>10$ & $6,862,000$ & $3,896,000$ & $8,092,000$ & $6,990,000$ & $3,703,000$ & $8,693,000$ & $7,148,000$ & $4,673,000$ & $10,320,000$ \\
\hline$>10$ & $14,700,000$ & $8,457,000$ & $17,230,000$ & $14,960,000$ & $7,946,000$ & $18,460,000$ & $15,280,000$ & $10,090,000$ & $21,830,000$ \\
\hline 4 to 10 & $1,304,000$ & 726,100 & $1,538,000$ & $1,336,000$ & 677,600 & $1,658,000$ & $1,378,000$ & 823,700 & $1,978,000$ \\
\hline$>10$ & $33,810,000$ & $20,000,000$ & $39,330,000$ & $34,430,000$ & $18,820,000$ & $42,040,000$ & $35,210,000$ & $23,380,000$ & $49,280,000$ \\
\hline 1 to 2 & $3,635,000$ & $1,458,000$ & $4,749,000$ & $3,690,000$ & $1,609,000$ & $5,303,000$ & $3,747,000$ & $2,241,000$ & $7,186,000$ \\
\hline 2 to 4 & $3,719,000$ & $3,241,000$ & $4,141,000$ & $3,877,000$ & $3,215,000$ & $4,405,000$ & $4,163,000$ & $3,160,000$ & $5,011,000$ \\
\hline
\end{tabular}


Table 2. Annual runoff data and results of flood-probability analyses for selected streamgages in the Central United States affected by 2011 flooding.-Continued

[Stations used in the trend analysis are italicized. $\mathrm{mi}^{2}$, square miles; AEP, annual exceedance probability; <, less than; >, greater than]

\begin{tabular}{|c|c|c|c|c|c|c|c|c|c|c|c|}
\hline \multirow{2}{*}{ 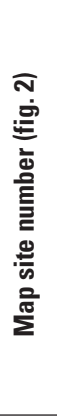 } & \multirow[b]{2}{*}{$\begin{array}{l}\text { Station } \\
\text { number }\end{array}$} & \multirow[b]{2}{*}{ Station name } & \multirow{2}{*}{ 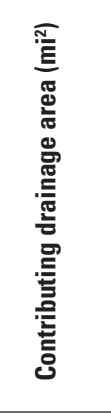 } & \multicolumn{2}{|c|}{ Water year 2011} & \multicolumn{4}{|c|}{ Period of record } & \multicolumn{2}{|c|}{$\begin{array}{c}\text { Previous maximum } \\
\text { annual runoff based } \\
\text { on data through } \\
\text { water year } 2010^{\mathrm{a}}\end{array}$} \\
\hline & & & & 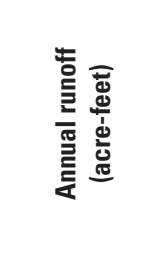 & 恙 & 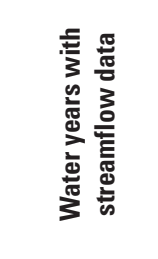 & 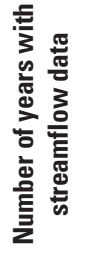 & 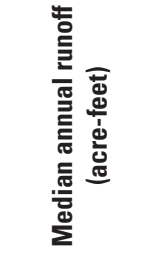 & 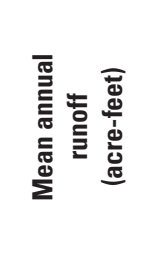 & 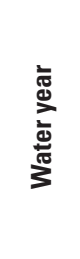 & 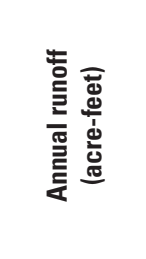 \\
\hline \multicolumn{12}{|c|}{ Ohio River Basin-Continued } \\
\hline 408 & 03431500 & $\begin{array}{l}\text { Cumberland River at Nash- } \\
\text { ville, Tennessee }\end{array}$ & 12,856 & $16,650,000$ & 27 & $\begin{array}{l}1893-1954 \\
1993- \\
2011\end{array}$ & 81 & $14,330,000$ & $14,700,000$ & 1920 & $25,340,000$ \\
\hline 409 & 03455000 & $\begin{array}{l}\text { French Broad River near } \\
\text { Newport, Tennessee }\end{array}$ & 1,858 & $1,940,000$ & 55 & $\begin{array}{l}\text { 1904-05 } \\
1921-94 \\
1997- \\
2011\end{array}$ & 91 & $2,114,000$ & $2,111,000$ & 1973 & $3,359,000$ \\
\hline 410 & 03528000 & $\begin{array}{l}\text { Clinch River Above Tazewell, } \\
\text { Tennessee }\end{array}$ & 1,474 & $1,614,000$ & 35 & $1920-2011$ & 92 & $1,528,000$ & $1,479,000$ & 1927 & $2,367,000$ \\
\hline 411 & 03532000 & $\begin{array}{l}\text { Powell River near Arthur, } \\
\text { Tennessee }\end{array}$ & 685 & 905,000 & 24 & $\begin{array}{l}\text { 1920-1981, } \\
1997- \\
2011\end{array}$ & 77 & 818,100 & 803,600 & 1974 & $1,347,000$ \\
\hline 412 & 03566000 & $\begin{array}{l}\text { Hiwassee River at Charleston, } \\
\text { Tennessee }\end{array}$ & 2,298 & $2,317,000$ & 51 & $\begin{array}{l}\text { 1901-02, } \\
1921-39, \\
1964-76, \\
1980-81, \\
1988- \\
2011\end{array}$ & 61 & $3,410,000$ & $3,319,000$ & 1990 & $4,988,000$ \\
\hline 413 & 03584600 & $\begin{array}{l}\text { Elk River at Prospect, Ten- } \\
\text { nessee }\end{array}$ & 1,805 & $2,056,000$ & 57 & $\begin{array}{l}\text { 1905-07, } \\
1920-94, \\
1999- \\
2001 \\
2004- \\
2011\end{array}$ & 89 & $2,194,000$ & $2,198,000$ & 1920 & $3,946,000$ \\
\hline 414 & 03603000 & $\begin{array}{l}\text { Duck River above Hurricane } \\
\text { Mills, Tennessee }\end{array}$ & 2,557 & $2,700,000$ & 44 & $\begin{array}{l}\text { 1926-94, } \\
\text { 1999- } \\
2001 \\
2009-11\end{array}$ & 75 & $2,860,000$ & $2,966,000$ & 1973 & $6,313,000$ \\
\hline 416 & 03611500 & $\begin{array}{l}\text { Ohio River at Metropolis, } \\
\text { Illinois }\end{array}$ & 203,000 & $233,100,000$ & 26 & $1929-2011$ & 83 & $201,300,000$ & $202,100,000$ & 1979 & $316,400,000$ \\
\hline \multicolumn{12}{|c|}{ Lower Mississippi River Basin (downstream from the mouth of the Ohio River) } \\
\hline 420 & 07026040 & $\begin{array}{l}\text { Obion River at Highway } 51 \\
\text { near Obion, Tennessee }\end{array}$ & 1,875 & $2,201,000$ & 24 & $\begin{array}{l}1930-58 \\
1967-95 \\
2002-11\end{array}$ & 68 & $1,940,000$ & $1,995,000$ & 1973 & $3,873,000$ \\
\hline 421 & 07030050 & $\begin{array}{l}\text { Hatchie River at Rialto, Ten- } \\
\text { nessee }\end{array}$ & 2,308 & $2,252,000$ & 24 & $\begin{array}{c}1941-74, \\
1977-78, \\
1980-84, \\
1986-88, \\
2004-11\end{array}$ & 52 & $2,186,000$ & $2,333,000$ & 1973 & $4,894,000$ \\
\hline 426 & 07032000 & $\begin{array}{l}\text { Mississippi River at Memphis, } \\
\text { Tennessee }\end{array}$ & 932,800 & $515,500,000$ & 4 & 1934-2011 & 78 & $374,100,000$ & $365,800,000$ & 1973 & $571,100,000$ \\
\hline 437 & 07047970 & $\begin{array}{l}\text { Mississippi River at Helena, } \\
\text { Arkansas }\end{array}$ & 941,800 & $511,100,000$ & 7 & 1929-2011 & 83 & $378,900,000$ & $369,700,000$ & 1973 & $589,400,000$ \\
\hline 443 & 07060500 & $\begin{array}{l}\text { White River at Calico Rock, } \\
\text { Arkansas }\end{array}$ & 9,980 & $9,701,000$ & 18 & $1940-2011$ & 72 & $7,370,000$ & $7,430,000$ & 1945 & $16,580,000$ \\
\hline 444 & 07061000 & $\begin{array}{l}\text { White River at Batesville, } \\
\text { Arkansas }\end{array}$ & 11,070 & $11,290,000$ & 10 & $\begin{array}{l}1938-58 \\
1987-94 \\
2001-11\end{array}$ & 40 & $9,593,000$ & $9,157,000$ & 1945 & $19,190,000$ \\
\hline
\end{tabular}




\begin{tabular}{|c|c|c|c|c|c|c|c|c|c|}
\hline \multirow{4}{*}{$\begin{array}{l}\text { Estimated } \\
\text { AEP for } \\
\text { observed } \\
2011 \\
\text { annual } \\
\text { runoff } \\
\text { (percent) }\end{array}$} & \multicolumn{9}{|c|}{ Expected annual runoff in acre-feet for selected annual exceedance probabilities (AEP) based on Water Resources Council Bulletin 17B methods } \\
\hline & \multicolumn{3}{|c|}{$\begin{array}{c}\text { 2-percent AEP } \\
\text { (50-year recurrence) }\end{array}$} & \multicolumn{3}{|c|}{$\begin{array}{c}\text { 1-percent AEP } \\
\text { (100-year recurrence) }\end{array}$} & \multicolumn{3}{|c|}{$\begin{array}{c}\text { 0.2-percent AEP } \\
\text { (500-year recurrence) }\end{array}$} \\
\hline & \multirow[b]{2}{*}{ Estimate } & \multicolumn{2}{|c|}{ 95-percent confidence limit } & \multirow[b]{2}{*}{ Estimate } & \multicolumn{2}{|c|}{ 95-percent confidence limit } & \multirow[b]{2}{*}{ Estimate } & \multicolumn{2}{|c|}{ 95-percent confidence limit } \\
\hline & & Low & High & & Low & High & & Low & High \\
\hline \multicolumn{10}{|c|}{ Ohio River Basin-Continued } \\
\hline$>10$ & $25,190,000$ & $22,670,000$ & $29,610,000$ & $26,870,000$ & $23,630,000$ & $32,520,000$ & $30,410,000$ & $25,120,000$ & $39,540,000$ \\
\hline$>10$ & $3,334,000$ & $3,031,000$ & $3,724,000$ & $3,496,000$ & $3,088,000$ & $3,980,000$ & $3,812,000$ & $3,141,000$ & $4,570,000$ \\
\hline$>10$ & $2,333,000$ & $2,076,000$ & $2,588,000$ & $2,434,000$ & $2,080,000$ & $2,753,000$ & $2,623,000$ & $2,066,000$ & $3,129,000$ \\
\hline$>10$ & $1,305,000$ & $1,160,000$ & $1,480,000$ & $1,370,000$ & $1,170,000$ & $1,588,000$ & $1,495,000$ & $1,169,000$ & $1,840,000$ \\
\hline$>10$ & $5,214,000$ & $4,168,000$ & $5,886,000$ & $5,413,000$ & $3,994,000$ & $6,259,000$ & $5,762,000$ & $3,853,000$ & $7,144,000$ \\
\hline$>10$ & $3,713,000$ & $3,051,000$ & $4,187,000$ & $3,880,000$ & $2,985,000$ & $4,488,000$ & $4,177,000$ & $2,922,000$ & $5,182,000$ \\
\hline
\end{tabular}

$\begin{array}{rrrrrrrrrr}>10 & 5,215,000 & 4,555,000 & 6,079,000 & 5,531,000 & 4,624,000 & 6,618,000 & 6,155,000 & 4,645,000 & 7,905,000 \\ & & & & & & & & & \\ >10 & 303,100,000 & 265,000,000 & 333,000,000 & 313,800,000 & 261,500,000 & 351,300,000 & 333,000,000 & 256,600,000 & 392,600,000\end{array}$

\begin{tabular}{|c|c|c|c|c|c|c|c|c|c|}
\hline \multicolumn{10}{|c|}{ Lower Mississippi River Basin (downstream from the mouth of the Ohio River) } \\
\hline$>10$ & $3,552,000$ & $2,303,000$ & $4,137,000$ & $3,711,000$ & $2,188,000$ & $4,475,000$ & $3,979,000$ & $2,235,000$ & $5,302,000$ \\
\hline$>10$ & $4,516,000$ & $3,842,000$ & $5,992,000$ & $4,910,000$ & $4,034,000$ & $6,833,000$ & $5,765,000$ & $4,303,000$ & $9,030,000$ \\
\hline
\end{tabular}

\begin{tabular}{rrrrrrrrrrr}
\hline 4 to 10 & $541,500,000$ & $489,000,000$ & $598,200,000$ & $562,500,000$ & $490,100,000$ & $632,500,000$ & $601,900,000$ & $486,000,000$ & $710,500,000$ \\
\hline 4 to 10 & $546,500,000$ & $486,800,000$ & $600,000,000$ & $566,200,000$ & $483,800,000$ & $632,600,000$ & $602,200,000$ & $476,300,000$ & $706,600,000$ \\
\hline 10 & $15,510,000$ & $13,280,000$ & $19,850,000$ & $17,020,000$ & $14,090,000$ & $22,750,000$ & $20,320,000$ & $15,350,000$ & $30,280,000$ \\
\hline 10 & $17,480,000$ & $13,910,000$ & $22,380,000$ & $18,680,000$ & $13,620,000$ & $24,830,000$ & $21,060,000$ & $12,650,000$ & $31,170,000$
\end{tabular}




\section{Annual Exceedance Probabilities and Trends for the Central United States during the 2011 Floods}

Table 2. Annual runoff data and results of flood-probability analyses for selected streamgages in the Central United States affected by 2011 flooding.-Continued

[Stations used in the trend analysis are italicized. $\mathrm{mi}^{2}$, square miles; AEP, annual exceedance probability; <, less than; $>$, greater than]

\begin{tabular}{|c|c|c|c|c|c|c|c|c|c|c|c|}
\hline \multirow{2}{*}{ 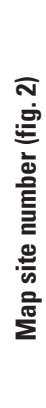 } & \multirow[b]{2}{*}{$\begin{array}{l}\text { Station } \\
\text { number }\end{array}$} & \multirow[b]{2}{*}{ Station name } & \multirow{2}{*}{ 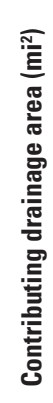 } & \multicolumn{2}{|c|}{ Water year 2011} & \multicolumn{4}{|c|}{ Period of record } & \multicolumn{2}{|c|}{$\begin{array}{c}\text { Previous maximum } \\
\text { annual runoff based } \\
\text { on data through } \\
\text { water year } 2010^{\mathrm{a}}\end{array}$} \\
\hline & & & & 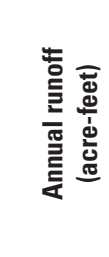 & 兰 & 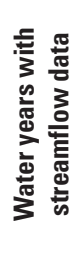 & 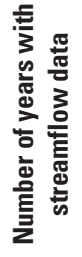 & 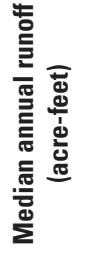 & 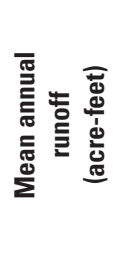 & 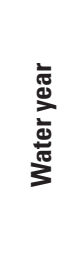 & 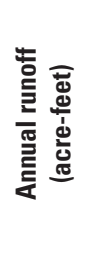 \\
\hline
\end{tabular}

\begin{tabular}{|c|c|c|c|c|c|c|c|c|c|c|c|}
\hline \multicolumn{12}{|c|}{ Lower Mississippi River Basin (downstream from the mouth of the Ohio River)_Continued } \\
\hline 447 & 07064000 & $\begin{array}{l}\text { Black River near Corning, } \\
\text { Arkansas }\end{array}$ & 1,750 & $1,998,000$ & 11 & $\begin{array}{c}\text { 1939-95, } \\
1999- \\
2011\end{array}$ & 70 & $1,321,000$ & $1,368,000$ & 1973 & $2,903,000$ \\
\hline 448 & 07068000 & $\begin{array}{l}\text { Current River at Doniphan, } \\
\text { Missouri }\end{array}$ & 2,038 & $2,773,000$ & 11 & $1922-2011$ & 90 & $1,991,000$ & $2,035,000$ & 1985 & $4,243,000$ \\
\hline 451 & 07072500 & $\begin{array}{l}\text { Black River at Black Rock, } \\
\text { Arkansas }\end{array}$ & 7,370 & $8,688,000$ & 13 & $\begin{array}{c}1930-31 \\
1940- \\
2011\end{array}$ & 74 & $5,814,000$ & $6,321,000$ & 1973 & $12,520,000$ \\
\hline 455 & 07077000 & $\begin{array}{l}\text { White River at DeValls Bluff, } \\
\text { Arkansas }\end{array}$ & 23,400 & $21,860,000$ & 20 & $\begin{array}{c}1950-1970 \\
1989- \\
2011\end{array}$ & 44 & $19,870,000$ & $19,460,000$ & 1950 & $37,140,000$ \\
\hline 461 & 07144300 & $\begin{array}{l}\text { Arkansas River at Wichita, } \\
\quad \text { Kansas }\end{array}$ & 33,227 & 154,200 & 75 & 1935-2011 & 77 & 631,300 & 754,900 & 1993 & $2,787,000$ \\
\hline 462 & 07151000 & $\begin{array}{l}\text { Salt Fork Arkansas River at } \\
\text { Tonkawa, Oklahoma }\end{array}$ & 4,470 & 106,400 & 72 & 1936-2011 & 76 & 568,700 & 650,800 & 1999 & $2,693,000$ \\
\hline 463 & 07152000 & $\begin{array}{l}\text { Chikaskia River near Black- } \\
\text { well, Oklahoma }\end{array}$ & 1,873 & 73,850 & 71 & $1937-2011$ & 75 & 362,000 & 449,900 & 1999 & $1,441,000$ \\
\hline 464 & 07152500 & $\begin{array}{l}\text { Arkansas River at Ralston, } \\
\text { Oklahoma }\end{array}$ & 46,631 & 810,900 & 84 & 1926-2011 & 86 & $3,584,000$ & $3,965,000$ & 1999 & $12,160,000$ \\
\hline 465 & 07160000 & $\begin{array}{l}\text { Cimarron River near Guthrie, } \\
\text { Oklahoma }\end{array}$ & 12,932 & 160,700 & 66 & $\begin{array}{c}1938-76 \\
1984- \\
2011\end{array}$ & 67 & 680,500 & 831,100 & 1987 & $2,824,000$ \\
\hline 466 & 07164500 & $\begin{array}{l}\text { Arkansas River at Tulsa, } \\
\text { Oklahoma }\end{array}$ & 62,811 & 688,500 & 86 & $1926-2011$ & 86 & $5,350,000$ & $5,610,000$ & 1999 & $16,580,000$ \\
\hline 467 & 07176000 & $\begin{array}{l}\text { Verdigris River near Clare- } \\
\text { more, Oklahoma }\end{array}$ & 6,451 & 883,300 & 62 & 1936-2011 & 76 & $2,827,000$ & $3,086,000$ & 1999 & $7,891,000$ \\
\hline 468 & 07183000 & $\begin{array}{l}\text { Neosho River near Iola, } \\
\quad \text { Kansas }\end{array}$ & 3,723 & 522,700 & 79 & $\begin{array}{c}1896-97 \\
1899- \\
1903 \\
1918- \\
2011\end{array}$ & 101 & $1,158,000$ & $1,365,000$ & 1951 & $4,807,000$ \\
\hline 475 & 07191500 & $\begin{array}{l}\text { Neosho River near Chouteau, } \\
\text { Oklahoma }\end{array}$ & 11,580 & $4,959,000$ & 42 & $\begin{array}{c}1938-1950 \\
1964- \\
2011\end{array}$ & 61 & $6,081,000$ & $6,517,000$ & 1993 & $16,070,000$ \\
\hline 486 & 07245000 & $\begin{array}{l}\text { Canadian River near White- } \\
\text { field, Oklahoma }\end{array}$ & 39,149 & $1,643,000$ & 60 & 1939-2011 & 73 & $3,707,000$ & $4,317,000$ & 1993 & $11,000,000$ \\
\hline 490 & 07289000 & $\begin{array}{l}\text { Mississippi River at Vicksburg, } \\
\text { Mississippi }\end{array}$ & $1,140,500$ & $568,300,000$ & 10 & 1932-2011 & 80 & $448,100,000$ & $443,400,000$ & 1973 & $720,400,000$ \\
\hline 491 & 07290000 & $\begin{array}{l}\text { Big Black River near Bovina, } \\
\text { Mississippi }\end{array}$ & 2,812 & $1,419,000$ & 68 & 1937-2011 & 75 & $2,592,000$ & $2,766,000$ & 1983 & $7,891,000$ \\
\hline
\end{tabular}

${ }^{a}$ The U.S. Geological Survey water year begins October 1 of the previous calendar year and ends September 30 of the calendar year. 


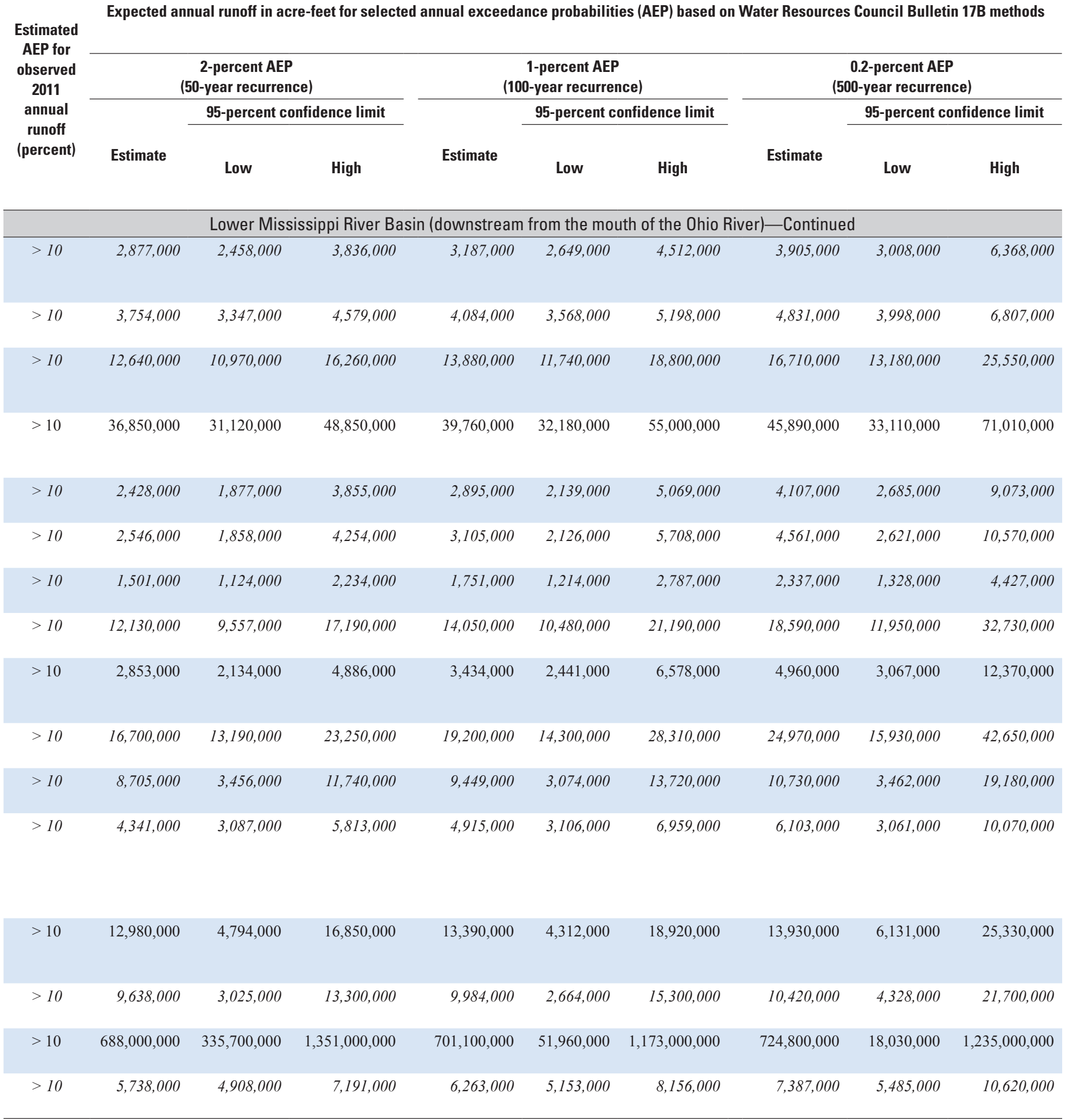


Publishing support provided by:

Rolla Publishing Service Center

For more information concerning this publication, contact:

Chief, USGS Office of Surface Water

415 National Center

12201 Sunrise Valley Drive

Reston, VA 20192

(703) 648-5301

Or visit the Office of Surface Water Web site at: http://water.usgs.gov/osw/ 



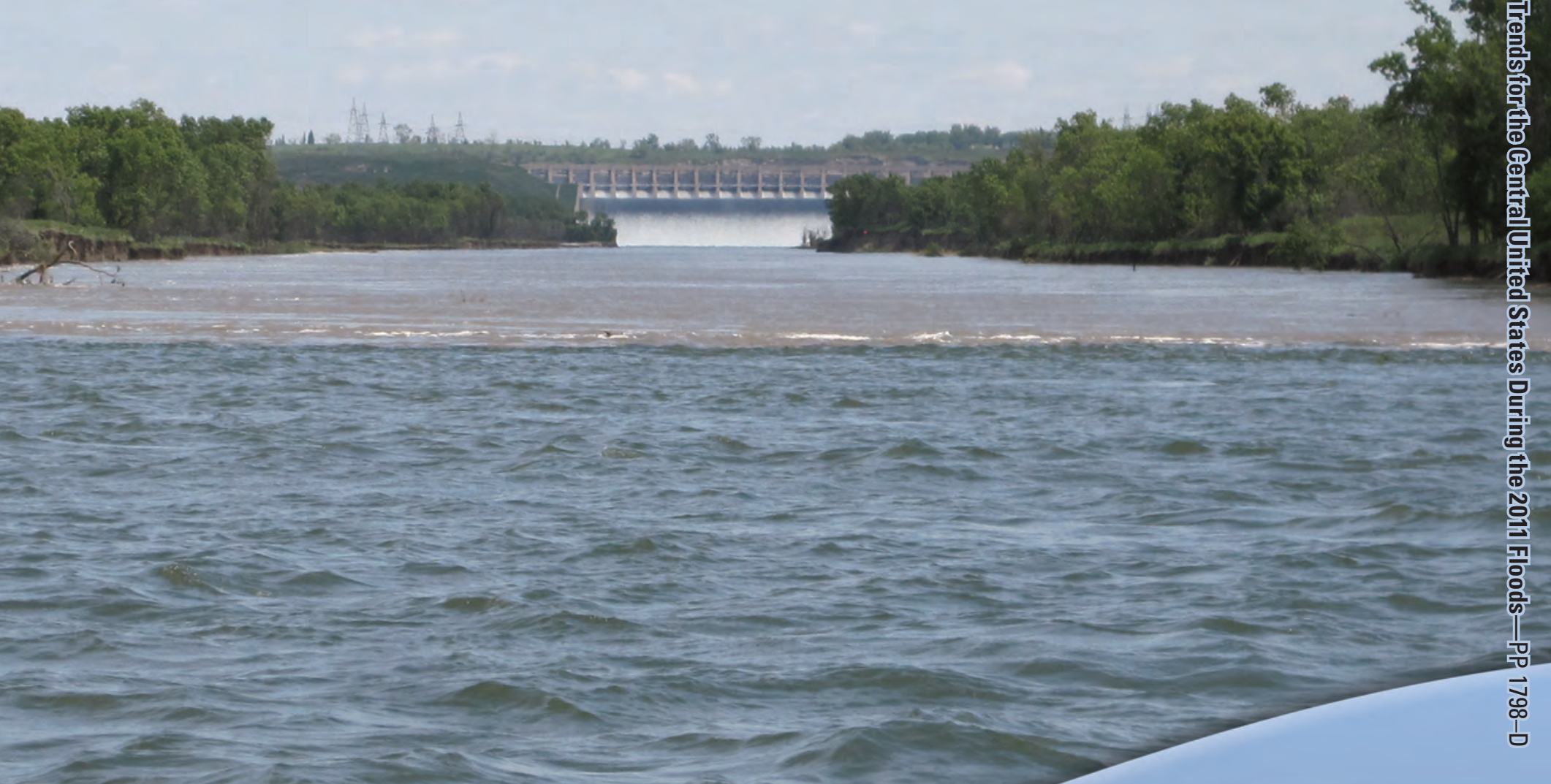

